\title{
Review
}

\section{Supported Metal Catalysts for the Synthesis of $N$-Heterocycles}

\author{
Laura Antonella Aronica ${ }^{1,2} \mathbb{D}$ and Gianluigi Albano ${ }^{3, * \mathbb{D}}$ \\ 1 Dipartimento di Chimica e Chimica Industriale, Università di Pisa, Via Giuseppe Moruzzi 13, \\ 56124 Pisa, Italy; laura.antonella.aronica@unipi.it \\ 2 CIRCC-Consorzio Interuniversitario per le Reattività Chimiche e la Catalisi, Via Celso Ulpiani 27, \\ 70126 Bari, Italy \\ 3 Dipartimento di Chimica, Università degli Studi di Bari "Aldo Moro", Via Edoardo Orabona 4, \\ 70126 Bari, Italy \\ * Correspondence: gianluigi.albano@uniba.it
}

Citation: Aronica, L.A.; Albano, G. Supported Metal Catalysts for the Synthesis of $\mathrm{N}$-Heterocycles. Catalysts 2022, 12, 68. https:// doi.org/10.3390/catal12010068

Academic Editors: Carlos Vila and Ioannis D. Kostas

Received: 29 November 2021

Accepted: 5 January 2022

Published: 9 January 2022

Publisher's Note: MDPI stays neutral with regard to jurisdictional claims in published maps and institutional affiliations.

Copyright: (C) 2022 by the authors. Licensee MDPI, Basel, Switzerland. This article is an open access article distributed under the terms and conditions of the Creative Commons Attribution (CC BY) license (https:// creativecommons.org/licenses/by/ $4.0 /)$.

\begin{abstract}
Nitrogen-containing heterocycles are important scaffolds for a large number of compounds with biological, pharmaceutical, industrial and optoelectronic applications. A wide range of different methodologies for the preparation of $N$-heterocycles are based on metal-catalyzed cyclization of suitable substrates. Due to the growing interest in Green Chemistry criteria over the past two decades, the use of supported metal catalysts in the preparation of $N$-heterocycles has become a central topic in Organic Chemistry. Here we will give a critical overview of all the solid supported metal catalysts applied in the synthesis of $N$-heterocycles, following a systematic approach as a function of the type of support: (i) metal catalysts supported on inorganic matrices; (ii) metal catalysts supported on organic matrices; (iii) metal catalysts supported on hybrid inorganic-organic matrices. In particular, we will try to emphasize the effective heterogeneity and recyclability of the described metal catalysts, specifying which studies were carried out in order to evaluate these aspects.
\end{abstract}

Keywords: cyclizations; heterocycles; metal catalysts; nitrogen heterocycles; supported catalysts

\section{Introduction}

Nitrogen-containing heterocycles represent important scaffolds for a large number of natural products [1,2], biologically active compounds with pharmaceutical interest [3,4], materials for chemical industry (e.g., pigments in paints, varnishes and plastics) [5] and organic optoelectronics (e.g., polymeric active layers for electroluminescent devices or organic photovoltaic cells) [6-8]. This explains the great and continuous interest of the Organic Chemistry community in the development of efficient and convenient methodologies for the preparation of $\mathrm{N}$-heterocycles [9-12].

Although a wide range of different methodologies have been described depending on the structure of the $N$-heterocyclic target, many of them are based on the metal-catalyzed cyclization of suitable substrates. A broad variety of homogeneous catalytic systems, often based on metal complexes bearing suitable ligands, have been successfully developed for the synthesis of $N$-heterocycles [13-16], showing in general high activity and good versatility. However, the growing interest in Green Chemistry criteria [17] over the past two decades has highlighted the main issues of homogeneous metal catalysis: relatively high costs of metal species; low catalyst recoverability and recyclability; metal contamination of final products, often requiring complex purification methods. With environmental regulations becoming increasingly stringent, the need for more sustainable approaches for metal-catalyzed cyclizations became central in the synthesis of $N$-heterocycles.

The use of supported metal catalysts in the preparation of $N$-heterocycles is very attractive: on one hand, these systems can be easily separated from the reaction medium by simple filtration, thus making their recyclability possible; on the other hand, heterogeneous catalysis in some cases allows a switch in reactivity with respect to the corresponding homogeneous processes, thus developing new advantageous synthetic protocols [18,19]. 
However, in some cases the true nature of catalytic species (heterogeneous vs. homogeneous) is unclear: supported metal catalysts, which are described in the literature as merely heterogeneous, can actually show a significant loss of their activities when reused, due to the metal leaching occurring during the reaction [20]. The problem of distinguishing heterogeneous from homogeneous catalysis is far from trivial: careful investigations such as hot filtration and recyclability tests, kinetic studies and selective catalysts poisons are very helpful to solve the question of heterogeneity.

To the best of our knowledge, a comprehensive and up-to-date overview of all the synthetic strategies for the preparation of $N$-heterocycles by supported metal catalysts is still missing in the literature. In fact, only sectorial mini-reviews have been reported so far, focused on some specific types of supports-nanomaterials [21-23], magnetic matrices [24-27], metal oxides [28,29], carbon materials [30,31] - or on selected classes of reactions and heterocycles [32-35]. Therefore, in this review we will give a critical overview of all the solid supported metal catalysts applied in the synthesis of $N$-heterocycles. In particular, we will mostly follow a systematic approach, organizing the manuscript in three parts as a function of the type of support: (i) metal catalysts supported on inorganic matrices (carbon, silica, metal oxides, minerals, magnetic core/inorganic shell composites); (ii) metal catalysts supported on organic matrices (polymers, polymer-anchored ligands, biopolymers); (iii) metal catalysts supported on hybrid inorganic-organic matrices (carbon-anchored ligands, silica-anchored ligands, metal oxides or minerals-anchored ligands, magnetic core/inorganic shell composite- anchored ligands, magnetic core/organic shell composites, metal organic frameworks).

It is worth pointing out that here we will not consider the synthesis of $N$-heterocycles by using neither semi-heterogeneous or quasi-heterogeneous catalytic systems, nor metal catalysts supported on soluble matrices. In fact, in our critical overview, we will try to emphasize the effective heterogeneity and recyclability of the described metal catalysts, specifying which studies were carried out in order to evaluate these aspects.

\section{Metal Catalysts Supported on Inorganic Matrices for the Synthesis of $N$-Heterocycles}

Inorganic matrices are widely used as support for the development of reusable metal catalysts: these materials are often commercially available at a low cost, showing good stability at high temperatures and pressures, excellent mechanical properties and long durability; moreover, they can be tailor-modified and implemented in the desired morphology, and in some cases are easy to regenerate [36]. A further advantage of inorganic matrices is that they are generally insoluble in organic solvents, thus allowing an easy recovery of the catalyst from the reaction medium by simple filtration.

It is therefore not surprising that over the past 20 years, metal catalysts supported on several inorganic matrices have been widely used for the synthesis of $N$-heterocycles: in addition to the most common charcoal (and related carbon materials), silica (including its mesostructured derivatives) and metal oxides, there are also examples of metal catalysts supported on minerals and, more recently, on magnetic core/inorganic shell composites. In this first section of the paper, we will organize literature following this above-mentioned order.

\subsection{Metal Catalysts Supported on Carbon}

To the best of our knowledge, the first application of a metal catalyst supported on carbon was reported in 2004 by Djakovitch et al.: commercially available palladium on activated carbon $(\mathrm{Pd} / \mathrm{C})$ was successfully applied in several tandem reactions, including a one-pot synthesis of 2-phenylindole (4) by Sonogashira coupling of 2-iodoaniline (1) with phenylacetylene (2), followed by intramolecular heteroannulation of the intermediate 3 to form the final indole 4 [37]. The reaction was performed on a $5 \mathrm{mmol}$ scale with a moderate catalyst loading $(1 \mathrm{~mol} \%$ of $\mathrm{Pd} / \mathrm{C})$, in the presence of $\mathrm{CuI}(1 \mathrm{~mol} \%)$ as the co-catalyst and in DMF $/ \mathrm{H}_{2} \mathrm{O}(1: 1 \mathrm{v} / \mathrm{v})$ as the solvent, at $120{ }^{\circ} \mathrm{C}$ for $6 \mathrm{~h}$, affording indole 4 in $72 \%$ yield (Scheme 1). Recycling experiments of the recoverable $\mathrm{Pd} / \mathrm{C}$ demonstrated 
a rapid deactivation of the catalyst: from $100 \%$ conversion for the first run to $20 \%$ for the second run and $<5 \%$ for the third run. This aspect, together with the use of homogeneous $\mathrm{CuI}$ as the co-catalyst, makes the protocol unattractive from the point of view of Green Chemistry criteria.

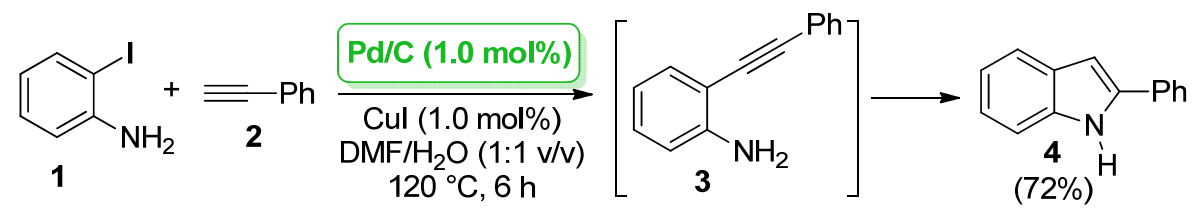

Scheme 1. Synthesis of 2-phenylindole (4) by tandem Sonogashira coupling/intramolecular heteroannulation catalyzed by commercial Pd/C, reported in 2004 by Djakovitch et al.

In 2007, Török and co-workers described the combined use of supported Pd/C and solid-acid K-10 montmorillonite as catalysts for the synthesis of pyrazoles 7 from chalcones 5 and hydrazines 6 via a tandem cyclization-dehydrogenation protocol, performed in only 30 min under microwaves irradiation and solvent-free conditions (Scheme 2) [38]. In particular, the use of solid-acid K-10 montmorillonite allowed the cyclization of chalcones 5 with hydrazines 6, while $\mathrm{Pd} / \mathrm{C}$ is responsible for the dehydrogenation step to give the final product 7. The procedure is very appealing since it complies with most of the Twelve Principles of Green Chemistry, thanks to the use of recoverable catalysts (Principle 1: Prevention of Waste; Principle 4: Designing Safer Chemicals; Principle 8: Catalysis), microwaves irradiation as non-conventional energy source (Principle 6: Design for Energy Efficiency) and solvent-free conditions (Principle 5: Safer Solvents and Auxiliaries).

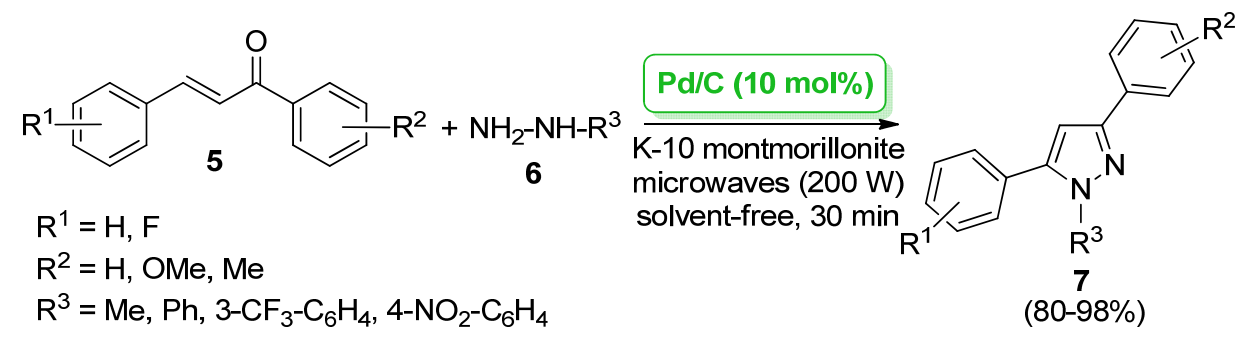

Scheme 2. Synthesis of pyrazoles 7 from chalcones 5 and hydrazines 6 via tandem cyclizationdehydrogenation catalyzed by supported Pd/C, reported in 2007 by Török and co-workers.

The successful combination of the supported Pd/C catalyst with the solid-acid K-10 montmorillonite was also more recently reported by Magolan and co-workers for the synthesis of benzimidazoles 9 from ortho-nitroanilines 8 by tandem transfer hydrogenation/condensation/dehydrogenation [39]. Reactions were performed by treating anilines 8 with $\mathrm{NH}_{4} \mathrm{HCO}_{2}$ (3.3 equiv.) as the hydrogen donor, in the presence of both $\mathrm{Pd} / \mathrm{C}$ and $\mathrm{K}-10$, for $1 \mathrm{~h}$ in refluxing toluene; then, a suitable aldehyde (1.0 equiv.) was added at $\sim 4{ }^{\circ} \mathrm{C}$, and the resulting mixture was left under stirring for $16 \mathrm{~h}$ at room temperature (Scheme 3 ). In particular, the role of $\mathrm{Pd} / \mathrm{C}$ is to promote the starting transfer hydrogenation and the final dehydrogenation steps, while the acidity of montmorillonite is responsible for the condensation step. Compared to the protocol of Török and co-workers, the present study was less convenient from the point of view of sustainability, as it required a toxic solvent (i.e., toluene), high thermal heating and long reaction times. Moreover, although this study emphasized the benefits of using solid and recoverable catalysts, authors did not investigate their reusability in recycling tests, nor the heterogeneity of $\mathrm{Pd} / \mathrm{C}$ by hot filtration tests, thus casting doubts on the real sustainability of this protocol. 


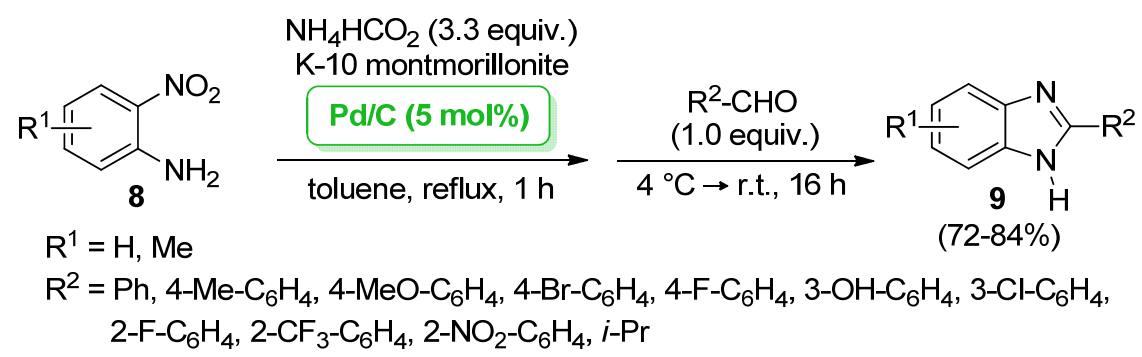

Scheme 3. Synthesis of benzimidazoles 9 from ortho-nitroanilines $\mathbf{8}$ by tandem transfer hydrogenation/condensation/dehydrogenation catalyzed by Pd/C, reported in 2012 by Magolan et al.

Interestingly, also bimetallic palladium-copper supported on charcoal (Pd-Cu/C) was successfully applied as a recoverable catalyst for the synthesis of $N$-heterocyclic compounds. Felpin and co-workers reported in 2014 the use of bimetallic $\mathrm{Pd}-\mathrm{Cu} / \mathrm{C}$ as a heterogeneous multi-task catalyst for the synthesis of triazoles by Sonogashira coupling of iodoarenes with trimethylsilylacetylene, followed by one-pot sequential desilylation and [3 + 2] cycloaddition with suitable azides [40]. Although authors pointed out the heterogeneity of the catalytic system, some recycling tests performed on a representative Sonogashira/click reaction revealed that $\mathrm{Pd}-\mathrm{Cu} / \mathrm{C}$ was almost inactive after the second run; moreover, ICPMS analyses of the filtered solution after the second cycle showed a marginal leaching of $\mathrm{Pd}(<0.2 \mathrm{wt} . \%)$ but a severe $\mathrm{Cu}$ leaching ( $16 \mathrm{wt} . \%)$. Such results casted serious doubts on the real sustainability and actual benefits of this method.

However, copper-on-charcoal $(\mathrm{Cu} / \mathrm{C})$ is definitely the most common carbon-supported metal catalyst to be applied in the context of the synthesis of $N$-heterocyclic compounds, especially for the preparation of 1,2,3-1H-triazoles via [3 + 2] cycloaddition reactions. The first study was reported in 2006 by Lipshutz et al.: a nanoparticle-sized Cu/C, bearing both $\mathrm{CuO}$ and $\mathrm{Cu}_{2} \mathrm{O}$ as metal species within the carbon matrix, was prepared by the impregnation of activated wood charcoal with $\mathrm{Cu}\left(\mathrm{NO}_{3}\right)_{2}$ in water following drying at $120{ }^{\circ} \mathrm{C}$ under vacuum; it was then successfully used in the click reactions of benzyl, homobenzyl or adamantyl azides with several terminal alkynes, performed in dioxane at $60{ }^{\circ} \mathrm{C}$ and in the presence of $\mathrm{Et}_{3} \mathrm{~N}$ (1.0 equiv.), to give the corresponding triazoles in a few minutes and high yields (>90\%) [41]. In a following study, they applied the same $\mathrm{Cu} / \mathrm{C}$ catalyst in a tandem diazo transfer/click reaction protocol: primary amines $\mathbf{1 0}$ were first treated with trifluoromethansulfonyl azide $\left(\mathrm{TfN}_{3}, 1.0\right.$ equiv.) and $\mathrm{Ba}(\mathrm{OH})_{2}(1.0$ equiv.), in the presence of $\mathrm{Cu} / \mathrm{C}(12 \mathrm{~mol} \%)$ and in $\mathrm{CH}_{2} \mathrm{Cl}_{2}$ as the solvent, to give the corresponding azide intermediate 11; then, alkynes (0.5 equiv.) and $\mathrm{Et}_{3} \mathrm{~N}$ (0.9 equiv.) were added to one pot, giving the final 1,2,3-1H-triazoles 12 in most cases in high yields (Scheme 4) [42].

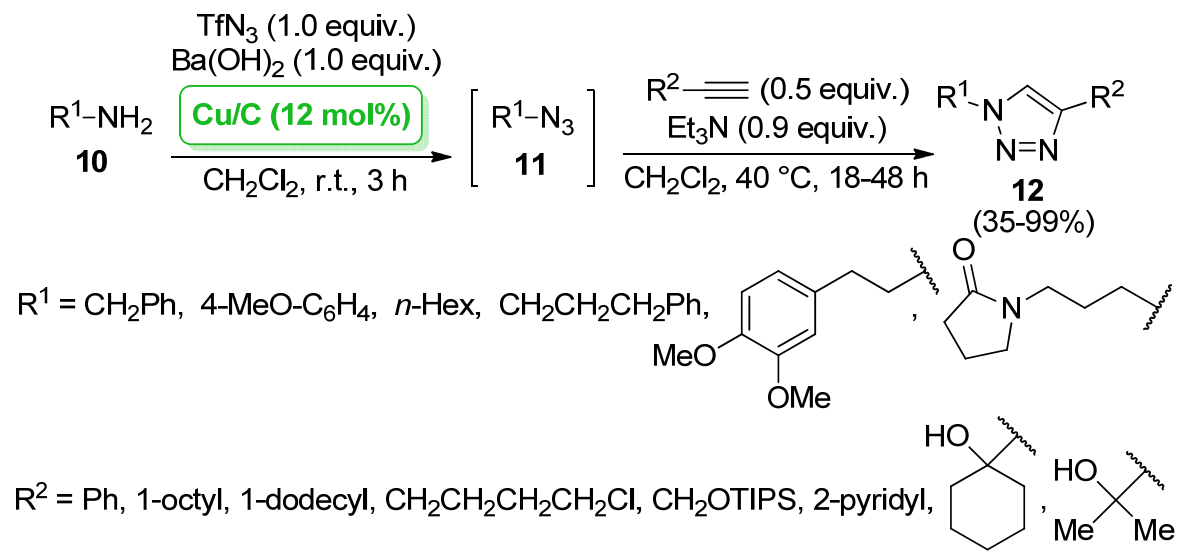

Scheme 4. Synthesis of 1,2,3-1H-triazoles 12 by tandem diazo transfer/[3 + 2] cycloaddition of primary amines 10 catalyzed by nanoparticle-sized $\mathrm{Cu} / \mathrm{C}$, reported in 2009 by Lipshutz and co-workers. 
In 2010, Alonso, Yus and co-workers described the use of copper nanoparticles supported on carbon (CuNPs/C) as a catalyst for the synthesis of 1,2,3-triazoles 17-19 by three- component 1,3-dipolar cycloadditions of suitable organic azide precursors (bromides 13, diazonium salts 14, anilines 15 or epoxides 16), with $\mathrm{NaN}_{3}$ and terminal alkynes in water as the solvent, at $70-100{ }^{\circ} \mathrm{C}$ for $2-7 \mathrm{~h}$ (Scheme 5) [43]. A more extended study was performed in 2011 by the same authors, demonstrating the high stability and recyclability of CuNPs/C: the catalyst was used up to five runs without significant loss in the yield of triazole products; moreover, ICP-MS analyses of the aqueous reaction mixture after catalyst removal by filtration revealed low amounts $(<50 \mathrm{ppb})$ of $\mathrm{Cu}$ species leached into the solution, thus confirming that the catalytic process occurred through a merely heterogeneous mechanism [44].

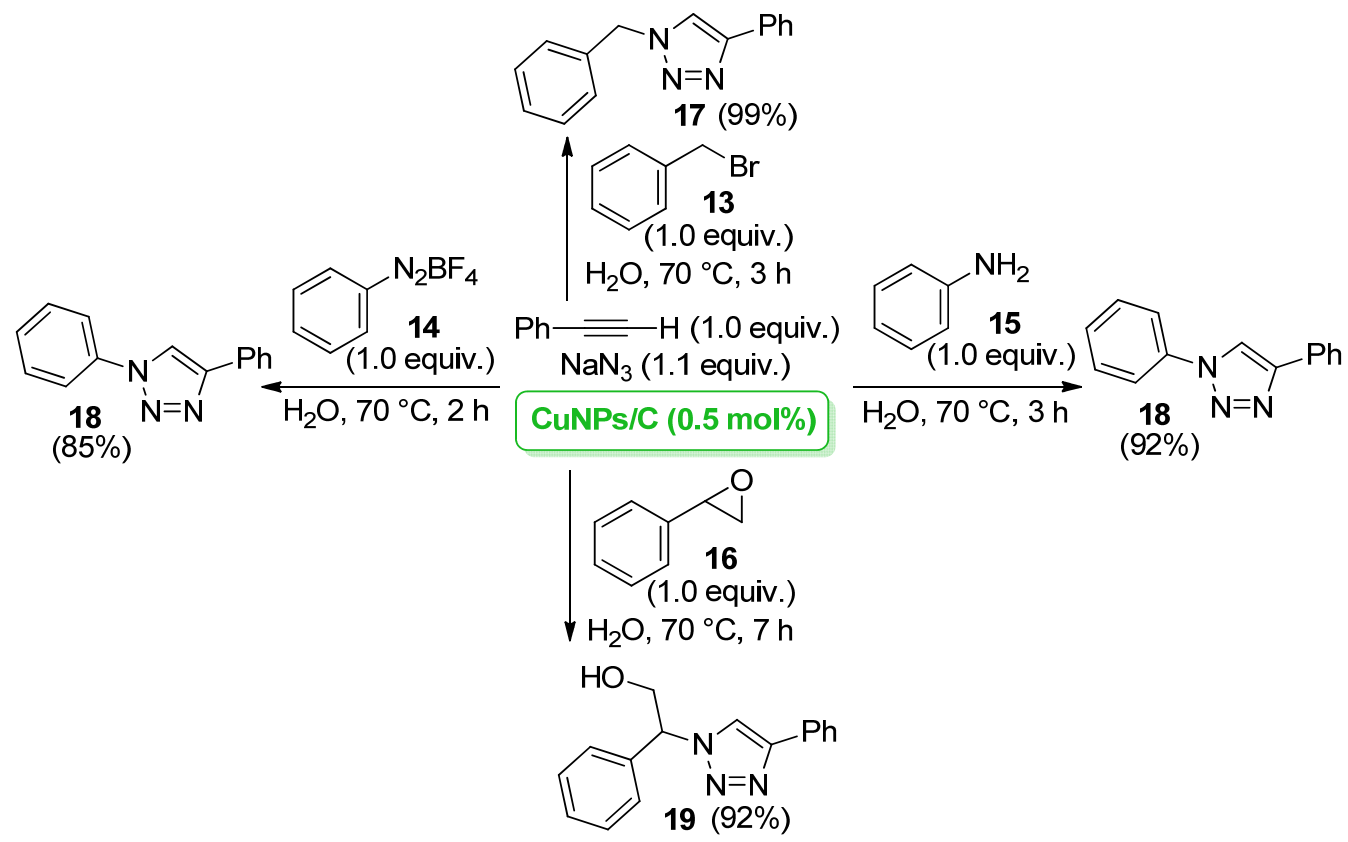

Scheme 5. Synthesis of 1,2,3-triazoles 17-19 by three-component 1,3-dipolar cycloadditions of suitable organic azides precursors (bromides 13 , diazonium salts 14 , anilines 15 or epoxides 16) catalyzed by $\mathrm{CuNPs} / \mathrm{C}$, reported in 2010 by Alonso, Yus and co-workers.

More recently, the same group reported the use of CuNPs/C as the supported catalyst for the synthesis of indolizines $\mathbf{2 3}$ by multicomponent reaction of pyridine-2-carbaldehyde (20) with secondary amines 21 and terminal alkynes 22 (mostly aryl acetylenes) [45]. Reactions were performed at $70{ }^{\circ} \mathrm{C}$ in $\mathrm{CH}_{2} \mathrm{Cl}_{2}$ using $0.5 \mathrm{~mol} \%$ of the copper catalyst, affording the desired indolizines $\mathbf{2 3}$ in moderate-to-high yields (Scheme 6). Contrary to the good recyclability observed for CuNPs/C in the abovementioned three-component 1,3-dipolar cycloadditions, in this case Alonso and co-workers observed a severe catalyst deactivation after the first run, due to metal leaching and/or catalyst poisoning.

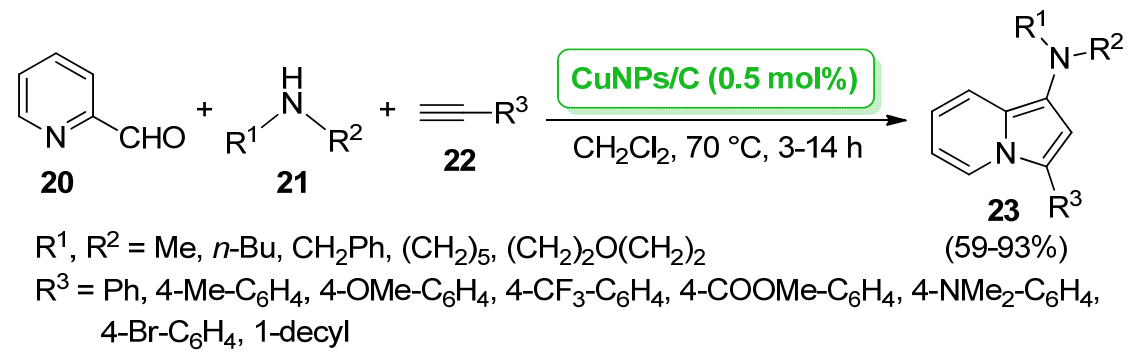

Scheme 6. Synthesis of indolizines 23 by multicomponent reaction of pyridine-2-carbaldehyde (20) with amines 21 and alkynes 22 catalyzed by CuNPs/C, reported in 2013 by Alonso et al. 
The use of a carbon-supported platinum catalyst $(\mathrm{Pt} / \mathrm{C})$ was reported in 2016 by Shimizu and co-workers for the synthesis of 2,5-disubstituted pyrroles 26 by dehydrogenative condensation of 1,2-aminoalcohols 24 with secondary alcohols 25: reactions were performed in diglyme as the solvent, at $125{ }^{\circ} \mathrm{C}$ for $18 \mathrm{~h}$, in the presence of potassium t-butoxide (1.1 equiv.) as the base and $0.1 \mathrm{~mol} \%$ of the Pt/C catalyst (Scheme 7) [46]. Interestingly, recycling tests of the supported $\mathrm{Pt} / \mathrm{C}$ catalyst revealed its high reusability (four runs with no loss of activity), while its heterogeneity was testified by ICP-AES analysis of the filtered reaction mixture after the first run, showing a platinum content leached into the solution below the detection limit. More recently, Fonseca, Pérez Mayoral and co-workers instead described the preparation of 2,3-dihydro-1H-1,5-benzodiazepine from $o$-phenylendiamine and acetone, catalyzed by zirconia or sulfated zirconia supported on two carbon materials with different textural properties (microporous Norit RX3 and mesoporous xerogel) [47]. Although authors emphasized in several points that their protocol was appealing from the point of view of Green Chemistry (as it was performed under solvent-free conditions), unfortunately no investigation on the effective heterogeneity or recyclability of the supported catalysts was carried out.

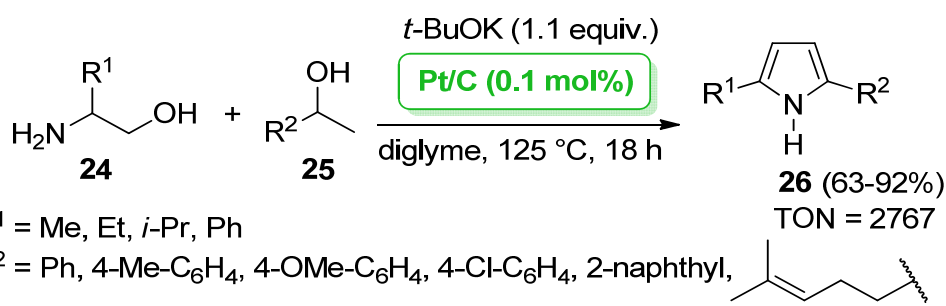

Scheme 7. Synthesis of pyrroles 26 by dehydrogenative condensation of 1,2-aminoalcohols 24 with secondary alcohols 25 catalyzed by Pt/C, reported in 2016 by Shimizu and co-workers.

In addition to (activated) charcoal, several other carbon-based materials have been successfully adopted as a support for metal catalysts: graphene and graphene oxide, carbon nanotubes, fly ash, carbon nitride or other nitrogen-doped carbon materials. These catalysts then found interesting applications in several organic transformations, including the preparation of $N$-heterocyclic compounds.

In 2014, Binder and co-workers reported the development of highly dispersed copper(I) nanoparticles immobilized onto graphene oxide, TRGO/Cu(I): a graphene oxide dispersion in $\mathrm{H}_{2} \mathrm{O}$ was treated overnight with copper(II) acetate under stirring and then reduced under argon at $600{ }^{\circ} \mathrm{C}$ [48]. The obtained material was then used in representative tests of [3+2] cycloadditions of benzyl azide 27 with alkynes $\mathbf{2 8}$ for the synthesis of 1,2,3-1H-triazoles 29, performed with $2 \mathrm{~mol} \%$ of copper loading in THF, for $48 \mathrm{~h}$ at $40{ }^{\circ} \mathrm{C}$ (Scheme 8). Although in recyclability tests TRGO/Cu(I) revealed a significant decrease $(\sim 30 \%)$ of triazole yields after four runs, this was ascribable to the agglomeration of copper nanoparticles, as confirmed by TEM microscopy images, rather than to metal leaching into the solution.

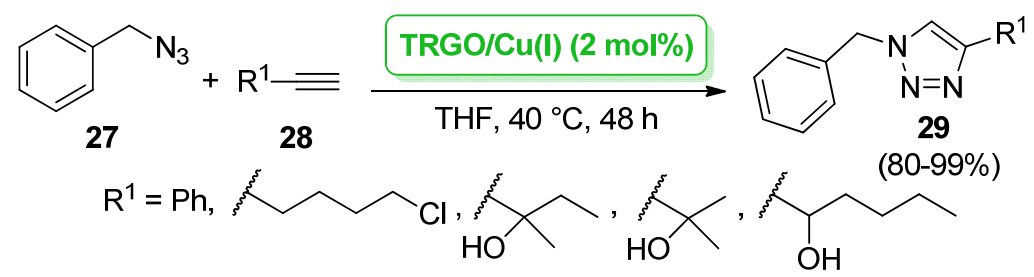

Scheme 8. Synthesis of 1,2,3-1H-triazoles 29 by [3 + 2] cycloadditions of benzyl azide 27 with alkynes 28 catalyzed by TRGO/Cu(I), reported in 2014 by Binder and co-workers.

Das et al. instead reported the preparation of several copper catalysts supported on reduced graphene oxide (that is, $\mathrm{Cu}(0) / \mathrm{rGO}, \mathrm{CuO} / \mathrm{rGO}$ and $\mathrm{CuS} / \mathrm{rGO}$ ) and the evaluation of their activity as recoverable catalysts for the preparation of imidazo[1,2-a]pyridines 
33 via a one-pot, three-component reaction of 2-aminopyridines 30, benzaldehydes 31 and terminal alkynes 32 [49]. In particular, $\mathrm{CuO} / \mathrm{rGO}$ showed the best performance due to its smaller average particle diameter (5 $\pm 1.43 \mathrm{~nm}$, compared to $12 \pm 4.82 \mathrm{~nm}$ of $\mathrm{Cu}(0) / \mathrm{rGO}$ and $183 \pm 48.51 \mathrm{~nm}$ of $\mathrm{CuS} / \mathrm{rGO}$ ), evaluated by TEM analysis. Under optimized conditions, reactions were carried out in DMSO at $110^{\circ} \mathrm{C}$ for $6 \mathrm{~h}$, using 1.0 equiv. of 2-aminopyridines 30, 1.2 equiv. of aldehydes 31, 1.2 equiv. of alkynes 32 and $2.8 \mathrm{~mol} \%$ of the copper catalyst, affording imidazo[1,2-a]pyridines 33 in 89-95\% yields (Scheme 9). A hot filtration test proved the heterogeneity of $\mathrm{CuO} / \mathrm{rGO}$ catalyst in this one-pot, three-component reaction, while recyclability studies confirmed that it can be used up to five times without loss of activity.

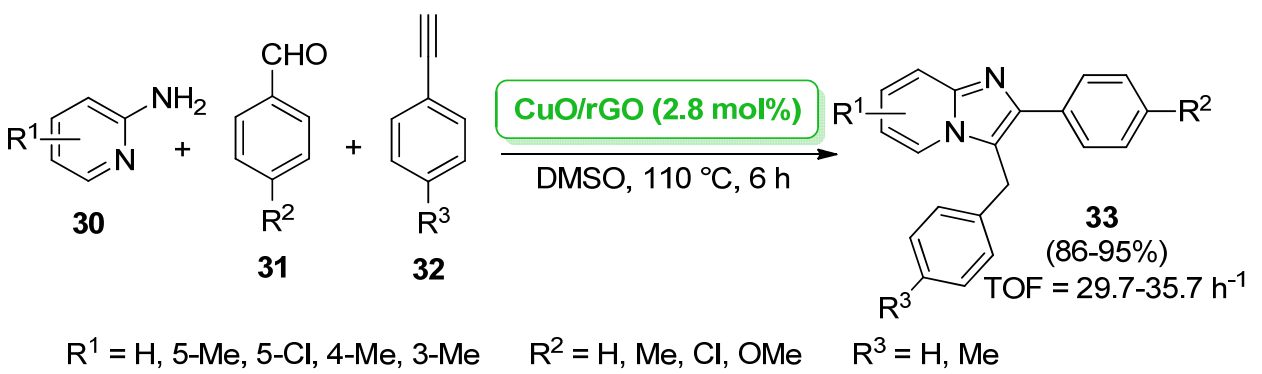

Scheme 9. Synthesis of imidazo[1,2-a]pyridines 33 by three-component reaction of 2-aminopyridines 30, benzaldehydes 31 and terminal alkynes 32 catalyzed by CuO/rGO, reported in 2017 by Das et al.

A trimetallic supported catalyst, consisting of highly monodisperse palladium, ruthenium and nickel nanoparticles furnished with graphene oxide (named PdRuNi@GO), was prepared in 2016 by Sen, Kilbas and co-workers and used as a stable and highly reusable catalytic system for the synthesis of 1,4-dihydropyridines by multicomponent condensation of an aldehyde (typically benzaldehydes) with 2.0 equiv. of a $\beta$-keto ester and a nitrogen surrogate such as ammonium acetate [50]. Despite the good recyclability of PdRuNi@GO, the need for toxic DMF as a reaction medium limited the interest of the protocol from the Green Chemistry point of view.

In 2012, Mandal et al. described the preparation of palladium nanoparticles onto single-wallet carbon nanotubes (SWNT-PdNPs): nanotubes were functionalized with Pd nanoparticles by pyrolysis of $\mathrm{Pd}(\mathrm{OAc})_{2}$ under inert atmosphere, in order to avoid the use of hazardous reducing agents [51]. The SWNT-PdNPs nanocomposite was successfully applied in the synthesis of pyrimidin-2-amines $\mathbf{3 6}$ through the $\mathrm{Cu}$-free acyl Sonogashira step of acid chlorides 34 with trimethylsilylacetylene, performed for $12 \mathrm{~h}$ at room temperature in dry $\mathrm{CH}_{3} \mathrm{CN}$ and in the presence of $\mathrm{Et}_{3} \mathrm{~N}$, followed by treatment with guanidine hydrochloride 35 (2.5 equiv.) in the presence of $\mathrm{Na}_{2} \mathrm{CO}_{3}$ (3.5 equiv.) as the base (Scheme 10). Although recyclability tests were carried out on a simple acyl Sonogashira reaction, SWNT-PdNPs showed good reusability for seven runs, although a slight decrease in catalytic activity was observed from the fifth cycle.

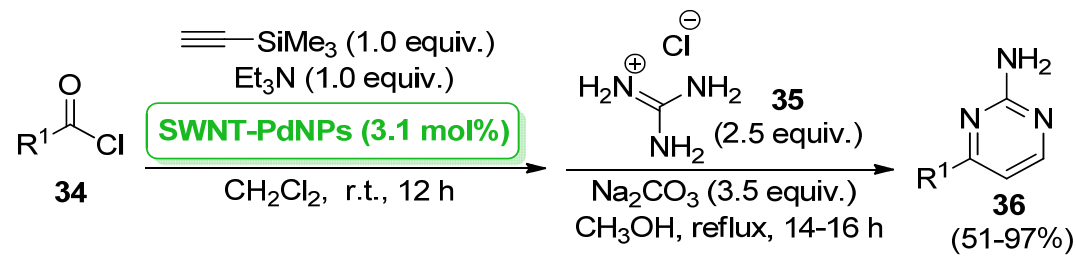

$\mathrm{R}^{1}$ = 2-thienyl, 2-furyl, 1-naphthyl, 2-naphthyl, cynnamyl, 1-adamantyl, $\mathrm{Ph}, t$-Bu

Scheme 10. Synthesis of pyrimidin-2-amines 36 by acyl Sonogashira reaction of acid chlorides 34 with trimethylsilylacetylene followed by treatment with guanidine hydrochloride 35 catalyzed by SWNT-PdNPs, reported in 2012 by Mandal et al. 
Recently, Pérez Mayoral et al. reported the development of novel eco-sustainable catalysts based on $\mathrm{CoO}$ nanoparticles deposited on carbon supports with different morphologies and textures, including multiwall carbon nanotubes (MWCNTs) [52]. Although all these materials were tested as catalysts for the synthesis of quinolines by Friedländer condensation from 2-aminobenzaldehydes and ethyl acetoacetate under solvent-free conditions, the best performance was obtained by $3 \mathrm{wt}$ \% CoO nanoparticles on MWCNTs.

Compared to activated carbon and other typical carbon-based supports, fly ash is an inexpensive material, typically obtained as a pulverized by-product of coal burning. Interestingly, Krishnasamy, Swaminathan and co-workers in 2019 reported the development of a fly ash-supported bimetallic $\mathrm{Bi}_{2} \mathrm{O}_{3}-\mathrm{ZnO}$ catalyst, which was successfully applied in the one-pot synthesis of 1,2,4,5-tetra-substituted imidazoles 40 by a condensation-cyclization reaction of (hetero)aryl aldehydes 37, benzyl 38 and $N$-(2-aminoethyl)pyrrolidine 39, performed in the presence of ammonium acetate and in ethanol as the solvent, at $80{ }^{\circ} \mathrm{C}$ for $9 \mathrm{~h}$ (Scheme 11) [53]. The $\mathrm{Bi}_{2} \mathrm{O}_{3}-\mathrm{ZnO} /$ fly ash catalyst showed moderate recyclability, although a $\sim 20 \%$ decrease in the imidazole yield was observed after the fourth run; however, no hot filtration test or ICP analysis was performed in order to evaluate the amount of metal leaching into the solution.

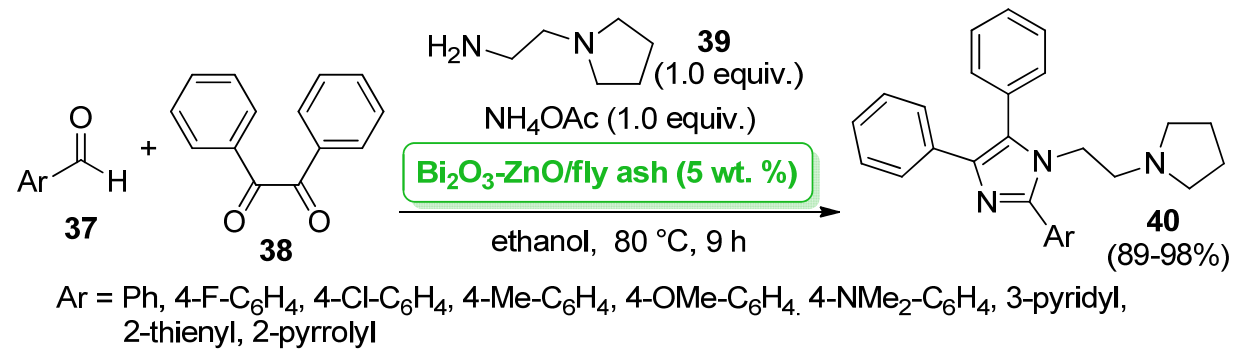

Scheme 11. Synthesis of imidazoles 40 by one-pot condensation-cyclization of (hetero)aryl aldehydes 37, benzyl 38 and $N$-(2-aminoethyl)pyrrolidine 39 catalyzed by $\mathrm{Bi}_{2} \mathrm{O}_{3}-\mathrm{ZnO} /$ fly ash, reported in 2019 by Krishnasamy, Swaminathan and co-workers.

The interest in carbon nitride and other analogous nitrogen-doped carbon materials as support for metal catalysts has been developed in the last few years. In 2018, Dabiri et al. reported the incorporation of $\mathrm{Cu}$ nanoparticles on the surface of a mesoporous carbon nitride ( $\mathrm{MCN}$, prepared using mesoporous SBA-15 silica as a template) support, by treatment with $\mathrm{Cu}\left(\mathrm{NO}_{3}\right)_{2}$ in the presence of ascorbic acid as the reducing agent [54]. The obtained Cu NPs-MCN nanocomposite was then successfully used in several representative examples of the multicomponent 1,3-dipolar cycloaddition reaction of organic bromides, phenylacetylene and $\mathrm{NaN}_{3}$, carried out at low copper loading $(0.5 \mathrm{~mol} \%)$ in a $\mathrm{H}_{2} \mathrm{O} /$ ethanol $(1: 1 \mathrm{v} / \mathrm{v})$ mixture, at $60{ }^{\circ} \mathrm{C}$ for $10 \mathrm{~h}$. The protocol seems to respect several principles of Green Chemistry (in particular Principle 2: Atom Economy; Principle 5: Safer Solvents and Auxiliaries; Principle 8: Catalysis), and the recyclability of Cu NPs-MCN was proven up to eight runs without a loss of activity.

More recently, Bhanage and co-worker instead reported the use of urea-derived porous graphitic carbon nitride (U-g- $\mathrm{C}_{3} \mathrm{~N}_{4}$ ) as a highly stable and biocompatible support for copper nanoparticles: the resulting $\mathrm{Cu} @ \mathrm{U}-\mathrm{g}-\mathrm{C}_{3} \mathrm{~N}_{4}$ was then tested as a heterogeneous and recyclable catalyst in a green and sustainable route for the synthesis of benzimidazoles 42 by the reaction of ortho-phenylenediamines 41 with $\mathrm{CO}_{2}$ as a $\mathrm{C}_{1}$ carbon source and dimethylamine borane (DMAB) as hydrogen source [55]. Reactions were performed by the treatment of diamine 41 with DMAB (3.0 equiv.) and $\mathrm{Cu} @ \mathrm{U}-\mathrm{g}-\mathrm{C}_{3} \mathrm{~N}_{4}$ catalyst $(1.9 \mathrm{~mol} \%$ of $\mathrm{Cu}$ loading) under $\mathrm{CO}_{2}$ atmosphere $(2.5 \mathrm{MPa})$, in a Polarclean/water $(2: 1 \mathrm{v} / \mathrm{v})$ mixture as the solvent, at $100{ }^{\circ} \mathrm{C}$ for $24 \mathrm{~h}$, affording the desired benzimidazoles 42 in good yields (Scheme 12). Although no hot filtration tests or other analyses have been performed to evaluate copper leaching, $\mathrm{Cu} @ \mathrm{U}-\mathrm{g}-\mathrm{C}_{3} \mathrm{~N}_{4}$ exhibited a good recyclability, with only a slight decrease in the yield of benzimidazoles after five runs. This aspect, together with the use 
of a non-toxic, environmentally friendly reaction medium and the appealing opportunity of $\mathrm{CO}_{2}$ fixation through an organic transformation with a high atom economy, makes the protocol of particular interest from the point of view of Green Chemistry.

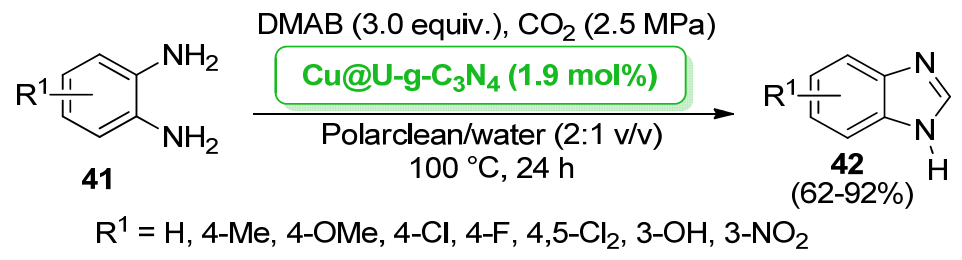

Scheme 12. Synthesis of benzimidazoles 42 by cyclization of ortho-phenylenediamines 41 with $\mathrm{CO}_{2}$ as $\mathrm{C}_{1}$ carbon source catalyzed by $\mathrm{Cu} @ \mathrm{U}-\mathrm{g}-\mathrm{C}_{3} \mathrm{~N}_{4}$, reported in 2019 by Bhanage and co-worker.

Zhang et al. instead reported the use of a nitrogen-doped, carbon-based cobalt catalyst (which they named $\mathrm{Co}-\mathrm{N}_{\mathrm{x}} / \mathrm{C}-800-\mathrm{AT}$ ) for the one-pot heterocyclization of nitro compounds 43 with 2,5-hexadione 44 to give 2,5-dimethyl $N$-substituted pyrroles 45 via the Paal-Knorr condensation process, performed in ethanol as the solvent and in the presence of an excess (4.5 equiv.) of formic acid as both the hydrogen donor and acid co-catalyst (Scheme 13) [56]. Other metal catalysts supported on nitrogen-doped carbon for the synthesis of $N$-heterocycles have been described in the last few years by Yang, Song and co-workers: in 2019, they reported the development of heterogeneous iron nanocomposites supported on $N$-doped porous carbon, used for the preparation of quinazolines by oxidative coupling of 2-aminobenzylamines with aldehydes [57]; in 2020, they instead reported the preparation of highly dispersed ultrafine $\mathrm{Ni}_{2} \mathrm{P}$ nanoparticles supported on $N, P$-codoped biomass-derived porous carbon, which was used as a highly stable catalyst for the synthesis of quinazolines and benzimidazoles via oxidative cross-dehydrogenative coupling of alcohols with, respectively, 2-aminobenzylamines or ortho-phenylenediamines [58].

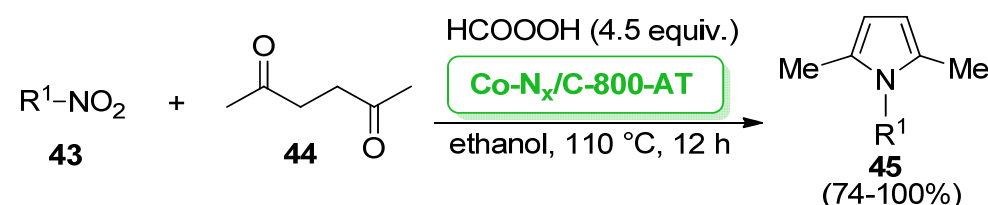

\footnotetext{
$\mathrm{R}^{1}=\mathrm{Ph}, 4-\mathrm{OMe}-\mathrm{C}_{6} \mathrm{H}_{4}, 4-\mathrm{Me}-\mathrm{C}_{6} \mathrm{H}_{4}, 3-\mathrm{Me}-\mathrm{C}_{6} \mathrm{H}_{4}, 2-\mathrm{Me}-\mathrm{C}_{6} \mathrm{H}_{4}, 4-\mathrm{Cl}_{-} \mathrm{C}_{6} \mathrm{H}_{4}, 4-\mathrm{F}-\mathrm{C}_{6} \mathrm{H}_{4}, 4-\mathrm{Br}-\mathrm{C}_{6} \mathrm{H}_{4}$, 4-CHO-C $6 \mathrm{H}_{4}, 2-\mathrm{CHO}-\mathrm{C}_{6} \mathrm{H}_{4}, 4-\mathrm{COMe}_{-} \mathrm{C}_{6} \mathrm{H}_{4}, 4-\mathrm{CH}_{2} \mathrm{OH}-\mathrm{C}_{6} \mathrm{H}_{4}, 4-\mathrm{CN}-\mathrm{C}_{6} \mathrm{H}_{4}$, 4-vinyl- $\mathrm{C}_{6} \mathrm{H}_{4}$, 6-quinolinyl, cyclohexyl, $n$-Bu
}

Scheme 13. Synthesis of 2,5-dimethyl $N$-substituted pyrroles 45 via Paal-Knorr condensation of nitro compounds 43 with 2,5-hexadione 44 catalyzed by Co- $\mathrm{N}_{\mathrm{x}} / \mathrm{C}-800-\mathrm{AT}$, reported in 2017 by Zhang et al.

\subsection{Metal Catalysts Supported on Silica}

To the best of our knowledge, the first application of a metal catalyst supported on silica was described in 2002 by de la Hoz and co-workers, which reported the cyclotrimerization of nitriles 46 to give 1,3,5-triazines 47 using silica-supported $\mathrm{ZnCl}_{2}, \mathrm{AlCl}_{3}$ and $\mathrm{TiCl}_{4}$ as Lewis acid catalysts [59]. Reactions were carried out in solvent-free conditions, by treating nitriles 46 with 1.0 equiv. of anhydrous piperidine as nucleophilic species to induce cyclotrimerization and $2 \mathrm{~mol} \%$ of the $\mathrm{SiO}_{2}$-supported Lewis acid catalyst, under thermal heating $\left(200{ }^{\circ} \mathrm{C}\right.$ for $24 \mathrm{~h}$ ) or under microwave irradiation $(210 \mathrm{~W}$ for $2 \mathrm{~min}$, then $150 \mathrm{~W}$ for $28 \mathrm{~min}$ ), affording triazines 47 from modest to good yields (Scheme 14). Although the protocol seems very convenient from the point of view of Green Chemistry, thanks to the combination of supported metal catalysts with the use of a highly efficient non-conventional energy source (i.e., microwaves) and the absence of any reaction solvent, no studies on the catalysts recyclability and/or heterogeneity were performed. 


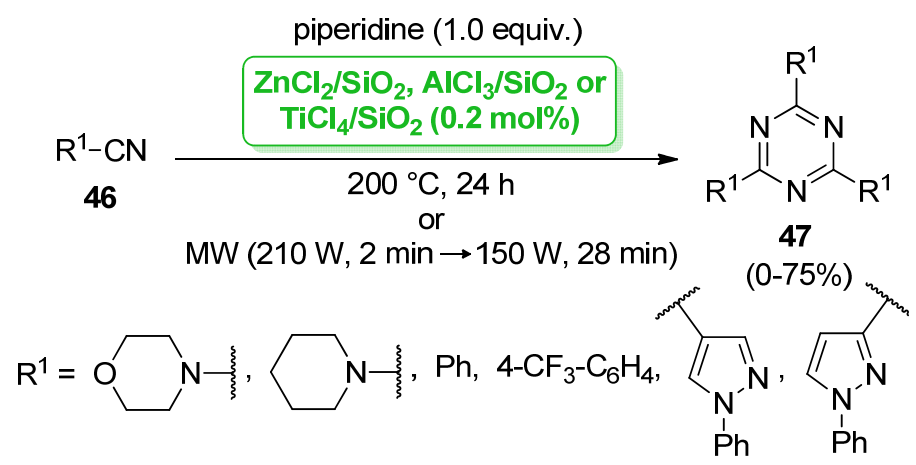

Scheme 14. Synthesis of 1,3,5-triazines 47 via cyclotrimerization of nitriles 46 using silica-supported $\mathrm{ZnCl}_{2}, \mathrm{AlCl}_{3}$ and $\mathrm{TiCl}_{4}$ as Lewis acid catalysts, reported in 2002 by de la $\mathrm{Hoz}$ and co-workers.

However, only in the last decade $\mathrm{SiO}_{2}$-supported metal catalysts have found a more extended application in the synthesis of $N$-heterocycles. In 2012, Soltani Rad, Behrouz et al. reported the preparation of copper-doped silica cuprous sulfate (CDSCS): standard $\mathrm{SiO}_{2}$, after activation under $\mathrm{O}_{2}$ at high temperature, was treated with $\mathrm{ClSO}_{3} \mathrm{H}$ in order to obtain silica sulfuric acid (SSA); to a suspension of the resulting SSA in water, after preliminary ultrasound exposure for $1 \mathrm{~h}$, an excess of $\mathrm{CuI}$ was then added at room temperature to give the final CDSCS material (Scheme 15a) [60]. Interestingly, it was used as a supported catalyst for the [3 +2$]$ cycloaddition reaction of $\beta$-azido alcohols 48 and highly functionalized alkynes 49, performed in a THF $/ \mathrm{H}_{2} \mathrm{O}(1: 1 \mathrm{v} / \mathrm{v})$ mixture and at room temperature, for the synthesis of 1,2,3-1H-triazoles 50 structural analogous of $\beta$-adrenoceptor blocking agents, in short reaction times (up to $1 \mathrm{~h}$ ) and high yields (Scheme 15b). Recyclability tests on CDSCS showed that it could be reused for five consecutive runs without a considerable decrease in its catalytic activity; moreover, the copper amount of CDSCS only marginally decreased during these tests (freshly prepared CDSCS: $6.12 \mathrm{wt.} \%$ vs. CDSCS after fifth run: 6.11 wt. \%; ICP analysis).

In 2013, an easy synthetic route to benzimidazoles, benzothiazoles and benzoxazoles was reported by Mandal et al.: aromatic and aliphatic aldehydes were treated with, respectively, ortho-phenylenediamine, ortho-aminothiophenol and ortho-aminophenol in the presence of silica supported nano-copper (II) oxide (named $\mathrm{CuO}-\mathrm{np} / \mathrm{SiO}_{2}$ ) as the catalyst [61]. The catalytic system showed good recyclability and the absence of any metal leaching into the solution during the cyclization reaction, testified by atomic absorption spectroscopy measurements.

The use of iron oxide nanoparticles supported on silica $\left(\mathrm{Fe}_{3} \mathrm{O}_{4} / \mathrm{SiO}_{2}\right)$ was reported by Maleki for the synthesis of 6,7-dihydro- $1 \mathrm{H}$-1,4-diazepines 53 and 4,5,6,7-tetrahydro- $1 H$-1,4diazepine 55 by one-pot multicomponent reactions: treatment of diamines 51 with terminal alkynes 52 (2.0 equiv.) in the presence of $\mathrm{Fe}_{3} \mathrm{O}_{4} / \mathrm{SiO}_{2}(5 \mathrm{~mol} \%)$, at room temperature and in ethanol as the solvent, afforded diazepine derivatives 53 in good yields in 4-10 h; however, the resulting reaction mixture can also be treated in one pot with cyclohexyl isocyanide (54), giving final tetrahydrodiazepine carboxamides 55 after $6 \mathrm{~h}$ at room temperature (Scheme 16) [62]. The $\mathrm{Fe}_{3} \mathrm{O}_{4} / \mathrm{SiO}_{2}$ catalyst exhibited good recyclability (up to five runs) and the advantage to be magnetically recoverable, although no studies of metal leaching were performed in order to rule out the occurrence of homogeneous iron catalytic species. More recently, Behbahani et al. instead reported the use of $\mathrm{Fe}\left(\mathrm{ClO}_{4}\right)_{3} / \mathrm{SiO}_{2}$ as a supported and recoverable catalyst for the Paal-Knorr synthesis of $N$-substituted pyrroles under solventfree conditions by the reaction of 1,4-dicarbonyl compounds with primary amines [63]. 
(a)
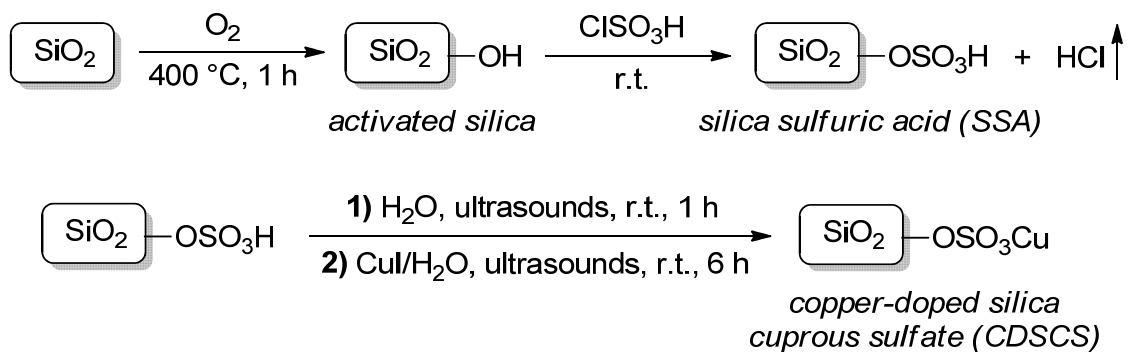

(b)

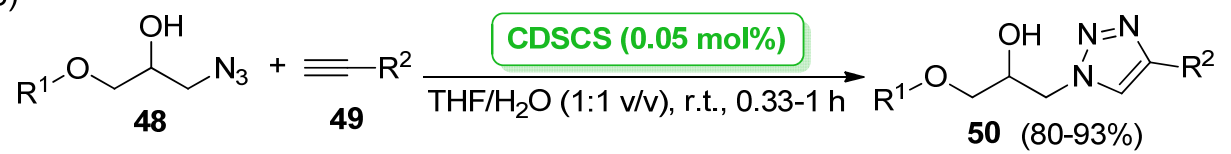

$\mathrm{R}^{1}=$ 2-naphthyl, 4-OMe- $\mathrm{C}_{6} \mathrm{H}_{4}$, 3-Me-4-Cl- $\mathrm{C}_{6} \mathrm{H}_{3}, \mathrm{Ph}, n-\mathrm{Bu}, \mathrm{CH}_{2}-\mathrm{CH}=\mathrm{CH}_{2}, 2,4-\mathrm{Cl}_{2}-\mathrm{C}_{6} \mathrm{H}_{3}, 4-\mathrm{Bn}^{-} \mathrm{C}_{6} \mathrm{H}_{4}$

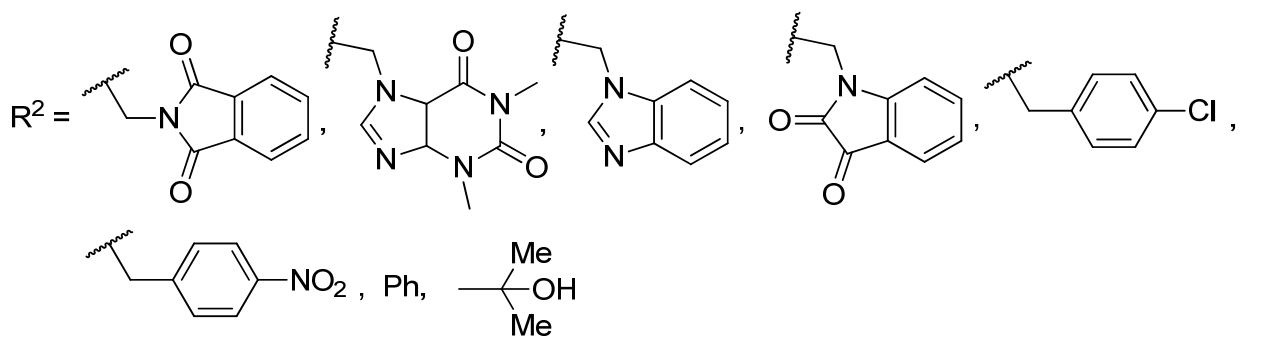

Scheme 15. (a) Schematic route for the preparation of copper-doped silica cuprous sulfate (CDSCS). (b) Synthesis of 1,2,3-1H-triazoles 50 via [3 +2] cycloaddition reaction of $\beta$-azido alcohols 48 and highly functionalized alkynes 49 catalyzed by CDSCS, reported in 2012 by Soltani Rad, Behrouz et al.

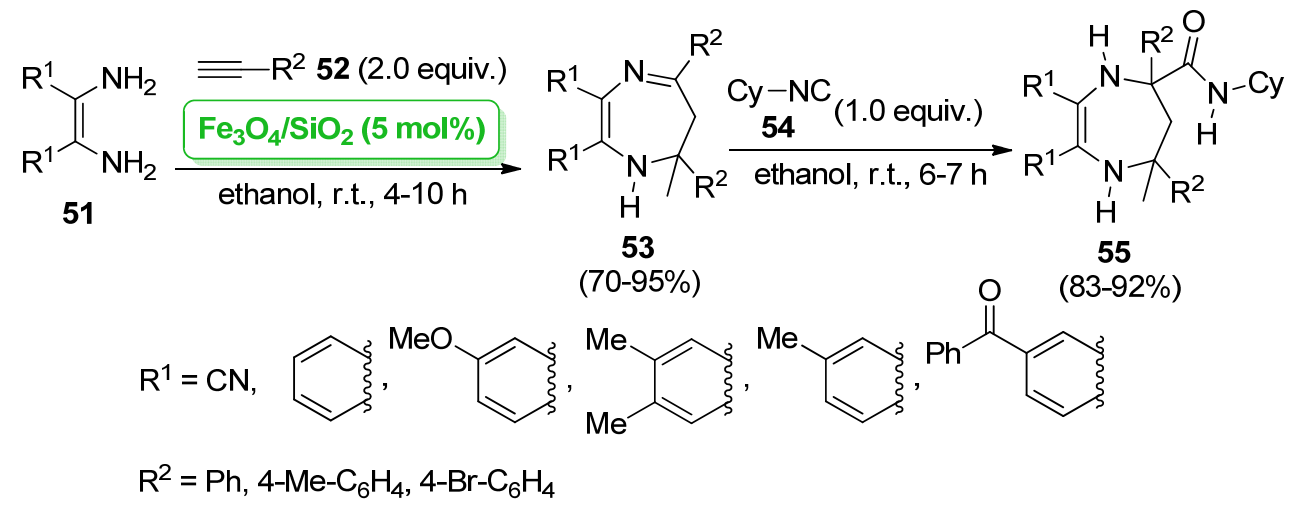

Scheme 16. Synthesis of 6,7-dihydro- $1 H$-1,4-diazepines 53 and 4,5,6,7-tetrahydro- $1 H$-1,4-diazepine 55 by one-pot multicomponent reactions of diamines 51 with alkynes 52 catalyzed by $\mathrm{Fe}_{3} \mathrm{O}_{4} / \mathrm{SiO}_{2}$, reported in 2013 by Maleki.

Safari and co-workers focused their attention on the catalytic properties of antimony trichloride supported on silica gel $\left(\mathrm{SbCl}_{3} / \mathrm{SiO}_{2}\right)$, which was successfully used as a recoverable catalyst in the synthesis of tri-/tetra-substituted imidazoles by one-pot, multicomponent cyclocondensation reactions of benzaldehydes, benzyl and primary amines, in the presence of $\mathrm{NH}_{4} \mathrm{OAc}$ and solvent-free conditions, under conventional thermal heating [64] or microwaves irradiation [65].

In 2015, Jeong and co-workers reported the preparation of a silica-supported cerium ammonium nitrate $\left(\mathrm{CAN}-\mathrm{SiO}_{2}\right)$ by addition of a CAN aqueous solution to a suspension of silica in water [66]. The obtained material was then tested as a heterogeneous catalyst for the synthesis of tetrasubstituted pyrroles 60 through one-pot, four-component cyclocondensation of primary amines 56, (hetero)aryl aldehydes 57, dialkyl acetylenedicarboxylates 58 and nitromethane (59): reactions were performed with an excess of 59, used as both the 
reactant and solvent, in the presence of $10 \mathrm{~mol} \%$ of the $\mathrm{CAN}-\mathrm{SiO}_{2}$ catalyst, under reflux for 4-6 h (Scheme 17). Although $\mathrm{CAN}_{-} \mathrm{SiO}_{2}$ showed excellent recyclability (as it worked for four cycles without a loss of catalytic activity), no studies have been performed to better clarify the occurrence of homogeneous or heterogeneous catalytic species. Moreover, the use of nitromethane (59) as a reaction medium limited the potential impact of this protocol in the context of Green Chemistry.
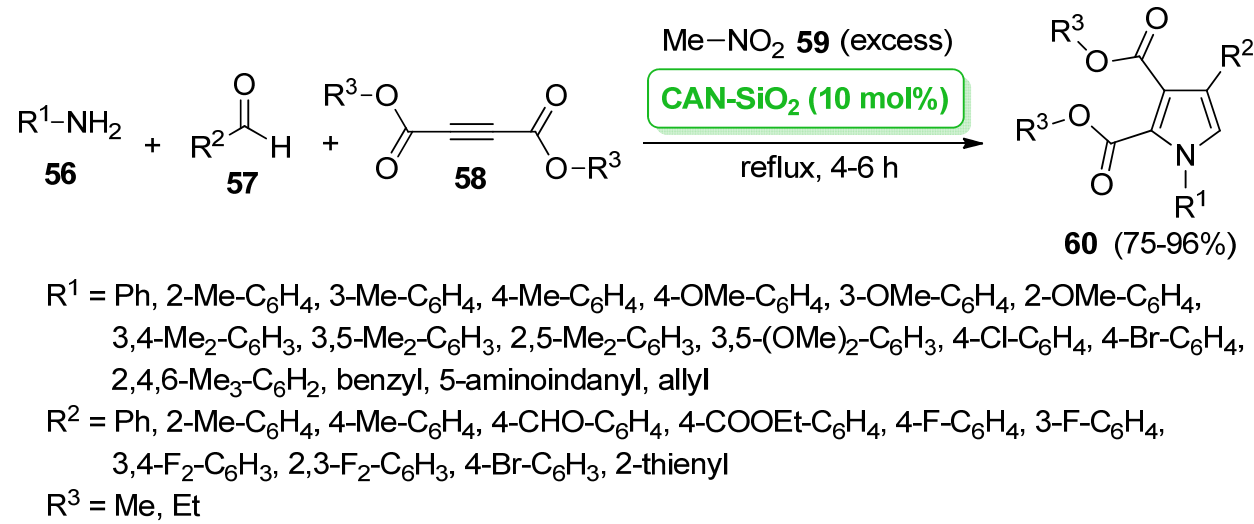

Scheme 17. Synthesis of tetrasubstituted pyrroles 60 by one-pot, four-component cyclocondensation of primary amines 56, (hetero)aryl aldehydes 57, dialkyl acetylenedicarboxylates 58 and nitromethane (59) catalyzed by $\mathrm{CAN}-\mathrm{SiO}_{2}$, reported in 2015 by Jeong and co-workers.

The synthesis of 6-chloro-8-substituted-9H-purines 63 by the reaction of 6-chloropyrimidine 61 with carboxylic acids 62 catalyzed by a silica-supported silver $(\mathrm{Ag} / \mathrm{SiO} 2)$ catalyst was described in 2016 by Lavanya et al. [67]. The synthetic protocol was very appealing from a sustainability point of view, as it complies with most of the Twelve Principles of Green Chemistry: reactions were performed in ethanol as a non-toxic solvent (Principle 5: Safer Solvents and Auxiliaries), at room temperature (Principle 6: Design for Energy Efficiency), with the generation of $\mathrm{H}_{2} \mathrm{O}$ as the only co-product (Principle 1: Prevention of Waste); moreover, the silica-supported silver catalyst (Principle 8: Catalysis) showed high recyclability (five runs with no loss of activity), although the absence of hot filtration tests and/or metal leaching analysis cannot totally exclude the passage of metal species into the solution (Scheme 18).

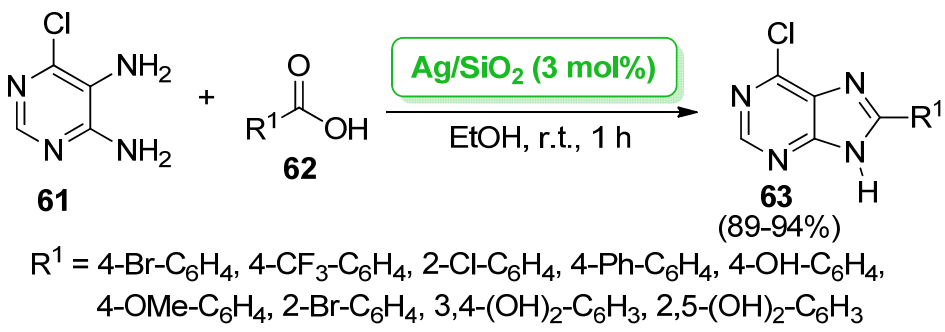

Scheme 18. Synthesis of 6-chloro-8-substituted-9H-purines $\mathbf{6 3}$ by reaction of 6-chloro-pyrimidines $\mathbf{6 1}$ with carboxylic acids 62 catalyzed by $\mathrm{Ag} / \mathrm{SiO}_{2}$, reported in 2016 by Lavanya et al.

In 2011, Che and co-workers developed a silica-supported gold nanoparticles catalyst (AuNPs $/ \mathrm{SiO}_{2}$ ) by the deposition of gold nanoparticles (synthesized in situ from a refluxing solution of $\mathrm{KAuCl}_{4}$ in toluene) onto the surface of a silica support [68]. Interestingly, the obtained material was found to be a very effective catalyst for the oxidative cyclization of anilines 64 with 2.0 equiv. of aldehydes 65 to form highly functionalized quinolines 66 in a one-pot protocol: reactions were carried out in toluene at $110{ }^{\circ} \mathrm{C}$, by bubbling $\mathrm{O}_{2}$ as the oxidant into the reaction mixture and in the presence of $5 \mathrm{~mol} \%$ of $\mathrm{AuNPs} / \mathrm{SiO}_{2}$, affording final quinolines 66 in most cases in high yields (Scheme 19). Although detailed mechanistic 
studies have been carried out, no investigations were performed to clarify the real nature of the catalytically active gold species (that is, homogeneous species leached into the solution vs. heterogeneous species supported on silica); however, $\mathrm{AuNPs} / \mathrm{SiO}_{2}$ was found to be highly recyclable for seven runs, maintaining the same yields of quinoline 66 products.

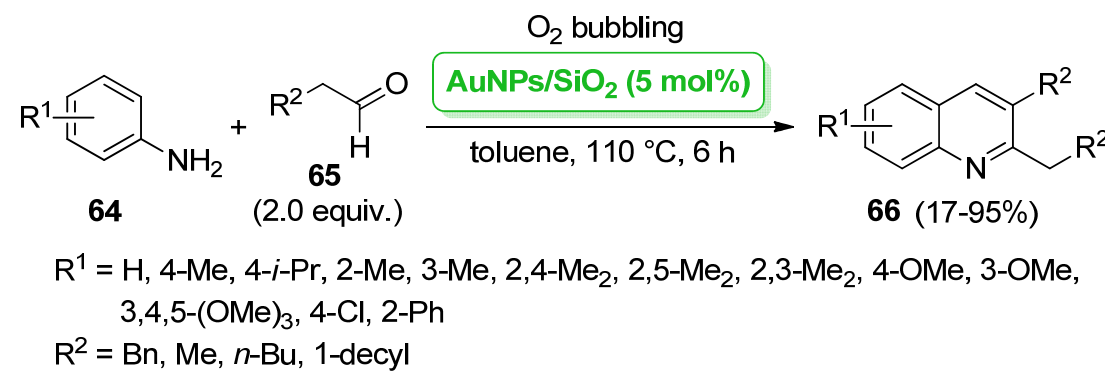

Scheme 19. Synthesis of highly functionalized quinolines 66 by oxidative cyclization of anilines 64 with aldehydes 65 catalyzed by $\mathrm{AuNPs} / \mathrm{SiO}_{2}$, reported in 2011 by Che and co-workers.

More recently, ordered mesoporous silica has attracted growing attention: a plethora of materials (SBA-15, SBA-16, MCM-41, MCM-48, etc.) with a wide range of pore geometries (hexagonal, cubic, etc.) and particle morphologies have been successfully developed for several applications, including as a support for metal catalysts.

In this context, the first use of these catalysts for the synthesis of $N$-heterocycles was reported in 2013 by Abdollahi-Alibeik et al.: mesoporous silica MCM-41 nanoparticles were prepared by the sol-gel method, then boron trifluoride was supported by calcination at different temperatures in order to obtain the final nanostructured solid acid catalyst $\mathrm{BF}_{3} / \mathrm{MCM}-41$ [69]. It was then used (at $20 \mathrm{wt} . \%$ of $\mathrm{BF}_{3}$ loading) for the synthesis of 3-iminoaryl-imidazo [1,2-a] pyridine derivatives 69 by reaction of 2-aminopyridines 67 with 2.0 equiv. of benzaldehydes 68 , in refluxing ethanol and in the presence of trimethylsilyl cyanide (TMSCN, 1.0 equiv.) as a $\mathrm{CN}^{-}$anion source (Scheme 20). An interesting reusability study of the $\mathrm{BF}_{3} / \mathrm{MCM}-41$ catalyst was performed in the model reaction of 2-aminopyridine with benzaldehyde. In four runs, the supported catalyst showed a gradual increase in the reaction time and decrease in the yield of the imidazo[1,2- $a$ ]pyridine product. Since boron leaching from the MCM-41 surface was ruled out $\left(\mathrm{BF}_{3}\right.$ loading of the recovered catalyst was the same as freshly prepared samples, that is, $20 \mathrm{wt} . \%)$, the moderate deactivation was attributable to partial blockage of the catalytically active sites; in fact, calcination $\left(400{ }^{\circ} \mathrm{C}\right.$ for $2 \mathrm{~h}$ ) of the recovered $\mathrm{BF}_{3} / \mathrm{MCM}-41$ catalyst after the fourth run allowed the complete recovery of its initial catalytic activity.

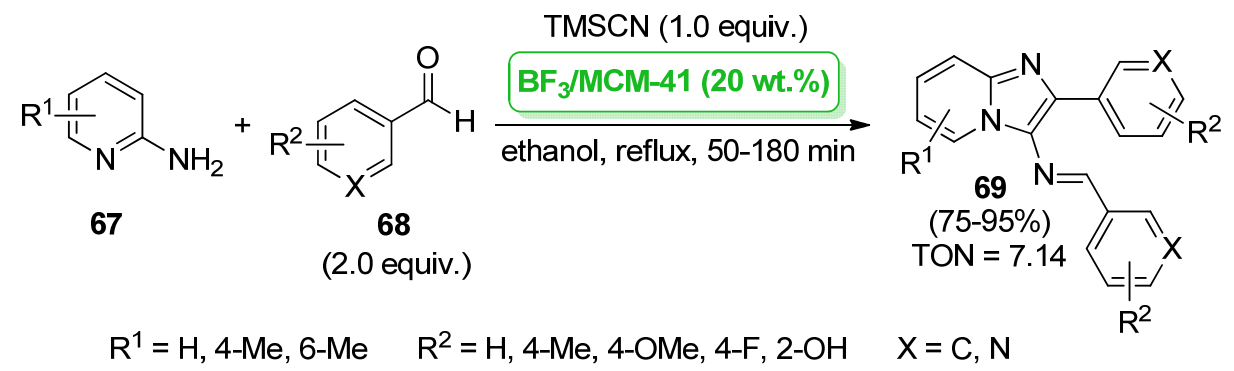

Scheme 20. Synthesis of 3-iminoaryl-imidazo[1,2-a] pyridines 69 by reaction of 2-aminopyridines 67 with benzaldehydes 68 catalyzed by nanostructured solid acid catalyst $\mathrm{BF}_{3} / \mathrm{MCM}-41$, reported in 2013 by Abdollahi-Alibeik et al.

More recently, Biswas and co-workers reported the preparation of rare earth metal catalysts supported on MCM-41 mesoporous silica with large pores (also indicated as l-MSN): in a first study, ytterbium supported on large-pore MCM-41 (Yb@l-MSNs) was prepared by the wet impregnation method of ytterbium triflate and then tested as excel- 
lent catalysts in the synthesis of benzimidazoles through the reaction of aldehydes with $o$-phenylenediamines [70]; in a following investigation, samarium nitrate was impregnated into the l-MSN support to give the Sm@ l-MSNs catalyst, which was explored in the preparation of 5 -substituted $1 \mathrm{H}$-tetrazoles through the reaction of benzonitriles with $\mathrm{NaN}_{3}$, as well as in the preparation of 2-substituted benzothiazoles through the condensation of (hetero)aryl or alkyl aldehydes with 2-aminobenzenethiols [71]. For both catalysts, recyclability and heterogeneity were investigated: if Yb@l-MSNs showed a decrease in catalytic activity after the fourth run, no significant changes were found for Sm@ l-MSNs after five runs; moreover, hot filtration tests and ICP-AES analyses excluded the occurrence of metal leaching into the solution in both cases, thus confirming a merely heterogeneous mechanism.

If functionalization of the silica surface with suitable organic groups is quite common (see Section 4.2 below), its modification with inorganic units is less frequent. In this context, Naeimi et al. reported in 2014 the preparation of phosphorated $\mathrm{SiO}_{2}$ through the treatment of activated silica gel with $\mathrm{PCl}_{3}$ in the presence of triethylamine as a base; the resulting phosphorated $\mathrm{SiO}_{2}$ was then treated with $\mathrm{CuI}$ in acetonitrile at $60^{\circ} \mathrm{C}$ for $24 \mathrm{~h}$, thus affording the final $\mathrm{Cu}(\mathrm{I}) @$ phosphorated $\mathrm{SiO}_{2}$ (named CPSi) catalyst (Scheme 21a) [72]. The present material was successfully applied as a supported catalyst $(0.64 \mathrm{~mol} \%)$ in the threecomponent synthesis of $\beta$-hydroxy-1,2,3-triazoles 72 through the treatment of epoxides 70 with $\mathrm{NaN}_{3}$ and terminal alkynes 71 , at $60^{\circ} \mathrm{C}$ and in water as a solvent (Scheme 21b); after the nucleophilic addition of sodium azide to the starting oxiranes $\mathbf{7 0}$, the resulting $\beta$-hydroxy-azide intermediates gave a one-pot CPSi-catalyzed [3 + 2] cycloaddition with alkynes $\mathbf{7 1}$ affording the final triazoles $\mathbf{7 2}$. Despite the good performance in comparison to other supported copper catalysts, CPSi showed rather limited recyclability, thus limiting their interest from the point of view of Green Chemistry.

(a)

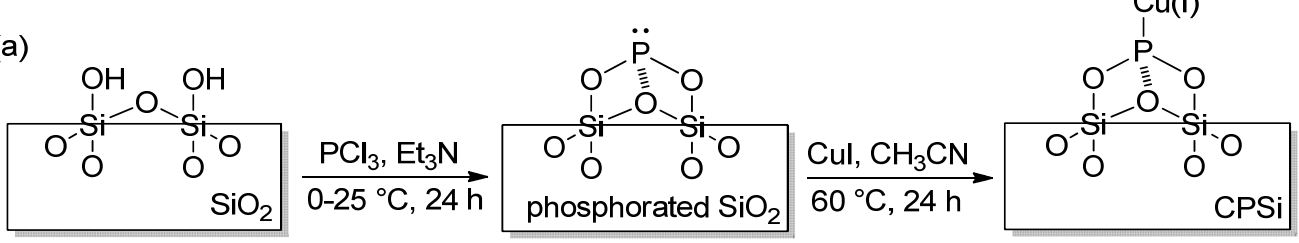

(b)

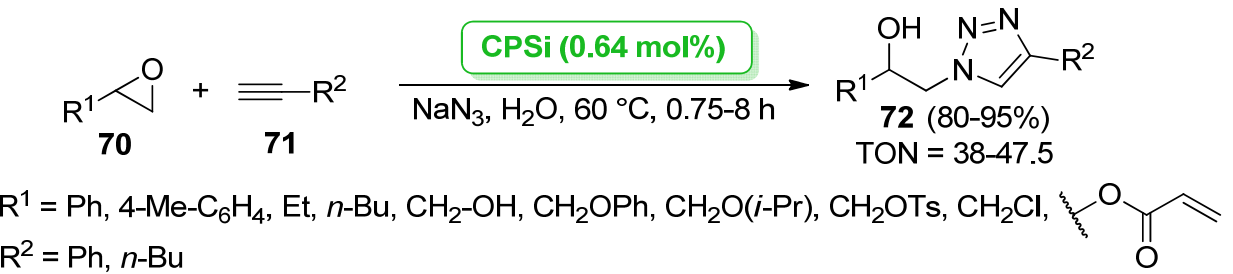

Scheme 21. (a) Schematic route for the preparation of $\mathrm{Cu}(\mathrm{I}) @$ phosphorated $\mathrm{SiO}_{2}(\mathrm{CPSi})$ catalyst. (b) Synthesis of $\beta$-hydroxy-1,2,3-triazoles 72 via three-component reaction of epoxides 70 with $\mathrm{NaN}_{3}$ and terminal alkynes 71 catalyzed by CPSi, reported in 2014 by Naeimi et al.

\subsection{Metal Catalysts Supported on Metal Oxides}

Among all the metal oxides, alumina is definitely one of the most common supports for metal catalysts. In the context of the preparation of $N$-heterocyclic compounds, the first study was reported in 2006 by Razdan and co-workers: a bismuth(III) nitrate immobilized on a neutral alumina $\left(\mathrm{Bi}(\mathrm{III})-\mathrm{Al}_{2} \mathrm{O}_{3}\right)$ catalyst was used for the synthesis of 2,4,6-triarylpyridines from benzylideneacetophenones and (thio)urea derivatives [73]. Although the protocol was carried out under solvent-free conditions, it required a stoichiometric amount of $\mathrm{Bi}(\mathrm{III})-\mathrm{Al}_{2} \mathrm{O}_{3}$, high temperatures $\left(130^{\circ} \mathrm{C}\right)$ and catalyst reactivation (hot air oven, at $110^{\circ} \mathrm{C}$ for $6 \mathrm{~h}$ ) each time before its use.

A very appealing and sustainable synthetic protocol was developed in 2013 by Ranu et al., consisting in the mechanochemical synthesis of 1,2,3-1H-triazole derivatives catalyzed by copper(II) sulfate supported on alumina $\left(\mathrm{Cu} / \mathrm{Al}_{2} \mathrm{O}_{3}\right)$ [74]. On one hand, benzyl 
halides 73 were treated with $\mathrm{NaN}_{3}$ and terminal alkynes 74 into a multicomponent tandem nucleophilic substitution/[3 + 2] cycloaddition to give triazoles 75, performed with $10 \mathrm{~mol} \%$ of $\mathrm{Cu} / \mathrm{Al}_{2} \mathrm{O}_{3}$ under ball milling ( $600 \mathrm{rpm}, 6$ balls) for $1 \mathrm{~h}$. On the other hand, (hetero)aryl boronic acids 76 were first treated with $\mathrm{NaN}_{3}$ (3.0 equiv.), $\mathrm{K}_{2} \mathrm{CO}_{3}$ (1.0 equiv.) and $\mathrm{Cu} / \mathrm{Al}_{2} \mathrm{O}_{3}(10 \mathrm{~mol} \%)$ in ball milling $(600 \mathrm{rpm})$ for $1 \mathrm{~h}$ to give the corresponding organic azides $\mathbf{7 7}$; then, terminal alkynes $\mathbf{7 4}$ were added in a one-pot fashion for the subsequent cycloaddition step, performed again under ball milling $(600 \mathrm{rpm}, 1 \mathrm{~h})$ to give the final 1,2,3-triazole products 75 (Scheme 22). The abovementioned protocol was very convenient, complying with many of the Twelve Principles of Green Chemistry: the use of a cycloaddition reaction, characterized by high atom economy (Principle 2: Atom Economy), under mechanochemical-assisted conditions (Principle 6: Design for Energy Efficiency), which allowed one to work in the absence of solvents (Principle 5: Safer Solvents and Auxiliaries), and in the presence of a recoverable catalyst (Principle 1: Prevention of Waste; Principle 4: Designing Safer Chemicals; Principle 8: Catalysis). Moreover, the $\mathrm{Cu} / \mathrm{Al}_{2} \mathrm{O}_{3}$ catalyst revealed good recyclability: in the representative reaction of benzyl bromide with phenylacetylene, only a slight decrease in the triazole yield was observed after eight runs.

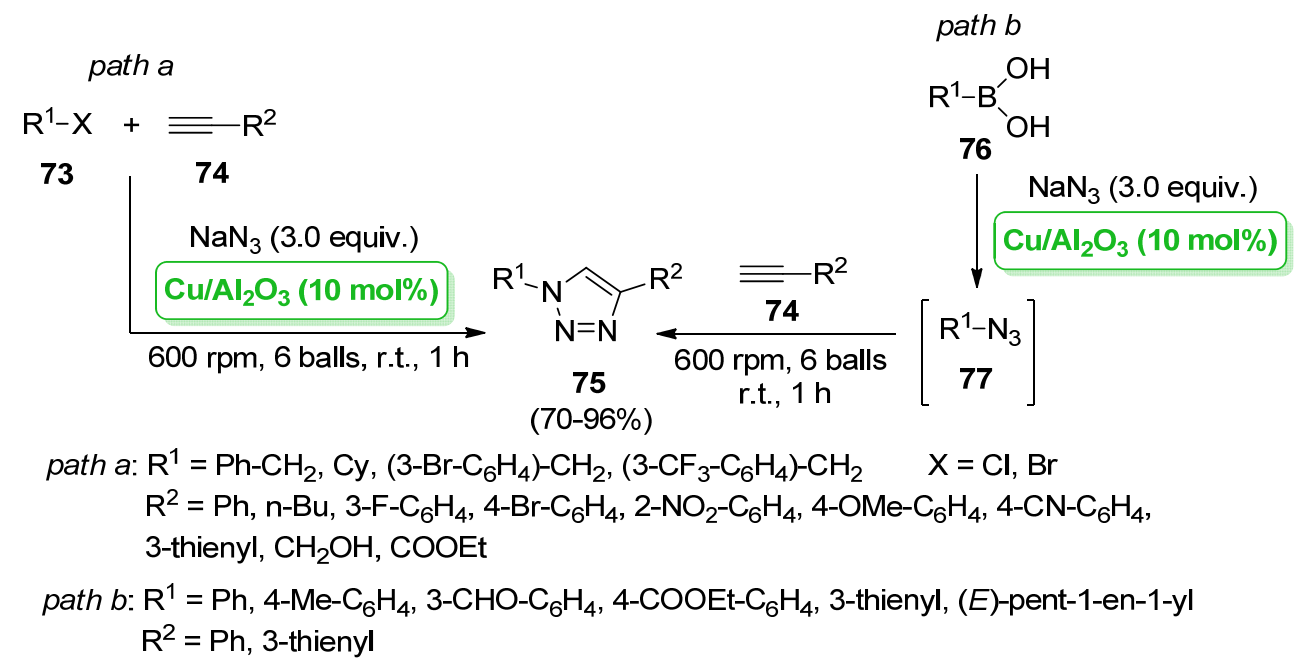

Scheme 22. Synthesis of 1,2,3-triazoles 75 by multicomponent reaction of benzyl halides $73, \mathrm{NaN}_{3}$ and alkynes 74 (path a) or by one pot $\mathrm{S}_{\mathrm{N}} 2 /[3+2]$ cycloaddition of boronic acids $76, \mathrm{NaN}_{3}$ and alkynes 74 (path b) catalyzed by $\mathrm{Cu} / \mathrm{Al}_{2} \mathrm{O}_{3}$, reported in 2013 by Ranu et al.

More recently, a similar multicomponent reaction of benzyl halides with sodium azide and terminal alkynes for the synthesis of 1,4-disubstituted 1,2,3-1H-triazoles, although performed in $\mathrm{H}_{2} \mathrm{O}$ under microwave irradiation or ultrasound sonication as the non-conventional energy source, was developed by Pore and co-workers using neutral alumina-supported copper iodide (named $\mathrm{CuI} / \mathrm{Al}_{2} \mathrm{O}_{3}$ ) as the catalyst [75]. Interestingly, $\mathrm{CuI} / \mathrm{Al}_{2} \mathrm{O}_{3}$ was chosen for its best performance after an extensive screening of homogeneous and heterogeneous copper catalysts, including the above-mentioned copper(II) sulfate on alumina $\left(\mathrm{Cu} / \mathrm{Al}_{2} \mathrm{O}_{3}\right)$.

A bimetallic cerium-vanadium catalyst supported on alumina $\left(\mathrm{Ce}-\mathrm{V} / \mathrm{Al}_{2} \mathrm{O}_{3}\right)$ was developed in 2016 by Lavanya and co-workers for the synthesis of polysubstituted pyridines 80 through a multicomponent reaction of benzaldehydes 78, malonitrile 79 (2.2 equiv.) and ethanol, carried out in $\mathrm{H}_{2} \mathrm{O} / \mathrm{EtOH}(1: 1 \mathrm{v} / \mathrm{v})$ as the solvent, at room temperature for $1 \mathrm{~h}$ (Scheme 23) [76]. Reusability tests of $\mathrm{Ce}-\mathrm{V} / \mathrm{Al}_{2} \mathrm{O}_{3}$ showed a minor loss of the catalytic activity after the fifth run, attributed to poisoning by organic impurities or to structural/morphological changes, rather than to a metal leaching into the solution (although no heterogeneity studies were performed in their work). 


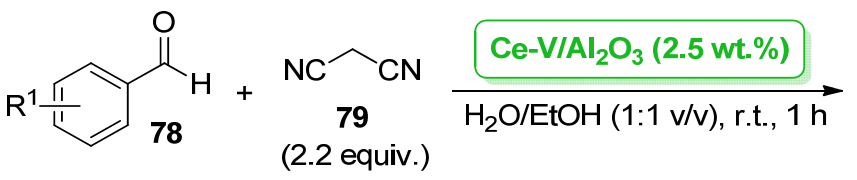<smiles>[R1]c1ccccc1-c1c(C#N)c(N)[n+]([Hg])c(OCC)c1C#N</smiles>

$\mathrm{R}^{1}=\mathrm{H}, 2-\mathrm{OMe}$, 3-OMe, 2-Cl, 2-NO 2 , 2-Br, 4-NMe 2 , 2-Br, 4-F, 4-CF $3(86-94 \%)$

Scheme 23. Synthesis of pyridines 80 by multicomponent reaction of benzaldehydes $\mathbf{7 8}$, malonitrile 79 and ethanol catalyzed by $\mathrm{Ce}-\mathrm{V} / \mathrm{Al}_{2} \mathrm{O}_{3}$, reported in 2016 by Lavanya and co-workers.

The use of iron(III) chloride supported on basic alumina $\left(\mathrm{FeCl}_{3} / \mathrm{Al}_{2} \mathrm{O}_{3}\right)$ was reported in 2017 by Song et al. for the synthesis of functionalized pyrazolo[3,4- $b$ ]pyridines 84 through the multicomponent reaction of 1-heteroaryl-3-phenyl- $1 H$-pyrazol-5-amines 81, benzaldehydes 82 and benzoylacetonitrile 83 in refluxing ethanol (Scheme 24); however, no studies on the reusability and heterogeneity of the $\mathrm{FeCl}_{3} / \mathrm{Al}_{2} \mathrm{O}_{3}$ catalyst were performed [77].

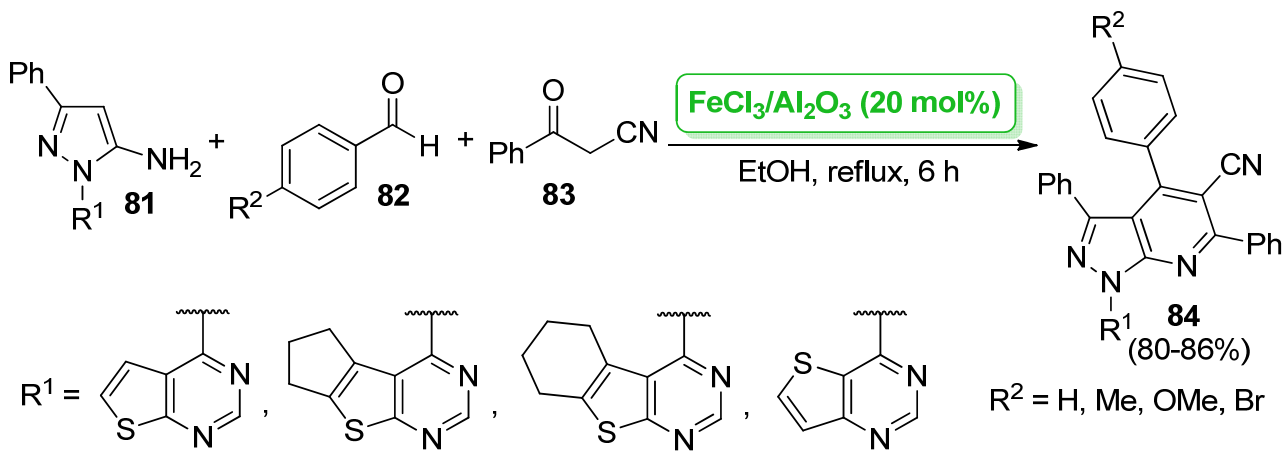

Scheme 24. Synthesis of pyrazolo[3,4- $b$ ]pyridines 84 by multicomponent reaction of pyrazol-5-amines 81, benzaldehydes 82 and benzoylacetonitrile 83 catalyzed by $\mathrm{FeCl}_{3} / \mathrm{Al}_{2} \mathrm{O}_{3}$, reported in 2017 by Song et al.

Few other protocols for the synthesis of $N$-heterocycles based on alumina-supported transition metal catalysts have been reported in recent years: $\mathrm{Pd} / \mathrm{Al}_{2} \mathrm{O}_{3}$ was used in a single example of tandem isomerization/imine formation/ring closing/dehydrogenation of allyl alcohol with $o$-phenylenediamine for the synthesis of 2-ethyl-benzimidazole [78], as well as in the preparation of 2-methylpiperazine by reductive cycloamination of acetol in the presence of ethylenediamine [79]; supported $\mathrm{Pt} / \mathrm{Al}_{2} \mathrm{O}_{3}$ was instead used in combination with $\mathrm{ZnO}$ and a homogeneous acid catalyst ( $p$-toluenesulfonic acid) for the synthesis of indoles and quinolines by acceptorless dehydrogenative condensation of anilines with diols [80].

Titania $\left(\mathrm{TiO}_{2}\right)$ is another metal oxide widely used as a support for metal catalysts, particularly those based on gold species. In the context of the synthesis of $N$-heterocycles, the first study was reported in 2014 by Liu and et al.: they tested the performance of several gold-supported catalysts in the synthesis of benzimidazoles 86 through the reaction of 2-nitroanilines 85 with $\mathrm{CO}_{2}$ in the presence of $\mathrm{H}_{2}$, but commercial $\mathrm{Au} / \mathrm{TiO}_{2}$ gave the best performance [81]. Under the optimized conditions, reactions were performed in NMP as the solvent, using $1 \mathrm{~mol} \%$ of $\mathrm{Au} / \mathrm{TiO}_{2}$ under $\mathrm{H}_{2}(5 \mathrm{MPa})$ and $\mathrm{CO}_{2}(8 \mathrm{MPa})$ atmosphere, at $100{ }^{\circ} \mathrm{C}$ for $12 \mathrm{~h}$ (Scheme 25); unfortunately, the $\mathrm{Au} / \mathrm{TiO}_{2}$ catalyst showed low recyclability, probably due to a marked growth of the mean size of gold nanoparticles observed by TEM after the second run. 


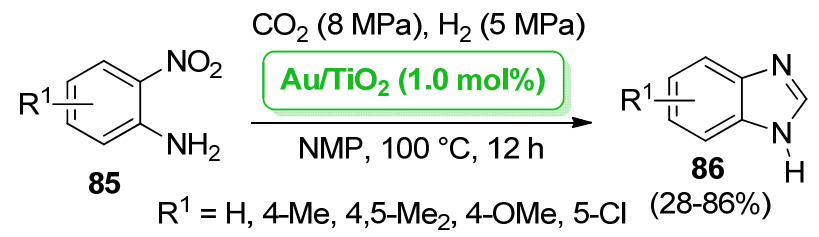

Scheme 25. Synthesis of benzimidazoles 86 by reaction of 2-nitroanilines 85 with $\mathrm{CO}_{2}$ in the presence of $\mathrm{H}_{2}$ catalyzed by commercial $\mathrm{Au} / \mathrm{TiO}_{2}$, reported in 2014 by Liu and et al.

In the same year, Wang and co-workers reported the preparation of gold nanoparticles supported on $\mathrm{TiO}_{2}\left(\mathrm{Au} / \mathrm{TiO}_{2}\right)$ via the deposition-precipitation procedure, which were then used in the synthesis of benzoxazoles 90 and benzimidazoles 91 by two hydrogentransfer reaction of alcohols 87 with, respectively, 2-nitrophenols 88 and 2-nitroanilines 89, performed at $130-150{ }^{\circ} \mathrm{C}$ in toluene as the solvent (Scheme 26) [82]. Interestingly, this catalytic system showed better performance in terms of reusability compared to the abovedescribed commercial $\mathrm{Au} / \mathrm{TiO}_{2}$ tested by Liu and et al.: in the reaction of 2-nitrophenol with benzyl alcohol, the catalyst was used for seven cycles without any reduction in the yield of the corresponding benzoxazole product; however, no hot filtration tests or metal leaching analysis have been performed in order to rule out the occurrence of homogeneous gold species in the solution. In a following publication, the same group extended the use of the same catalyst to the synthesis of 2,4-disubstituted quinazolines by a similar twohydrogen-transfer reaction of primary alcohols with ortho-nitroacetophenones, although performed in the presence of ammonia as a nitrogen source [83].

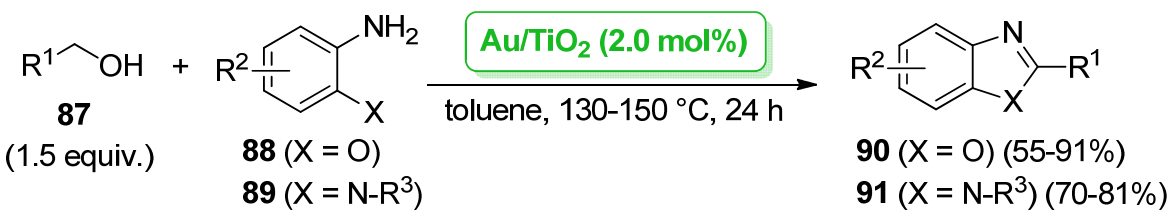

$\mathrm{R}^{1}=\mathrm{Ph}, 4-\mathrm{Me}-\mathrm{C}_{6} \mathrm{H}_{4}, 3-\mathrm{Me}-\mathrm{C}_{6} \mathrm{H}_{4}, 2-\mathrm{Me}_{-} \mathrm{C}_{6} \mathrm{H}_{4}, 4-\mathrm{OMe}-\mathrm{C}_{6} \mathrm{H}_{4}, 3-\mathrm{OMe}-\mathrm{C}_{6} \mathrm{H}_{4}, 2-\mathrm{OMe}-\mathrm{C}_{6} \mathrm{H}_{4}$, 4-F- $\mathrm{C}_{6} \mathrm{H}_{4}, 3-\mathrm{F}-\mathrm{C}_{6} \mathrm{H}_{4}, 4-\mathrm{Cl}-\mathrm{C}_{6} \mathrm{H}_{4}, 4-\mathrm{Br}_{-} \mathrm{C}_{6} \mathrm{H}_{4}, 4-\mathrm{CF}_{3}-\mathrm{C}_{6} \mathrm{H}_{4}$, 1-naphthyl, Me, $t$-Bu, 1-pentyl, Cy $\mathrm{R}^{2}=4-\mathrm{Me}, 5-\mathrm{Me}, 4-\mathrm{OMe}, 5-\mathrm{F}, 4-\mathrm{F}, 4-\mathrm{Cl}$ $\mathrm{R}^{3}=\mathrm{H}, \mathrm{Me}$

Scheme 26. Synthesis of benzoxazoles 90 and benzimidazoles 91 by two hydrogen-transfer reaction of alcohols 87 with, respectively, 2-nitrophenols 88 and 2-nitroanilines 89 catalyzed by $\mathrm{Au} / \mathrm{TiO}_{2}$, reported in 2014 by Wang and co-workers.

Very recently, Lykakis and co-workers described the use of commercial $\mathrm{Au} / \mathrm{TiO}_{2}$ as the best gold-supported catalyst for the synthesis of 2-substituted benzimidazoles through the condensation of ortho-phenylenediamine and aldehydes; in addition to the use of a heterogeneous and reusable catalyst (as confirmed by hot filtration and recyclability tests), the present protocol was developed at room temperature, thus in agreement with Principle 6 (Design for Energy Efficiency) of Green Chemistry [84].

Titania has also been reported as the support for other metal species. In 2014, Zhao et al. described the preparation of $\mathrm{CuCl}_{2}$ supported on nano-TiO $\mathrm{T}_{2}$ powder (named $\mathrm{CuCl}_{2} /$ nano$\mathrm{TiO}_{2}$ ) as a catalyst for the synthesis of imidazo[1,2-a]pyridines 94 through the reaction of 2- aminopyridines 92 and enolizable ketones 93 [85]. The protocol, working on a broad range of substrates, was quite appealing from a sustainability point of view: it required no ligands and additives, air as inexpensive oxidant and ethanol as green solvent (Scheme 27). However, recyclability studies of $\mathrm{CuCl}_{2} /$ nano- $\mathrm{TiO}_{2}$ revealed a significant decrease in product yields after the third run, although it was not attributable to copper leaching into the solution (as verified by a hot filtration test and ICP-AES analysis of the resulting filtrate). 


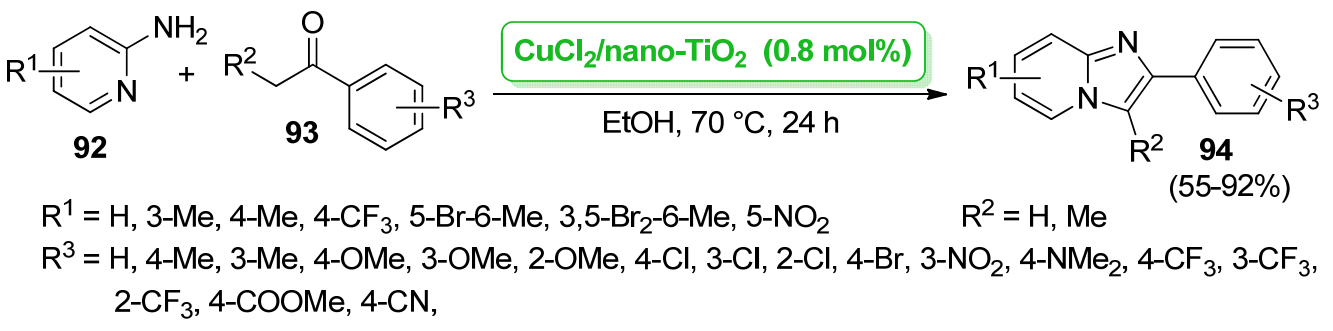

Scheme 27. Synthesis of imidazo[1,2-a]pyridines 94 by reaction of 2-aminopyridines 92 and enolizable ketones 93 catalyzed by $\mathrm{CuCl}_{2} /$ nano- $\mathrm{TiO}_{2}$, reported in 2014 by Zhao et al.

In 2020, Geesi and co-workers described the preparation of copper-doped $\mathrm{TiO}_{2}$ nanoparticles by an ultrasound-assisted sol-gel process from tetrabutyl titanate and copper chloride; the obtained catalyst (known as $\mathrm{Cu}-\mathrm{TiO}_{2}$ ) was then tested in the ultrasoundassisted synthesis of pyridopyrimidines 97 by reaction of 3-(aminomethyl)pyridin-2-amine 95 with benzyl thiols 96 [86]. Although the use of ultrasound as a non-conventional energy source allowed the reactions to be performed at room temperature in short reaction times (90-120 $\mathrm{min})$, the use of $\mathrm{PPh}_{3}$ as a ligand (10 $\mathrm{mol} \%$ ) suggests that the catalytically active species could actually be homogeneous copper complexes leached from the titania support, thus limiting the interest of the protocol in the context of Green Chemistry (Scheme 28).

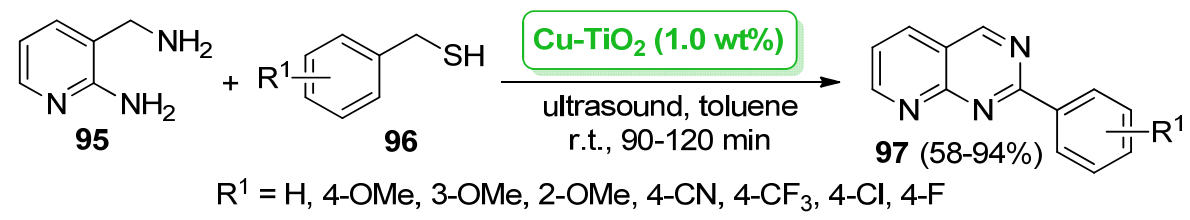

Scheme 28. Synthesis of pyridopyrimidines 97 by reaction of 3-(aminomethyl)pyridin-2-amine 95 with benzyl thiols 96 catalyzed by $\mathrm{Cu}-\mathrm{TiO}_{2}$, reported in 2020 by Geesi and co-workers.

The cascade hydrogenation-cyclocondensation reaction of ortho-dinitrobenzenes 98 with enolizable ketones 99 for the synthesis of 1,5-benzodiazepines 100, performed in the presence of both $\mathrm{Pt} / \mathrm{TiO}_{2}$ and MCM-41 as heterogeneous catalysts, was reported in 2009 by Corma and co-workers; reactions were performed under $\mathrm{H}_{2}$ pressure (7-10 bar) in the absence of a solvent, at $55-100{ }^{\circ} \mathrm{C}$ for $2.5-10 \mathrm{~h}$ (Scheme 29) [87]. In particular, $\mathrm{Pt} / \mathrm{TiO}_{2}$ was used as a catalyst of the starting hydrogenation step, while MCM-41 was used as a Lewis acid catalyst of the following cyclocondensation step. Unfortunately, no recyclability or heterogeneity studies of the $\mathrm{Pt} / \mathrm{TiO}_{2}$ catalyst were reported.

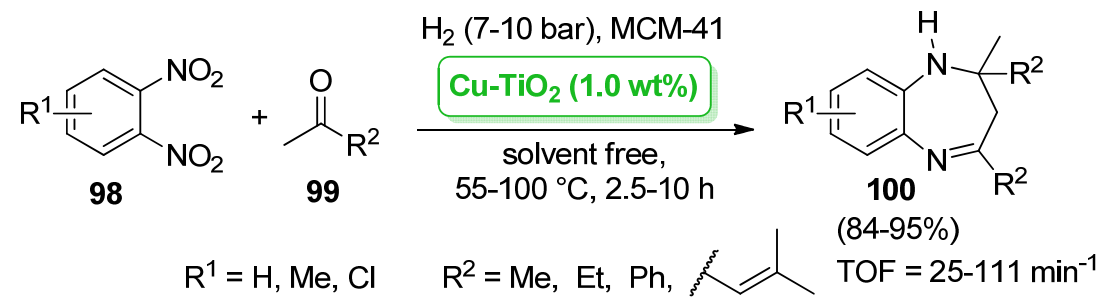

Scheme 29. Synthesis of 1,5-benzodiazepines $\mathbf{1 0 0}$ by cascade hydrogenation-cyclocondensation reaction of ortho-dinitrobenzenes 98 with enolizable ketones 99 catalyzed by $\mathrm{Pt} / \mathrm{TiO}_{2}$ and MCM-41, reported in 2009 by Corma and co-workers.

The same research group more extensively studied synthetic protocols based on the use of metal catalysts supported on ceria $\left(\mathrm{CeO}_{2}\right)$. In particular, in the context of the synthesis of $\mathrm{N}$-heterocycles, they used gold nanoparticles supported on nanoparticulated ceria $\left(\mathrm{Au} / \mathrm{CeO}_{2}\right)$, prepared by mixing a basic solution $(\mathrm{pH}=10)$ of $\mathrm{HAuCl}_{4} \cdot 3 \mathrm{H}_{2} \mathrm{O}$ with a colloidal solution of $\mathrm{CeO}_{2}$ nanoparticles for $18 \mathrm{~h}$ at room temperature, followed by 
a reduction of the resulting solid material with 1-phenylethanol at $160{ }^{\circ} \mathrm{C}$ for $2 \mathrm{~h}$. In the first study, Corma et al. reported the use of $\mathrm{Au} / \mathrm{CeO}_{2}(0.5 \mathrm{~mol} \%)$ in the synthesis of 2-substituted benzimidazoles from primary alcohols and ortho-phenylenediamines by a onepot, four-step protocol, performed at $90{ }^{\circ} \mathrm{C}$ in the presence of $\mathrm{O}_{2}(5 \mathrm{bar})$ as the oxidant and trifluorotoluene as the solvent [88]. The authors pointed out the excellent reusability of the $\mathrm{Au} / \mathrm{CeO}_{2}$ catalyst (together with the absence of metal leaching or deactivation), although the number of cycles has not been specified; moreover, the need for high temperatures and pressures, as well as the use of a toxic solvent, makes the protocol hardly appealing from the point of view of Green Chemistry. In a second investigation, they successfully applied the $\mathrm{Au} / \mathrm{CeO}_{2}$ catalyst in the one-pot two-step synthesis of quinoxalines 103 by oxidative coupling of ortho-phenylenediamines 101 with biomass-derived glycols 102 [89]. Reactions were performed with $1 \mathrm{~mol} \%$ of Au loading, in diglyme as the solvent, at $140{ }^{\circ} \mathrm{C}$ for $24 \mathrm{~h}$, affording quinoxalines 103 in modest to good yields (Scheme 30, path a). Interestingly, in this case the authors performed a detailed study of stability and reusability of the $\mathrm{Au} / \mathrm{CeO}_{2}$ catalyst, which showed a progressive decrease in the product yield after four runs; since the Au content in the recycled catalyst after four runs was the same of that in the freshly prepared sample ( $4.5 \mathrm{wt} \%$, X-ray fluorescence analysis), they excluded the idea that catalyst deactivation was due to gold leaching from the support. Although a progressive increase in the size of $\mathrm{Au}$ nanoparticles due to metal agglomeration was observed by TEM analysis after each run, the authors hypothesized that the organic deposited on the catalyst surface could be the primary cause of its deactivation. In a third, more recent study, Corma et al. extended the abovementioned protocol to the synthesis of 2-(benzoimidazol-2yl)quinoxalines 107: in this case, ortho- phenylenediamines 101 were treated with glycerol 104 under the same experimental conditions $\left(\mathrm{Au} / \mathrm{CeO}_{2} 1 \mathrm{~mol} \%\right.$, diglyme, $\left.140{ }^{\circ} \mathrm{C}, 24 \mathrm{~h}\right)$, thus affording the final product 107 through intermediates 105 or 106 (Scheme 30, path b) [90].

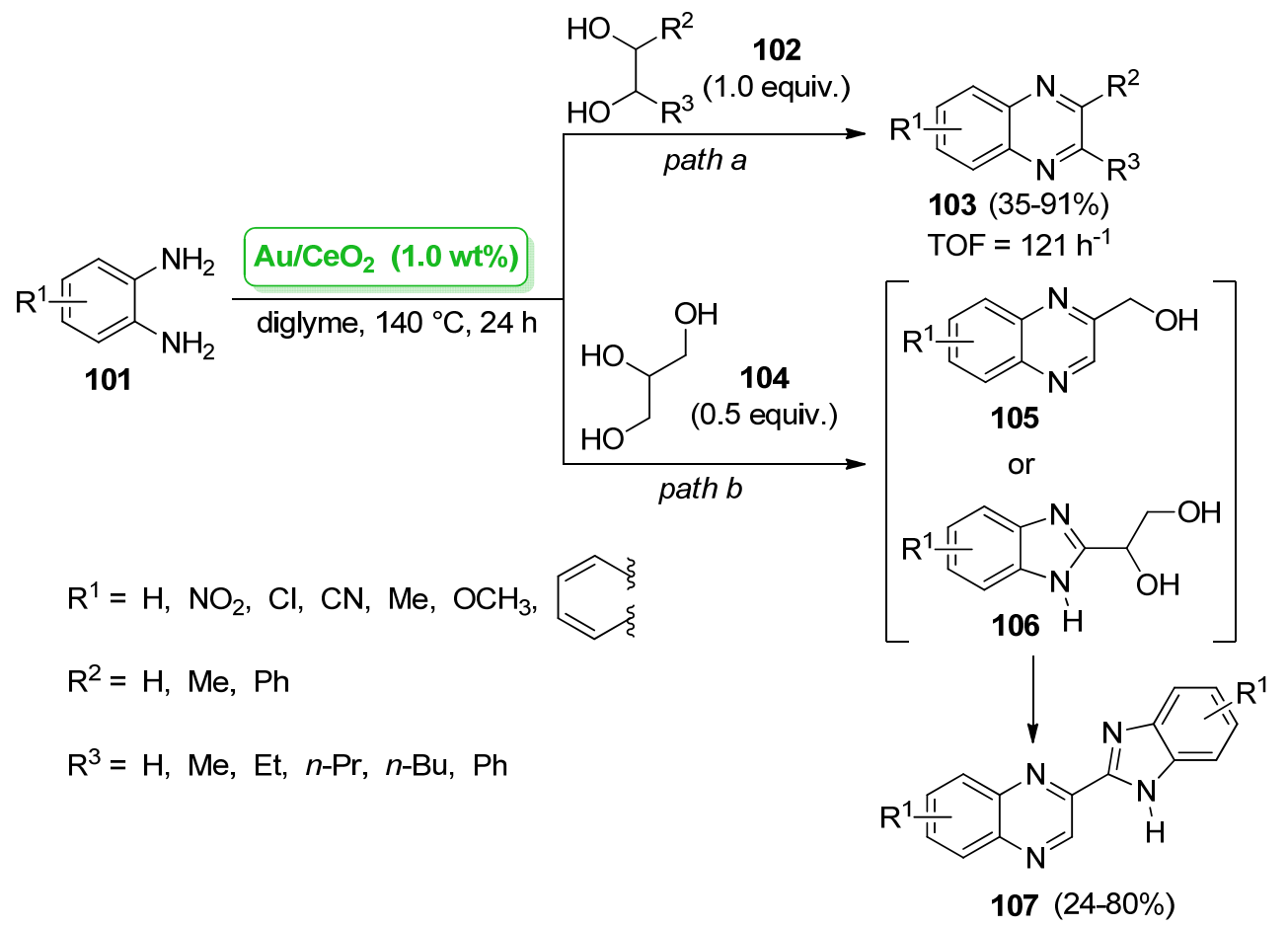

Scheme 30. Synthesis of quinoxalines 103 by oxidative coupling of ortho-phenylenediamines 101 with biomass-derived glycols 102 (path a) and synthesis of 2-(benzoimidazol-2-yl)quinoxalines 107 by oxidative coupling of ortho-phenylenediamines 101 with glycerol 104 (path b) catalyzed by $\mathrm{Au} / \mathrm{CeO}_{2}$, reported in 2012-2013 by Corma and co-workers.

An example of ceria-supported ruthenium catalyst $\left(\mathrm{Ru} / \mathrm{CeO}_{2}\right)$ was reported in 2011 by Wada et al. for the synthesis of indole 109 by intramolecular dehydrogenative hete- 
rocyclization of 2-(2-aminophenyl)ethanol 108 [91]. Although no substrate scope studies were carried out, the authors investigated the synthetic protocol from the catalyst point of view: $\mathrm{Ru} / \mathrm{CeO}_{2}$ was found to be the most effective system in comparison with other similar ruthenium species supported on other inorganic matrices (silica, alumina, titania and magnesia), affording indole in a $99 \%$ yield by the reaction at $140{ }^{\circ} \mathrm{C}$ for $24 \mathrm{~h}$ with $2.5 \mathrm{~mol} \%$ of Ru loading (Scheme 31). Hot filtration tests and ICP-AES analysis of the resulting filtrate showed negligible leaching of $\mathrm{Ru}$ species into the solution, thus indicating that $\mathrm{Ru} / \mathrm{CeO}_{2}$ worked as a merely heterogeneous catalyst. Despite these appealing features, the main limitation of this investigation is represented by the recyclability studies: $\mathrm{Ru} / \mathrm{CeO}_{2}$ was tested only in two consecutive runs, which is too little to evaluate its effective reusability and stability.

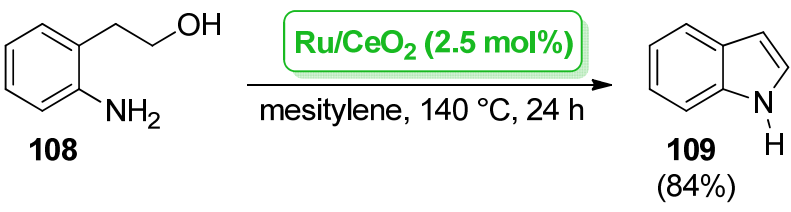

Scheme 31. Synthesis of indole 109 by dehydrogenative heterocyclization of 2-(2-aminophenyl)ethanol 108 catalyzed by $\mathrm{Ru} / \mathrm{CeO}_{2}$, reported in 2011 by Wada et al.

Zirconia is another typical metal oxide used as a support for several metal catalysts. In the context of the synthesis of $N$-heterocyclic compounds, the only examples were reported by Jonnalagadda's research group. In 2015, they described the preparation of a $\mathrm{ZrO}_{2}-$ supported copper oxide catalyst $\left(\mathrm{CuO} / \mathrm{ZrO}_{2}\right)$ by a typical deposition-precipitation method, which was successfully applied in the sustainable synthesis of pyrazole-4-carbonitrile derivatives 113 by a multicomponent reaction of substituted aldehydes $\mathbf{1 1 0}$ with phenylhydrazine 111 and malononitrile 112, performed in water at $40{ }^{\circ} \mathrm{C}$ for $1.5 \mathrm{~h}$ (Scheme 32) [92]. The protocol was of high interest for scientists working in the field of sustainable chemistry, since the use of a non-toxic and inexpensive solvent, the need for modest thermal heating and short reaction times, and the good recyclability of the catalyst (five runs with no decrease in the catalytic activity) fit well with many principles of Green Chemistry. In a following study, they instead explored the use of samarium-doped $\mathrm{ZrO}_{2}\left(\mathrm{Sm}_{2} \mathrm{O}_{3} / \mathrm{ZrO}_{2}\right)$ as a catalyst for the synthesis of 1,4-dihydropyridine derivatives through a similar multicomponent protocol, carried out at room temperature in ethanol as the solvent [93].

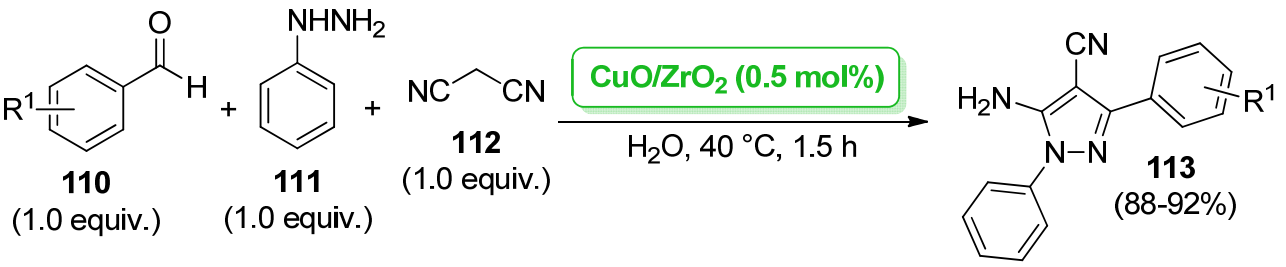

$\mathrm{R}^{1}=\mathrm{H}, 4-\mathrm{Br}, 4-\mathrm{CF}_{3}, 2-\mathrm{Cl}, 4-\mathrm{OH}, 4-\mathrm{OMe}, 2-\mathrm{Br}, 3,4-(\mathrm{OH})_{2}, 2,5-(\mathrm{OH})_{2}, 2,4-(\mathrm{OH})_{2}, 2,3-(\mathrm{OH})_{2}$

Scheme 32. Synthesis of pyrazole-4-carbonitrile derivatives $\mathbf{1 1 3}$ by multicomponent reaction of substituted aldehydes $\mathbf{1 1 0}$ with phenylhydrazine $\mathbf{1 1 1}$ and malononitrile $\mathbf{1 1 2}$ catalyzed by $\mathrm{CuO} / \mathrm{ZrO}_{2}$, reported in 2015 by Jonnalagadda et al.

Other metal oxides (manganese oxide, zinc oxide, niobium oxide) have only occasionally been used as supports of metal catalysts for the synthesis of nitrogen-based heterocycles. In 2015, Veisi et al. reported the synthesis of 2-aryl-1-arylmethyl-1H-1,3-benzimidazoles by the reaction of ortho-phenylenediamines with benzaldehydes ( 2.0 equiv.) under solvent-free conditions at $80^{\circ} \mathrm{C}$, promoted by cobalt-doped manganese oxide nanoparticles, which were tested as an easily recyclable catalyst for four consecutive runs [94]. Copper supported on acid-modified manganese oxide octahedral molecular sieves (named $\mathrm{Cu} / \mathrm{H}-\mathrm{OMS}-2$ ) was 
developed in 2016 by Zhao and co-workers as an efficient catalytic system for the preparation of 3-aroylimidazo[1,2-a]pyri(mi)dines 116 through tandem Michael addition/oxidative cyclization of 2-aminopyri(mi)dines $\mathbf{1 1 4}$ with chalcones $\mathbf{1 1 5}$ [95]. Under optimized conditions, reactions were performed at $100^{\circ} \mathrm{C}$ for $20 \mathrm{~h}$ under air (used as inexpensive sacrificial oxidant), in a tetrachloroethane/acetic acid mixture (1:1 $\mathrm{v} / \mathrm{v})$ as the solvent, which was found to be highly efficient in promoting both Michael addition and oxidative cyclization steps of the protocol (Scheme 33). However, the modest recyclability of $\mathrm{Cu} / \mathrm{H}-\mathrm{OMS}-2$ (as testified by the product yield decrease from the second/third run), together with the use of a chlorinated toxic solvent, makes the protocol unattractive from the point of view of the Twelve Principles of Green Chemistry.

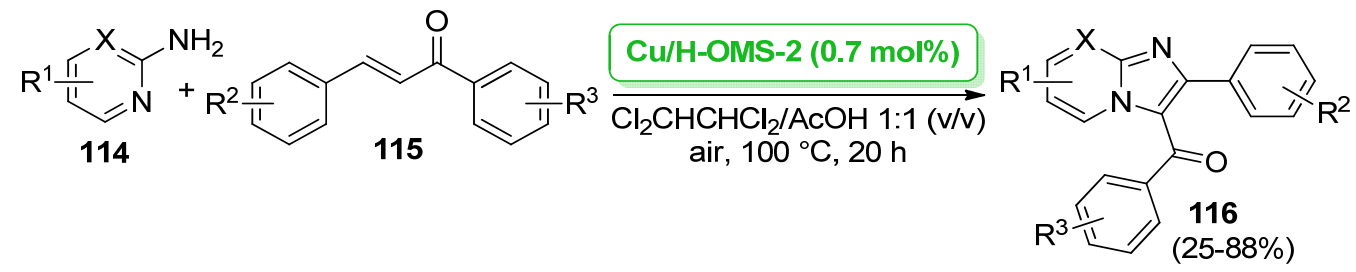

$$
\begin{gathered}
\mathrm{X}=\mathrm{C}, \mathrm{N} \quad \mathrm{R}^{1}=\mathrm{H}, 3-\mathrm{Me}, 4-\mathrm{Me}, 5-\mathrm{Me}, 4-\mathrm{CF}_{3}, 3-\mathrm{Br}, 5-\mathrm{Br}, 4-\mathrm{Cl}, 5-\mathrm{Cl}, 5-\mathrm{COOMe}, 3-\mathrm{CN} \\
\mathrm{R}^{2}=\mathrm{H}, 4-\mathrm{Cl}, 2-\mathrm{Cl}, 4-\mathrm{OMe}, 4-\mathrm{NO} 2,4-\mathrm{F} \quad \mathrm{R}^{3}=\mathrm{H}, 4-\mathrm{Cl}, 4-\mathrm{OMe}, 3,4-(\mathrm{OMe})_{2} \text {, 4-Me }
\end{gathered}
$$

Scheme 33. Synthesis of 3-aroylimidazo[1,2-a]pyri(mi)dines $\mathbf{1 1 6}$ by tandem Michael addition/oxidative cyclization of 2-aminopyri(mi)dines $\mathbf{1 1 4}$ with chalcones $\mathbf{1 1 5}$ catalyzed by $\mathrm{Cu} / \mathrm{H}-\mathrm{OMS}-2$, reported in 2016 by Zhao and co-workers.

A copper oxide supported on a zinc oxide $(\mathrm{CuO} / \mathrm{ZnO})$ nanocatalyst was described in 2015 by Albadi et al. for the synthesis of 1,2,3-1H-triazoles by [3 + 2] cycloaddition of benzyl halides and terminal alkynes in water as the solvent [96]. In the same year, Shimizu and co-workers reported the dehydrogenative $\mathrm{N}$-heterocyclization reaction of 2-(2-aminophenyl)- ethanol to give $1 \mathrm{H}$-indole, performed in refluxing ortho-xylene for $1 \mathrm{~h}$ in the presence of metallic platinum species supported on niobium oxide $\left(\mathrm{Pt} / \mathrm{Nb}_{2} \mathrm{O}_{5}\right)$ [97]. The catalyst, easily synthesized by wet impregnation of $\mathrm{Nb}_{2} \mathrm{O}_{5}$ with an aqueous $\mathrm{HNO}_{3}$ solution of $\mathrm{Pt}\left(\mathrm{NH}_{3}\right)_{2}\left(\mathrm{NO}_{3}\right)_{2}$, followed by drying and reduction under $\mathrm{H}_{2}$ flow, was found to be easily recoverable and recyclable up to three runs with no loss of catalytic activity.

The last class of metal oxides as a support for metal catalysts that we will consider in this section is represented by iron oxides. There are various types of iron oxides, including wüstite $(\mathrm{FeO})$, magnetite $\left(\mathrm{Fe}_{3} \mathrm{O}_{4}\right)$, hematite $\left(\alpha-\mathrm{Fe}_{2} \mathrm{O}_{3}\right)$, iron(III) oxide beta phase $\left(\beta-\mathrm{Fe}_{2} \mathrm{O}_{3}\right)$, maghemite $\left(\gamma-\mathrm{Fe}_{2} \mathrm{O}_{3}\right)$ and iron(III) oxide epsilon phase $\left(\varepsilon-\mathrm{Fe}_{2} \mathrm{O}_{3}\right)$. Most of these materials are used as valuable supports of transition metal catalysts, sometimes also in the form of ferrites $\left(\mathrm{MFe}_{2} \mathrm{O}_{4}\right)$, i.e., ceramic materials made by iron oxides blended with small amounts of one or more additional metals. The most attractive feature of these magnetic systems is their easy separation without the need to utilize filtration or centrifugation techniques, by simply using a magnet.

In the context of the synthesis of $\mathrm{N}$-heterocycles, copper species supported on iron oxides in the form of $\mathrm{CuFe}_{2} \mathrm{O}_{4}$ ferrite have been widely investigated. In 2012, Nageswar et al. reported the use of copper ferrite $\left(\mathrm{CuFe}_{2} \mathrm{O}_{4}\right)$ nanoparticles as a recoverable catalyst for the synthesis of 1,4-disubstituted 1,2,3-1H-triazoles 119 by multicomponent tandem nucleophilic substitution/[3 + 2] cycloaddition of primary bromides 117, terminal alkynes 118 and sodium azide [98]. In particular, reactions were performed in $\mathrm{H}_{2} \mathrm{O}$ at $70{ }^{\circ} \mathrm{C}$ for $4-9 \mathrm{~h}$, in the presence of $5 \mathrm{~mol} \%$ of $\mathrm{CuFe}_{2} \mathrm{O}_{4}$ nanoparticles (Scheme 34). Recyclability studies carried out on four consecutive runs showed a progressive decrease in triazole yields, which was mostly attributed to a decrease in the amount of the recovered catalyst (despite the theoretical advantage to be magnetically recoverable), rather than to metal leaching into the solution. A very similar $\mathrm{CuFe}_{2} \mathrm{O}_{4}$-catalyzed multicomponent protocol for the synthesis of 1,2,3-1H-triazoles, involving boronic acids instead of primary bromides, was developed by Sreedhar and co-workers in 2013 [99]. 


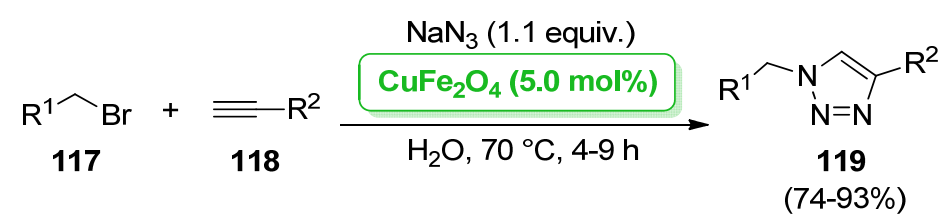

$\mathrm{R}^{1}=\mathrm{Ph}, 4-\mathrm{Br}-\mathrm{C}_{6} \mathrm{H}_{4}, 4-\mathrm{Me}-\mathrm{C}_{6} \mathrm{H}_{4}, 4-\mathrm{NO}_{2}-\mathrm{C}_{6} \mathrm{H}_{4}, \mathrm{COPh}, 2-\mathrm{Br}_{-} \mathrm{C}_{6} \mathrm{H}_{4}, 3-\mathrm{Br}-\mathrm{C}_{6} \mathrm{H}_{4}$ $\mathrm{R}^{2}=\mathrm{Ph}, 4-\mathrm{Me}-\mathrm{C}_{6} \mathrm{H}_{4}, 3-\mathrm{F}-\mathrm{C}_{6} \mathrm{H}_{4}, 3,5-\left(\mathrm{CF}_{3}\right)_{2}-\mathrm{C}_{6} \mathrm{H}_{3}$, 8-OMe-2-naphthyl, 2-pyridyl

Scheme 34. Synthesis of 1,4-disubstituted 1,2,3-triazoles 119 by multicomponent tandem nucleophilic substitution/[3 + 2] cycloaddition of primary bromides 117, terminal alkynes 118 and sodium azide catalyzed by $\mathrm{CuFe}_{2} \mathrm{O}_{4}$, reported in 2012 by Nageswar et al.

$\mathrm{CuFe}_{2} \mathrm{O}_{4}$ nanoparticles were also successfully used as a heterogeneous and magnetically recoverable catalyst in the synthesis of 1,2,4,5-tetrasubstituted $1 \mathrm{H}$-imidazoles $\mathbf{1 2 3}$ by one-pot condensation-cyclization of (hetero)aryl aldehydes 120, 1,2-diketone 121 and propargylamine 122 in the presence of ammonium acetate and under an environmentally friendly ethanol/water (1:3 v/v) solvent mixture in only 30-50 min (Scheme 35) [100]. Recyclability studies of the $\mathrm{CuFe}_{2} \mathrm{O}_{4}$ catalyst showed that it could be used up to six times without any loss of the initial catalytic activity. Moreover, TEM analysis comparison between freshly- prepared and sixth cycle-recovered samples revealed almost the same mean diameter (24 vs. $26 \mathrm{~nm}$ ), confirming a good stability. However, in the present investigation, hot filtration tests and/or metal leaching determinations are missing. In 2014, a three-component synthesis of 2,4,5-trisubstituted imidazoles from aromatic aldehydes, 1,2-diketones and $\mathrm{NH}_{4} \mathrm{OAc}$ in ethanol as the solvent was described by Sanasi et al.: in this case, both copper and cobalt ferrites $\left(\mathrm{CuFe}_{2} \mathrm{O}_{4}\right.$ and $\left.\mathrm{CoFe}_{2} \mathrm{O}_{4}\right)$ were successfully used as magnetically recoverable catalysts [101].

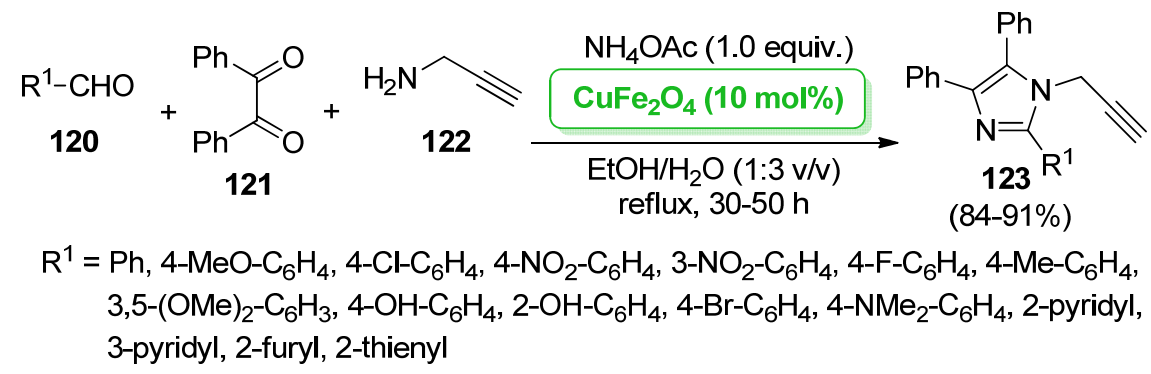

Scheme 35. Synthesis of 1,2,4,5-tetrasubstituted $1 H$-imidazoles 123 by one-pot condensationcyclization of (hetero)aryl aldehydes 120, 1,2-diketone 121 and propargylamine 122 catalyzed by $\mathrm{CuFe}_{2} \mathrm{O}_{4}$, reported in 2015 by El-Remaily et al.

If copper species supported on iron oxides are the most investigated catalytic systems in the context of the preparation of $\mathrm{N}$-heterocycles, other transition metal catalysts have also been occasionally described. In 2009, Tokunaga and co-workers described a one-pot hydrogenation/hydroamination sequence to form indoles $\mathbf{1 2 5}$ from 2-alkynylnitrobenzenes 124, catalyzed by gold nanoparticles supported on $\mathrm{Fe}_{2} \mathrm{O}_{3}\left(\mathrm{Au} / \mathrm{Fe}_{2} \mathrm{O}_{3}\right)$ [102]. Reactions were carried out under $\mathrm{H}_{2}(2.0 \mathrm{MPa})$ as the reducing agent and with a gold loading of $2.3 \mathrm{~mol} \%$, in toluene as the solvent, at $120^{\circ} \mathrm{C}$ for $1 \mathrm{~h}$, affording indoles 125 in good to excellent yields (Scheme 36). Preliminary recycling tests on the $\mathrm{Au} / \mathrm{Fe}_{2} \mathrm{O}_{3}$ catalyst revealed a substantial decrease in its catalytic activity: although a clear aggregation of gold nanoparticles was found by TEM micrographs, the possibility of metal leaching from the support cannot be excluded. 


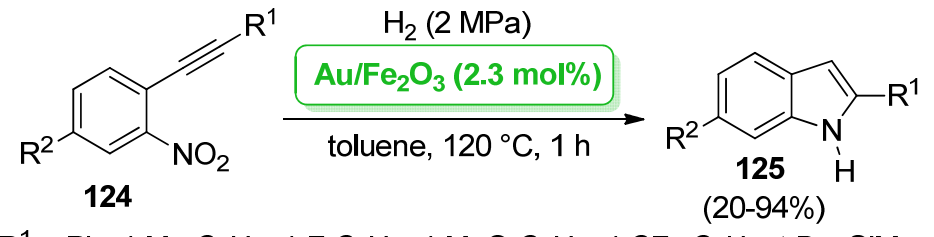

$\mathrm{R}^{1}=\mathrm{Ph}, 4-\mathrm{Me}-\mathrm{C}_{6} \mathrm{H}_{4}, 4-\mathrm{F}-\mathrm{C}_{6} \mathrm{H}_{4}, 4-\mathrm{MeO}-\mathrm{C}_{6} \mathrm{H}_{4}, 4-\mathrm{CF}_{3}-\mathrm{C}_{6} \mathrm{H}_{4}, t-\mathrm{Bu}, \mathrm{SiMe}_{3}$, cyclohex-1-en-1-yl, 1-hexyl

$\mathrm{R}^{2}=\mathrm{H}, \mathrm{Me}$

Scheme 36. Synthesis of indoles 125 by one-pot hydrogenation/hydroamination of 2- alkynylnitrobenzenes 124 catalyzed by $\mathrm{Au} / \mathrm{Fe}_{2} \mathrm{O}_{3}$, reported in 2009 by Tokunaga and co-workers.

A magnetically recoverable nickel catalyst supported on iron oxides, in the form of nickel ferrite $\left(\mathrm{NiFe}_{2} \mathrm{O}_{4}\right)$ nanoparticles, was developed in 2015 by Moghaddam et al. for the one-pot, four-component synthesis of pyrroles from benzaldehydes, 1,3-dicarbonyl compounds, primary amines and nitromethane, at $100{ }^{\circ} \mathrm{C}$ under solvent-free conditions [103]. Although this protocol respects many of the Twelve Principles of Green Chemistry, due to the use of a multicomponent reaction with a high atom economy (Principle 2: Atom Economy), the absence of any solvent (Principle 5: Safer Solvents and Auxiliaries) and the presence of an easily recoverable catalyst (Principle 1: Prevention of Waste; Principle 4: Designing Safer Chemicals; Principle 8: Catalysis), a moderate activity decrease in recycling experiments casts doubts on the possibility of metal contamination into the final products due to the leaching from the support.

A rapid (30 $\mathrm{min}$ ) multicomponent synthesis of 1,4-dihydropyridines 128 from aromatic aldehydes 126, acetoacetates 127 and $\mathrm{NH}_{4} \mathrm{OAc}$ catalyzed by zinc ferrite $\left(\mathrm{ZnFe}_{2} \mathrm{O}_{4}\right)$ nanopowder, in water as the solvent and at room temperature, was described in 2016 by Ravikumar Naik et al. (Scheme 37) [104]. Interestingly, the $\mathrm{ZnFe}_{2} \mathrm{O}_{4}$ catalyst was found to be easily recoverable by simply using a magnet, and recyclable up to five runs with no loss of its catalytic activity, although no studies of metal leaching have been reported.

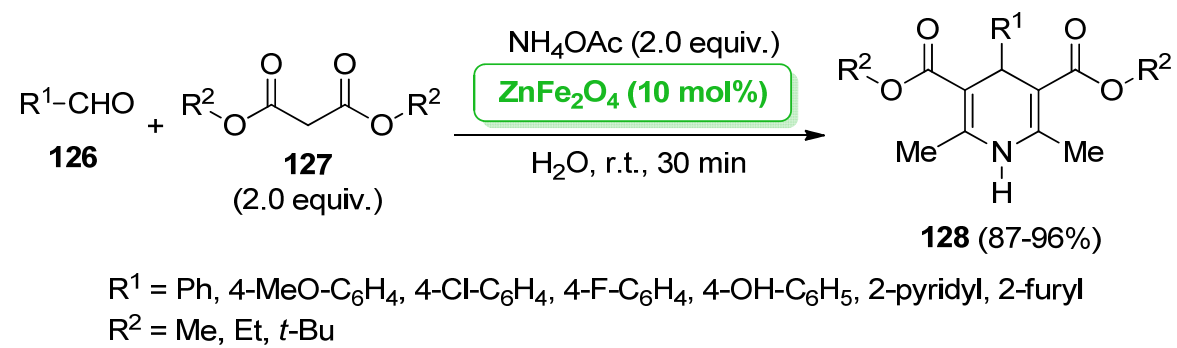

Scheme 37. Multicomponent synthesis of 1,4-dihydropyridines 128 from aldehydes 126, acetoacetates 127 and $\mathrm{NH}_{4} \mathrm{OAc}$ catalyzed by $\mathrm{ZnFe}_{2} \mathrm{O}_{4}$, reported in 2016 by Ravikumar Naik et al.

\subsection{Metal Catalysts Supported on Minerals}

To the best of our knowledge, the first application in the synthesis of $N$-heterocycles of a metal catalyst deposited on minerals as a support was described in 2010 by Wang et al.: copper oxide nanoparticles supported on kaolin (named $\mathrm{CuO} / \mathrm{kaolin}$ ) were used as an efficient catalyst (3.4 mol\% of copper loading) for the preparation of quinazolines 131 through the reaction of 2-aminobenzoketones 129 and benzylic amines 130 (1.5 equiv.), in the presence of tert-butyl hydroperoxide (2.0 equiv.), at $90{ }^{\circ} \mathrm{C}$ for $12 \mathrm{~h}$ under solvent-free conditions (Scheme 38) [105]. Only a modest recyclability of the $\mathrm{CuO} /$ kaolin catalyst was found in the reaction of 2-aminobenzophenone and benzylamine, with a decrease in the product yield from $90 \%$ to $67 \%$ in only four runs, due to copper leaching from the kaolin support (ICP-AES analysis of the mother liquid after catalyst removal). 


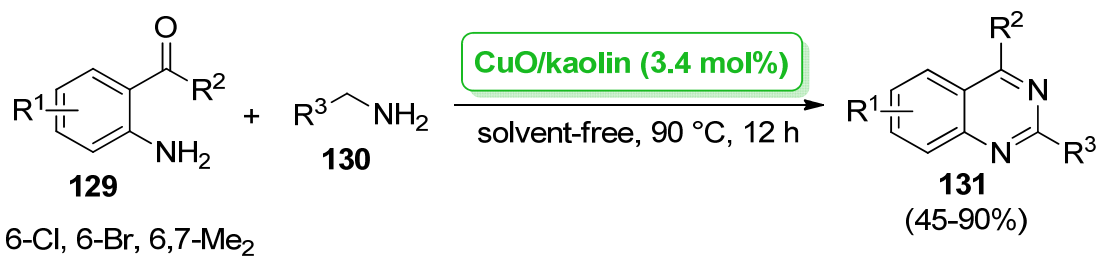

$\mathrm{R}^{1}=6-\mathrm{Cl}, 6-\mathrm{Br}, 6,7-\mathrm{Me}_{2}$

$\mathrm{R}^{2}=\mathrm{Ph}, 4-\mathrm{F}-\mathrm{C}_{6} \mathrm{H}_{4}, 4-\mathrm{Br}-\mathrm{C}_{6} \mathrm{H}_{4}, 4-\mathrm{Me}-\mathrm{C}_{6} \mathrm{H}_{4}, 2,5-\mathrm{Me}_{2}-\mathrm{C}_{6} \mathrm{H}_{3}, 3,5-\mathrm{Me}_{2}-\mathrm{C}_{6} \mathrm{H}_{3}$, Et, $n$-Bu, 1-hexadecyl, $i-\mathrm{Pr}, t-\mathrm{Bu}$, cyclopropyl, cyclopentyl

$\mathrm{R}^{3}=4-\mathrm{Me}-\mathrm{C}_{6} \mathrm{H}_{4}, 3-\mathrm{Me}-\mathrm{C}_{6} \mathrm{H}_{4}, 2-\mathrm{Me}-\mathrm{C}_{6} \mathrm{H}_{4}, 4-\mathrm{MeO}-\mathrm{C}_{6} \mathrm{H}_{4}, 4-\mathrm{Cl}_{-} \mathrm{C}_{6} \mathrm{H}_{4}, 4-\mathrm{F}-\mathrm{C}_{6} \mathrm{H}_{4}, 4-\mathrm{CF}_{3}-\mathrm{C}_{6} \mathrm{H}_{4}$, 1-naphthyl, 2-furyl

Scheme 38. Synthesis of quinazolines $\mathbf{1 3 1}$ by reaction of 2-aminobenzoketones $\mathbf{1 2 9}$ and benzylic amines $\mathbf{1 3 0}$ catalyzed by $\mathrm{CuO} /$ kaolin, reported in 2010 by Wang et al.

More recently, several other minerals have been successfully tested as a support for catalytic copper species. In 2012, Vishwakarma, Bharate and co-workers reported the preparation of a montmorillonite-supported copper(II) catalyst through the treatment of montmorillonite KSF clay with an aqueous $\mathrm{CuSO}_{4} \cdot 5 \mathrm{H}_{2} \mathrm{O}$ solution, followed by solvent evaporation, drying and calcination at $425^{\circ} \mathrm{C}$ for $3 \mathrm{~h}$ [106]. Such a catalyst was then used $(10 \mathrm{~mol} \%)$ in the tandem azidonation/[3 + 2] cycloaddition of aryl boronic acids with $\mathrm{NaN}_{3}$ and terminal alkynes to give $1 H$-1,2,3-triazoles, performed in water at room temperature. The protocol seems quite appealing from the point of view of Green Chemistry, as further testified by the good catalyst recyclability over several cycles without a loss of activity. However, a more appealing protocol from the synthetic point of view, since it was carried out on more functionalized reagents, was described in 2015 by Kumar et al.: a family of alloxan-derived bis-1,2,3- $1 H$-triazoles 134 was synthesized by [3 +2 ] cycloaddition of bis-propargyl alloxan 132 with benzyl azides 133, catalyzed by the same montmorillonitesupported copper(II) catalyst (indicated clay-Cu(II) by the authors) [107]. After a detailed study of the reaction conditions, the substrate scope was investigated in methanol as the best solvent, at room temperature for $4 \mathrm{~h}$, giving final products $\mathbf{1 3 4}$ in almost quantitative yields (Scheme 39). However, in this work, the recyclability of the clay-Cu(II) catalyst appeared slightly lower than that reported in the previous investigation of Vishwakarma, Bharate and co-workers, with a $\sim 20 \%$ decrease in the product yields after five runs. A bimetallic copper-aluminum system supported on hydrotalcite (named CuAl-HT) was developed in 2015 by Li et al. for the synthesis of $1 H$-indoles by intramolecular dehydrogenative $\mathrm{N}$-heterocyclization of 2-(2-aminophenyl)ethanols, performed in the presence of 1.0 equiv. of potassium $t$-butoxide and ortho-xylene as the solvent [108].

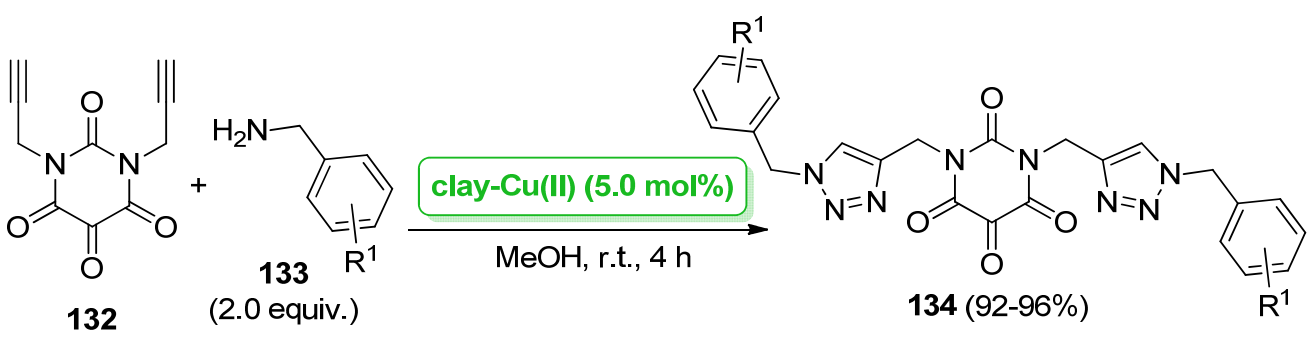

$\mathrm{R}^{1}=\mathrm{H}, 4-\mathrm{OH}, 2-\mathrm{Me}, 4-\mathrm{Cl}$, 4-OMe, 2-OH, 4-Me, 4- $\mathrm{CH}_{2} \mathrm{CH}_{2} \mathrm{OH}, 4-\mathrm{Br}, 4-\mathrm{NO}_{2}$, 2-Cl, 2- $\mathrm{Br}, 2-\mathrm{OMe}$ $4-\mathrm{CH}_{2} \mathrm{CH}_{2} \mathrm{OMe}$

Scheme 39. Synthesis of alloxan-derived bis-1,2,3-1H-triazoles 134 by [3 + 2] cycloaddition of bis- propargyl alloxan 132 with benzyl azides 133 catalyzed by clay-Cu(II), reported in 2015 by Kumar et al.

Hydrotalcite is definitely one of the most common minerals used as a support for metal catalysts. In addition to the above-mentioned investigation of $\mathrm{Li}$ et al. focusing on $\mathrm{CuAl-HT}$, Jonnalagadda and co-workers in 2014 reported the development of a bimetallic 
zinc-vanadium catalyst supported on hydrotalcite (indicated in the paper as $\mathrm{Zn}-\mathrm{VCO}_{3} \mathrm{HT}$ ), prepared by the co-precipitation of zinc nitrate and ammonium metavanadate into a basic solution ( $\mathrm{pH} 10-11$ ) of $\mathrm{Na}_{2} \mathrm{CO}_{3}$ [109]. Such material was tested as an efficient and recoverable catalyst in the Hantzsch reaction of aromatic aldehydes $\mathbf{1 3 5}$ with acetoacetanilide 136 for the preparation of dihydropyridines 137 , carried out in water as the solvent, at $60{ }^{\circ} \mathrm{C}$ for $2 \mathrm{~h}$ (Scheme 40). The good sustainability of the protocol was further confirmed by the very excellent catalyst recyclability: $\mathrm{Zn}-\mathrm{VCO}_{3} \mathrm{HT}$ was used for five runs showing the same activity (product yields ranging between $90 \%$ and $93 \%$ ). However, an important limitation of this work is represented by the lack of studies for the determination of both metals loading on the support. Corma et al. described some representative examples of the synthesis of quinoxalines by oxidative coupling of ortho-phenylenediamines with biomass-derived glycols catalyzed by gold nanoparticles supported on hydrotalcite (Au/HT), used as a possible alternative to $\mathrm{Au} / \mathrm{CeO}_{2}$ (see Scheme 30, path a on Section 2.3), performed under air as an inexpensive oxidant at $140{ }^{\circ} \mathrm{C}$ and in diglyme as the solvent [89].

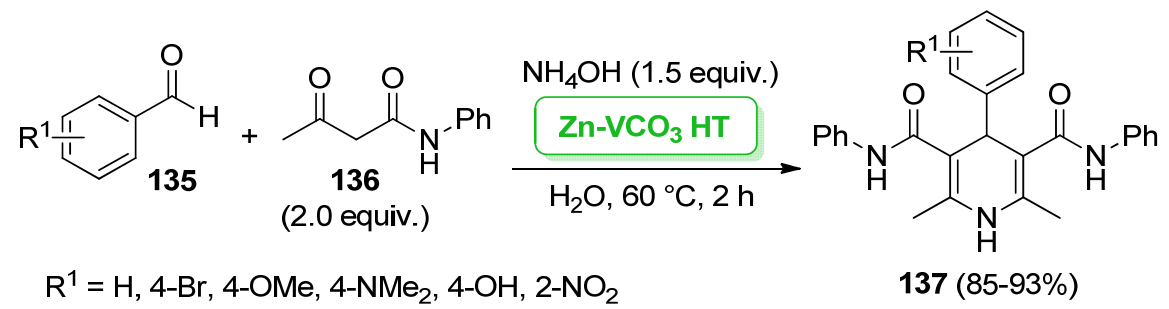

Scheme 40. Synthesis of dihydropyridines 137 by Hantzsch reaction of aromatic aldehydes 135 with acetoacetanilide 136 catalyzed by $\mathrm{Zn}-\mathrm{VCO}_{3} \mathrm{HT}$, reported in 2014 by Jonnalagadda and co-workers.

Zeolites received large attention as a support for metal catalysts, thanks to the possibility of easy tuning of their chemical and physical properties (in particular, their acidity and shape selectivity). In this context, a first preliminary study was reported in 2015 by Shimizu and co-workers: although they described the dehydrogenative $N$-heterocyclization reaction of 2-(2-aminophenyl)ethanol to give $1 \mathrm{H}$-indole catalyzed by $\mathrm{Pt} / \mathrm{Nb}_{2} \mathrm{O}_{5}$, they also reported the same reaction in the presence of platinum supported on HBEA zeolite (named Pt/HBEA), in refluxing ortho-xylene for $1 \mathrm{~h}$ [97]. A more appealing investigation was reported in 2017 by Hong and co-workers, where a Pd-zeolite catalyst was successfully used for the synthesis of azaindoles 140 [110]. The supported catalytic system was prepared through the treatment of commercial $\mathrm{NaY}$ zeolite with a $0.1 \mathrm{M}$ aqueous solution of $\left[\mathrm{Pd}\left(\mathrm{NH}_{3}\right)_{4}\right] \mathrm{Cl}_{2}$ for $24 \mathrm{~h}$ at room temperature, which afforded the ion-exchanged $\left[\mathrm{Pd}\left(\mathrm{NH}_{3}\right)_{4}\right]^{2+}-\mathrm{NaY}$ zeolite, followed by calcination at $500{ }^{\circ} \mathrm{C}$ for $4 \mathrm{~h}$ under an $\mathrm{O}_{2}$ atmosphere and a final reduction with $\mathrm{H}_{2}$ at $300{ }^{\circ} \mathrm{C}$ for $4 \mathrm{~h}$ to give the final $\mathrm{Pd}(0)-\mathrm{NaY}$. The supported palladium catalyst was applied in the heteroannulation of various orthoiodoaminopyridines 138 with acetylenes 139, performed for $12 \mathrm{~h}$ at $140{ }^{\circ} \mathrm{C}$ in DMF with $5 \mathrm{~mol} \%$ palladium in the presence of $\mathrm{LiCl}$ (1.0 equiv.) as an additive and $\mathrm{Cs}_{2} \mathrm{CO}_{3}$ (2.0 equiv.) as the base, to give pyrrolo-pyridines $\mathbf{1 4 0}$ in high yields (Scheme 41). The $\mathrm{Pd}(0)-\mathrm{NaY}$ catalyst showed only a moderate reusability, with a gradual decrease in the catalytic activity after four runs.

Very recently, the use of perlite as a catalyst support has also been reported. Amutha et al. described in 2019 the preparation of a perlite-supported bismuth chloride (indicated as $\mathrm{BiCl}_{3}$-perlite) system by a simple dispersion method, which was then successfully applied as a recoverable Lewis acid catalyst in the condensation of ortho-phenylenediamines 141 with benzyl 142 for the synthesis of quinoxalines 143 [111]. The synthetic protocol complies well with most of the Twelve Principles of Green Chemistry: reactions were carried out at room temperature and in ethanol as a sustainable solvent, in very short times (5-50 min) using $5 \mathrm{wt}$. $\%$ of $\mathrm{BiCl}_{3}$-perlite as a recoverable and highly recyclable (five runs with no loss of activity) catalyst (Scheme 42). 


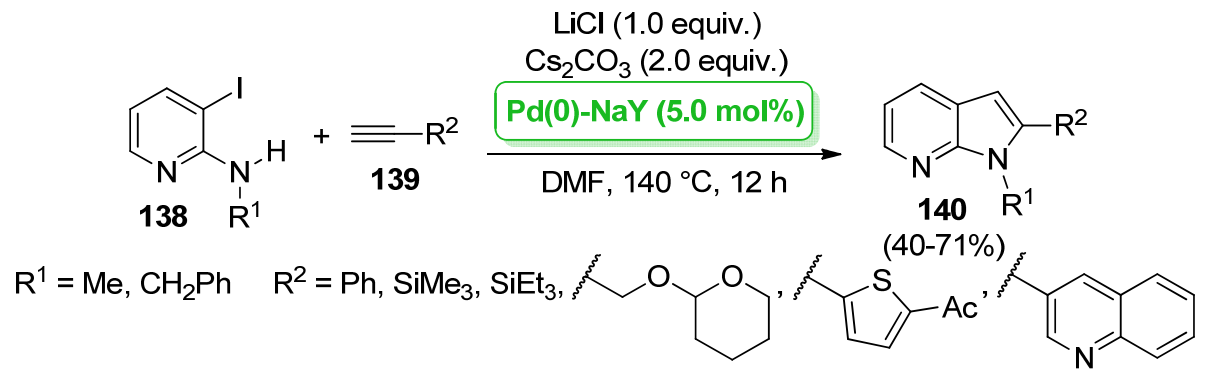

Scheme 41. Synthesis of pyrrolo-pyridines 140 by heteroannulation of ortho-iodoaminopyridines 138 with acetylenes 139 catalyzed by $\mathrm{Pd}(0)-\mathrm{NaY}$, reported in 2017 by Hong and co-workers.

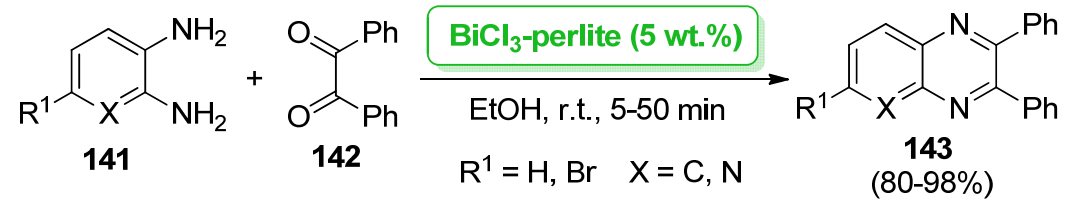

Scheme 42. Synthesis of quinoxalines 143 by condensation of ortho-phenylenediamines 141 with benzyl 142 catalyzed by $\mathrm{BiCl}_{3}$-perlite, reported in 2019 by Amutha et al.

\subsection{Metal Catalysts Supported on Magnetic Core/Inorganic Shell Composites}

During the last decade, magnetic nanomaterials based on iron oxides have acquired large interest in the context of supported catalytic systems, because of their simple preparation, modification and easy separation without the need of filtration or centrifugation techniques, by simply using a magnet. As we described in Section 2.3, such systems are efficiently used as conventional supports for metal catalysts. However, a more recent and appealing alternative is represented by their application in core/shell nanocomposites. These structures, consisting of a magnetic core of iron oxides coated with a shell of a suitable inorganic (or organic) material, are emerging as new challenging supports for metal catalysts, able to combine physico-chemical properties of both constituent materials: the easy recoverability of the magnetic core on one hand, and compatibility with the metal catalyst of the shell on the other. In particular, in this section, we will consider the application of metal catalysts supported on magnetic core/inorganic shell composites for the synthesis of $N$-heterocycles.

Among all the inorganic shells investigated for the preparation of these catalytic composites, the most common are definitely the carbon-based ones, such as graphene oxide and carbon nanotubes. In 2014, Xiong and co-workers reported the preparation of copper bromide supported on core/shell superparamagnetic graphene oxide $\left(\mathrm{CuBr} / \mathrm{GO} @ \mathrm{Fe}_{3} \mathrm{O}_{4}\right)$ via a simple chemical method and its application for the synthesis of 1,2,3-1H-triazoles 147-148 by the $\mathrm{Cu}(\mathrm{I})$-catalyzed azide-alkyne 1,3-dipolar cycloaddition ( $\mathrm{CuAAC}$ ) reaction on a multi-gram scale [112]. Reactions were successfully performed by the treatment of aryl/alkyl halides 144, $\mathrm{NaN}_{3}$ and alkynes 145 or bis-alkynes 146 in water as the solvent, in the presence of $5.0 \mathrm{~mol} \%$ of the supported copper catalyst and under microwave irradiation (Scheme 43). More interestingly, $\mathrm{CuBr} / \mathrm{GO} @ \mathrm{Fe}_{3} \mathrm{O}_{4}$ can be separated from the reaction mixtures using an external permanent magnet and then reused up to six consecutive runs with no noticeable drop in the 1,2,3-1H-triazole yield or its catalytic activity. 


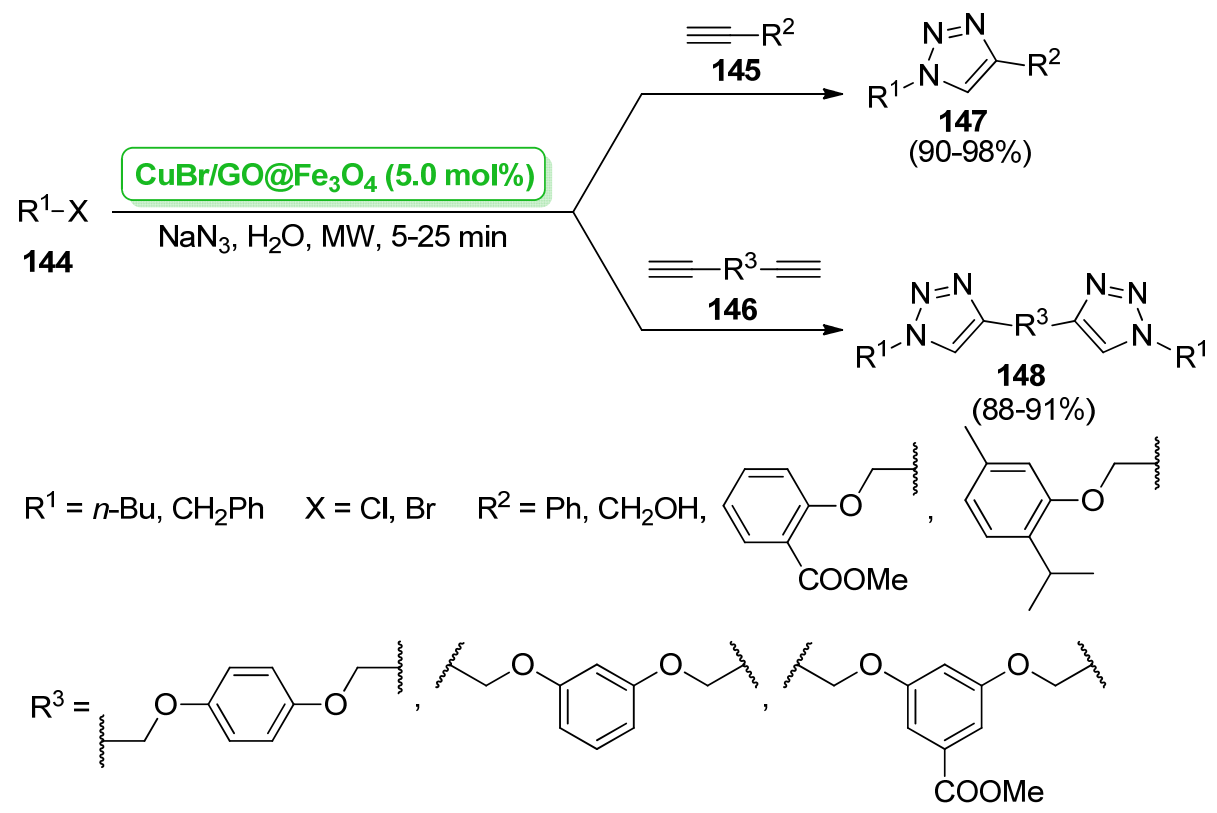

Scheme 43. Synthesis of 1,2,3-1H-triazoles 147-148 by CuAAC reaction of aryl/alkyl halides 144, $\mathrm{NaN}_{3}$ and alkynes 145 or bis-alkynes 146 catalyzed by $\mathrm{CuBr} / \mathrm{GO} @ \mathrm{Fe}_{3} \mathrm{O}_{4}$, reported in 2014 by Xiong et al.

In the same year, Jain et al. described the application of a palladium supported on iron nanoparticles-graphene oxide core/shell nanocomposite (Pd(II)/GO@Fe-FeO) as a heterogeneous catalyst (10 mol\%) for the synthesis of 2-alkylquinolines 151 through the one-pot reaction of anilines 149 with vinyl or allyl ethers 150 (3.0 equiv.), performed in acetonitrile at $80^{\circ} \mathrm{C}$ for $8-12 \mathrm{~h}$ (Scheme 44) [113]. Pd(II)/GO@Fe-FeO also showed high recyclability, since its catalytic activity remains unaltered even after six runs.

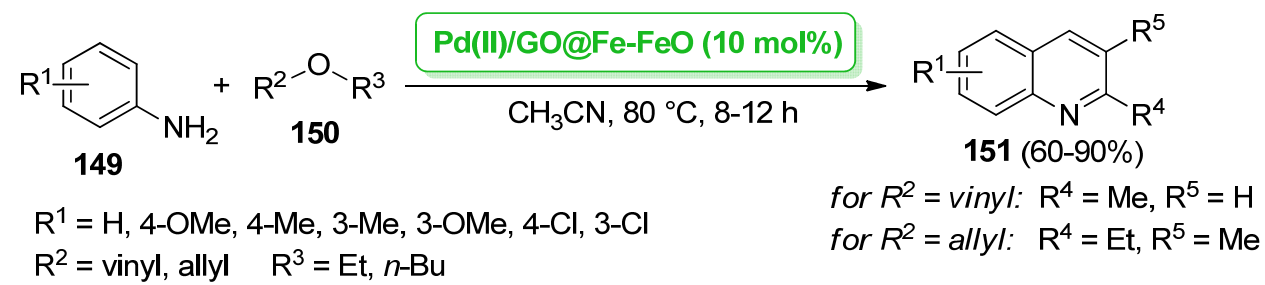

Scheme 44. Synthesis of 2-alkylquinolines 151 by one-pot reaction of anilines 149 with vinyl/allyl ethers 150 catalyzed by Pd(II)/GO@Fe-FeO, reported in 2014 by Jain et al.

A copper catalyst supported on carbon nanotubes grafted on cobalt ferrite nanoparticles (indicated below as $\mathrm{Cu} / \mathrm{CNT} @ \mathrm{CoFe}_{2} \mathrm{O}_{4}$ ) was prepared in 2016 by Zhang and coworkers in a three-step process, consisting of a) the oxidation of carbon nanotubes with $\mathrm{HNO}_{3}$ at $80^{\circ} \mathrm{C}$ for $2 \mathrm{~h}$ under ultrasound, in order to increase the $-\mathrm{OH}$ functional groups on the surface; b) synthesis of the magnetic core/inorganic shell CNT@CoFe $\mathrm{O}_{4}$ nanocomposite, by co-precipitation of $\mathrm{FeCl}_{3}$ and $\mathrm{CoCl}_{2}$ within the carbon nanotubes under refluxing basic conditions; and c) treatment with $\mathrm{CuCl}$ in water at room temperature, giving the final Cu/CNT@CoFe $\mathrm{O}_{4}$ system (Scheme 45a) [114]. Such a catalyst was then applied to the synthesis of imidazo[1,2-a]pyridines 154 by a one-pot multicomponent reaction of 2-aminopyridines 152, benzaldehydes 153 and nitromethane, performed in PEG-400 as the solvent at $80{ }^{\circ} \mathrm{C}$ for $3-6 \mathrm{~h}$ (Scheme $45 \mathrm{~b}$ ). 
(a)
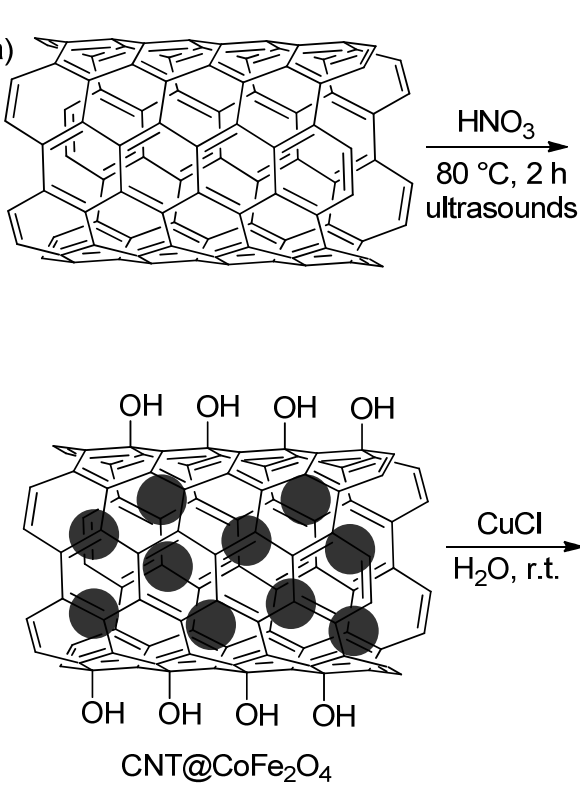

(b)

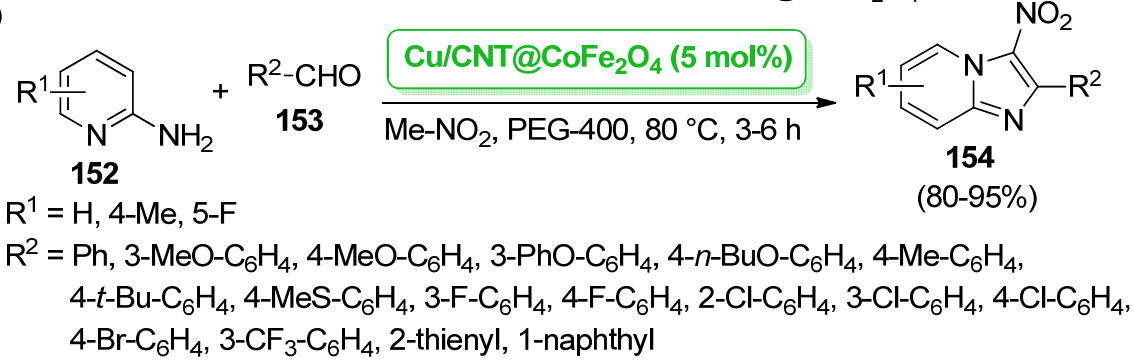

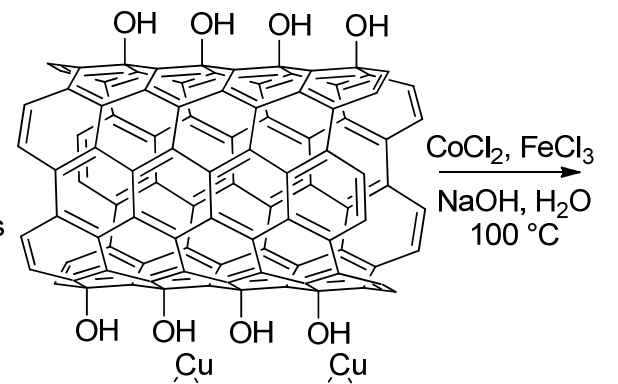

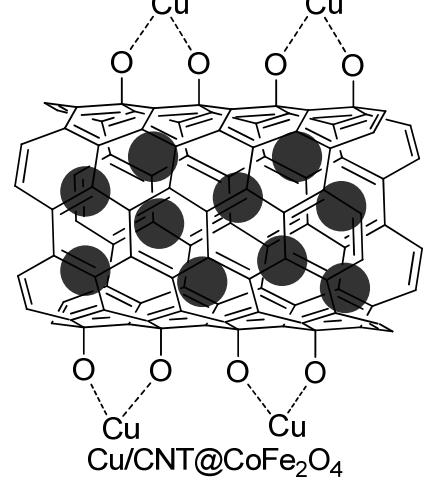

Scheme 45. (a) Schematic route for the preparation of $\mathrm{Cu} / \mathrm{CNT} @ \mathrm{CoFe}_{2} \mathrm{O}_{4}$ catalyst. (b) Synthesis of imidazo[1,2-a]pyridines 154 by multicomponent reaction of 2-aminopyridines 152, benzaldehydes 153 and nitromethane catalyzed by $\mathrm{Cu} / \mathrm{CNT} @ \mathrm{CoFe}_{2} \mathrm{O}_{4}$, reported in 2016 by Zhang and co-workers.

More recently, Gravel, Doris and co-workers reported the development of a new nanohybrid catalyst, consisting of copper nanoparticles on magnetic carbon nanotubes, applied for the one-pot azide formation/1,3-dipolar cycloaddition of organic bromides and chlorides with terminal alkynes, carried out at room temperature for $24 \mathrm{~h}$ in a $\mathrm{H}_{2} \mathrm{O} / \mathrm{EtOH}$ $(4: 1 \mathrm{v} / \mathrm{v})$ solvent mixture [115]. The protocol seems very convenient from the point of view of Green Chemistry, as further confirmed by good recyclability (four runs with only a negligible decrease in the 1,2,3-1H-triazole products yields), suggesting a merely heterogeneous mechanism.

Other inorganic shells have been significantly less used for the preparation of metal catalysts supported on magnetic core/shell composites. In particular, in the context of the preparation of nitrogen-based heterocycles, very few works have been reported, and only very recently. In 2020, Wang et al. described the preparation of a cerium(III) chloride catalyst supported on silica-coated magnetite nanoparticles (indicated as $\mathrm{Fe}_{3} \mathrm{O}_{4} @ \mathrm{SiO}_{2}-\mathrm{CeCl}_{3}$ ) by a rather simple, cost-effective and environmentally friendly procedure (Scheme 46); such material was then applied as a recoverable catalyst in a one-pot, three-component protocol and in a domino process for the synthesis of 3-substituted-1,5-benzodiazepines [116]. In the same year, Maleki and co-workers instead reported the preparation of a nickel catalyst supported on magnetic bentonite clay, obtained by introducing ferrite nanoparticles inside the porous structure of the mineral, which was then tested in the three-component condensation reaction of aldehydes, benzyl and ammonium acetate for the synthesis of imidazoles, performed at $80^{\circ} \mathrm{C}$ in ethanol as the solvent and under ultrasound sonication [117]. 


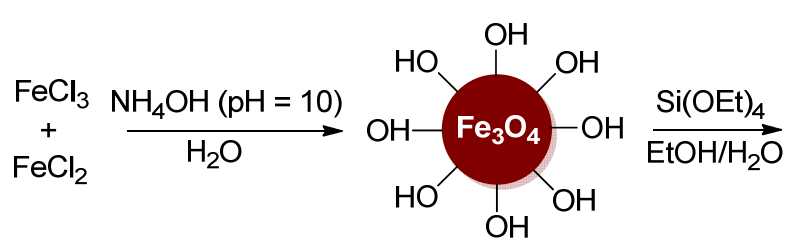

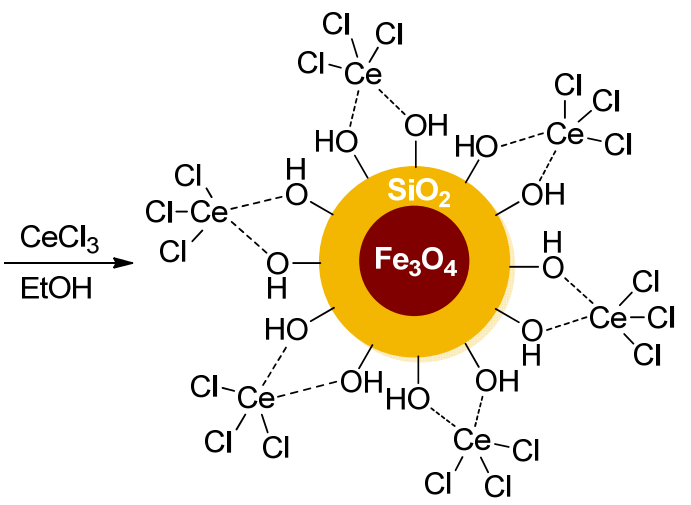

$\mathrm{Fe}_{3} \mathrm{O}_{4} @ \mathrm{SiO}_{2}-\mathrm{CeCl}_{3}$

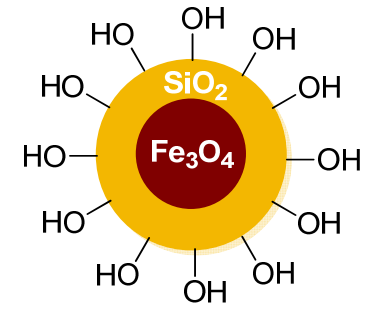

$\mathrm{Fe}_{3} \mathrm{O}_{4} @ \mathrm{SiO}_{2}$

Scheme 46. Schematic route for the preparation of $\mathrm{Fe}_{3} \mathrm{O}_{4} @ \mathrm{SiO}_{2}-\mathrm{CeCl}_{3}$ catalyst developed in 2020 by Wang et al.

\section{Metal Catalysts Supported on Organic Matrices for the Synthesis of $N$-Heterocycles}

In the context of the development of supports for reusable metal catalysts, organic matrices represent a more recent and appealing alternative to inorganic materials. On the one hand, synthetic organic matrices (that is, polymers and polymer-anchored ligands) can be easily obtained with the desired chemical structure and morphology; on the other, natural organic matrices (that is, biopolymers) are commercially available at a low cost and offer the possibility of an easy chemical modification. Moreover, a further benefit of organic supports is that they tend to give less interference in the whole catalytic process with respect to inorganic matrices [118].

In this second part of the manuscript, we will give an overview of the synthetic protocols for the preparation of $N$-heterocycles involving the use of metal catalysts supported on organic matrices; we will start from synthetic organic polymers, consisting of cross-linked polymeric matrices insoluble in the reaction medium (in the present review, we will not consider catalysts supported on soluble matrices, as stated in the introduction); then, we will consider polymer-anchored ligands, which offer the unprecedented opportunity for immobilization and heterogenization of homogeneous complexes; finally, we will take into account natural biopolymers, whose use perfectly fit with the Twelve Principles of Green Chemistry, as they are typically non-toxic, cheap and environmentally friendly.

\subsection{Metal Catalysts Supported on Organic Polymers}

The first investigation focused on the synthesis of $N$-heterocycles promoted by a metal catalyst supported on a synthetic organic polymer was reported in 2005 by Adharvana Chari and co-workers: poly(4-vinylpyridine)-supported ferric chloride $\left(\mathrm{FeCl}_{3} / \mathrm{PVP}\right)$ was used as an inexpensive and eco-friendly catalyst for the synthesis of 1,5-benzodiazepine derivatives 157 through a condensation reaction of ortho-phenylenediamines 155 and enolizable ketones 156, performed in solvent-free conditions under conventional thermal heating (reflux, 30-65 $\mathrm{min}$ ) or microwave irradiation (450 W, 30-90 s) (Scheme 47) [119]. The authors strongly emphasized that their protocol was waste-free (with the generation of $\mathrm{H}_{2} \mathrm{O}$ as the only by-product) and allowed for moderate catalyst recycling (product yields decreased by $\sim 10 \%$ after four runs). 


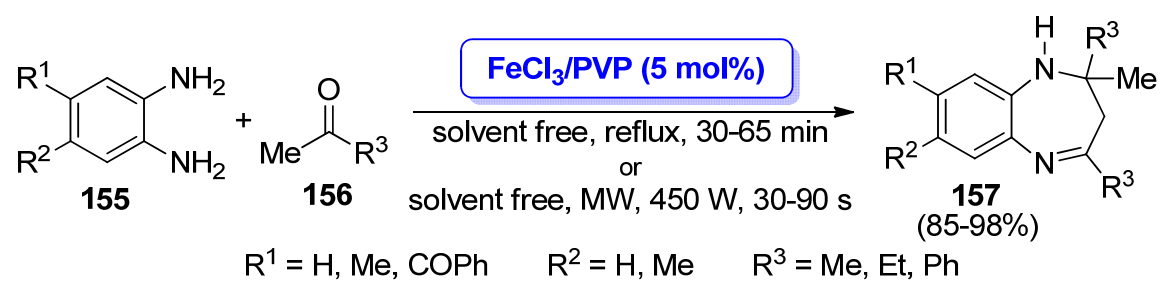

Scheme 47. Synthesis of 1,5-benzodiazepines 157 by condensation of ortho-phenylenediamines 155 and enolizable ketones 156 catalyzed by $\mathrm{FeCl}_{3}$ /PVP, reported in 2005 by Adharvana Chari and co-worker.

Polystyrene-based materials are typical organic matrices used as a support for metal catalysts. In 2014, Heravi et al. described the in situ preparation of copper iodide nanoparticles supported on modified poly(styrene-co-maleic anhydride), which was called SMI-Cu(I) by the authors, used as an efficient and recyclable catalyst for the synthesis of 1,2,3-1H-triazoles from $\alpha$-bromoketones, $\mathrm{NaN}_{3}$ and terminal alkynes by tandem azide formation/[3 +2$]$ cycloaddition reaction, carried out for 20-65 min under refluxing $\mathrm{H}_{2} \mathrm{O}$ [120]. A very interesting study was reported in 2016 by Das and co-workers: palladium nanoparticles supported on polystyrene (indicated as Pd@PS) were easily prepared through the treatment of commercially available Amberlite IRA 900 polystyrene resin with $\mathrm{NaBH}_{4}$ in water at room temperature for $4 \mathrm{~h}$, followed by the reaction of the resulting borohydride exchanged resin beads with $\mathrm{Pd}(\mathrm{OAc})_{2}$ in $\mathrm{DMF}$ at $100{ }^{\circ} \mathrm{C}$ for $1 \mathrm{~h}$ [121]. The supported Pd@PS catalyst was used in the domino decarboxylative coupling/5-exo-dig cyclization of alkynyl carboxylic acids 158 with ortho- iodoanilines 159 for the preparation of indoles 160; the reactions were carried out using $3 \mathrm{~mol} \%$ of Pd@PS in the presence of DBU (3.0 equiv.) as the base, in DMF at $110{ }^{\circ} \mathrm{C}$ for $12 \mathrm{~h}$ (Scheme 48). The same protocol was also applied to amino benzocycloheptene bromides for the preparation of tricyclic 3-pyrroline derivatives.

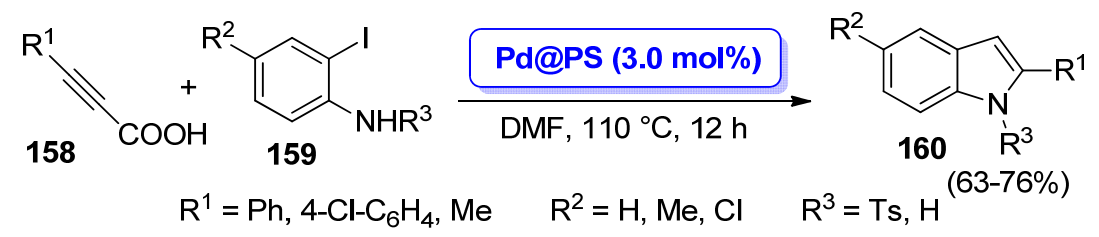

Scheme 48. Synthesis of indoles 160 by domino decarboxylative coupling/5-exo-dig cyclization of alkynyl carboxylic acids 158 with ortho-iodoanilines 159 catalyzed by Pd@PS, reported in 2016 by Das and co-workers.

Polyanilines (PANIs) are conductive polymers with good thermal and chemical stability, which have found considerable attention as a support for transition metal catalysts. In 2010, Abdollahi-Alibeik et al. showed the application of ferric chloride-doped polyaniline nanoparticles $\left(\mathrm{FeCl}_{3} / \mathrm{PANI}\right)$ as an efficient recyclable Lewis acid catalyst for the synthesis of 2-substituted benzimidazoles by the reaction of ortho-phenylenediamines with aldehydes [122]. Under optimized conditions, reactions were carried out at room temperature in ethanol as a sustainable solvent for 30-240 min; moreover, $\mathrm{FeCl}_{3}$ /PANI showed the advantage of an easy recovery (consisting of a simple filtration, washing with $\mathrm{H}_{2} \mathrm{O}$ and acetone and final drying), which allowed for good recycling up to four runs with no loss of catalytic activity. Very recently, Bhalla and co-workers described the preparation of a bimetallic gold-copper oxide supported on polyaniline (named PANI@Au:CuO by the authors) as a recyclable photocatalyst for the construction of 2-arylbenzimidazole derivatives 163 under mild conditions by sequential amination/azidation/annulation reactions of toluene derivatives 161 with anilines 162 and azidotrimethylsilane (TMSN 3 ) (Scheme 49) [123]. The protocol, performed using 1.0 mol\% of PANI@Au:CuO under visible light irradiation, at room temperature in DMSO as the solvent for 10-18 h, was very appealing since it complies with most of the Twelve Principles of Green Chemistry, thanks to the use of 
recoverable catalysts (Principle 1: Prevention of Waste; Principle 4: Designing Safer Chemicals; Principle 8: Catalysis), visible light irradiation as a non-conventional energy source (Principle 6: Design for Energy Efficiency) and a sustainable reaction medium (Principle 5: Safer Solvents and Auxiliaries).

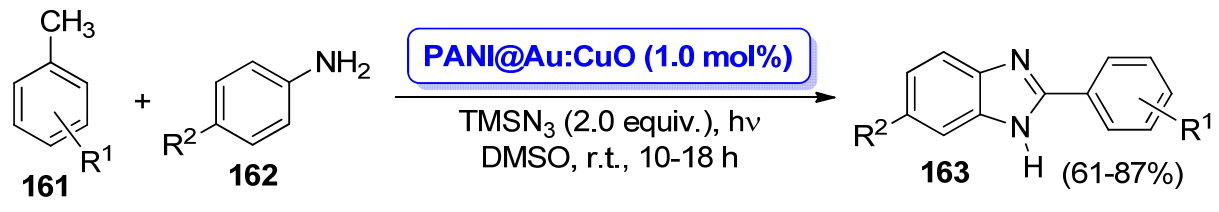

$\mathrm{R}^{1}=\mathrm{H}, 4-\mathrm{Me}, 4-\mathrm{OMe}, 4-\mathrm{Br}, 4-\mathrm{Cl}, 4-\mathrm{NO}_{2}$, 3-Me, 2-Me, 3,5-Me $\mathrm{M}_{2} \quad \mathrm{R}^{2}=\mathrm{H}, \mathrm{Br}, \mathrm{Cl}, \mathrm{NO}_{2}, \mathrm{Me}$

Scheme 49. Synthesis of 2-arylbenzimidazoles 163 by sequential amination/azidation/annulation reactions of toluene derivatives 161 with anilines 162 catalyzed by PANI@Au:CuO, reported in 2019 by Bhalla and co-workers.

Polymeric resins containing sulfur-based functional groups are widely used as a support for metal catalysts, thanks to the ability of sulfur atoms to stabilize metal nanoparticles. In this context, in 2011 Mandal, Dash and co-workers reported an easy method for the preparation of palladium nanoparticles embedded into a poly(1,4-phenylene sulfide) matrix (indicated below as PdNPs-PSS) by thermolysis of $\mathrm{Pd}(\mathrm{OAc})_{2}$ at $95^{\circ} \mathrm{C}$ for $4 \mathrm{~h}$ in toluene [124]. Interestingly, this material was successfully applied as a recoverable catalyst for the synthesis of 2,4-disubstituted pyrimidines 168 by a tandem acyl Sonogashira coupling of acid chlorides 164 and trimethylsilylacetylene 165, followed by the reaction of resulting propynone intermediates 166 with guanidinium salt 167 (Scheme 50). The protocol was carried out using 1.0 equiv. of acid chlorides 164, alkyne 165 and triethylamine, with $1.7 \mathrm{~mol} \%$ of PdNPs-PSS and in dry DMF as the solvent at room temperature; after completion of the first step, 2.5 equiv. of guanidinium salt 167, 3.5 equiv. of $\mathrm{NaHCO}_{3}$ as the base and methanol as a co-solvent were added and refluxed up to quantitative conversion. Although the synthetic approach was very interesting, PdNPs-PSS showed significant leaching (about $2 \%$ of the palladium content, as testified by ICP-AES) during its use, thus limiting its recyclability and implying the presence of palladium waste in the final product.

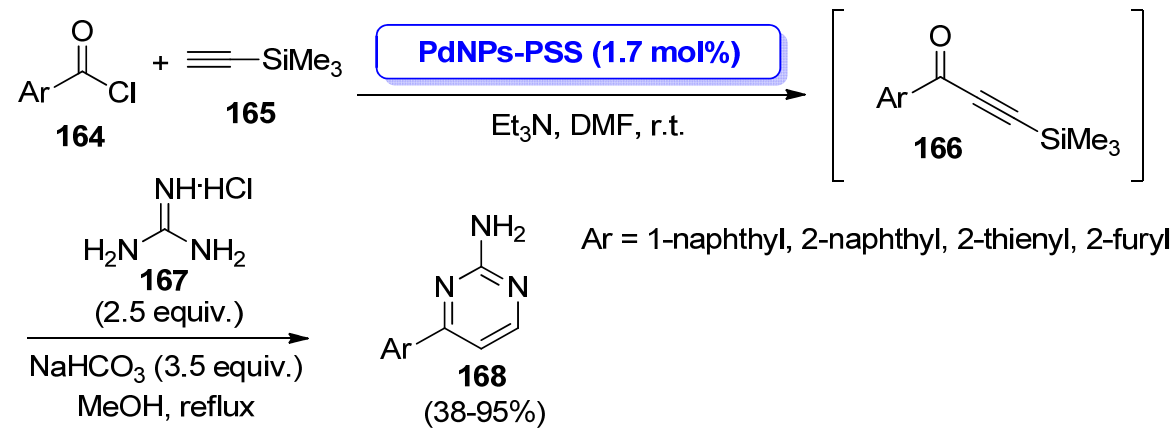

Scheme 50. Synthesis of 2,4-disubstituted pyrimidines 168 by tandem acyl Sonogashira/cyclization of acid chlorides 164, trimethylsilylacetylene 165 and guanidinium salt 167 catalyzed by PdNPs-PSS, reported in 2011 by Mandal, Dash and co-workers.

Commercially available mercapto-functionalized polyolefin fibers Smopex ${ }^{\circledR}-111$ and Smopex ${ }^{\circledR}-234$ are typical scavengers of precious metals, which have also been successfully used as supports for metal catalysts. Very recently, our research group described the preparation of palladium nanoparticles immobilized on Smopex ${ }^{\circledR}-111$ and Smopex ${ }^{\circledR}-234$ fibers (named, respectively, Pd/Smopex ${ }^{\circledR}-111$ and $\mathrm{Pd} /$ Smopex $^{\circledR}-234$ ) and their application in $\mathrm{Cu}$-free cyclocarbonylative Sonogashira reactions for the synthesis of heterocycles [125]. Pd nanoparticles were obtained by the Metal Vapor Synthesis (MVS) technique [126,127], generating metal nanoclusters weakly stabilized by organic solvents, which were then dis- 
persed on Smopex ${ }^{\circledR}$ supports by simple impregnation at $25^{\circ} \mathrm{C}$ (Scheme 51a). In particular, $\mathrm{Pd} /$ Smopex ${ }^{\circledR}-234$ was found to be very efficient for the synthesis of 1-alkylideneisoindoline 172 and dihydrobenzazepine 173 by the reaction of, respectively, 2-ethynylbenzyl tosylamide 169 and 2- ethynylhomobenzyl tosylamide 170 with iodobenzene 171, performed under a $\mathrm{CO}$ atmosphere (20 atm) in $\mathrm{Et}_{3} \mathrm{~N}$ with toluene as the reaction medium, at $100{ }^{\circ} \mathrm{C}$ for $24 \mathrm{~h}$ (Scheme 51b). Preliminary tests of metal leaching revealed that a very low palladium content $(0.8 \mathrm{w} / \mathrm{w} \%$ of the starting amount) was found in the reaction mixture after the removal of $\mathrm{Pd} / \mathrm{Smopex}{ }^{\circledR}-234$ catalyst by hot filtration, thus suggesting the possibility of good recyclability.

(a)
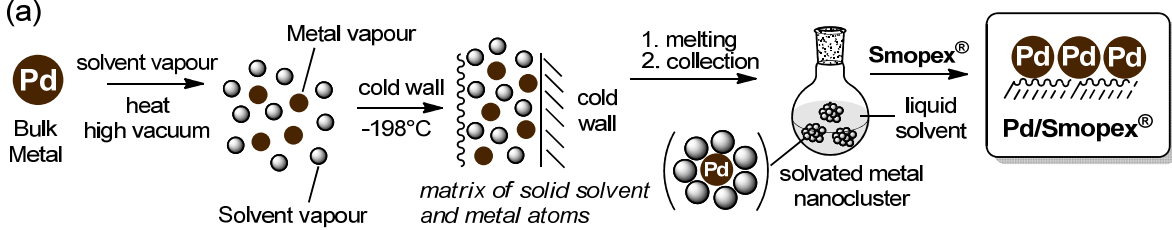

(b)

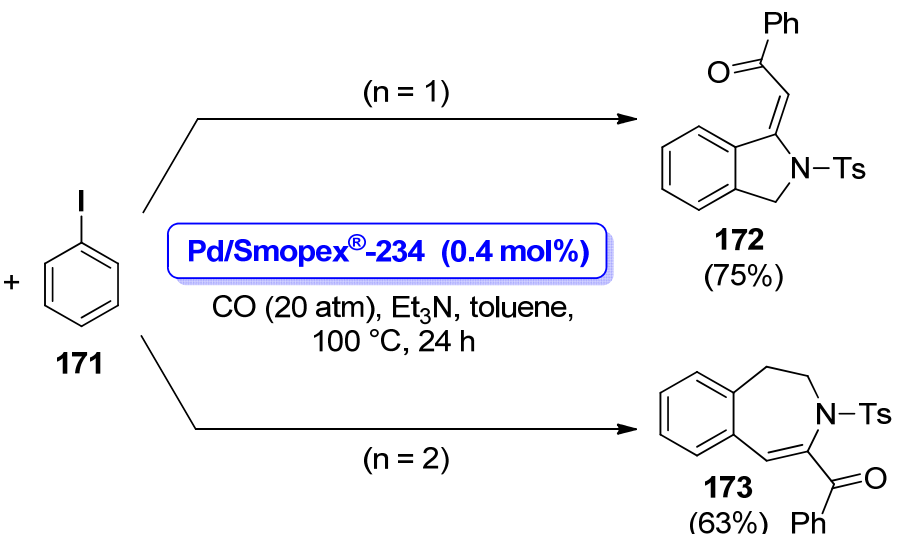

Scheme 51. (a) Schematic route for the preparation of Pd nanoparticles supported on Smopex ${ }^{\circledR}-234$ by Metal Vapor Synthesis. (b) Synthesis of 1-alkylideneisoindoline $\mathbf{1 7 2}$ and dihydrobenzazepine $\mathbf{1 7 3}$ by cyclocarbonylative Sonogashira of 2-ethynyl(homo)benzyl tosylamides 169-170 with iodobenzene 171 catalyzed by Pd/Smopex ${ }^{\circledR}-234$, reported in 2021 by Aronica et al.

Other synthetic organic polymers have only occasionally been adopted as a support for metal catalysts. In 2013, Rad, Behrouz et al. proposed the use of nanosized copper(I) oxide supported on a reticulated melamine-formaldehyde resin (named nano- $\mathrm{Cu}_{2} \mathrm{O}-\mathrm{MFR}$ ) for the 1,3-dipolar cycloaddition of $\beta$-azido alcohols with alkynes to give $1 H$-triazoles with antibacterial activity [128]. In 2019, Sasidharan and co-workers reported the preparation of a copper catalyst supported on a hierarchical mesoporous organic polymer (indicated as $\mathrm{Cu}-\mathrm{HMOP}$ by the authors) with nanorod morphology, showing high activity in the synthesis of quinolines by multi-step oxidative dehydrogenative coupling of ortho-aminobenzyl alcohol with aryl ketones $\left(t-\mathrm{BuOK}, \mathrm{O}_{2}\right.$, toluene, $\left.130{ }^{\circ} \mathrm{C}, 24 \mathrm{~h}\right)$, as well as in the synthesis of 1,2,3-1H-triazoles by a tandem azidation/[3 + 2] cycloaddition reaction of benzyl halides and terminal alkynes $\left(\mathrm{NaN}_{3}, \mathrm{Et}_{3} \mathrm{~N}, \mathrm{H}_{2} \mathrm{O}\right.$, room temperature, $\left.18 \mathrm{~h}\right)$ [129]. Interestingly, hot filtration tests and ICP analysis of the filtrate revealed a truly heterogeneous mechanism for the $\mathrm{Cu}$-HMOP catalyst; moreover, its easy recoverability by centrifugation allowed an efficient recycling. In 2020, Islam et al. described a $\mathrm{CO}_{2}$ fixation catalyzed by the copper nanoparticles-incorporated covalent organic framework $(\mathrm{Cu}-\mathrm{NPs} @ \mathrm{COF})$ for the synthesis of benzimidazoles from $o$-phenylenediamines under moderate conditions $\left(\mathrm{CO}_{2} 1 \mathrm{~atm}\right.$,

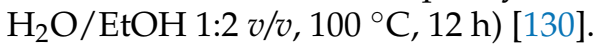

\subsection{Metal Catalysts Supported on Organic Polymer-Anchored Ligands}

An appealing approach for the development of supported metal catalysts is the immobilization of homogeneous complexes on organic matrices, carried out by covalent 
grafting of suitable ligands to synthetic organic polymers, followed by complexation of the desired metal species. In the context of the synthesis of $N$-heterocycles, to the best of our knowledge the first study was reported in 2000 by Grigg et al.: they reported the use of a palladium species coordinated to diphenylphosphinated DVB-crosslinked polystyrene (simply named PS-Pd by the authors), in combination with a homogeneous ruthenium benzylidene complex, in a cascade ring-closing metathesis/intramolecular Heck reaction protocol for the synthesis of bridged bicyclic and tricyclic nitrogen-containing heterocycles [131]. It is worth emphasizing that the use of a supported palladium catalyst was due in order to optimize the cascade protocol (since it did not work using both rhodium and palladium homogeneous catalysts), rather than the need of recoverable and recyclable catalytic systems.

However, nitrogen-based ligands are definitely the most used for this class of catalytic systems. Concerning anchored amine ligands, Bakherad and co-workers reported in 2009 the use of a polystyrene-supported palladium(II) ethylenediamine complex (simply named PS-en-Pd(II)) for the synthesis of 2-benzylimidazo[2,1-b]pyridines $\mathbf{1 7 6}$ through a tandem Sonogashira coupling/heteroannulation of 2-amino-1-(2-propynyl)pyridinium bromide 174 with aryl iodides 175 [132]. Reactions were carried out under typical Sonogashira coupling conditions, using $3 \mathrm{~mol} \%$ of PS-en-Pd(II) as the catalyst, $20 \mathrm{~mol} \%$ of $\mathrm{CuI}$ as the cocatalyst, $3 \mathrm{~mol} \%$ of $\mathrm{PPh}_{3}$ as an additional ligand and 3.0 equiv. of $\mathrm{Et}_{3} \mathrm{~N}$ as the base, in DMF at room temperature for 12-16 h (Scheme 52). Although the recyclability of the PS-en-Pd(II) catalyst in five consecutive runs was tested, the protocol appeared to be unappealing from the Green Chemistry point of view, due to the use of homogeneous copper iodide as the co-catalyst, with the risk of metal contamination of the imidazo[2,1- $b]$ pyridine products, as well as the use of toxic DMF as the reaction medium.

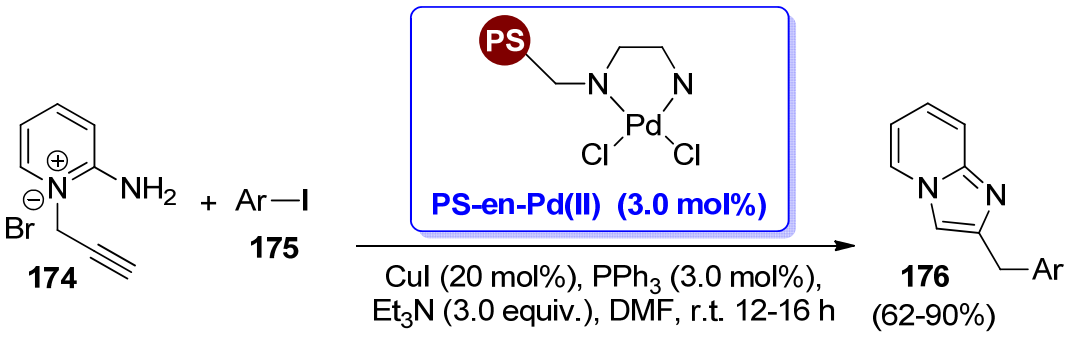

$\begin{aligned} \mathrm{Ar}= & 2-\mathrm{NO}_{2}-\mathrm{C}_{6} \mathrm{H}_{4}, 3-\mathrm{NO}_{2}-\mathrm{C}_{6} \mathrm{H}_{4}, 4-\mathrm{NO}_{2}-\mathrm{C}_{6} \mathrm{H}_{4}, 2-\mathrm{Me}-4-\mathrm{NO}_{2}-\mathrm{C}_{6} \mathrm{H}_{3}, 2-\mathrm{NO}_{2}-4-\mathrm{Cl}_{2} \mathrm{C}_{6} \mathrm{H}_{3}, \\ & 3-\mathrm{NO}_{2}-4-\mathrm{Cl}-\mathrm{C}_{6} \mathrm{H}_{3}, 4-\mathrm{CN}-\mathrm{C}_{6} \mathrm{H}_{4}, 4-\mathrm{COMe}-\mathrm{C}_{6} \mathrm{H}_{4}, 4-\mathrm{COOMe}-\mathrm{C}_{6} \mathrm{H}_{4}\end{aligned}$

Scheme 52. Synthesis of imidazo[2,1-b]pyridines 176 via tandem Sonogashira coupling/heteroannulation of 2-amino-1-(2-propynyl)pyridinium 174 with aryl iodides $\mathbf{1 7 5}$ catalyzed by PS-en-Pd(II), reported in 2009 by Bakherad and co-workers.

More recently, Islam et al. reported the development of a polystyrene-anchored bidentate amino acid ligand, used for the complexation of a ruthenium(III) species by treatment with $\mathrm{RuCl}_{3}$ in refluxing DMF for $24 \mathrm{~h}$ (Scheme 53a) [133]. The resulting Rusupported material (indicated below PS-L-Ru) was used as a catalyst for the preparation of 1,4-disubstituted 1,2,3-1H-triazoles 179 by sequential azide formation/[3 + 2] cycloaddition of benzyl bromides 177 and terminal alkynes 178, carried out at room temperature and in water as the solvent (Scheme 53b). Interestingly, several studies were carried out in order to evaluate the reaction mechanism (homogeneous vs. heterogeneous) of the PS-L-Ru catalyst: recycling tests, performed by simply catalyst filtration, washing and drying, revealed the same catalytic activity for six runs; hot filtration tests of the reaction mixture at incomplete conversion and ICP-AAS analysis of the filtrate revealed the absence of any ruthenium leaching. These results suggested a merely heterogeneous mechanism, together with high thermal and chemical stability under the reaction conditions: features making this system compliant with many of the Twelve Principles of Green Chemistry. 
(a)

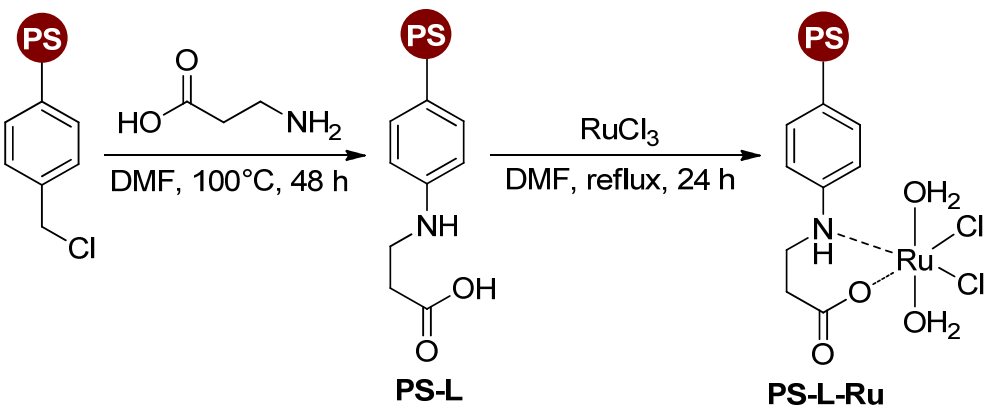

(b)

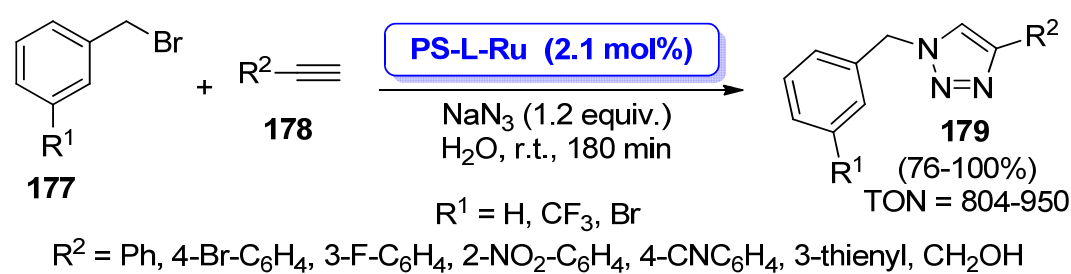

Scheme 53. (a) Schematic route for the preparation of PS-L-Ru catalyst. (b) Synthesis of 1,2,3-triazoles $\mathbf{1 7 9}$ by sequential azide formation/[3 + 2] cycloaddition of benzyl bromides $\mathbf{1 7 7}$ and terminal alkynes 178 catalyzed by PS-L-Ru, reported in 2015 by Islam et al.

Nitrogen atoms of $N$-heterocyclic compounds are typical ligands for metal complexes; therefore, it is not surprising that $N$-heterocycles have been successfully immobilized to organic polymers in order to obtain efficient and easily recoverable supports for transition metal catalysts. In 2009, Taran, Quignard et al. reported the synthesis of 1,2,3-1H-triazoles by [3 + 2] cycloaddition of organic azides and terminal alkynes catalyzed by a Cu(I) species complexed on a chitosan-functionalized phenanthroline ligand; the reactions worked well both in water and ethanol as the reaction medium, at $70{ }^{\circ} \mathrm{C}$ for $12 \mathrm{~h}$ with $0.1 \mathrm{~mol} \%$ of $\mathrm{Cu}$ loading; few tests were also carried out under microwave irradiation, in $\mathrm{EtOH} / \mathrm{Et}_{3} \mathrm{~N}$ 95:5 $v / v$ for $15 \mathrm{~min}$ [134].

Tris-(benzyltriazolylmethyl)amine (TBTA) is a N-heterocyclic ligand for copper(I) species, typically used in the [3 + 2] azide-alkyne cycloaddition under homogeneous conditions. However, the TBTA ligand has been successfully immobilized on synthetic organic polymers in order to obtain supported copper catalysts for the synthesis of 1,2,3-1H-triazoles. In 2007, Fokin and co-workers reported the immobilization of the TBTA ligand on a TentaGel ${ }^{\circledR}$ resin, followed by metal loading with $\left[\mathrm{Cu}(\mathrm{MeCN})_{4}\right] \mathrm{PF}_{6}$ to give the final supported catalytic system TG-TBTA-CuPF 6 [135]. Such catalysts were then tested $(1.0 \mathrm{~mol} \%$ of copper loading) for the [ $3+2]$ cycloaddition reaction of azides $\mathbf{1 8 0}$ with terminal alkynes 181 (1.1 equiv.), performed in methanol at room temperature for $16-24 \mathrm{~h}$ to give triazoles 182 in excellent yields, mostly in yields $>90 \%$ (Scheme 54). The authors examined the recyclability of TG-TBTA-CuPF 6 resin in the reaction of benzyl azide with phenylacetylene. Interestingly, catalytic activity was unchanged for six consecutive cycles, while the yield of the triazole product remained $>90 \%$ up to the 10 th run. From this study, it was clear that the TG-TBTA support showed strong affinity for copper, thus preventing the metal contamination of $1 \mathrm{H}$-triazole products.

Very recently, Mandoli et al. reported the covalent immobilization of TBTA on highly cross-linked polystyrene resins, obtained in the form of conventional beads (PS-TBTA-B) and porous monoliths (PS-TBTA-M) by, respectively, suspension and mold copolymerization of a TBTA-like monomer with styrene and divinylbenzene (Scheme 55a) [136]. Both materials were then charged with different $\mathrm{Cu}(\mathrm{I})$ or $\mathrm{Cu}$ (II) salts (in the last case together with a proper reducing agent), and tested in the [3 + 2] cycloaddition reaction of benzyl azides 183 with alkynes 184 to give $1 H$-triazoles 185. In particular, copper species supported on beads resin (PS-TBTA-B $\cdot \mathrm{CuX}_{1-2}$ ) were used in batch conditions, while copper species supported on porous monoliths resin (PS-TBTA-M $\cdot \mathrm{CuX}_{1-2}$ ) were used under continuous flow conditions (Scheme 55b). 


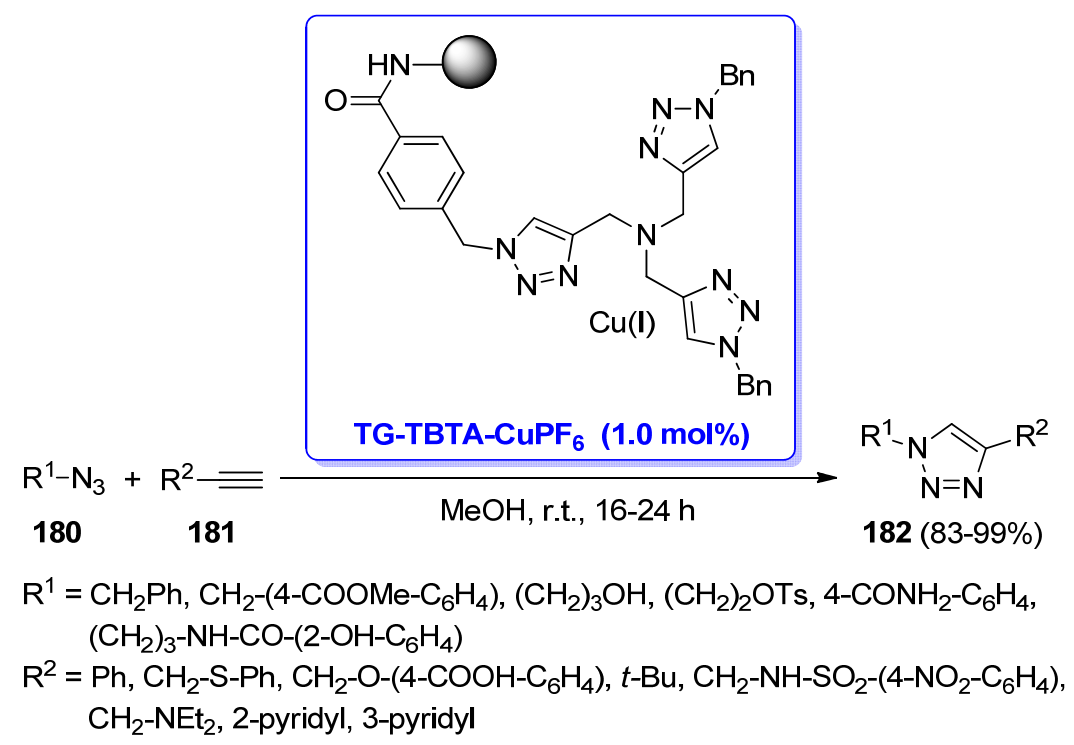

Scheme 54. Synthesis of 1,2,3-triazoles 182 by [3 + 2] azide-alkyne cycloaddition of azides 180 with terminal alkynes 181 catalyzed by TG-TBTA-CuPF 6 , reported in 2007 by Fokin and co-workers.
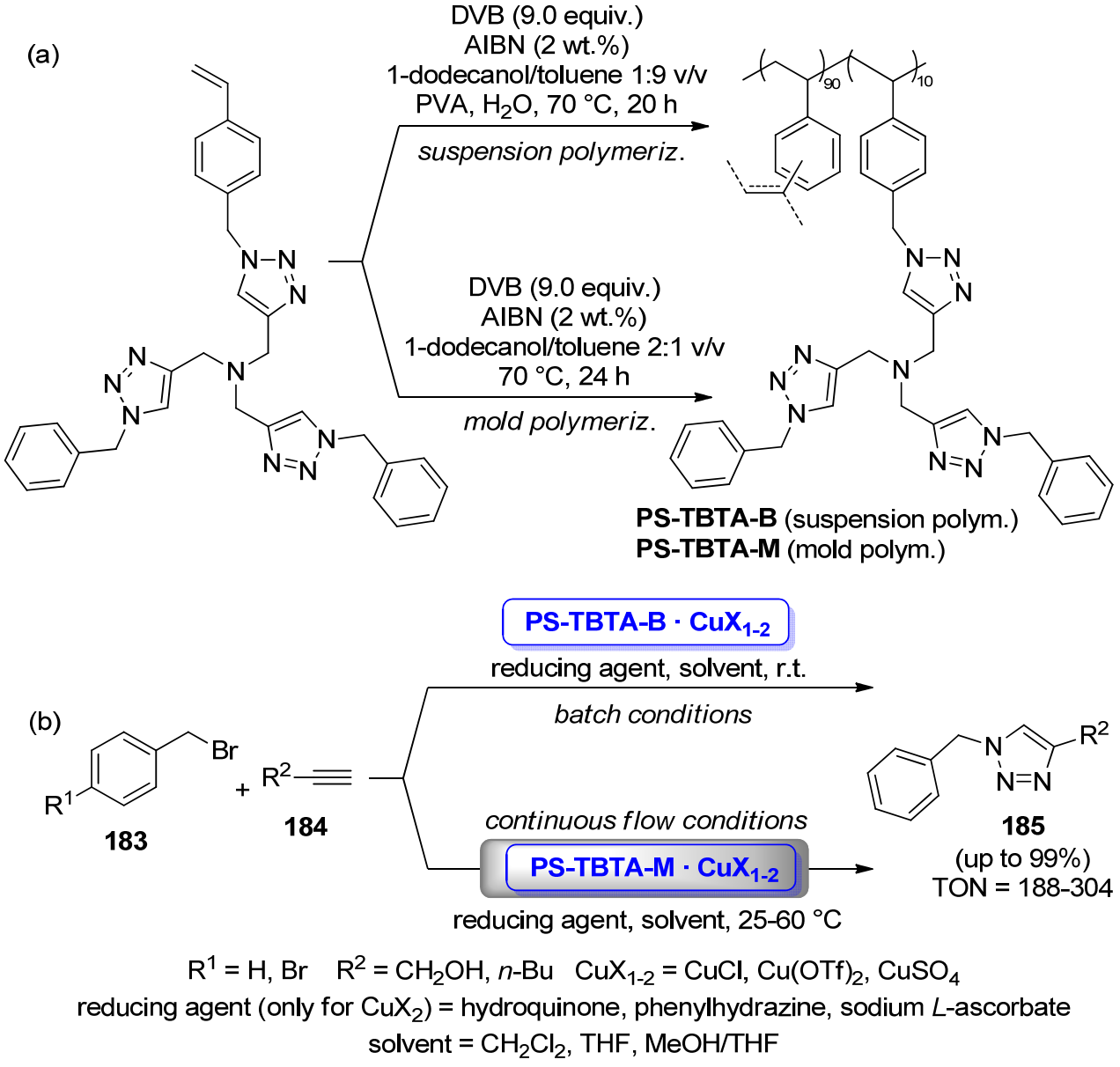

Scheme 55. (a) Schematic route for the immobilization of TBTA on cross-linked polystyrene resins, in the form of beads (PS-TBTA-B) and porous monoliths (PS-TBTA-M). (b) Synthesis of triazoles 185 by [3 + 2] cycloaddition of azides 183 with alkynes 184 catalyzed by PS-TBTA-B $\cdot$ CuX 1-2 $_{\text {(batch }}$ conditions) or PS-TBTA-M $\cdot \mathrm{CuX}_{1-2}$ (continuous flow conditions), reported in 2020 by Mandoli et al. 
Other classes of nitrogen-based ligands anchored to organic polymers have been less investigated as supports of metal catalysts for the preparation of $N$-heterocycles. In 2007, in the context of an extended study on Mizoroki-Heck reactions of allyl alcohols, Nájera et al. reported several examples of the one-pot synthesis of quinolines via Mizoroki-Heck vinylation or indoles via sequential $N$-allylation/Mizoroki-Heck vinylation of 2-iodoaniline with allyl alcohols catalyzed by a palladium species complexed on Kaiser oxime resin (1 mol\%), performed in $\mathrm{H}_{2} \mathrm{O}$ or DMA/ $\mathrm{H}_{2} \mathrm{O}$ as a reaction medium and in the presence of $\mathrm{Cy}{ }_{2} \mathrm{NMe}$ as a base (1.5 equiv.) and TBAB as an additive (1.0 equiv.) [137]. In 2019, Islam and co-workers reported the preparation of a zinc(II) catalyst supported on a polystyrene-anchored salen ligand (named PS-Zn(II)SALTETA by the authors) and its application in the synthesis of benzimidazoles 187 by $\mathrm{CO}_{2}$ insertion of ortho-phenylenediamines 186 [138]. The protocol was performed with $3.0 \mathrm{~mol} \%$ of the PS-Zn(II)SALTETA catalyst, dimethylamine borane (DMAB, 3.0 equiv.) as the base, under a $\mathrm{CO}_{2}$ atmosphere $(1 \mathrm{~atm})$, in a $\mathrm{H}_{2} \mathrm{O} / \mathrm{EtOH} \mathrm{1:2} \mathrm{(v/v)}$ mixture as the solvent, at $100{ }^{\circ} \mathrm{C}$ for $24 \mathrm{~h}$ (Scheme 56). The protocol was very appealing from the point of view of Green Chemistry, thanks to the use of recoverable catalysts (Principle 1: Prevention of Waste; Principle 4: Designing Safer Chemicals; Principle 8: Catalysis) and sustainable solvents (Principle 5: Safer Solvents and Auxiliaries), as well as the fixation of $\mathrm{CO}_{2}$, which represents the vast majority of greenhouse gas emissions (Principle 1: Prevention of Waste; Principle 2: Atom Economy).

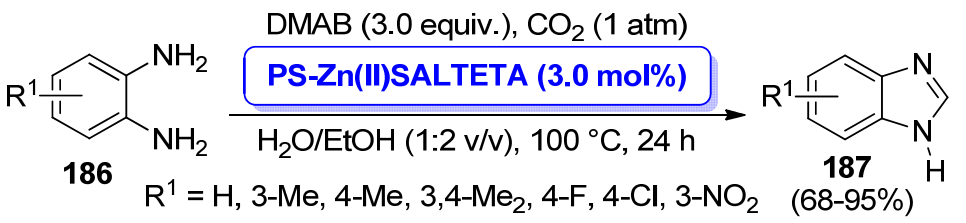

Scheme 56. Synthesis of benzimidazoles 187 by $\mathrm{CO}_{2}$ insertion of ortho-phenylenediamines 186 catalyzed by PS-Zn(II)SALTETA, reported in 2019 by Islam and co-workers.

Moving on to oxygen- and carbon-based ligands anchored to organic polymers, only very few examples of supported metal catalysts have been reported in the context of the synthesis of $N$-heterocycles. In fact, most of them are based on catalytic systems anchored to soluble polymers, which are outside the scope of this review. In 2003, Buchmeiser et al. reported the immobilization of a chiral Schrock's molybdenum catalyst into a insoluble polymer backbone via ring-opening metathesis polymerization of a norborn-2-ene-substituted chiral phenoxide ligand, followed by the complexation of the suitable Mo precursor (Scheme 57a) [139]. Such a catalyst was successfully used for enantioselective ring-closing olefin metathesis of prochiral or racemic substrates for the synthesis of chiral heterocycles, including tetrahydropyridines 189 from racemic amines 188. Reactions were carried out in $\mathrm{CH}_{2} \mathrm{Cl}_{2}$ for $30 \mathrm{~min}$ at $20{ }^{\circ} \mathrm{C}$, using $2.8-5.2 \mathrm{~mol} \%$ of the supported Schrock's molybdenum catalyst, giving heterocyclic products 189 in modest yields but good enantiomeric excess (Scheme 57b). The authors also investigated the recyclability of the catalytic system, made possible by simple filtration, washing and drying; after three consecutive runs, ICP-OES analysis revealed only a small decrease $(<5 \%)$ in the Mo content in the polymeric matrix.

In 2016, the synthesis of several dihydropyrroles and tetrahydropyridines by ring closing metathesis was described by Bazzi and co-workers with the use of a polyisobutylene- supported bis(arylimino)acenaphthene $N$-heterocyclic carbene Ru complex (PIB-BIAN-NHC-Ru) as the recoverable catalyst [140]. The protocol was developed in $n$-heptane as the solvent, at room temperature and in the presence of $1 \mathrm{~mol} \%$ of the PIB-BIAN-NHC-Ru catalyst, affording the desired $N$-heterocyclic products in excellent yields after only $1 \mathrm{~h}$. One of the most appealing features of this study is the high recyclability of the supported ruthenium species; in fact, it was used in eight consecutive runs with no significant loss of catalytic activity. Despite the high recyclability of PIB-BIAN-NHC-Ru, non-negligible metal leaching was observed in the reaction mixture (after catalyst removal by filtration) at the end of each cycle, thus limiting the impact of the present protocol in the context of Green Chemistry. 
(a)<smiles>C=CC1CC(CO)C(COCc2cc(C(C)(C)C)c(O)c(-c3c(C)c(COCC4C(C=C)CC(C=C)C4COCc4cc(CBr)c(O)c(-c5c(O)c(CBr)cc(C(C)(C)C)c5O)c4O)cc(C(C)(C)C)c3O)c2C)C1</smiles><smiles>Cc1c(C(C)(C)C)cc(COCC2CC3C=CC2C3)c(O)c1-c1c(C)c(COCC2CC3C=CC2C3)cc(C(C)(C)C)c1O</smiles><smiles>CCOC(=O)N1CCN(C)C1CN(C)S(C)(=O)=O</smiles><smiles>C=COCC</smiles>

$\mathrm{CH}_{2} \mathrm{Cl}_{2}, 45^{\circ} \mathrm{C}, 18 \mathrm{~h}$

(b)<smiles>[R]C(=C)CC(c1ccccc1)N(CC([R])=C)c1ccccc1</smiles>

\section{Schrock catalyst (2.8-5.2 mol\%)}

$$
\begin{gathered}
\mathrm{CH}_{2} \mathrm{Cl}_{2}, 20{ }^{\circ} \mathrm{C}, 30 \min \\
\mathrm{R}^{1}=\mathrm{H}, \mathrm{Me} \quad \mathrm{R}^{2}=\mathrm{H}, \mathrm{Me}
\end{gathered}
$$

Chiral Schrock catalyst

Scheme 57. (a) Schematic route for the immobilization of chiral Schrock's Mo catalyst into a insoluble polymer backbone. (b) Synthesis of optically active tetrahydropyridines $\mathbf{1 8 9}$ by ring closing metathesis of racemic $\mathbf{1 8 8}$ catalyzed by the supported chiral Schrock's Mo catalyst, reported in 2003 by Buchmeiser et al.

\subsection{Metal Catalysts Supported on Biopolymers}

The interest in natural biopolymers (in particular, chitosan, cellulose, alginate, lignin, wool and silk) as insoluble supports for metal catalysts [141-144] is more recent with respect to the other abovementioned classes of organic matrices, as it is closely related to the increasing impact of environmentally friendly experimental conditions in the context of organic synthesis. These materials are typically non-toxic, cheap and environmentally friendly, but they also offer several opportunities for functionalization, which allow for a fine tuning of their physico-chemical properties. However, studies on the preparation of $N$-heterocycles based on the use of metal catalysts supported on natural biopolymers are still quite limited.

The first study was reported in 2013 by Khalafi-Nezhad et al., wherein chitosansupported magnetic ionic liquid nanoparticles (CSMIL) were developed as a heterogeneous iron catalyst ( $2.5 \mathrm{~mol} \%$ of loading) for the solvent-free synthesis of 5 -substituted $1 \mathrm{H}$-tetrazoles by the reaction of $\mathrm{NaN}_{3}$ with benzonitriles, performed for $6 \mathrm{~h}$ at $70{ }^{\circ} \mathrm{C}$, and 1-substituted $1 \mathrm{H}$-tetrazoles by the reaction of $\mathrm{NaN}_{3}$ with anilines and triethyl ortho-formate, carried out for $1 \mathrm{~h}$ at $70{ }^{\circ} \mathrm{C}$ [145]. The possibility of working without any solvent, in combination with the good recyclability of the CSMIL catalyst (successfully tested in the reaction of 4- nitrobenzonitrile with $\mathrm{NaN}_{3}$ up to 5 runs with no significant loss of catalytic activity), makes the present protocol quite interesting from the point of view of Green Chemistry. In the same year, Varma and co-workers described an easy method for the immobilization of copper sulfate on a chitosan biopolymer (which was named Chit- $\mathrm{CuSO}_{4}$ by the authors), by simply stirring a suspension of chitosan in water with $\mathrm{CuSO}_{4}$ for $3 \mathrm{~h}$ at room temperature [146]. Interestingly, Chit-CuSO 4 was found to be very effective for the synthesis of 1,2,3-1H-triazoles 192 by [3 + 2] cycloaddition of benzyl azides 190 and phenylacetylenes 191, carried out in water as the solvent, at room temperature for 4-6 h, with a metal loading of only $0.4 \mathrm{~mol}_{\%}$ (Scheme 58 ). The Chit-CuSO 4 catalyst was removed from the reaction mixture by simple filtration and reused in five consecutive runs 
with no decrease in its catalytic activity; interestingly, ICP-AES analysis of the catalyst, freshly prepared and after the fifth cycle, revealed almost the same copper concentration (5.1 wt.\% vs. 5.02 wt.\%), thus confirming the absence of metal leaching into the solution.

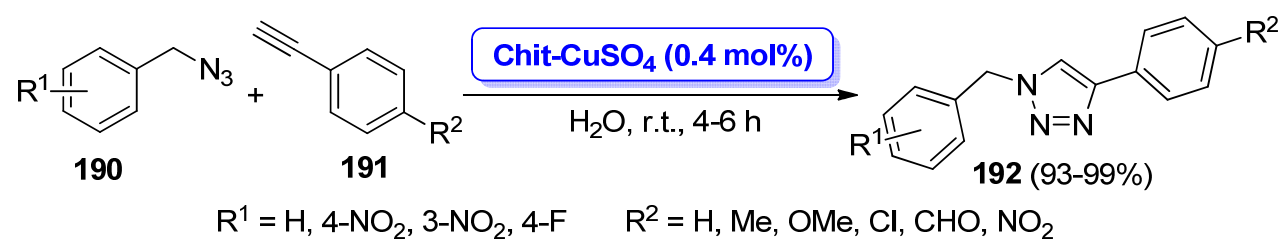

Scheme 58. Synthesis of $1 H$-triazoles 192 by [3 + 2] cycloaddition of benzyl azides 190 and phenylacetylenes 191 catalyzed by Chit-CuSO 4 , reported in 2013 by Varma and co-workers.

In 2020, Gu, Bai and co-workers reported the immobilization of a $\mathrm{Cu}$ species on modified lignosulfonate and its application as a recoverable catalyst for the preparation of nitrogen-containing heterocycles [147]. Sodium lignosulfonate, a typical waste biomass from the paper-making industry containing aryl and sodium sulfonate groups, was treated with sodium 2-formylbenzenesulfonate, followed by ion exchange and acidification; the phenylaldehyde condensation product was then used as a robust support (LS-FAS) for the immobilization of copper by treatment with $\mathrm{Cu}(\mathrm{OTf})_{2}$ in refluxing ethanol. The final material (called LS-FAS-Cu) was successfully applied in the preparation of several classes of compounds: tricyclic $1 H$-indole derivatives by one-pot multicomponent reactions, 2-aryl pyridines by oxidative cyclization of diamines with ketones, and 3-aryl isoquinolines by cyclocondensation of ortho-alkynyl aromatic aldehydes with urea. Despite the easy recoverability by filtration, recyclability tests of LS-FAS-Cu revealed a decrease in catalytic activity starting from the third run; moreover, reaction conditions often required high temperatures (i.e., $>100{ }^{\circ} \mathrm{C}$ ), thus limiting their advantages in the context of Green Chemistry.

\section{Metal Catalysts Supported on Hybrid Inorganic-Organic Matrices for the Synthesis of $N$-Heterocycles}

In the last part of this review, we will take into account all the synthetic protocols for the preparation of $N$-heterocycles involving the use of metal catalysts supported on hybrid inorganic-organic matrices, whose introduction in heterogeneous catalysis is definitely more recent with respect to conventional inorganic or organic supports. However, it is worth emphasizing that several supports with different physico-chemical features belong to this class: organic ligands anchored to inorganic matrices (i.e., the same as those mentioned in the first part of the manuscript: carbon, silica, metal oxides, minerals, magnetic core/inorganic shell composite), which offered novel opportunities for heterogenization of homogeneous complexes via covalent functionalization or non-covalent interactions; magnetic core/organic shell composites, acting similarly to cross-linked polymeric matrices but with the advantage of easy separation from the reaction mixture using an external magnet; metal organic frameworks, an emerging class of porous materials that represent the last frontier of hybrid inorganic-organic supports, showing excellent thermal and chemical stability and highly tunable structures. In this third section of the paper, we will organize the literature depending on the type of support following the above-mentioned order.

\subsection{Metal Catalysts Supported on Carbon-Anchored Ligands}

As already emphasized in Section 2.1, carbon-derived materials such as carbon nanotubes and reduced graphene oxide have been widely used as supports for the preparation of heterogeneous catalysts. In 2012, Sharghi, Doroodmand et al. reported the synthesis of a terpyridine $\mathrm{Cu}(\mathrm{II})$ complex (Cu(II)-PhTPY) and its covalent immobilization to activated multiwalled carbon nanotubes, to produce the supported catalyst AMWCNTs-O-Cu(II)-PhTPY as a black solid, which was then applied to the synthesis of $\beta$-hydroxy-1,4-disubstituted triazoles 195 by the tandem azide formation/[ $3+2]$ cycloaddition reaction of epoxides 193 with terminal alkynes 194 [148]. Reactions were carried out with equimolar amounts of 
193 and 194 and a slight excess of sodium azide, in the presence of $2.0 \mathrm{~mol} \%$ of supported catalyst loading, at room temperature for $1-6 \mathrm{~h}$ using $\mathrm{H}_{2} \mathrm{O}$ as the solvent, affording the expected triazoles in good to excellent yields (Scheme 59). Under similar experimental conditions, 1,4-disubstituted 1,2,3-1H-triazoles were also generated using benzyl or alkyl halides instead of epoxides. The AMWCNTs-O-Cu(II)-PhTPY catalyst was also very appealing from the Green Chemistry point of view, as the authors hypothesized a merely heterogeneous mechanism, supported by recyclability tests (five consecutive runs with the same catalytic activity) and ICP analysis (Cu content of $4.895 \mathrm{wt}$. \% for freshly prepared samples and $4.832 \mathrm{wt}$.\% for the same catalyst after the fifth run).

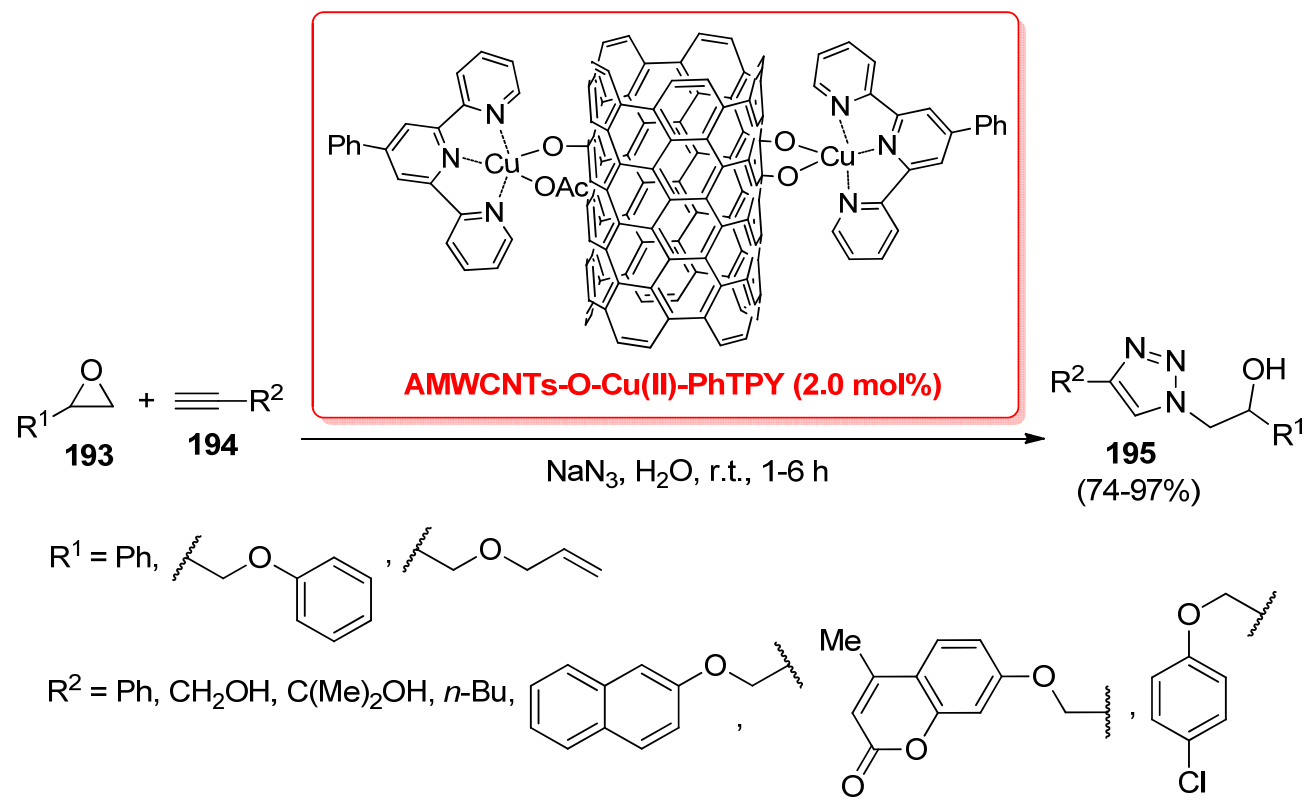

Scheme 59. Synthesis of $\beta$-hydroxy-1,4-disubstituted triazoles 195 by tandem azide formation/[3 + 2] cycloaddition reaction of epoxides 193 with alkynes 194 catalyzed by AMWCNTs-O-Cu(II)-PhTPY, reported in 2012 by Sharghi, Doroodmand et al.

In a following study, the same research group explored the use of the same supported catalytic system AMWCNTs-O-Cu(II)-PhTPY for the synthesis of several $1 H$-tetrazole derivatives [149]. In particular, 5-substituted $1 H$-tetrazoles 197 were obtained by the reaction of benzonitriles 196 with $\mathrm{NaN}_{3}$ (1.3 equiv.), carried out with $4 \mathrm{~mol} \%$ of the catalyst in the presence of 1.0 equiv. of $\mathrm{NH}_{4} \mathrm{OAc}$, at $70{ }^{\circ} \mathrm{C}$ in $\mathrm{DMF}$ as the solvent; 1-substituted $1 \mathrm{H}$-tetrazoles 199 were instead synthesized by the reaction of anilines 198 with $\mathrm{NaN}_{3}$ (1.3 equiv.) and triethyl ortho-formate (1.0 equiv.), using $4 \mathrm{~mol} \%$ of the catalyst, at $70{ }^{\circ} \mathrm{C}$ in $\mathrm{H}_{2} \mathrm{O}$ (Scheme 60).

In 2009, Wang and co-workers employed single-walled carbon nanotubes (SWNTs) for the immobilization of a pyrene-tagged ruthenium carbene complex; very interestingly, they developed one of the first examples of a metal complex anchored to an inorganic matrix by non-covalent interactions, i.e., the $\pi-\pi$ stacking between the pyrene moiety of the complex and the surface of carbon nanotubes [150]. An extended study of ruthenium desorption from the carbon support of such a catalytic system (named SWNTs-Pyr-Ru) was performed in different solvents; if significant desorption of the ruthenium carbene complex was found in non-polar solvents (benzene, toluene, $\mathrm{CH}_{2} \mathrm{Cl}_{2}, \mathrm{THF}$ ), very minimal amounts leached into polar solvents such as ethyl acetate or acetone; moreover, no detectable desorption was observed in acetone at room temperature, while $\sim 80 \%$ leached after $24 \mathrm{~h}$ at $50{ }^{\circ} \mathrm{C}$. Starting from these results, SWNTs-Pyr-Ru was tested in representative ring-closing metathesis of functionalized dienes, including amides $\mathbf{2 0 0}$ and 201 for the synthesis of, respectively, dihydropyrroles 202 and tetrahydropyridines 203, performed for 5-12 h at 0-35 ${ }^{\circ} \mathrm{C}$ in acetone as the solvent (Scheme 61). Working under these experimental conditions, SWNTs- 
Pyr-Ru was efficiently recovered by filtration and reused without any further treatment up to seven consecutive runs. More recently, Schulz, Crévisy et al. proposed a similar noncovalent approach for the immobilization of several pyrene-tagged ruthenium complexes onto reduced graphene oxide, used as supported and recoverable catalysts (named rGO-Pyr$\mathrm{Ru}$ ) promoting the ring-closing metathesis of $N, N$-diallyl-4-methyl-benzenesulfonamide to give the corresponding dihydropyrrole product [151].

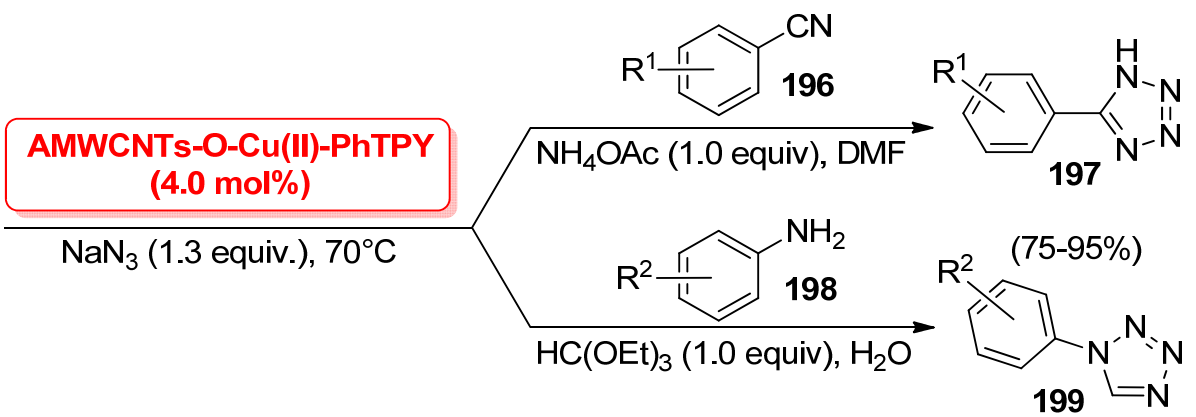

$\mathrm{R}^{1}=4-\mathrm{Me}$, 2-Me, 4-OMe, 4-NO $\mathrm{NO}_{2}$ 4- $\mathrm{CF}_{3}$, 4-Cl, 3-Cl, 4-Br, 3-Br, 4-CN, 3-CN, 2-CN

$\mathrm{R}^{2}=\mathrm{H}, 2-\mathrm{Me}$, 3-OMe, 4-OH, 4-Me, 4-i- $\mathrm{Pr}, 4-\mathrm{Cl}, 2-\mathrm{Cl}, 4-\mathrm{Br}, 3-\mathrm{Br}, 3-\mathrm{NO}_{2}$

Scheme 60. Synthesis of $1 \mathrm{H}$-tetrazoles 197 and 199 by reaction of $\mathrm{NaN}_{3}$ with, respectively, benzonitriles 196 and anilines 198 catalyzed by AMWCNTs-O-Cu(II)-PhTPY, reported in 2013 by Sharghi et al.

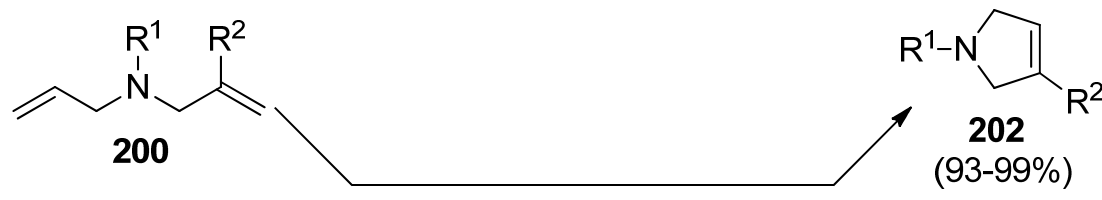

SWNTs-Pyr-Ru (0.2 mol\%)

acetone, $0-35{ }^{\circ} \mathrm{C}, 5-12 \mathrm{~h}$

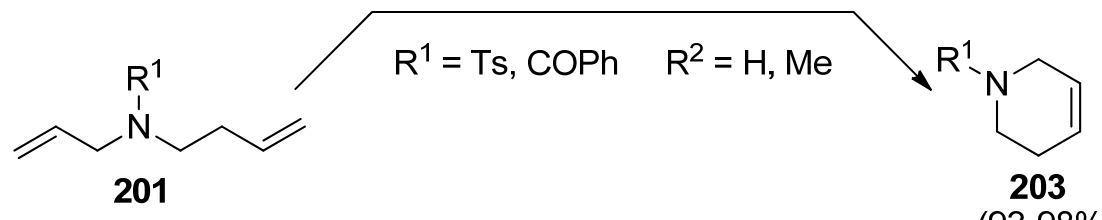

(93-98\%)

Scheme 61. Synthesis of dihydropyrroles 202 and tetrahydropyridines 203 by ring-closing metathesis of, respectively, amides 200 and 201 catalyzed by SWNTs-Pyr-Ru, reported in 2009 by Wang et al.

In 2015, Binder and co-workers described the preparation of several carbon-supported copper nanomaterials and their application as heterogeneous catalysts for the [3 + 2] cycloaddition reactions of benzyl azides with terminal alkynes [152]. The surface of graphene or carbon nanotubes was first functionalized with -OH groups by literature procedures; then, treatment with 1-chloro-2-isocyanatoethane allowed the chlorination of the carbon surface, followed by the reaction with $\mathrm{NaN}_{3}$. The resulting azide-functionalized carbon surfaces were then treated with 1-propargyl-3-methylimidazolium bromide by the click reaction, thus allowing for the attachment of imidazolium moieties; finally, the addition of copper(I) salt gave the final graphene-anchored and carbon nanotubes-anchored $\mathrm{N}$-heterocyclic carbene $\mathrm{Cu}(\mathrm{I})$ materials, called CRGO-Ima-Cu(I) and CNT-Ima-Cu(I), respectively (Scheme 62). In the [3 + 2] cycloaddition reactions of benzyl azides with terminal alkynes, full conversions were found only by using CRGO-Ima-Cu(I) at $2 \mathrm{~mol} \%$, while CNT-Ima-Cu(I) showed lower conversions even at $5 \mathrm{~mol} \%$; this result was tentatively attributed by the authors to the higher surface area of graphene, as well as to the better dispersion of copper complexes to the carbon surface. Interestingly, the CRGO-Ima-Cu(I) 
catalyst showed also high recyclability, with up to 10 consecutive runs with no significant loss of catalytic activity.

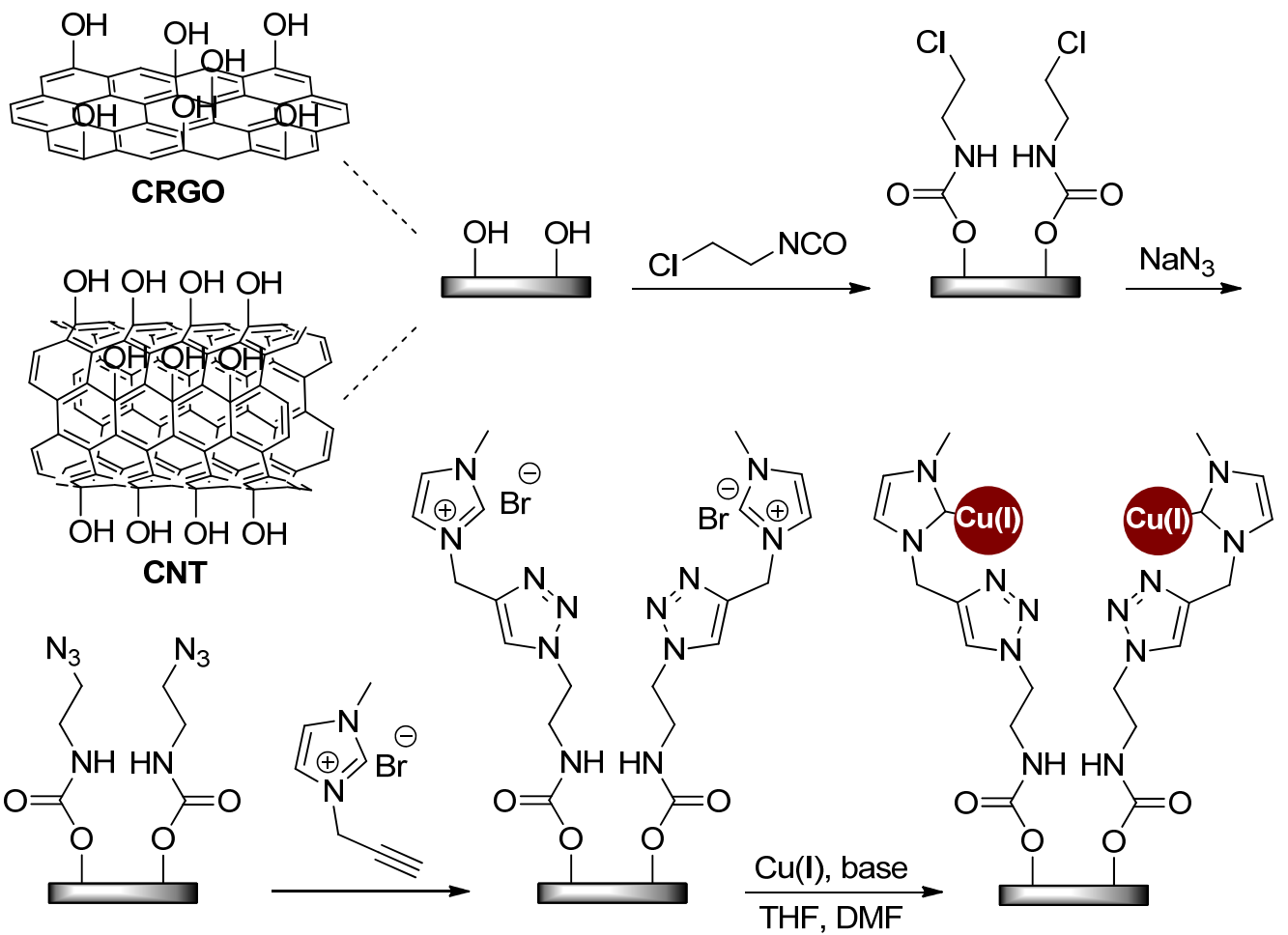

Scheme 62. Schematic route for the immobilization of $\mathrm{N}$-heterocyclic carbene $\mathrm{Cu}(\mathrm{I})$ complexes to graphene and carbon nanotubes (CRGO-Ima-Cu(I) and CNT-Ima-Cu(I), respectively), reported in 2015 by Binder and co-workers.

\subsection{Metal Catalysts Supported on Silica-Anchored Ligands}

Silica is definitely one of the most common inorganic materials used for the preparation of supported catalysts. Besides metal nanoparticles directly deposited onto $\mathrm{SiO}_{2}$ (see Section 2.2), many catalytic systems based on the complexation of suitable metals on silica-anchored ligands have been developed and used in the synthesis of $N$-heterocyclic compounds.

In this context, copper catalysts are among the most investigated systems. In 2013, Díez-González and co-workers described the preparation of a $N$-heterocyclic carbene ligand, the 1,3-diadamantyl-4-hydroxyimidazol-2-ium tetrafluoroborate salt $\left({ }^{\mathrm{OH}} \mathrm{IAd} \cdot \mathrm{HBF}_{4}\right)$, which was then connected to different carriers including silica flakes and silica nanoparticles via a reaction in DMF at $150{ }^{\circ} \mathrm{C}$ in the presence of molecular sieves [153]. The resulting anchored systems, ${ }^{\mathrm{SiFK}} \mathrm{IAd} \cdot \mathrm{HBF}_{4}$ and ${ }^{\mathrm{SiNP}} \mathrm{IAd} \cdot \mathrm{HBF}_{4}$, were treated with $\mathrm{CuI}(\mathrm{NaOBu}-t, \mathrm{THF}$, r.t.) to give the final supported catalysts, which were tested in the $[3+2]$ cycloaddition reactions of azides with terminal alkynes, performed at room temperature and in $\mathrm{H}_{2} \mathrm{O}$ as the solvent. Unfortunately, both ${ }^{\mathrm{SiFK}} \mathrm{IAd} / \mathrm{Cu}(\mathrm{I})$ and ${ }^{\mathrm{SiNP}} \mathrm{IAd} / \mathrm{Cu}(\mathrm{I})$ catalysts showed only modest recyclability, with a clear decrease in their activity from the second cycle; very different behavior (nine runs with no decrease in catalytic activity) was instead observed for the parental system anchored on silica-coated magnetite, whose features will be discussed in detail in Section 4.4 below.

In 2017, Sharghi et al. developed the synthesis of a new amino-functionalized nano silica, which was used to link a copper(II)-2-imino-1,2-diphenylethanol complex, generating a supported copper catalyst (named Cu(II)-ID@Silica by the authors) with high performance in the synthesis of several $N$-heterocycles [154]. Indeed, 1-substituted $1 H$-1,2,3,4-tetrazoles 205 were generated from anilines 204 by three-component reactions with 1.0 equiv. of triethylorthoformate and 1.3 equiv. of $\mathrm{NaN}_{3}$, performed at $65{ }^{\circ} \mathrm{C}$ and in water as the solvent, in the presence of $1 \mathrm{~mol} \%$ of the $\mathrm{Cu}(\mathrm{II})$-ID@Silica catalyst. The same amount 
of catalyst was also able to produce 1,4-disbustituted 1,2,3-1H-triazoles 208 by tandem azidation/[3 + 2] cycloaddition reactions of organic halides or epoxide 206 with $\mathrm{NaN}_{3}$ (1.2 equiv.) and acetylenes 207 (1.0 equiv.), performed in $\mathrm{H}_{2} \mathrm{O}$ at room temperature. The synthesis of 2-aryl-1H-benzo[d] imidazoles 211 from $o$-phenylendiamines 209 (1.2 equiv.) and benzaldehydes 210 (1.0 equiv.), carried out in ethanol as the solvent, required $5 \mathrm{~mol} \%$ of the Cu(II)-ID@Silica catalyst (Scheme 63).

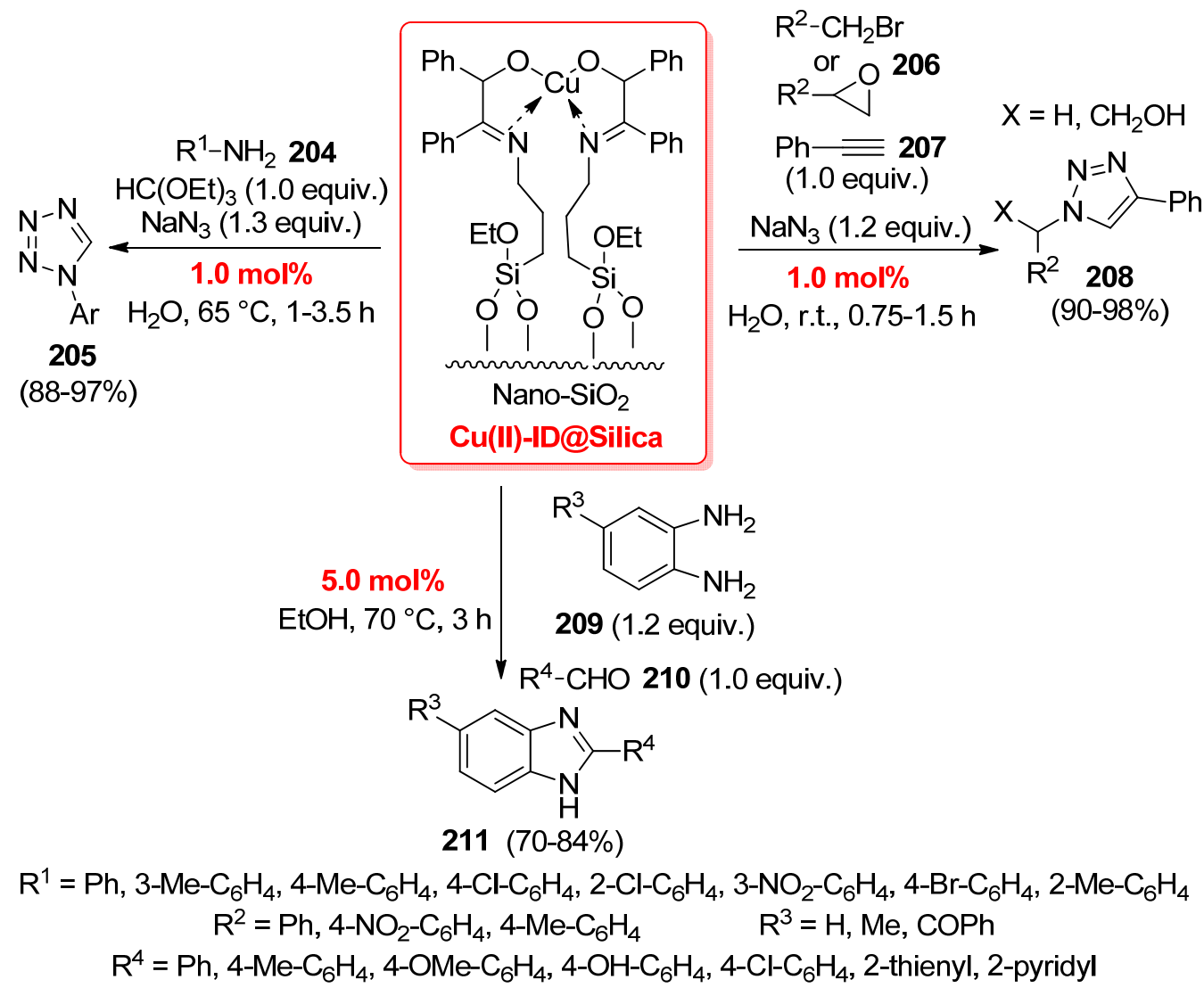

Scheme 63. Synthesis of $1 H$-1,2,3,4-tetrazoles 205, 1,2,3-1H-triazoles 208 and $1 H$-benzo[ $d$ ]imidazoles 211 catalyzed by $\mathrm{Cu}(\mathrm{II})-\mathrm{ID} @$ Silica, reported in 2017 by Sharghi et al.

In 2018, the same group developed a benzimidazole-salen copper(II) complex anchored to a diamino moiety-modified silica (named BS-Cu(II)@SiO $\mathrm{S}_{2}$ by the authors) [155]. Such a catalyst was tested in the synthesis of several functionalized benzimidazole-triazole compounds through a four component $\mathrm{C}-\mathrm{H}$ bond activation/[3 + 2] cycloaddition and through a tandem condensation/cyclization/aromatization sequence. The protocols reported in this study were very convenient from the point of view of Green Chemistry, as they are in full agreement with most of the famous Twelve Principles: reactions were carried out in ethanol as the sustainable solvent (Principle 5: Safer Solvents and Auxiliaries), often at room temperature (Principle 6: Design for Energy Efficiency), using low amounts of a catalyst that is easily recoverable and recyclable up to eight times (Principle 1: Prevention of Waste; Principle 4: Designing Safer Chemicals; Principle 8: Catalysis); furthermore, the choice of tandem, multicomponent reactions for the synthesis of highly functionalized molecules is very convenient from the point of view of the atom economy (Principle 2: Atom Economy). Very recently, they started with the same amino-functionalized silica for the immobilization of a 2-oxoquinoline-3-carbaldehyde Schiff base ligand, which was then treated with copper(II) acetate in refluxing ethanol to give the final supported catalyst, named Cu@QSSi [156]. The catalyst was then used for the synthesis of benzimidazoles 215 by multicomponent reactions of 2-chloroquinoline-3-carbaldehyde 212 with $o$-phenylenediamines 213 and secondary amines 214, carried out at $50{ }^{\circ} \mathrm{C}$ in ethanol as the 
solvent for 2-3 h (Scheme 64). Similarly to BS-Cu(II)@SiO 2 , good recyclability was also observed for the Cu@QSSi catalyst, with only a small decrease in the product yield after the seventh run, although no studies of metal leaching were performed in order to rule out the occurrence of homogeneous copper species during the reactions.

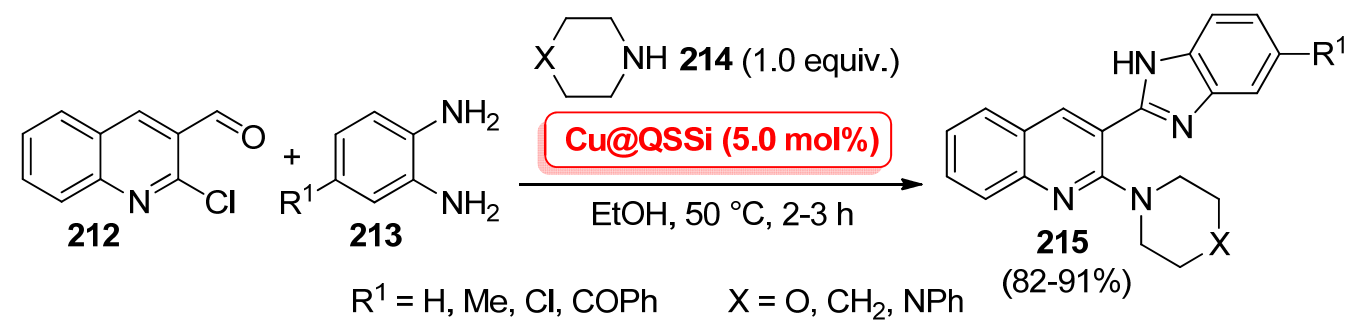

Scheme 64. Synthesis of benzimidazoles 215 by reaction of 2-chloroquinoline-3-carbaldehyde 212 with o-phenylenediamines 213 and secondary amines 214 catalyzed by Cu@QSSi, reported in 2019 by Sharghi et al.

In 2011, Rajagopal and co-workers employed 3-aminopropyltrietoxysilane for the synthesis of $\mathrm{NH}_{2}$-modified silica nanoparticles, which were then treated with poly (ethyleneimine) (PEI)-stabilized copper nanoparticles (in turn obtained by the reaction of $\mathrm{CuCl}_{2}$ with PEI in the presence of $\mathrm{NaBH}_{4}$ ) to give a highly dispersed silica-supported nanocopper catalytic system, named $\mathrm{CuNPs}-\mathrm{PEI} / \mathrm{SiO}_{2}$ [157]. Interestingly, it was applied as a very efficient catalyst in the [3 + 2] azide-alkyne cycloaddition reactions for the synthesis of 1,2,3-1H-triazoles, carried out at room temperature in DMSO as solvent with very short durations (10-60 $\mathrm{min})$, even with a very low amount of the catalyst (i.e., $0.05 \mathrm{~mol} \%$ ). Similar aminopropyl- modified silica nanoparticles $\left(\mathrm{AP}-\mathrm{nSiO}_{2}\right)$ were used in 2013 by Koshropour et al.; in fact, triazine- based dendrimers were covalently anchored to $\mathrm{AP}^{-\mathrm{nSiO}} \mathrm{O}_{2}$, and the resulting material was then treated with $\mathrm{CuCl}_{2}$ in DMF at room temperature for $10 \mathrm{~h}$, to give the final $\mathrm{Cu}(\mathrm{II})-\mathrm{TD} @ \mathrm{nSiO}_{2}$ supported catalyst after filtration and drying [158]. $\mathrm{Cu}(\mathrm{II})-\mathrm{TD} @ \mathrm{nSiO}_{2}$ was tested in the preparation of 2-substituted benzimidazoles 218 by condensation/cyclization of $o$ - phenylenediamines 216 and aldehydes 217, performed under air with $0.3 \mathrm{~mol} \%$ of copper loading, at $50{ }^{\circ} \mathrm{C}$ in ethyl acetate as the solvent (Scheme 65). Moreover, the $\mathrm{Cu}(\mathrm{II})-\mathrm{TD} @ \mathrm{nSiO}_{2}$ catalyst showed good recyclability (up to eight consecutive runs) and a negligible level of metal leaching into the solution, as confirmed by ICP-OES analysis.

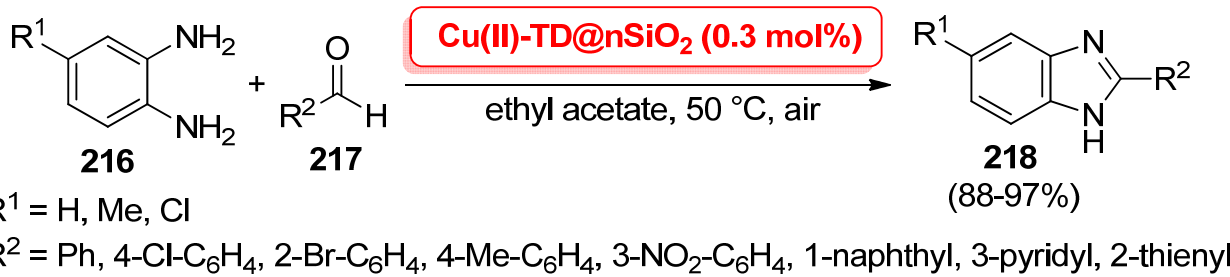

Scheme 65. Synthesis of 2-substituted benzimidazoles 218 by condensation of $o$-phenylenediamines 216 and aldehydes 217 catalyzed by $\mathrm{Cu}(\mathrm{II})-\mathrm{TD} @ \mathrm{nSiO}_{2}$, reported in 2013 by Koshropour et al.

More recently, 3-aminopropyl-functionalized silica was used by Evangelisti, Psaro and co-workers as a hybrid inorganic/organic matrix for the deposition of $\mathrm{Cu}$ nanoparticles prepared by the Metal Vapor Synthesis (MVS) technique [159]. The resulting catalyst (which was named $\mathrm{Cu} / \mathrm{APSiO}_{2}$ by the authors) was characterized by small copper nanoparticles with a mean diameter of $2.5 \mathrm{~nm}$ (HR-TEM analysis) and then tested in the preparation of representative 1,2,3-1H-triazoles by [3 + 2] cycloaddition of benzyl azides with phenylacetylene, performed in both batch and continuous flow conditions (packed-bed reactors). In particular, in this last case, reactions were carried out with $0.5 \mathrm{~mol} \%$ of $\mathrm{Cu} / \mathrm{APSiO}_{2}$, at room temperature in THF with a flow rate of $50 \mu \mathrm{L} / \mathrm{min}$; moreover, packed-bed reactors 
were efficiently reused for five consecutive runs with no loss of activity, while a gradual decrease was observed in the following three runs. Two $\mathrm{SiO}_{2}$-immobilized metal complexes used under continuous flow conditions were also reported by Asensio, Varea et al. in 2017; the $N, N^{\prime}$-bis(4- allyl-2,6-diisopropylphenyl)imidazolium and $N$-adamantyl- $N$-(4allyl-2,6-diisopropylphenyl) imidazolium ligands (IPr and IAdPr, respectively) were first converted into the corresponding triethoxysilyl-substituted derivatives $\operatorname{IPr}_{R}$ and $I_{A d P r} \operatorname{Pr}_{R}$ by platinum-catalyzed hydrosilylation; then, silylated $\mathrm{NHC}-\mathrm{Au}(\mathrm{I})$ complexes $\left[\left(\mathrm{IPr}_{\mathrm{R}}\right) \mathrm{Au}\right] \mathrm{Cl}$ and [(IAdPr $\left.\left.r_{R}\right) \mathrm{Au}\right] \mathrm{Cl}$ were obtained by treatment with $\mathrm{Au}(\mathrm{DMS}) \mathrm{Cl}$ and $\mathrm{K}_{2} \mathrm{CO}_{3}$ in acetone at $60{ }^{\circ} \mathrm{C}$; finally, these complexes were immobilized on activated silica in refluxing toluene using a Dean-Stark apparatus to produce the final supported catalysts $\mathrm{SiO}_{2}-\left[\left(\operatorname{IPr}_{\mathrm{R}}\right) \mathrm{Au}\right] \mathrm{Cl}$ and $\mathrm{SiO}_{2}-\left[\left(\mathrm{IAdPr}_{\mathrm{R}}\right) \mathrm{Au}\right] \mathrm{Cl}$ (Scheme 66) [160]. Such catalysts were employed in several organic transformations, including the cyclization of 1-(ortho-alkynylaryl)urea to generate an indole derivative under batch conditions ( $1 \mathrm{~mol} \%$ of $\mathrm{Au}(\mathrm{I})$, in $\mathrm{EtOH}$ at room temperature), and the cycloisomerization of enynes to give dihydropyrrole and tetrahydropyridine derivatives under continuous flow conditions ( $1 \mathrm{~mol} \%$ of $\mathrm{Au}(\mathrm{I})$, in dry $\mathrm{CH}_{2} \mathrm{Cl}_{2}$ at room temperature).

In 2018, Mohebat, Karimi-Jaberi and co-workers reported the preparation of a very interesting palladium complex immobilized on nano-silica (named n-SiO ${ }_{2}-\mathrm{AP}-\mathrm{CC}-\mathrm{bis}(\mathrm{Amp}$ )Pd by the authors); they first treated nano-silica with 3-aminopropyltrimethoxysilane, in order to obtain a $\mathrm{NH}_{2}$-functionalized nano- $\mathrm{SiO}_{2}$; then, it was treated with cyanuric chloride followed by 2-aminopyridine, giving the anchored ligand; finally, treatment with $\mathrm{Pd}(\mathrm{OAc})_{2}$ afforded the desired n-SiO 2 -AP-CC-bis(Amp)-Pd catalyst [162]. Such a catalyst was applied at low metal loading $(0.2 \mathrm{~mol} \%)$ in the synthesis of 2,3-diarylimidazo[1,2-a]pyridines 224 by the multicomponent reaction of 2-aminopyridine 221 (1.0 equiv.), 2-bromo-1-arylethanones 222 (1.0 equiv.) and aryl-bromides 223 (1.5 equiv.); reactions were carried out in DMA as the solvent, KOAc (3.0 equiv.) as the base and under microwave irradiation, which allowed for a reduction of the reaction time to $45 \mathrm{~min}$ (Scheme 68). Despite that the use of a toxic and harmful reaction medium represents a severe drawback, such a protocol appears quite appealing thanks to the high recyclability of $\mathrm{n}-\mathrm{SiO}_{2}-\mathrm{AP}-\mathrm{CC}$-bis(Amp)-Pd, which has been reused in up to six consecutive runs without a decrease in catalytic activity, in combination with the reduced energy requirements thanks to the use of microwave irradiation.

Moving to palladium catalysts supported on silica-anchored ligands, in 2009, Tyrell et al. reported the immobilization of $\left[\mathrm{PdCl}_{2}(\mathrm{NCPh})_{2}\right]$ to 3-aminopropyl-functionalised $\mathrm{SiO}_{2}$; the resulting material was then tested in the synthesis of indoles derivatives $\mathbf{2 2 0}$ by the heteroannulation of 2-ethynylanylines 219 [161]. Reactions occurred in almost quantitative conversion and high yields when performed with $5 \mathrm{~mol} \%$ of Pd loading, in DMF at $100{ }^{\circ} \mathrm{C}$ for $1 \mathrm{~h}$ (Scheme 67). Although catalyst recyclability has not been directly tested on the heteroannulation reaction, authors actually found that it was recyclable up to four times in the reaction of Sonogashira coupling of terminal alkynes with alkenyl bromides.

Silica was also used for the immobilization of second-generation ruthenium Grubbs catalysts, in order to obtain recoverable catalytic systems, which were successfully applied in the synthesis of $N$-heterocycles by ring-closing olefin metathesis reactions. In this context, Bannwarth et al. reported in 2006 the non-covalent immobilization of a Grubbs-Hoveyda ruthenium catalyst bearing a tris(perfluoroalkyl)silyl chain to a fluorous silica gel; the resulting supported catalyst was applied in preliminary experiments of ring-closing-metathesis of nitrogen-functionalized $\alpha, \omega$-dienes, affording the corresponding $\mathrm{N}$-heterocycles in excellent yields [163]. Interestingly, despite non-covalent immobilization (thanks to the hydrophobic interaction between the perfluorinated tags of both silica and ruthenium complex), the catalyst was successfully recycled for a second run with no loss of activity. A covalent immobilization was instead proposed in 2009 by Grubbs et al.: two triethoxysilyl-functionalized $\mathrm{N}$-heterocyclic carbene ligands were synthesized and used to give the corresponding second-generation ruthenium olefin metathesis catalysts; such complexes were covalently grafted onto silica gel, thus affording the final supported catalysts, which were tested in ring- closing olefin metathesis reactions of several substrates, including $\mathrm{N}, \mathrm{N}$-diallyltosylamide to give the corresponding dihydropyrrole product in excellent 
yields after two hours [164]. A rather similar approach of covalent immobilization was used in 2012 by Pleixats and co-workers, who synthesized a bis-triethoxysilyl-functionalized Heoveyda-Grubbs Ru complex and then anchored to silica by co-gelification with tetraethyl orthosilicate (TEOS) [165]. The resulting anchored ruthenium catalyst was then tested in the preparation of dihydropyrroles 226 by ring-closing-metathesis of $N, N$-diallyltosylamides 225, performed with $2 \mathrm{~mol} \%$ of Ru loading, in dichloromethane at room temperature for 24-48 h, or in toluene at $60-80{ }^{\circ} \mathrm{C}$ for $5-24 \mathrm{~h}$ (Scheme 69). For these substrates, recyclability tests of the supported catalyst were carried out in $\mathrm{CH}_{2} \mathrm{Cl}_{2}$ at room temperature; after the first two runs with no loss of activity, a progressive decrease in the product yield was observed for the third cycle.

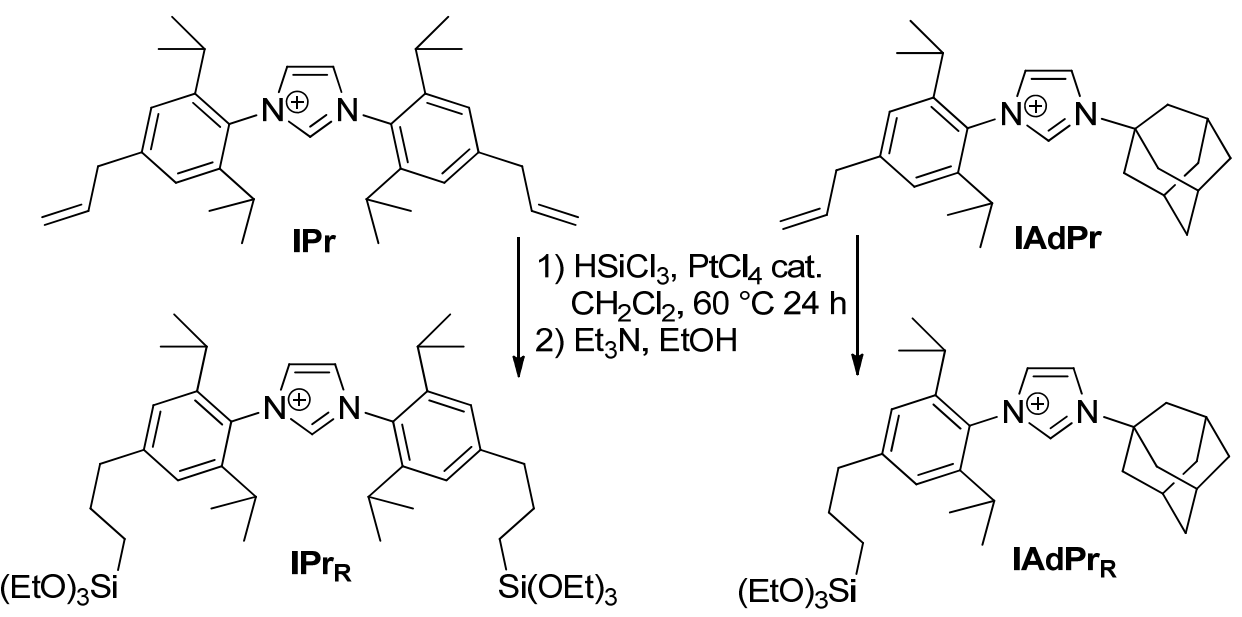

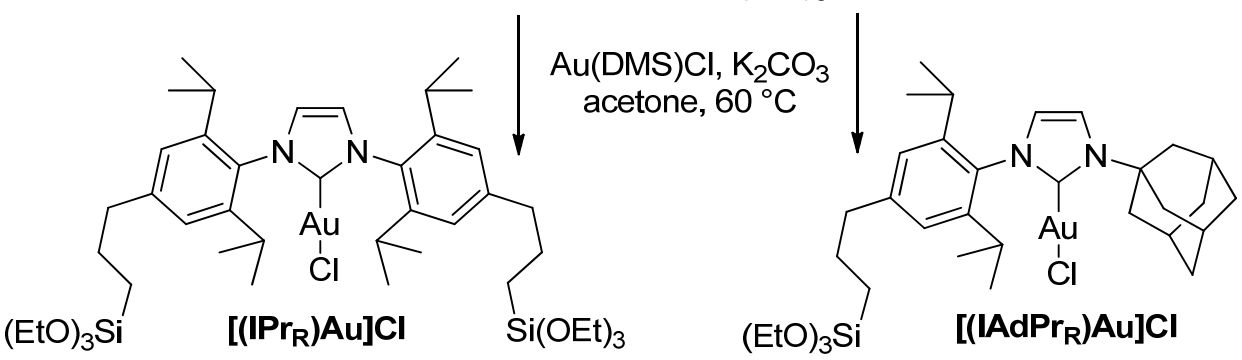

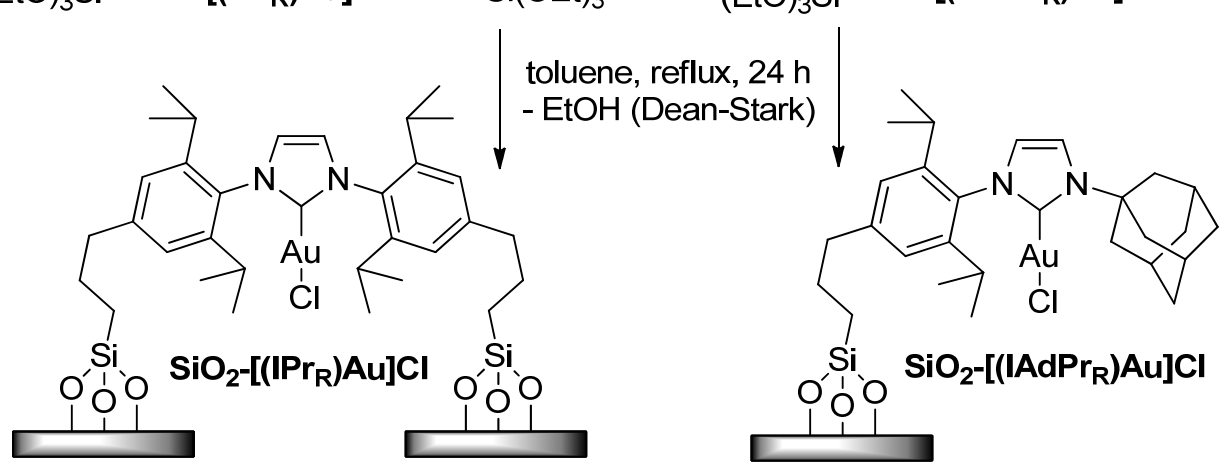

Scheme 66. Schematic route for the immobilization of $\mathrm{Au}(\mathrm{I})$ complexes $\left[\left(\mathrm{IPr}_{\mathrm{R}}\right) \mathrm{Au}\right] \mathrm{Cl}$ and $\left[\left(\mathrm{IAdPr}_{\mathrm{R}}\right) \mathrm{Au}\right] \mathrm{Cl}$ on activated silica, reported in 2017 by Asensio, Varea et al.

The 3-aminopropyl-functionalised silica was also used as an organic-inorganic hybrid material for the immobilization of a zirconium(IV) complex, as reported in 2011 by Sharma et al.; such a supported catalyst, named Zr-CAP-SG, showed remarkable activity in the one-pot condensation of 1,2-diketones with ortho-phenylenediamines or (hetero)aryl aldehydes affording quinoxalines and 2,4,5-trisubstituted imidazoles, respectively [166]. The optimized reaction conditions were very appealing from the point of view of Green Chemistry, thanks to the use of sustainable solvents (ethanol or acetonitrile), the need for 
room temperature and short reaction times (i.e., limited energy requirement) and the use of a highly recyclable catalytic system (up to five runs with no loss of activity).

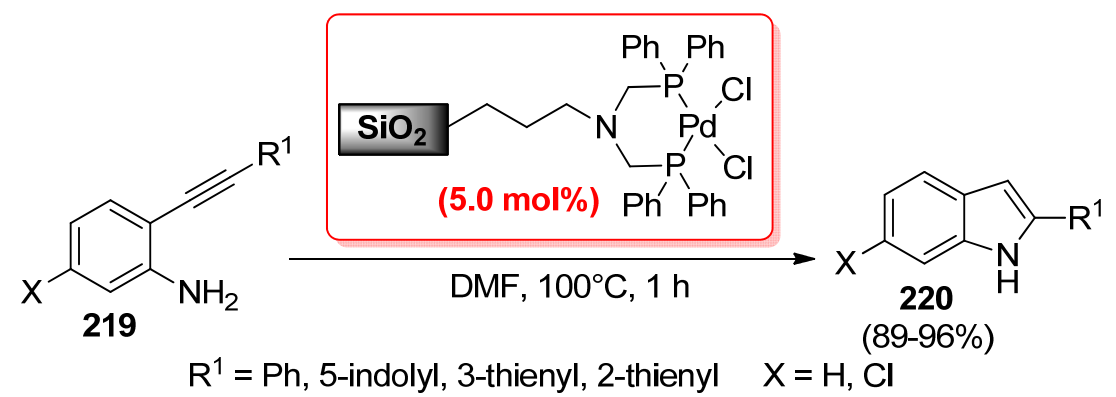

Scheme 67. Synthesis of indoles derivatives 220 by heteroannulation of 2-ethynylanylines 219 catalyzed by $\left[\mathrm{PdCl}_{2}(\mathrm{NCPh})_{2}\right]$ immobilized to 3-aminopropyl-functionalised $\mathrm{SiO}_{2}$, reported in 2009 by Tyrell et al.

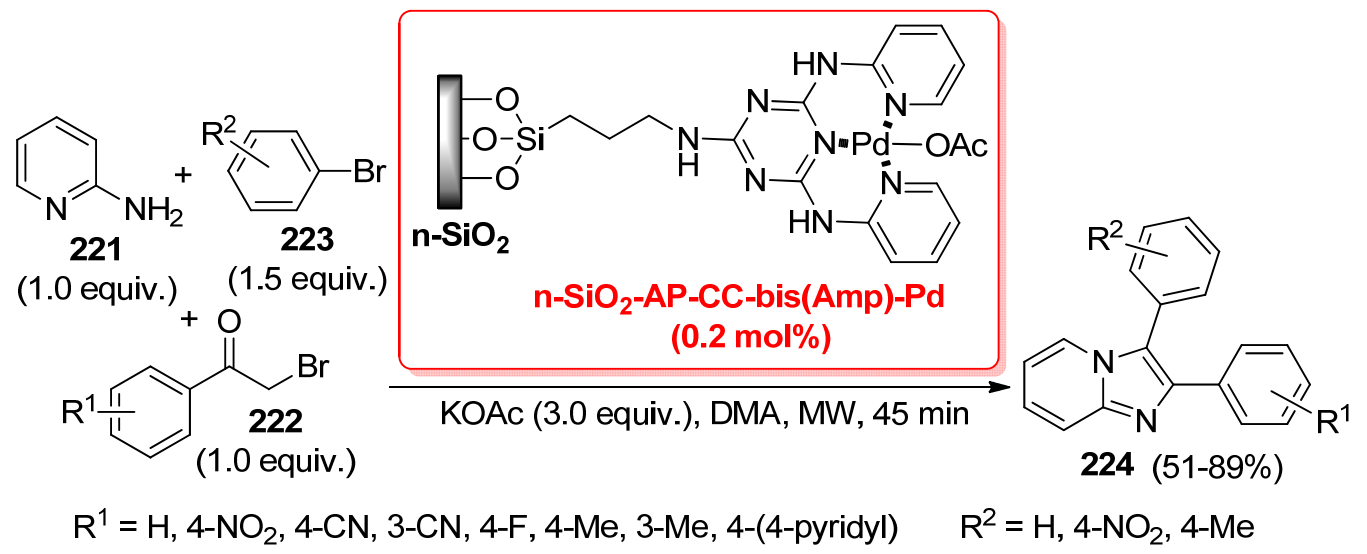

Scheme 68. Synthesis of imidazo[1,2-a]pyridines 224 by multicomponent reaction of 2-aminopyridine 221, 2-bromo-1-arylethanones 222 and aryl-bromides 223 catalyzed by n-SiO ${ }_{2}-\mathrm{AP}-\mathrm{CC}$-bis(Amp)-Pd, reported in 2018 by Mohebat, Karimi-Jaberi and co-workers.

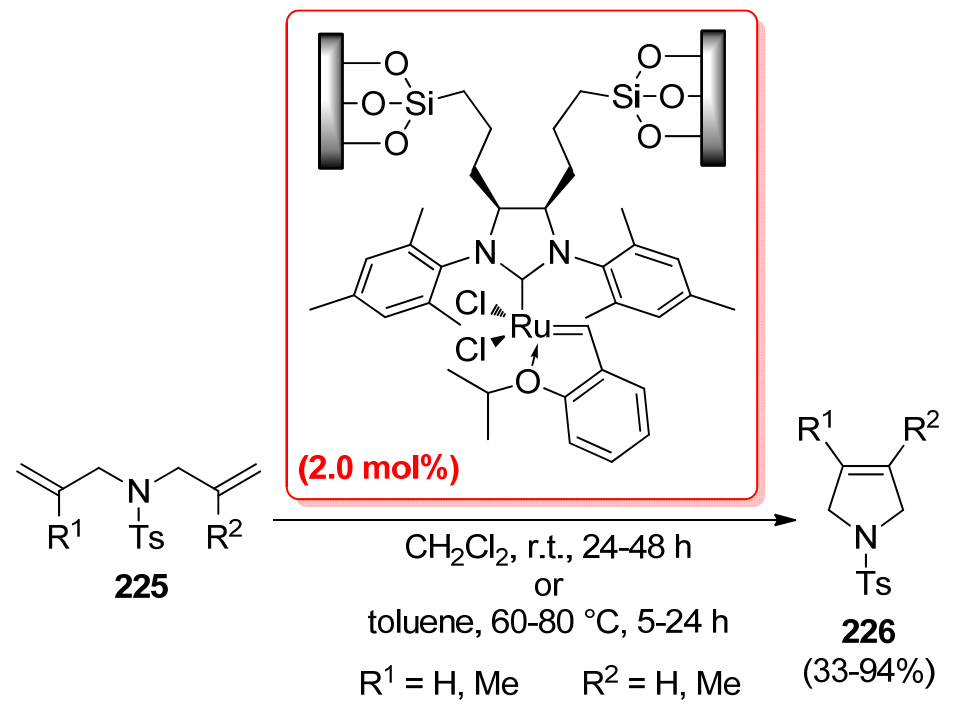

Scheme 69. Synthesis of dihydropyrroles 226 by ring-closing-metathesis of $N, N$-diallyltosylamides 225 catalyzed by Heoveyda-Grubbs ruthenium complex immobilized on silica, reported in 2012 by Pleixats and co-workers. 
As already mentioned in Section 2.2, a plethora of ordered mesoporous silica has recently found huge interest as a support for metal catalysts. Therefore, it should be not surprising that these materials have successfully been used for the immobilization of organic ligands, suitable for the complexation of various metals to give valuable supported catalysts used in the synthesis of $N$-heterocyclic compounds.

The Santa Barbara Amorphous-15 (SBA-15) mesoporous silica possesses hexagonal arrays of large pores (3-30 nm), with a large number of hydroxyls in the internal surfaces; moreover, it is characterized by high thermal and physical stability, making it particularly suitable as a support for metal catalysts. In 2017, Hajjami and co-workers described the synthesis of a $\mathrm{Cu}(\mathrm{I})-1,3$-dimethylbarbituric acid complex immobilized on mesoporous SBA15 silica. First, SBA-15 was treated with 3-aminopropyltriethoxysilane in order to obtain the corresponding amino-functionalized material (SBA-15- $n-\mathrm{Pr}-\mathrm{NH}_{2}$ ); then, such material was added to a solution of 1,3-dimethylbarbituric acid in ethanol and refluxed for $48 \mathrm{~h}$; finally, the SBA-15-anchored ligand was added to a solution of $\mathrm{CuCl}$ in acetonitrile and stirred for $24 \mathrm{~h}$ at room temperature, giving the final SBA-15-DMBA-Cu(I) catalyst [167]. Such a catalyst was then applied at low copper loading $(0.7 \mathrm{~mol} \%)$ to the one-pot synthesis of trisubstituted imidazoles 227 and tetrasubstituted imidazoles $\mathbf{2 2 8}$ under solvent-free conditions at $100{ }^{\circ} \mathrm{C}$; the expected products were obtained in excellent yields and the SBA-15-DMBA-Cu(I) catalyst could be easily isolated and reused up to five times without a loss of activity (Scheme 70).

A copper(II) complex anchored to SBA-15 was instead prepared in 2015 by Bardajee and co-workers; they employed the hexaminemacrobicyclic ligand DiAmSar to encapsulate $\mathrm{Cu}(\mathrm{II})$ ions inside the macrocyclic cage, affording $\mathrm{Cu}(\mathrm{II})$-DiAmSar species; the final heterogeneous catalyst $\mathrm{Cu}(\mathrm{II})$-DiAmSar/SBA-15 was then generated by reaction of the $-\mathrm{NH}_{2}$ groups of the ligand with (3-chloropropyl)trimethoxysilane, followed by the covalent immobilization onto SBA-15 through the trimethoxysilane moiety [168]. The obtained material was successfully tested as a supported catalyst for the synthesis of pyridopyrazine and quinoxaline derivatives 231 by condensation of 1,2-diketones 229 with 1,2-diamines 230. Interestingly, reactions were carried out with a very low copper loading (only $0.008 \mathrm{~mol} \%$ ), under solvent-free conditions at $100{ }^{\circ} \mathrm{C}$ for 5-90 min (Scheme 71). The protocol appears very interesting from the point of view of the Twelve Principles of Green Chemistry, thanks to the absence of a reaction medium (Principle 5: Safer Solvents and Auxiliaries) and very low amounts of a recoverable and recyclable catalyst (Principle 1: Prevention of Waste; Principle 4: Designing Safer Chemicals; Principle 8: Catalysis). A similar approach for the synthesis of quinoxalines and pyridopyrazine derivatives was reported by the same research group using iron [169], zirconium [170] and palladium [171] Schiff-base complexes grafted on SBA-15: each of them could be successfully used in water as the solvent with a low metal amount $(0.1-0.5 \mathrm{~mol} \%)$.

In 2010, Djakovitch, Dufaud et al. reported the preparation of four different palladium(II) complexes of the general formula $\mathrm{PdCl}_{2} \mathrm{~L}_{2}$, with $\mathrm{L}=$ phosphine or nitrile ligand bearing a triethoxysilyl moiety, which were then covalently anchored to SBA-15 [172]. The performances of the four supported catalysts were compared in a representative example of Larock synthesis of indole 234 between 2-iodoaniline 232 and triethyl(phenylethynyl)silane 233, performed in DMF at $120^{\circ} \mathrm{C}$ with $1 \mathrm{~mol} \%$ of palladium, in the presence of 3.0 equiv. of $\mathrm{Na}_{2} \mathrm{CO}_{3}$. Among the four catalysts, the hybrid system in Scheme 72 showed the highest catalytic activity, but several mechanism investigations revealed its behavior as a reservoir of soluble active metal particles in the solution, responsible for substantial deactivation upon recycling. 


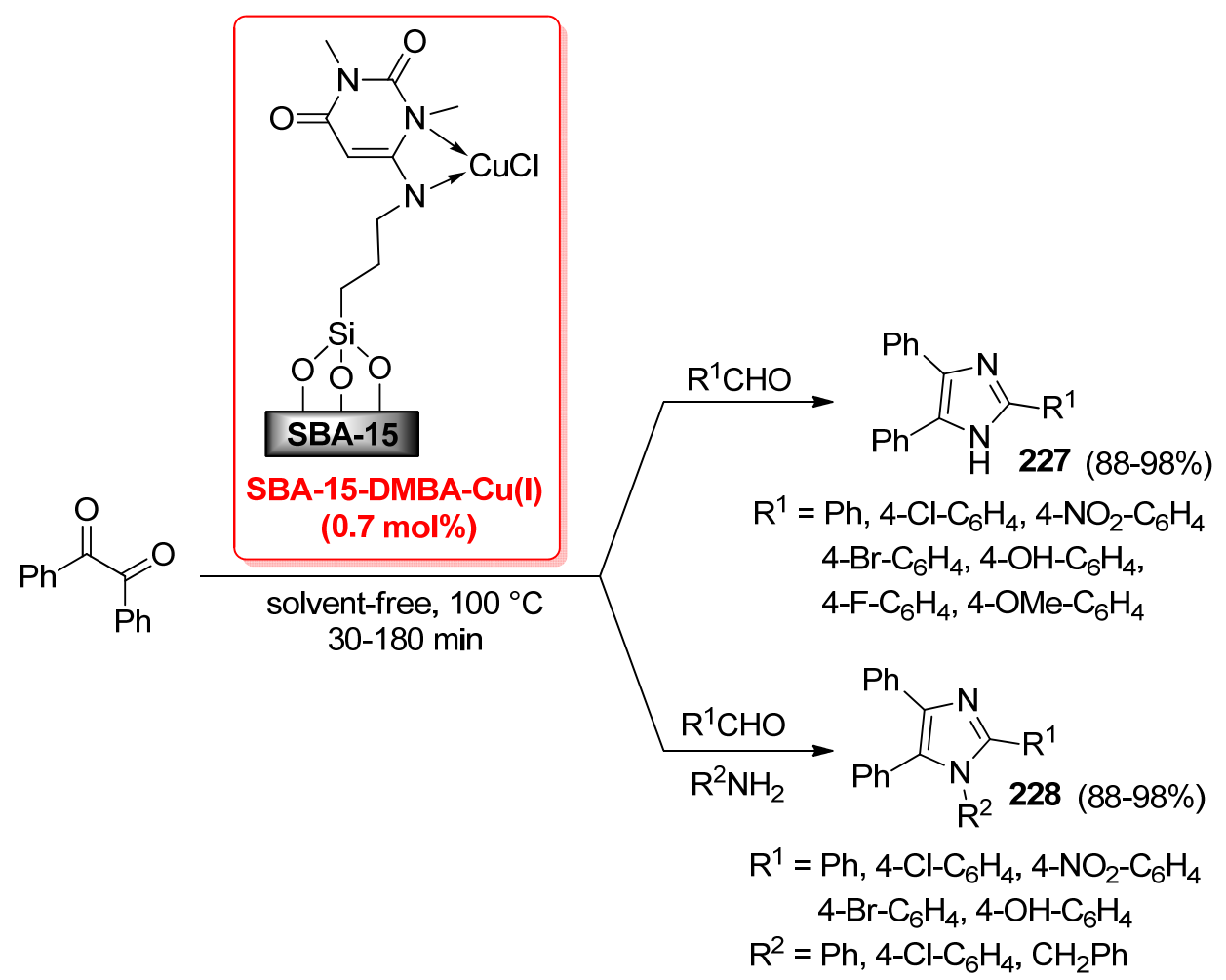

Scheme 70. One-pot synthesis of trisubstituted and tetrasubstituted imidazoles 227-228 catalyzed by SBA-15-DMBA-Cu(I), reported in 2017 by Hajjami and co-workers.

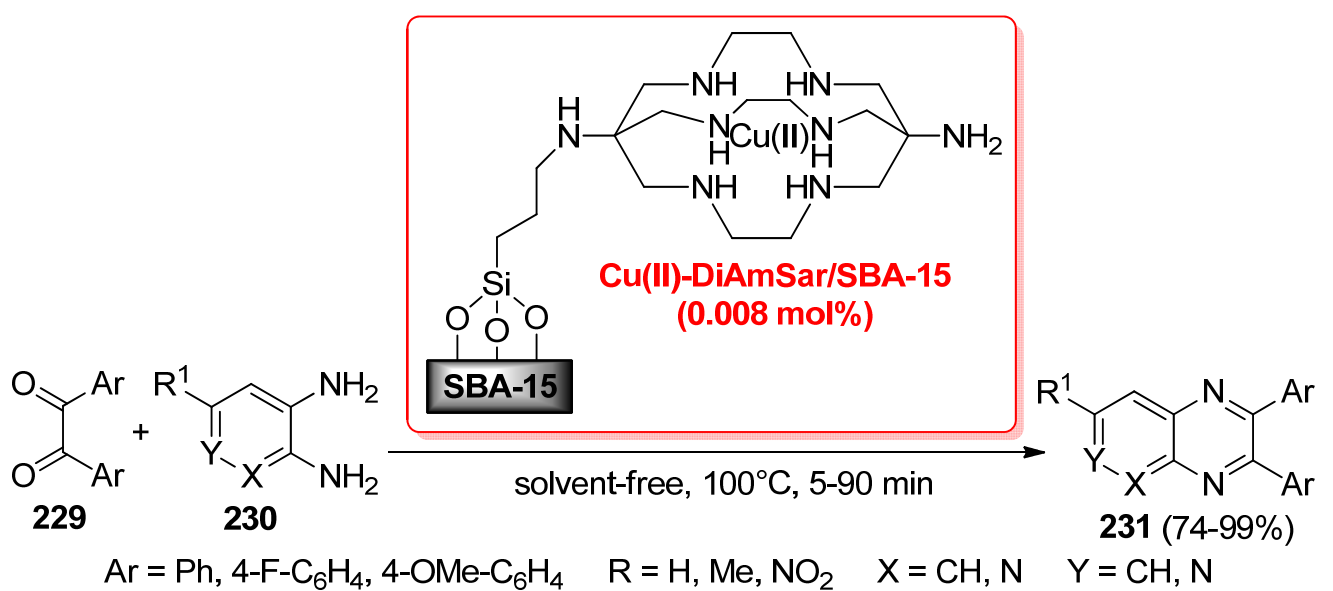

Scheme 71. Synthesis of pyridopyrazines and quinoxalines 231 by condensation of 1,2-diketones 229 with 1,2-diamines 230 catalyzed by Cu(II)-DiAmSar/SBA-15, reported in 2015 by Bardajee et al.

An ammonium tagged Hoveyda-type ruthenium catalyst bearing a sterically enlarged $N$-heterocyclic carbene ligand was synthesized in 2015 by Skowerski and co-workers and then easily immobilized on mesoporous SBA-15 silica by non-covalent interactions [173]. The resulting supported material was then tested in the ring-closing metathesis of several nitrogen-containing $\alpha, \omega$-dienes, to give the corresponding $N$-heterocyclic products; such a catalyst showed exceptionally high recyclability, with an average conversion of $66 \%$ after 23 cycles, which corresponded to a cumulative TON of 15180. 


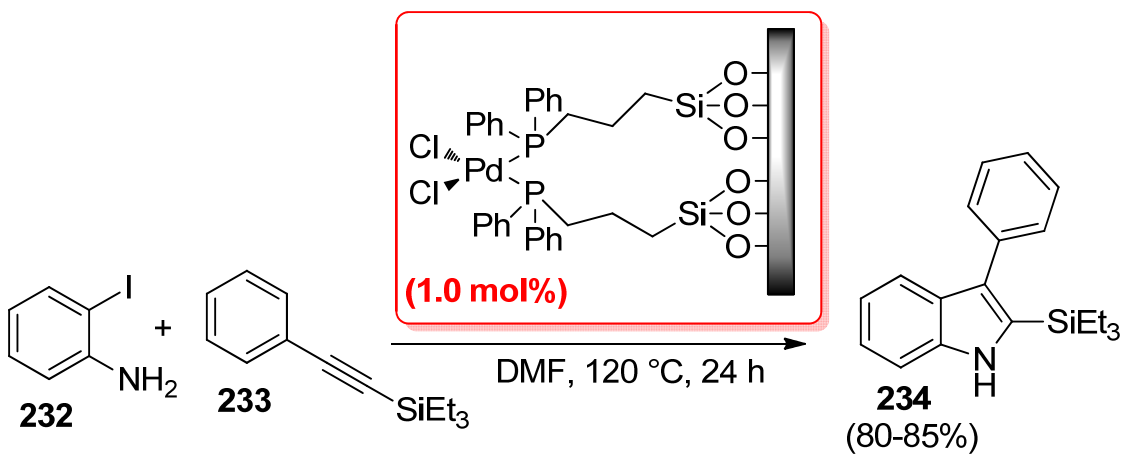

Scheme 72. Larock synthesis of indole 234 from 2-iodoaniline 232 and triethyl(phenylethynyl)silane 233 catalyzed by SBA-15-anchored $\mathrm{PdCl}_{2} \mathrm{~L}_{2}$ complex, reported in 2010 by Djakovitch, Dufaud et al.

The Mobil Composition of Matter No. 41 (MCM-41) is a mesoporous material characterized by high surface area, large pore volume and uniform pore size. In 2017, Cai et al. described the synthesis of a heterogeneous gold catalyst (named MCM-41-PPh $-\mathrm{AuNTf}_{2}$ ) by immobilization of a phosphine gold(I) complex onto MCM-41 [174]. This catalyst was then applied (at $5 \mathrm{~mol} \%$ of Ru loading) in the cascade of annulation reactions of (hetero)aryl aldehydes 235 with propargylamine 236, performed in the presence of $\mathrm{H}_{2} \mathrm{O}$ (5.0 equiv.) as an additive, 1,2-dichloroethane as the solvent at $40{ }^{\circ} \mathrm{C}$ for $48 \mathrm{~h}$, to afford pyrazines 237 in good to excellent yields (Scheme 73). Moreover, MCM-41-PPh $3-\mathrm{AuNTf}_{2}$ could be easily recovered and reused for eight consecutive runs with no loss of catalytic activity; this was confirmed by ICP-AES analysis, revealing the absence of gold leaching from the heterogeneous catalyst into the solution.

In a following study, the same authors developed a very similar $\mathrm{Au}(\mathrm{I})$-phosphine complex immobilized onto MCM-41, called MCM-41-PPh $-\mathrm{AuCl}$, which was applied in the annulations of 2-aminopyridines 238 with alkynyl aldehydes 239 to give the corresponding 3-acylimidazo[1,2-a]pyridines 240 [175]. Reactions were carried out with $3 \mathrm{~mol} \%$ of $\mathrm{Au}$ loading, $3 \mathrm{~mol} \%$ of $\mathrm{AgSbF}_{6}$ as co-catalyst and $5 \mathrm{~mol} \%$ of acetic acid as additive, in $\mathrm{CH}_{2} \mathrm{Cl}_{2}$ as the solvent at room temperature for $12 \mathrm{~h}$ (Scheme 74). Although MCM-41- $\mathrm{PPh}_{3}-\mathrm{AuCl}$ showed recyclability very similar to the abovementioned $\mathrm{MCM}-41-\mathrm{PPh}_{3}-\mathrm{AuNTf}_{2}$ (eight consecutive cycles without a decrease in the product yields), the presence of homogeneous $\mathrm{AgSbF}_{6}$ as the co-catalyst represents the main issue of this protocol, since silver species could contaminate the final imidazo[1,2-a]pyridine products.

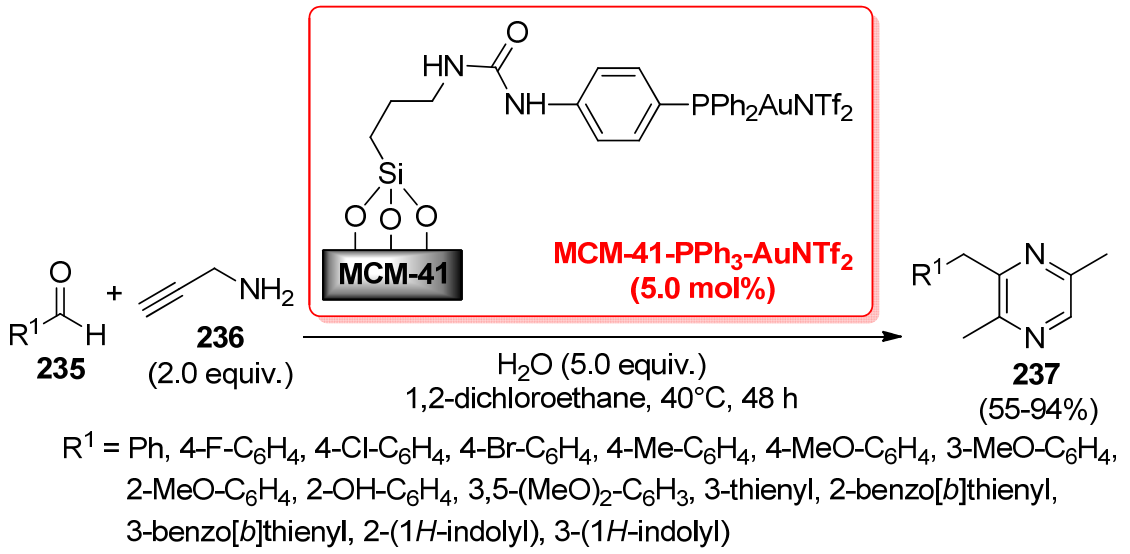

Scheme 73. Synthesis of pyrazines 237 by cascade annulation reactions of (hetero)aryl aldehydes 235 with propargylamine 236 catalyzed by MCM-41-PPh $3-\mathrm{AuNTf}_{2}$, reported in 2017 by Cai et al. 


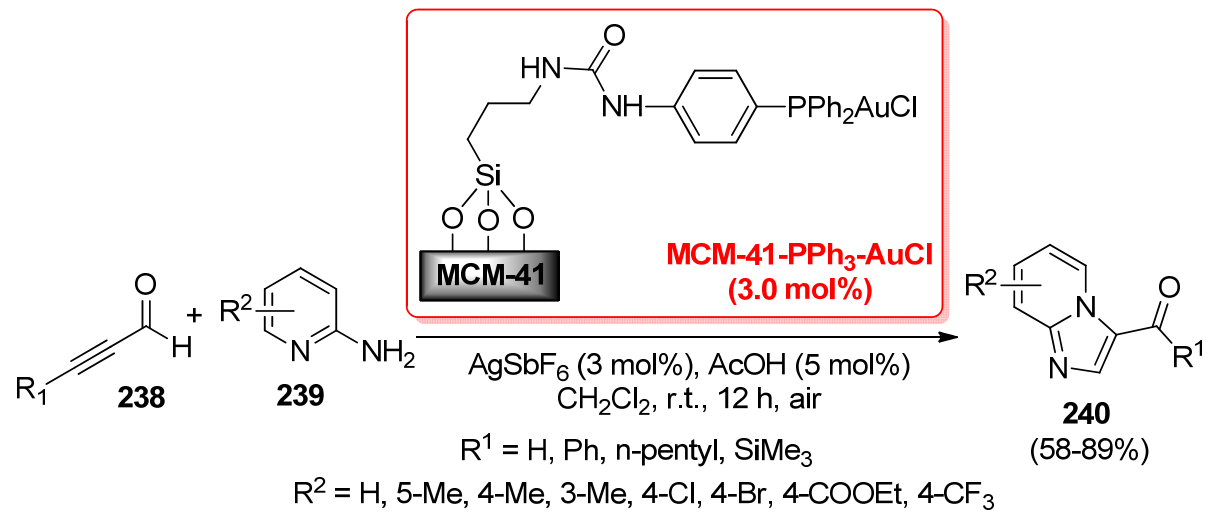

Scheme 74. Synthesis of 3-acylimidazo[1,2-a]pyridines 240 by annulations of 2-aminopyridines 238 with alkynyl aldehydes 239 catalyzed by $\mathrm{MCM}-41-\mathrm{PPh}_{3}-\mathrm{AuCl}$, reported in 2018 by Cai et al.

More recently, Cai et al. moved their attention to copper species anchored onto MCM41 mesoporous silica. In more detail, a MCM-41-anchored bidentate 2-aminoethylamino $\mathrm{Cu}$ (II) complex (named MCM-41-2N-Cu(OAc) $)_{2}$ ) was synthesized and then tested in the oxidative cyclization of 2-pyridine ketone hydrazones 241 [176]. Reactions were carried out in ethyl acetate as the solvent, with $5 \mathrm{~mol} \%$ of $\mathrm{Cu}$ loading, at room temperature for 30-180 min, affording a variety of 1,2,3-triazolopyridines 243 in good to excellent yields (Scheme 75a). However, considering that hydrazones could be easily obtained from the corresponding ketones by the reaction with hydrazine, the authors also developed a protocol for the preparation of 1,2,3-triazolopyridines 243 directly from 2-acylpyridines 242 , involving a preliminary treatment with hydrazine monohydrate (1.5 equiv.) in the presence of $\mathrm{AcOH}(10 \mathrm{~mol} \%)$, in refluxing $\mathrm{EtOH}$ for $6 \mathrm{~h}$, followed by the oxidative cyclization step in $\mathrm{EtOAc} / \mathrm{EtOH}(5: 1 \mathrm{v} / \mathrm{v})$ in the presence of MCM-41-2N-Cu(OAc) $(5 \mathrm{~mol} \%)$ at room temperature for 30-180 min (Scheme 75b).

A $N$-heterocyclic carbene- $\mathrm{Cu}(\mathrm{I})$ species anchored to MCM- 41 was described by Oro and co-workers, who then tested it as a recoverable catalyst for the synthesis of a functionalized 1,2,3-1H-triazole by [3 + 2] cycloaddition of benzylazide and phenylacetylene [177]. In 2020, Nikoorasm et al. reported the preparation of 5-substituted $1 \mathrm{H}$-tetrazoles 245 via the reaction of benzonitriles 244 with sodium azide catalyzed by copper(II) or nickel(II) complexed to a cytosine ligand anchored to mesoporous MCM-41 silica [178]. In particular, reactions were carried out at $120^{\circ} \mathrm{C}$ in PEG-400 as the solvent for 15-240 min (Scheme 76); between the two catalysts, $\mathrm{Ni}(\mathrm{II})$-cytosine-MCM-41 resulted as the most effective, yielding $1 H$-tetrazole products in higher yields with a lower metal loading compared to $\mathrm{Cu}$ (II)-cytosine-MCM41 (i.e., $0.1 \mathrm{~mol} \%$ of $\mathrm{Ni}$ vs. $3 \mathrm{~mol} \%$ of $\mathrm{Cu}$ ). However, both catalytic systems exhibited comparable reusability: $\mathrm{Ni}(\mathrm{II})$-cytosine-MCM- 41 and $\mathrm{Cu}(\mathrm{II})$-cytosine-MCM-41 were used for six consecutive runs with no significant loss of activity; the result was further confirmed by SEM analysis of both catalysts after the sixth run of recycling, which showed that the size and morphology were very similar to the corresponding fresh catalysts. ICP-AAS analysis of the reaction mixture after catalyst removal revealed neither copper nor nickel leaching into the solution, thus confirming their merely heterogeneous nature. These features make both catalysts very appealing in terms of green and sustainable chemistry.

Other mesoporous silica materials have been only occasionally applied as a support for the immobilization of metal complexes to be used as catalysts for the synthesis of nitrogen-containing heterocycles. Córdova and co-workers reported the use of palladium nanoparticles immobilized on 3-aminopropyl-functionalized siliceous mesocellular foam (named $\mathrm{Pd}(0)-\mathrm{AmP}-\mathrm{MCF}$ ) in combination with a chiral diphenylprolinol derivative as an organocatalyst, for the enantioselective tandem reaction of $\alpha, \beta$-unsaturated aldehydes with proparylamine to give the corresponding chiral dihydropyrroles with high yields and good enantiomeric excess (up to 98\%) [179]. In a following study, the authors used the same combined catalytic system for the preparation of chiral aziridines [180]. In both studies, the 
authors found high recyclability for the supported palladium catalyst, as it was used for eight consecutive cycles with no evident loss of its catalytic activity.

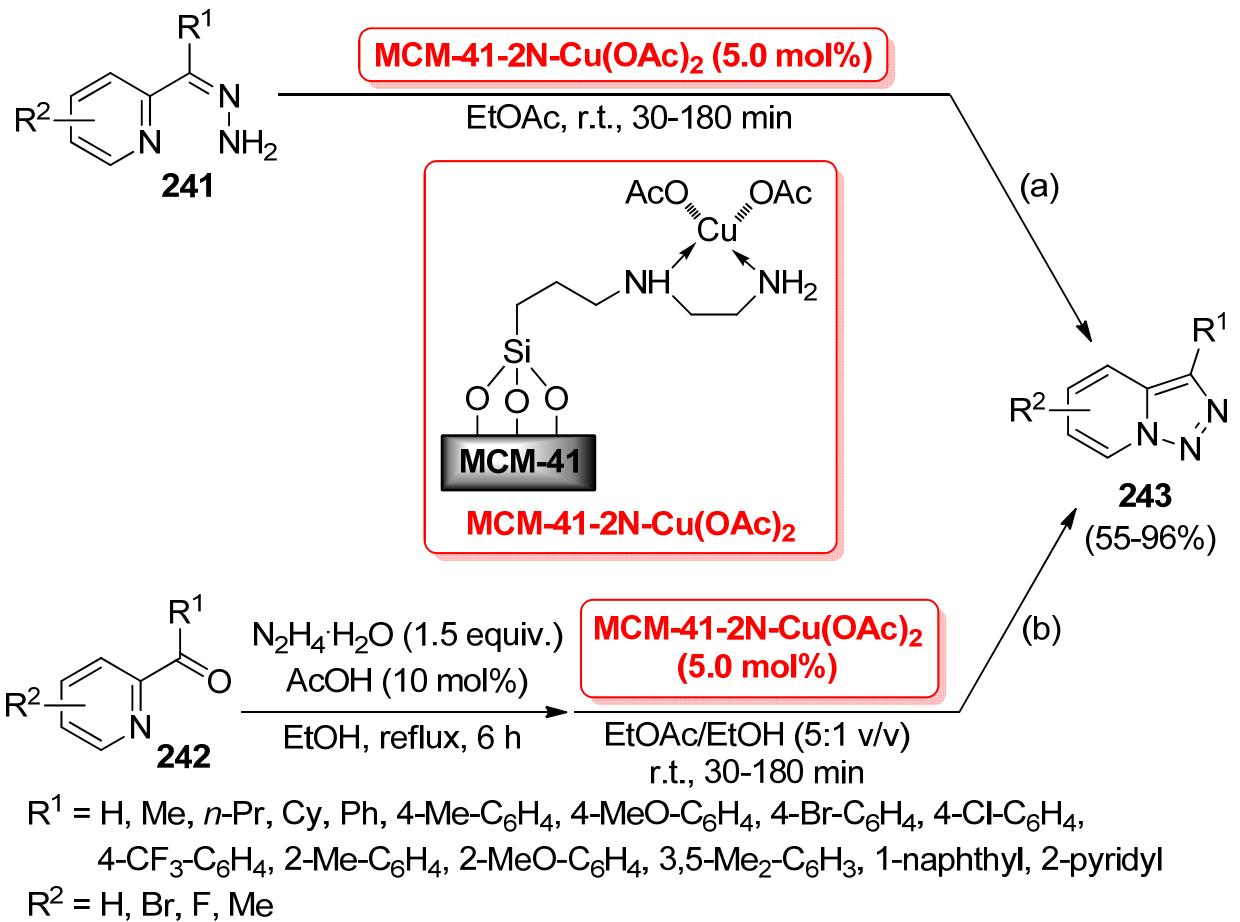

Scheme 75. Synthesis of 1,2,3-triazolopyridines 243 by (a) oxidative cyclization of 2-pyridine ketone hydrazones 241; (b) tandem hydrazine synthesis/oxidative cyclization of 2-acylpyridines 242 catalyzed by MCM-41-2N-Cu(OAc) $)_{2}$, reported in 2019 by Cai et al.

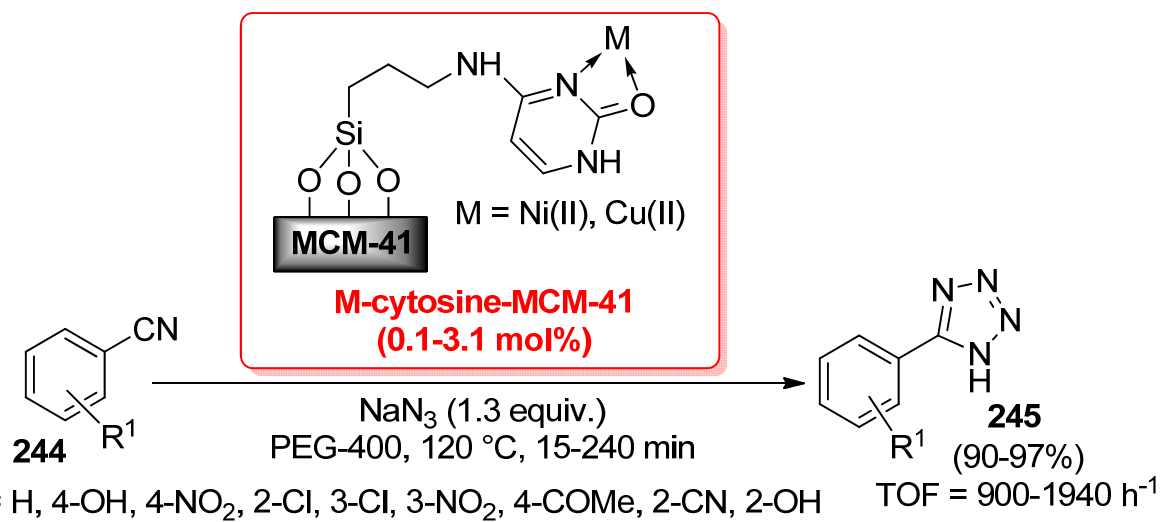

Scheme 76. Synthesis of 5-substituted $1 H$-tetrazoles 245 by reaction of benzonitriles 244 with sodium azide catalyzed by $\mathrm{Cu}(\mathrm{II})$-cytosine-MCM-41 and Ni(II)-cytosine-MCM-41, reported in 2020 by Nikoorasm et al.

\subsection{Metal Catalysts Supported on Metal Oxides or Minerals-Anchored Ligands}

The development of metal catalysts supported on metal oxides-anchored ligands is a topic of recent interest: only in the last six years have some investigations involving the use of iron oxides (also in the form of ferrites) been reported in the context of the preparation of $N$-heterocycles.

The first study in this context was reported in 2015 by Zhang et al., whereby they functionalized nickel ferrite $\left(\mathrm{NiFe}_{2} \mathrm{O}_{4}\right)$ nanoparticles with $L$-glutamate units through the $-\mathrm{COOH}$ of the side chains, and the resulting $\mathrm{NiFe}_{2} \mathrm{O}_{4}$-glutamate material was treated with $\mathrm{Cu}(\mathrm{OAc})_{2}$ in combination with reducing agents (hydrazine and $\mathrm{NaBH}_{4}$ ) to give 
the final $\mathrm{NiFe}_{2} \mathrm{O}_{4}$-glutamate-Cu(0) supported catalyst [181]. Such a catalyst exhibited excellent performance in the tandem $\mathrm{S}_{\mathrm{N}} 2 /[3+2]$ cycloaddition reaction of terminal alkynes 246 and sodium azide with (a) epoxides 247 for the synthesis of $\beta$-hydroxy-1,2,3-1Htriazoles 248 and (b) benzyl chlorides 249 or arylboronic acids 250 for the synthesis of 1,4-disubstituted 1,2,3-1H-triazoles 251. The reactions were carried out with $1.0-5.0 \mathrm{~mol} \%$ of the $\mathrm{NiFe}_{2} \mathrm{O}_{4}$-glutamate- $\mathrm{Cu}(0)$ catalyst, at room temperature in $\mathrm{H}_{2} \mathrm{O}$ as the solvent for 1.5-8.5 h (Scheme 77). Interestingly, $\mathrm{NiFe}_{2} \mathrm{O}_{4}$-glutamate- $\mathrm{Cu}(0)$ showed high reusability (up to 10 times), although no studies were carried out in order to rule out copper leaching into the solution.

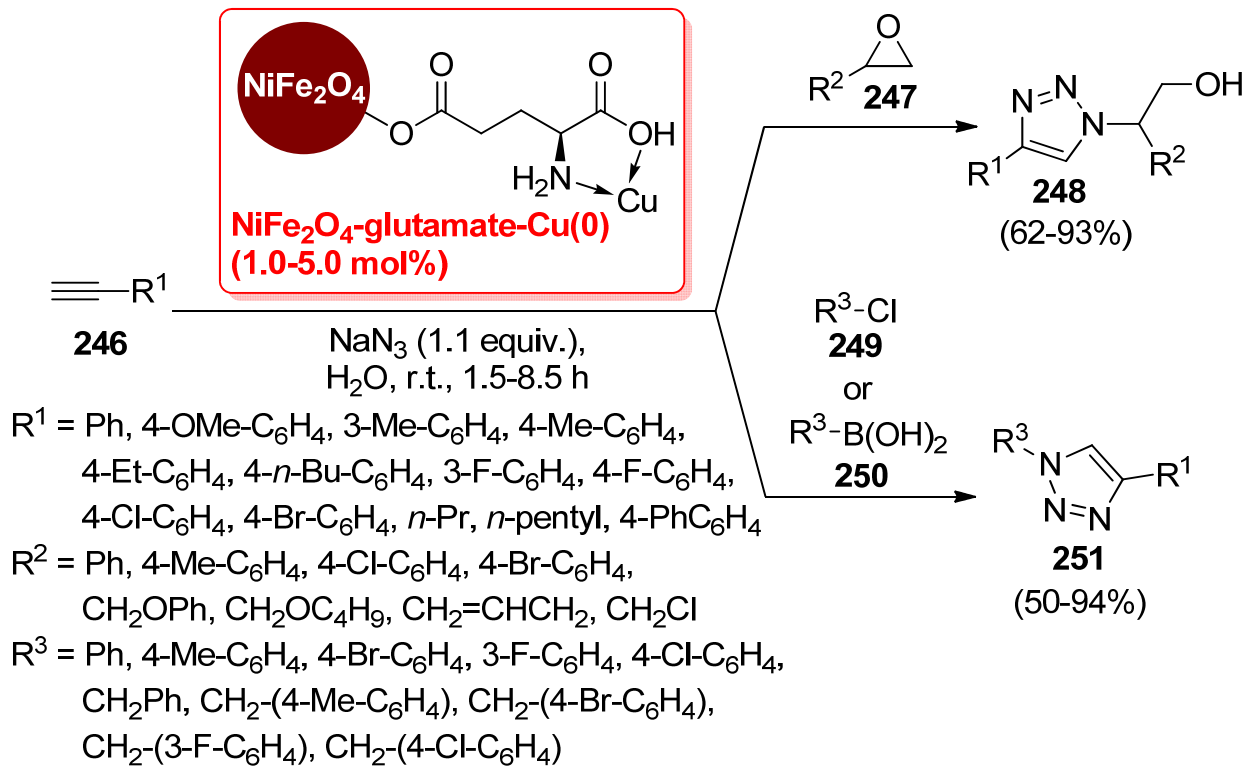

Scheme 77. Synthesis of 1,2,3-1H-triazoles 248 and 251 by tandem $\mathrm{S}_{\mathrm{N}} 2 /[3+2]$ cycloaddition reaction of terminal alkynes 246 catalyzed by $\mathrm{NiFe}_{2} \mathrm{O}_{4}$-glutamate- $\mathrm{Cu}(0)$, reported in 2015 by Zhang et al.

In 2016, Guorbani-Choghamarani and co-workers instead used a different $\alpha$-amino acid, L-hystidine, as a ligand for $\mathrm{Cu}$ species to be anchored onto iron oxides: magnetite $\left(\mathrm{Fe}_{3} \mathrm{O}_{4}\right)$ was functionalized with the amino group and then used for the covalent immobilization of the imidazole units of L-hystidines, able to coordinate copper(II) by treatment with $\mathrm{CuCl}_{2}$ [182]. The resulting catalyst, named $\mathrm{Cu}(\mathrm{II})-\mathrm{L}-\mathrm{HIS} @ \mathrm{Fe}_{3} \mathrm{O}_{4}$ by the authors, was evaluated in a very appealing synthesis of 2-amino-3,5-dicarbonitrile-6-thio-pyridines 254 by the multicomponent reaction of benzaldehydes 252, thiophenols 253 and malononitrile (2.0 equiv.), carried out in $\mathrm{H}_{2} \mathrm{O}$ as the solvent, at $80^{\circ} \mathrm{C}$ for $60 \mathrm{~min}$ with $0.48 \mathrm{~mol} \%$ of copper loading (Scheme 78). Such a protocol fits well with most of the Twelve Principles of Green Chemistry, thanks to the use of $\mathrm{H}_{2} \mathrm{O}$ as a highly sustainable reaction medium, the need for moderate energy requirements with the use of thermal heating for a very short time and the application of a highly recyclable (up to six times) heterogeneous metal catalyst.

Copper(II) complexed to an $\mathrm{Fe}_{3} \mathrm{O}_{4}$-functionalized 5,10-dihydropyrido[2,3-b] quinoxaline-7,8-diol ligand was instead developed in 2018 by Habibi and co-workers for the synthesis of 1-substituted $1 H$-tetrazoles by a one-pot, three-component reaction of anilines with sodium azide and triethylorthoformate [183]. Such a catalyst, named $\mathrm{Fe}_{3} \mathrm{O}_{4} @$ Quinindiol@ $\mathrm{Cu}$ by the authors, worked well with anilines bearing both electrondonating $\left(-\mathrm{CH}_{3},-\mathrm{OCH}_{3}\right)$ or electron-withdrawing functional groups $\left(-\mathrm{Cl},-\mathrm{Br},-\mathrm{NO}_{2}\right.$, $\left.-\mathrm{COCH}_{3},-\mathrm{CF}_{3}\right)$, affording the desired $\mathrm{N}$-heterocycles in high yields. Although the catalyst could be easily removed by the reaction mixture using an external magnet and reused for six runs without losing catalytic activity, copper leaching into the solution of about $0.25 \%$ (after the first run) and 2.5\% (after the fifth run) was observed by AAS measurements. 


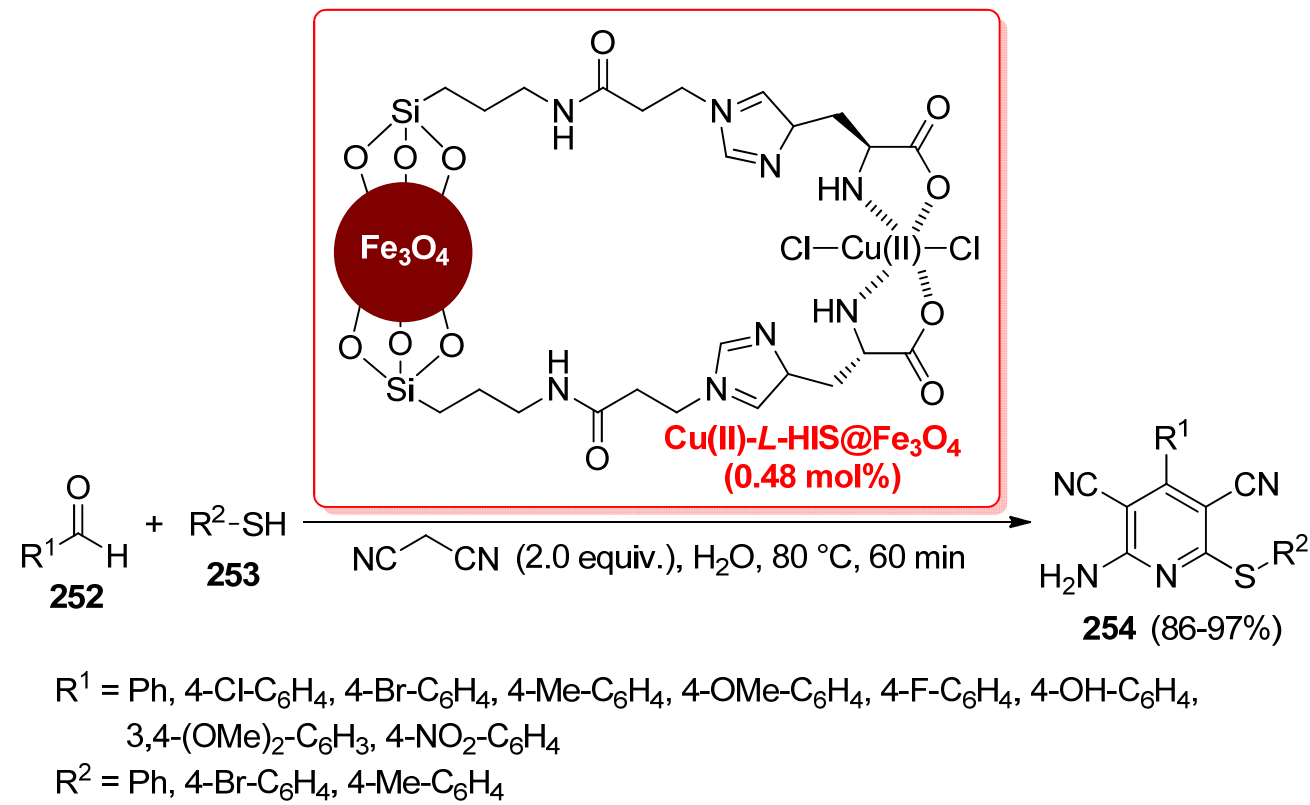

Scheme 78. Synthesis of 2-amino-3,5-dicarbonitrile-6-thio-pyridines 254 by multicomponent reaction of benzaldehydes 252, thiophenols 253 and malononitrile catalyzed by $\mathrm{Cu}(\mathrm{II})-\mathrm{L}-\mathrm{HIS} @ \mathrm{Fe}_{3} \mathrm{O}_{4}$, reported in 2016 by Guorbani-Choghamarani and co-workers.

In 2019, Gupta and co-workers functionalized magnetite $\left(\mathrm{Fe}_{3} \mathrm{O}_{4}\right)$ nanoparticles with $L$-DOPA, and the $\alpha$-amino acid portion of the resulting anchored ligand was employed for the coordination of copper(II) via treatment with $\mathrm{CuCl}_{2}$, thus generating the final supported $\mathrm{Fe}_{3} \mathrm{O}_{4}$-DOPA-Cu nanocatalyst [184]. This copper species showed remarkable activity in promoting the synthesis of 1,4-disubstituted 1,2,3-1H-triazoles 256 by tandem $\mathrm{S}_{\mathrm{N}} 2 /[3+2]$ cycloaddition of terminal alkynes 255 with benzyl bromide and $\mathrm{NaN}_{3}$, as well as the preparation of 2,4,5-trisubstituted $1 \mathrm{H}$-imidazoles 258 by the condensation-cyclization reaction of aldehydes 257 with benzyl and ammonium acetate (Scheme 79 ). The reaction conditions appear quite compatible with the canons of Green Chemistry, since they were carried out in $\mathrm{H}_{2} \mathrm{O}$ or ethanol as the solvent, under microwave irradiation for very short durations (10-18 $\mathrm{min})$.
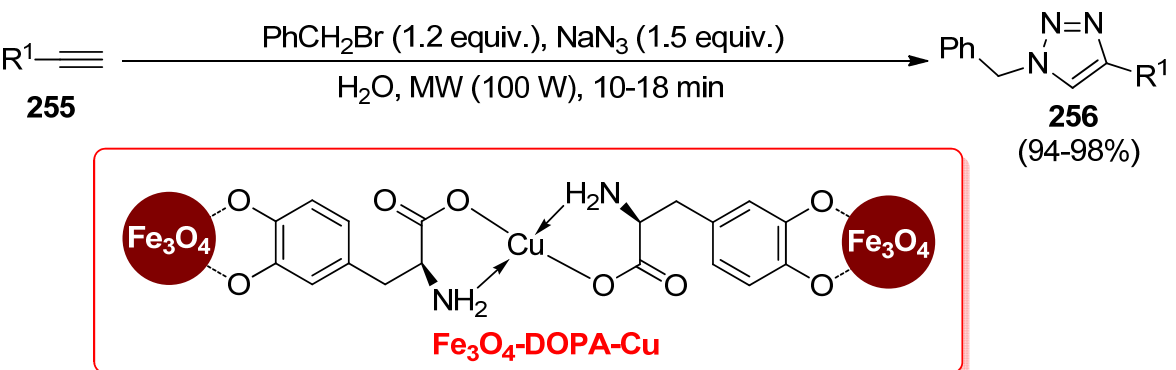

(b)

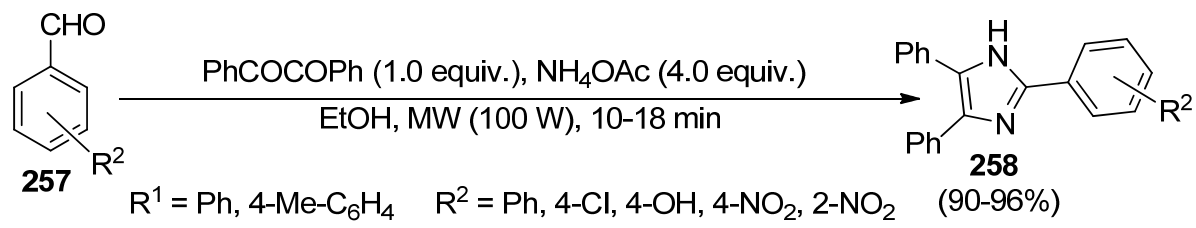

Scheme 79. Synthesis of: (a) 1,4-disubstituted 1,2,3-1H-triazoles 256 by tandem $\mathrm{S}_{\mathrm{N}} 2 /[3+2]$ cycloaddition of terminal alkynes 255 with benzyl bromide and $\mathrm{NaN}_{3}$; (b) 2,4,5-trisubstituted $1 \mathrm{H}$-imidazoles 258 by condensation-cyclization of aldehydes 257 with benzyl and $\mathrm{NH}_{4} \mathrm{OAc}$ catalyzed by $\mathrm{Fe}_{3} \mathrm{O}_{4}{ }^{-}$ DOPA-Cu, reported in 2019 by Gupta and co-workers. 
In 2017, Guorbani-Choghamarani et al. proposed the immobilization of a 2-amino-2methyl-1,3-propandiol (AMPD) ligand to $\mathrm{Fe}_{3} \mathrm{O}_{4}$ nanoparticles and the subsequent complexation of a copper(II) species to give the final $\mathrm{Fe}_{3} \mathrm{O}_{4}$-AMPD-Cu supported catalyst; it exhibited high chemical stability and catalytic activity in the synthesis of 5-substituted $1 \mathrm{H}$-tetrazoles from benzonitriles and $\mathrm{NaN}_{3}$ [185].

Very recently, the same research group recently reported the only example of a metal catalyst supported on a mineral-anchored ligand: a copper(II)-guanidine complex anchored to bohemite [186]. The mineral was treated with 3-chloropropyltrimetoxysilane, affording the corresponding chlorinated material (n-PrCl@BO-NPs); then, the coupling with guanidine ligand and the final treatment with $\mathrm{Cu}\left(\mathrm{NO}_{3}\right)_{2} \cdot 9 \mathrm{H}_{2} \mathrm{O}$ furnished the final heterogeneous catalyst $\mathrm{Cu}$-guanidine@BO-NPs. It was successfully applied to the preparation of 5-substituted $1 \mathrm{H}$-tetrazoles 260 by the reaction of benzonitriles 259 with sodium azide, carried out in PEG-400 as a sustainable solvent at $120^{\circ} \mathrm{C}$ (Scheme 80). Interestingly, the catalyst could be easily recovered by simple filtration and reused up to six times with no loss of its catalytic activity; however, no studies were carried out in order to verify the actual nature (homogeneous vs. heterogeneous) of the catalytically active species.

\subsection{Metal Catalysts Supported on Magnetic Core/Inorganic Shell Composite-Anchored Ligands}

As already described in Section 2.5, core/shell nanocomposites consisting of a magnetic core of iron oxides coated with a shell of a suitable inorganic material are emerging as new, challenging supports for metal catalysts. However, the inorganic shell of these nanocomposites - in most cases, a functionalized silica material — can then be subsequently functionalized with an organic ligand, able to complex a suitable metal species. Therefore, in this section, we take into account metal catalysts complexed to magnetic core/inorganic shell composite-anchored ligands.

Copper species are definitely the most common belonging to this class of supported catalysts, and several copper complexes bearing different organic ligands (carbene, salen, amines, triazoles and other $N$-heterocycles, dihydroxyanthraquinone, and so on) have been successfully immobilized to core/shell iron oxides@SiO $\mathrm{C}_{2}$ composites and then tested for the preparation of $N$-heterocycles.

The first system was developed in 2013 by Díez-González, Wilton-Ely et al., where they described the preparation of a $N$-heterocyclic carbene ligand, which was then connected to core/shell $\mathrm{Fe}_{3} \mathrm{O}_{4} @ \mathrm{SiO}_{2}$ nanoparticles and finally treated with $\mathrm{CuI}$ to give the final supported catalyst, named ${ }^{\mathrm{Fe}_{3}} \mathrm{O}_{4} / \mathrm{Si} \mathrm{Cu}(\mathrm{NHC})$ by the authors [153]. The present catalytic system was found to be very efficient in promoting the synthesis of 1,2,3-1H-triazoles 263 by [ $3+2$ ] cycloaddition of azides 261 with terminal alkynes 262 , carried out in water as the solvent, at room temperature or under modest thermal heating $\left(40{ }^{\circ} \mathrm{C}\right)$ for $18 \mathrm{~h}$ (Scheme 81$)$. The main advantage of $\mathrm{Fe}_{3} \mathrm{O}_{4} / \mathrm{Si} \mathrm{Cu}(\mathrm{NHC})$ is its high reusability, with up to ten consecutive runs with no significant loss of activity, together with its very easy removal by means of a hand-held magnet.

In the same year, Sardarian and co-workers reported the development of a $\mathrm{Cu}(\mathrm{II})$-salen complex immobilized on core/shell superparamagnetic $\mathrm{Fe}_{3} \mathrm{O}_{4} @ \mathrm{SiO}_{2}$ nanoparticles. First, salicylaldehyde was treated with 3-aminopropyl(triethoxy)silane to give a silylated salen ligand; then, $\mathrm{Cu}(\mathrm{OAc})_{2}$ was added to give the corresponding silylated $\mathrm{Cu}(\mathrm{II})$-salen complex, which was finally anchored to core/shell superparamagnetic $\mathrm{Fe}_{3} \mathrm{O}_{4} @ \mathrm{SiO}_{2}$ nanoparticles to give the final supported $\mathrm{Fe}_{3} \mathrm{O}_{4} @ \mathrm{SiO}_{2} /$ salen-Cu(II) catalyst [187]. Such a catalyst was successfully used for the synthesis of 1 -substituted $1 H$-tetrazoles by the reaction of anilines with sodium azide and triethyl orthoformate, as well as for the preparation of 5-substituted $1 \mathrm{H}$ - tetrazoles by the reaction of benzonitriles with sodium azide. $\mathrm{Fe}_{3} \mathrm{O}_{4} @ \mathrm{SiO}_{2} /$ salen- $\mathrm{Cu}$ (II) was used for seven consecutive runs without a significant loss of catalytic activity. This was well testified by the low amounts of $\mathrm{Cu}$ leaching into the solution, i.e., $0.2 \%$ after the first run and $5.4 \%$ after the seventh run (ICP analysis). 


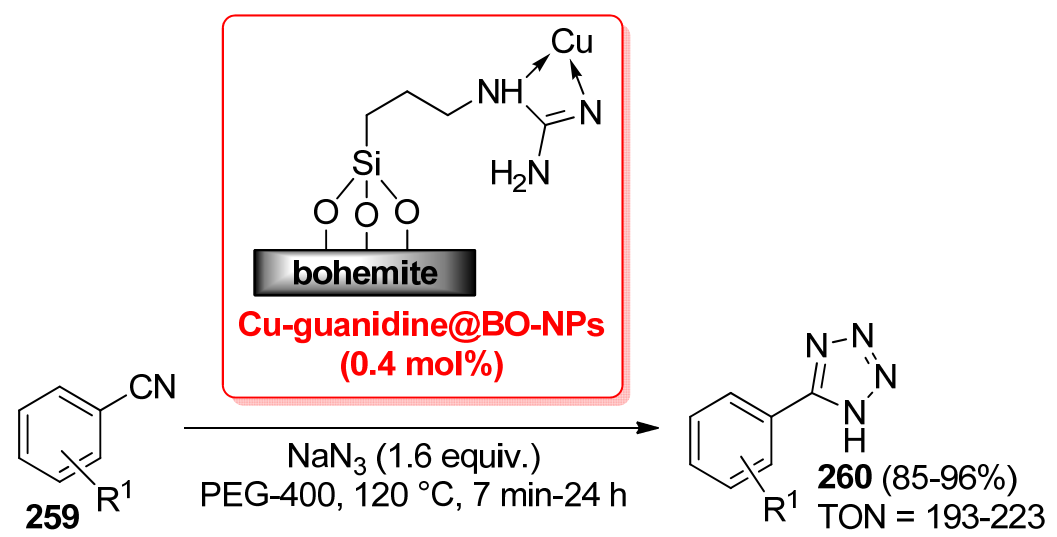

$\mathrm{R}^{1}=\mathrm{H}, 2-\mathrm{OH}, 4-\mathrm{OH}, 3-\mathrm{NO}_{2}, 4-\mathrm{NO}_{2}, 2-\mathrm{Cl}, 4-\mathrm{COMe}, 4-\mathrm{CN}, 2-\mathrm{CN}$

Scheme 80. Synthesis of 5-substituted $1 H$-tetrazoles 260 by the reaction of benzonitriles 259 with sodium azide catalyzed by Cu-guanidine@BO-NPs, reported in 2020 by Guorbani-Choghamarani et al.

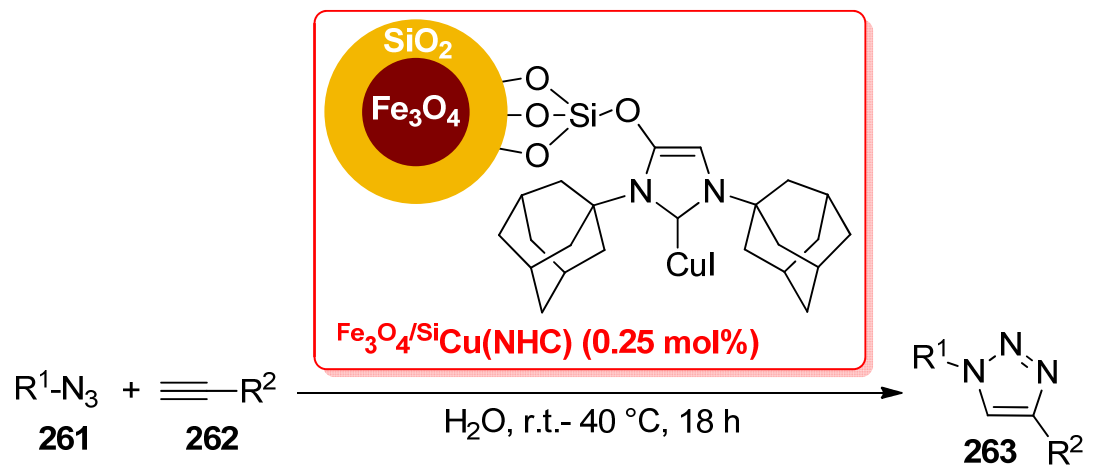

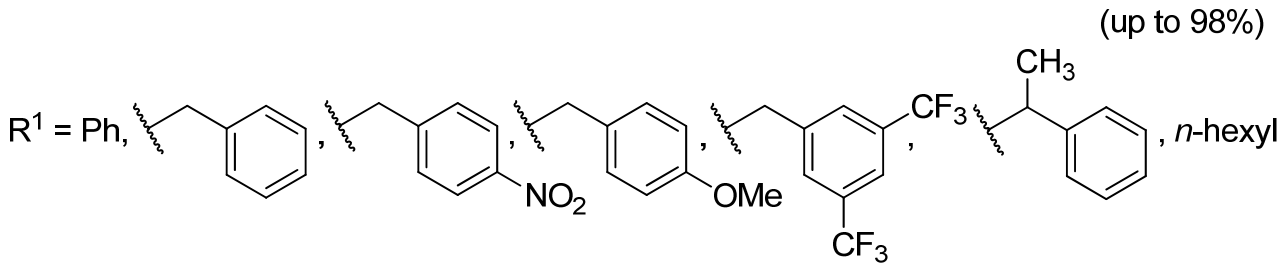

$\mathrm{R}^{2}=\mathrm{Ph}, \mathrm{SiMe}_{3}, \mathrm{C}\left(\mathrm{CH}_{3}\right)_{2} \mathrm{OH},\left(\mathrm{CH}_{2}\right)_{3} \mathrm{Cl}$, cyclopropyl, $\mathrm{CH}_{2} \mathrm{NMe}_{2}, n-\mathrm{Bu}, \mathrm{COOEt}$

Scheme 81. Synthesis of 1,2,3-1H-triazoles 263 by [3 + 2] cycloaddition of azides 261 with terminal alkynes 262 catalyzed by $\mathrm{Fe}_{3} \mathrm{O}_{4} / \mathrm{Si} \mathrm{Cu}(\mathrm{NHC})$, reported in 2013 by Díez-González, Wilton-Ely et al.

The immobilization of amine ligands to core/shell $\mathrm{Fe}_{3} \mathrm{O}_{4} @ \mathrm{SiO}_{2}$ nanoparticles was a more common approach for the development of supported copper catalysts. In 2013, Xiong et al. described a very efficient and practical approach for the synthesis of 1,2,3-1H-triazoles 266 by a tandem $\mathrm{S}_{\mathrm{N}} 2 /[3+2]$ cycloaddition of organic halides 264 and alkynes 265 [188]. The silica shell of $\mathrm{Fe}_{3} \mathrm{O}_{4} @ \mathrm{SiO}_{2}$ nanoparticles was functionalized with 3-aminopropyltrimethoxysilane (APTS) to yield an amino-functionalized composite, which was then treated with $\mathrm{CuBr}$ under DMF to give the final $\mathrm{Fe}_{3} \mathrm{O}_{4} @ \mathrm{SiO}_{2}$-APTS-CuBr supported catalyst, used at $1.46 \mathrm{~mol} \%$ of loading for the reaction of organic halides 264 and alkynes 265, carried out in $\mathrm{H}_{2} \mathrm{O} / \mathrm{PEG}$ as the solvent, under microwave irradiation $\left(80^{\circ} \mathrm{C}\right)$ for very short durations $(15-26 \mathrm{~min})$ (Scheme 82). The present protocol not only reflects many of the Twelve Principles of Green Chemistry, in terms of catalyst recyclability (Principle 1: Prevention of Waste; Principle 4: Designing Safer Chemicals; Principle 8: Catalysis), solvent sustainability (Principle 5: Safer Solvents and Auxiliaries) and low energy requirements (Principle 6: Design for Energy Efficiency), but also for its high robustness in scale-up, up to 60 times higher. 


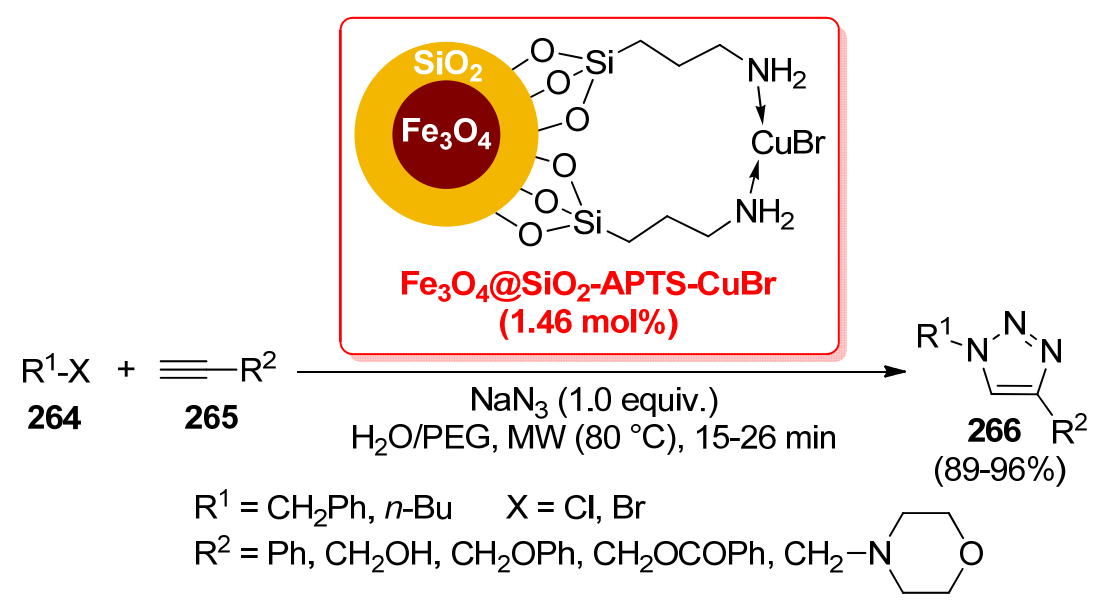

Scheme 82. Synthesis of 1,2,3-1H-triazoles 266 by a tandem $S_{N} 2 /[3+2]$ cycloaddition of organic halides 264 and alkynes 265 catalyzed by $\mathrm{Fe}_{3} \mathrm{O}_{4} @ \mathrm{SiO}_{2}-\mathrm{APTS}-\mathrm{CuBr}$, reported in 2013 by Xiong et al.

For the preparation of 1,4-disubstituted 1,2,3-1H-triazoles, good results were also described in 2016 by Paul and co-workers with the use of the magnetically recoverable copper catalyst $\mathrm{Cu}(0)-\mathrm{Fe}_{3} \mathrm{O}_{4} @ \mathrm{SiO}_{2} / \mathrm{NH}_{2}$ cel [189]. It was easily prepared via the functionalization of core/shell $\mathrm{Fe}_{3} \mathrm{O}_{4} @ \mathrm{SiO}_{2}$ nanoparticles with ethylene diamine-functionalized cellulose, followed by treatment with $\mathrm{CuCl}_{2}$ in the presence of $\mathrm{NaBH}_{4}$ as a reducing agent, which allowed the complexation of copper(0) nanoparticles to the amino groups of the hybrid support to give the final $\mathrm{Cu}(0)-\mathrm{Fe}_{3} \mathrm{O}_{4} @ \mathrm{SiO}_{2} / \mathrm{NH}_{2}$ cel catalyst. Reactions were performed at room temperature with a very low amount of the catalyst $(0.25 \mathrm{~mol} \%)$, which was sufficient to achieve the desired heterocyclic products in $90-95 \%$ yields. Firouzabadi, Sardarian et al. instead reported in 2018 the immobilization of a triazine- trimethylenediamine dendrimer on core/shell $\mathrm{Fe}_{3} \mathrm{O}_{4} @ \mathrm{SiO}_{2}$ nanoparticles and the subsequent complexation of $\mathrm{Cu}$ (II) species, to give the final supported $\mathrm{Fe}_{3} \mathrm{O}_{4} @ \mathrm{SiO}_{2}-\mathrm{TCT}-\mathrm{NH}_{2}$-dendrimer- $\mathrm{Cu}$ (II) catalyst, used at $0.4 \mathrm{~mol} \%$ loading in the synthesis of 5 -substituted $1 \mathrm{H}$-tetrazoles by a multicomponent reaction of benzaldehydes with $\mathrm{NaN}_{3}$ and hydroxylamine hydrochloride [190]. These transformations, occurring through a tandem oxime formation/[3 + 2] cycloaddition, were carried out in $\mathrm{H}_{2} \mathrm{O}$ as the solvent, at $70{ }^{\circ} \mathrm{C}$ for $30-120 \mathrm{~min}$, and allowed for good reusability of the catalyst (up to seven consecutive runs with no significant decrease in the products yields): features of pivotal importance in the context of Green Chemistry.

Triazoles are definitely among the most common ligands for copper species; therefore, it should not be surprising that these systems have been successfully immobilized to core/shell iron oxides@SiO $\mathrm{S}_{2}$ nanoparticles for the development of supported Cu catalysts. In most cases, these materials have been applied as recoverable and reusable catalytic systems for the synthesis of 1,4-disubstituted 1,2,3-1H-triazoles by Huisgen $[3+2]$ cycloaddition reactions of organic azides with terminal alkynes. In 2014, Astruc and co-workers reported the preparation of a tris(triazolyl)-CuBr complex anchored to core/shell maghemite/silica nanoparticles. First of all, $\gamma-\mathrm{Fe}_{2} \mathrm{O}_{3} @ \mathrm{SiO}_{2}$ was treated with (3-chloropropyl)trimethoxysilane in order to chlorinate the inorganic shell of the nanocomposite; then, the tris(1-benzyl-1H-1,2,3-triazol-4-yl) methanol (TBTM) ligand was added in the presence of $\mathrm{NaH}$ as the base, affording the TBTM- anchored system; finally, treatment with $\mathrm{CuBr}$ afforded the final supported $\gamma-\mathrm{Fe}_{2} \mathrm{O}_{3} @ \mathrm{SiO}_{2}-\mathrm{TBTM}-\mathrm{CuBr}$ (Scheme 83a) [191]. In 2015, Moghaddam et al. instead described a different copper catalyst immobilized onto a triazole-functionalized core/shell $\mathrm{Fe}_{3} \mathrm{O}_{4} @ \mathrm{SiO}_{2}$, named [MNPs@FGly] $[\mathrm{Cl}] . \mathrm{Fe}_{3} \mathrm{O}_{4} @ \mathrm{SiO}_{2}$ nanoparticles were functionalized with 3- glycidoxypropyltrimethoxysilane, to give the corresponding epoxide-functionalized composite; then, tandem $\mathrm{S}_{N} 2 /[3+2]$ cycloaddition with sodium azide and phenylacetylene afforded the anchored ligand, which was finally charged with $\mathrm{CuCl}_{2}$ in the presence of sodium ascorbate as the reducing agent to produce the final [MNPs@FGly][Cl] catalyst (Scheme 83b) [192]. In 2016, the same group proposed the use of 
$\alpha$-amidotriazole-functionalized core/shell $\mathrm{Fe}_{3} \mathrm{O}_{4} @ \mathrm{SiO}_{2}$ as a support for the complexation of copper species. First, (3-aminopropyl)triethoxysilane was used for the functionalization of the inorganic shell of magnetic nanoparticles with $\mathrm{NH}_{2}$ groups; then, an ester-amide exchange reaction was performed by treatment with ethyl 2-(4-phenyl-1 $\mathrm{H}-1,2,3-$ triazol-1$\mathrm{yl}$ )acetate to give the anchored triazole ligand; finally, the complexation of $\mathrm{CuCl}_{2}$ afforded the final material [MNPs@APTA] $\left[\mathrm{Cl}_{2}\right.$ ] (Scheme 83c) [193]. These three copper catalysts exhibited high efficiency in the synthesis of 1,4-disubstituted 1,2,3-1H-triazoles, carried out in aqueous reaction media under mild conditions; in particular, if Astruc's $\gamma-\mathrm{Fe}_{2} \mathrm{O}_{3} @ \mathrm{SiO}_{2}-$ TBTM-CuBr showed modest recyclability, with a decrease in the product yields from the second run, Moghaddam's [MNPs@FGly][Cl] and [MNPs@FGly][Cl] showed almost the same catalytic activity after ten consecutive runs, with no significant metal leaching into the solution.

Very recently, Habibi and co-workers instead reported the preparation of a copper(II) species coordinated to an amino-triazole (AT) ligand immobilized to $\mathrm{Fe}_{3} \mathrm{O}_{4} @ \mathrm{SiO}_{2}$ nanoparticles, named $\mathrm{Fe}_{3} \mathrm{O}_{4} @ \mathrm{SiO}_{2}$-CPTMS-AT- $\mathrm{Cu}$. Such a catalyst resulted in being very efficient in promoting the preparation of 1 -substituted $1 H$-tetrazoles 268 by the reaction of $\alpha$-amino acids 267 with $\mathrm{NaN}_{3}$ (1.0 equiv.) and triethylorthoformate (3.0 equiv.), performed in ethanol at $80^{\circ} \mathrm{C}$ for $1-7 \mathrm{~h}$ (Scheme 84) [194]. Unfortunately, no studies of heterogeneity (hot filtration tests) were carried out, thus it was not possible to understand its real impact in the field of Green Chemistry.

Other $\mathrm{N}$-heterocycles are less common than triazoles as ligands for $\mathrm{Cu}$ species; however, in some cases, they have been covalently anchored to core/shell $\mathrm{Fe}_{3} \mathrm{O}_{4} @ \mathrm{SiO}_{2}$ nanoparticles for the development of supported Cu catalysts. In 2018, Pore and co-workers reported the preparation of a copper(II)/acetyl pyridine complex (Cu-ACP) immobilized through an iminopropyl moiety to silica-coated magnetite catalyst, named $\mathrm{Cu}-\mathrm{ACP}-\mathrm{Am}-$ $\mathrm{Fe}_{3} \mathrm{O}_{4} @ \mathrm{SiO}_{2}$ [195]. Further, in this case, the supported material was applied as a catalyst in the synthesis of 1,4- disubstituted 1,2,3-1H-triazoles 271 by a three-component tandem $\mathrm{S}_{\mathrm{N}} 2 /[3+2]$ cycloaddition reaction of benzyl bromides 269 (1.0 equiv.) with $\mathrm{NaN}_{3}$ (1.1 equiv.) and terminal alkynes 270 (1.0 equiv.), carried out in only $15-20 \mathrm{~min}$ at $80^{\circ} \mathrm{C}$ using ethanol as the solvent (Scheme 85). The protocol appeared quite appealing in the frame of Green Chemistry, thanks to the following features: the choice of a tandem process, limiting the number of isolation/purification steps, the use of a sustainable reaction medium and the modest energy requirements due to the very short reaction times. Furthermore, moderate recyclability of the supported $\mathrm{Cu}-\mathrm{ACP}-\mathrm{Am}-\mathrm{Fe}_{3} \mathrm{O}_{4} @ \mathrm{SiO}_{2}$ catalyst was observed up to six consecutive runs with only a modest reduction in the product yields.

In 2014, Tajbakhsh et al. reported an appealing strategy for the preparation of a magnetically recoverable MNP@BiimCu(I) catalyst based on a biimidazole $\mathrm{Cu}(\mathrm{I})$ complex, involving the covalent grafting of biimidazole on chloride-functionalized core/shell $\mathrm{Fe}_{3} \mathrm{O}_{4} @ \mathrm{SiO}_{2}$ nanoparticles, followed by the complexation of $\mathrm{CuI}$ [196]. Such a catalyst was successfully evaluated (1.2 mol\%) in the synthesis of imidazo[1,2-a]pyridines 275 by a three-component coupling (TCC) reaction of 2-aminopyridines 272 with aromatic or aliphatic aldehydes 273 (1.0 equiv.) and alkynes 274 (1.1 equiv.), performed in aqueous media under reflux for 5-8 h (Scheme 86). At the end of these reactions, MNP@BiimCu(I) was easily removed using an external magnet, and after washing and vacuum drying, it was reused for ten consecutive runs without a significant decrease in its catalytic activity, thus demonstrating its exceptionally high thermal and chemical stability. In a following study, the same research group applied the same magnetically recoverable $\mathrm{MNP} @ \mathrm{BiimCu}(\mathrm{I})$ to the synthesis in good to excellent yields of 1,4-disubstituted 1,2,3-1H-triazoles by means of Huisgen 1,3-dipolar cycloaddition of primary halides with alkynes and $\mathrm{NaN}_{3}$ [197]. Moreover, in this case, the catalyst was easily recovered using an external magnet and then reused for consecutive cycles with no loss of activity. 
(a)

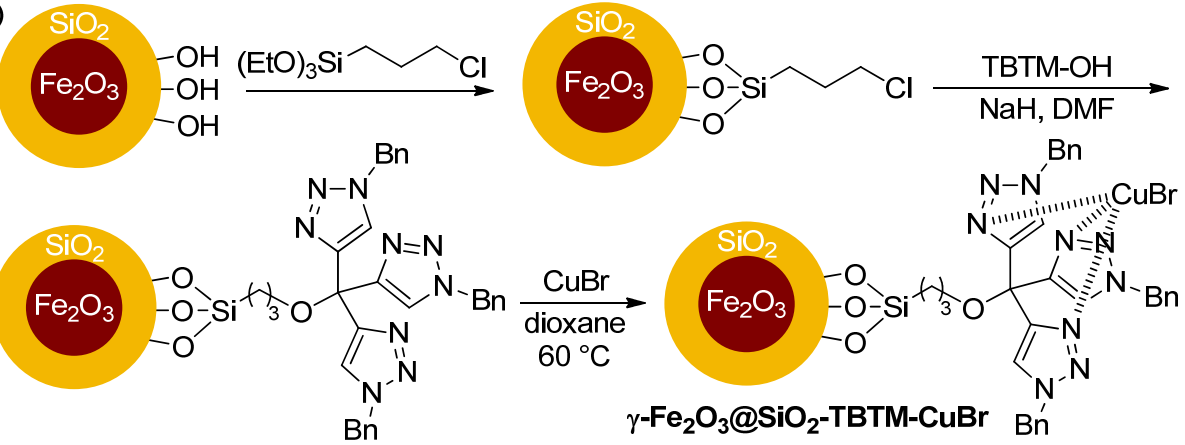

(b)

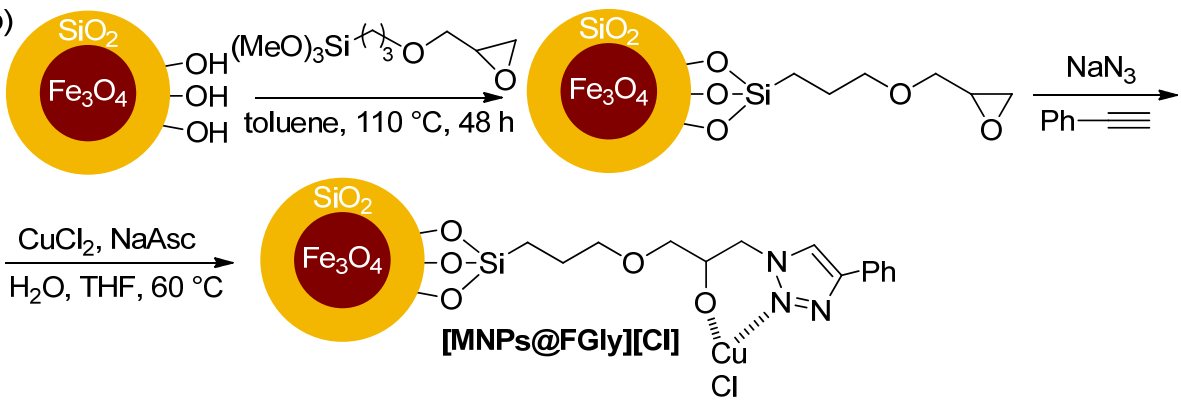

(c)

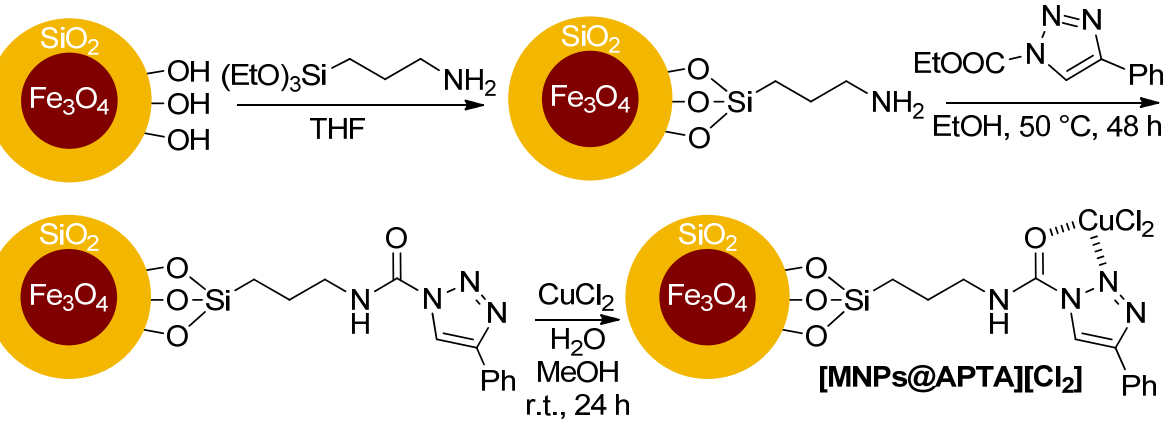

Scheme 83. Schematic route for the immobilization of copper-triazole complexes on magnetic iron oxides core/silica shell composite applied for the synthesis of 1,4-disubstituted 1,2,3-1H-triazoles by [3 + 2] cycloaddition: (a) $\gamma-\mathrm{Fe}_{2} \mathrm{O}_{3} @ \mathrm{SiO}_{2}-\mathrm{TBTM}-\mathrm{CuBr}$; (b) [MNPs@FGly][Cl]; (c) [MNPs@APTA] $\left[\mathrm{Cl}_{2}\right]$.

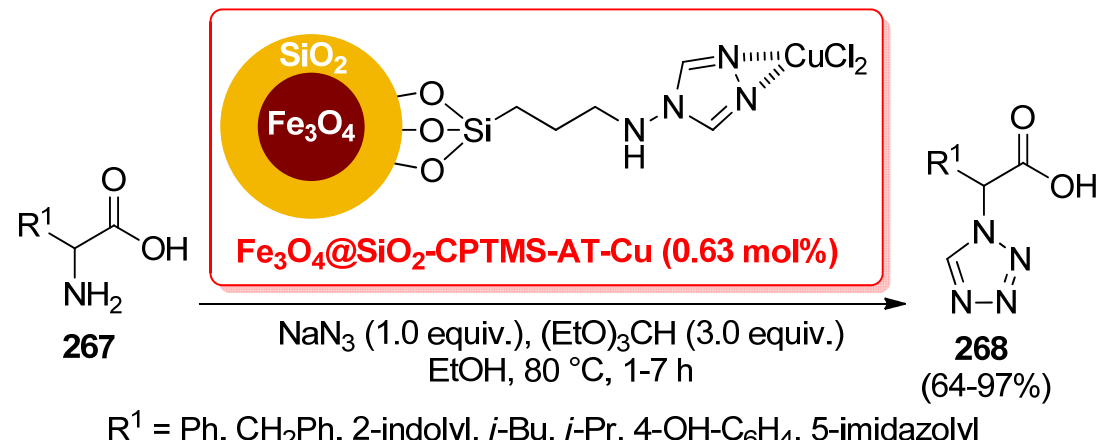

Scheme 84. Synthesis of 1 -substituted $1 H$-tetrazoles 268 by reaction of $\alpha$-amino acids 267 with $\mathrm{NaN}_{3}$ and triethylorthoformate catalyzed by $\mathrm{Fe}_{3} \mathrm{O}_{4} @ \mathrm{SiO}_{2}-\mathrm{CPTMS}-\mathrm{AT}-\mathrm{Cu}$, reported in 2019 by Habibi et al. 


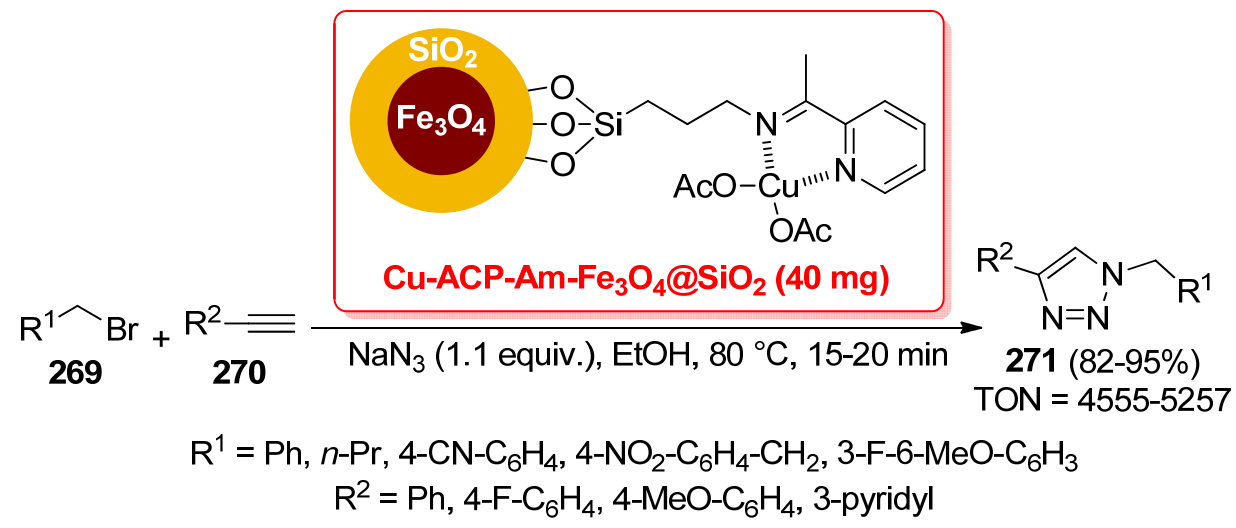

Scheme 85. Synthesis of triazoles 271 by tandem $\mathrm{S}_{\mathrm{N}} 2 /[3+2]$ cycloaddition reaction of benzyl bromides 269 with $\mathrm{NaN}_{3}$ and terminal alkynes 270 catalyzed by $\mathrm{Cu}-\mathrm{ACP}-\mathrm{Am}-\mathrm{Fe}_{3} \mathrm{O}_{4} @ \mathrm{SiO}_{2}$, reported in 2018 by Pore and co-workers.

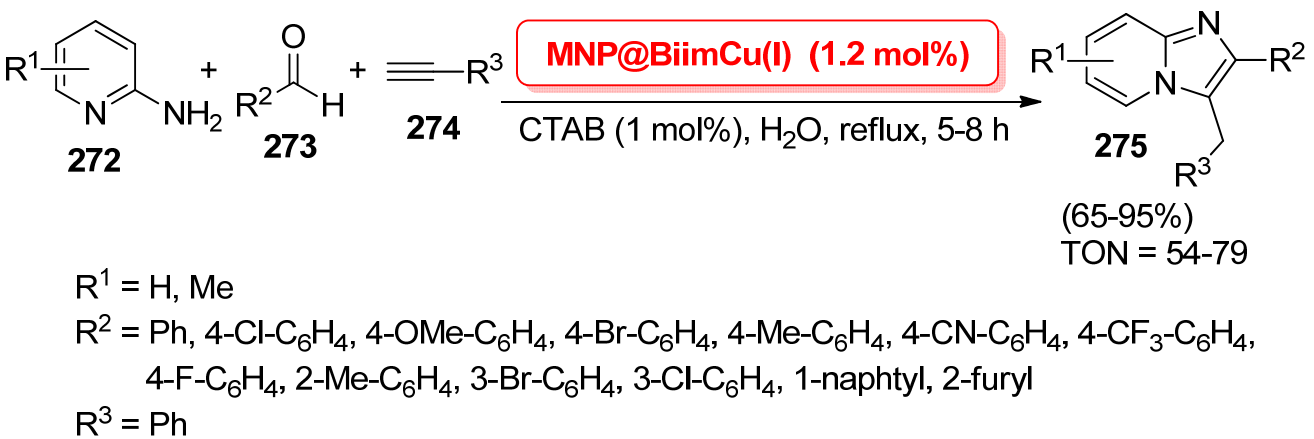

Scheme 86. Synthesis of imidazo[1,2-a]pyridines 275 by three-component coupling (TCC) reaction of 2-aminopyridines 272 with aldehydes 273 and alkynes 274 catalyzed by MNP@BiimCu(I), reported in 2014 by Tajbakhsh et al.

Esmaeilpour and co-workers studied, in detail, the development of oxygen ligandbased copper complexes immobilized on core/shell $\mathrm{Fe}_{3} \mathrm{O}_{4} @ \mathrm{SiO}_{2}$ nanoparticles as magnetically recoverable catalysts for several chemical reactions, including the synthesis of $N$-heterocycles. In 2016, they reported the preparation of a 1,4-dihydroxyanthraquinonecopper(II) complex immobilized on superparamagnetic $\mathrm{Fe}_{3} \mathrm{O}_{4} @ \mathrm{SiO}_{2}$ nanoparticles as an efficient and environmentally friendly catalytic system, named $\mathrm{Fe}_{3} \mathrm{O}_{4} @ \mathrm{SiO}_{2}-\mathrm{DAQ}-\mathrm{Cu}$ (II), used for the synthesis of (i) 1-substituted $1 \mathrm{H}$-tetrazoles 277 by the reaction of anilines 276 with sodium azide and triethyl ortho-formate, carried out under solvent-free conditions, at $100{ }^{\circ} \mathrm{C}$ for $0.75-3 \mathrm{~h}$ (Scheme 87a) [198]; (ii) 5-substituted $1 \mathrm{H}$-tetrazoles 279 by the reaction of benzonitriles 278 with sodium azide, performed in DMF at $100{ }^{\circ} \mathrm{C}$ for $2.5-6 \mathrm{~h}$ (Scheme 87b) [198]; (iii) 1-aryl-1,2,3-1H-triazoles 282 by the reaction of aryl boronic acids 280 with sodium azide and terminal alkynes 281, carried out in $\mathrm{H}_{2} \mathrm{O} / \mathrm{CH}_{3} \mathrm{CN}(1: 1 \mathrm{v} / \mathrm{v})$ as the reaction medium, at room temperature for 2-6 h (Scheme 87c) [199]. In all these protocols, the copper loading was quite low (ranging between 0.5 and $0.9 \mathrm{~mol} \%$ ), and the $\mathrm{Fe}_{3} \mathrm{O}_{4} @ \mathrm{SiO}_{2}-\mathrm{DAQ}-\mathrm{Cu}(\mathrm{II})$ catalyst was quite efficiently recycled for six consecutive runs. Moreover, TEM analysis carried out after the sixth cycle did not observe any significant change in the morphology of the catalyst, although some nanoparticles might have aggregated onto the surfaces of the core/shell matrix. Despite the fact this catalyst was compatible with some appealing features in terms of sustainability (the use of solvent-free conditions in the synthesis of 1-substituted $1 H$-tetrazoles 277 and an aqueous medium in the synthesis of triazoles $\mathbf{2 8 2}$, as well as the need for short reaction times), some issues still remained, in particular the choice of DMF as the solvent for the preparation of 5-substituted 
$1 H$-tetrazoles 279 and the need for high temperatures for the synthesis of both 277 and 279, thus limiting the interest of $\mathrm{Fe}_{3} \mathrm{O}_{4} @ \mathrm{SiO}_{2}-\mathrm{DAQ}-\mathrm{Cu}(\mathrm{II})$ in the frame of Green Chemistry.

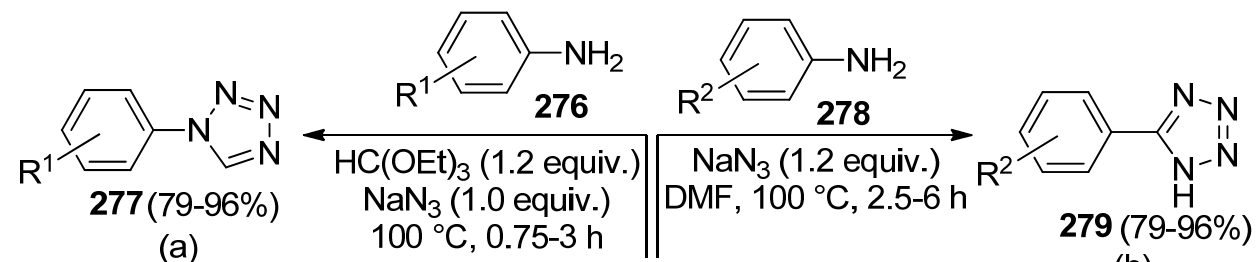

(a)

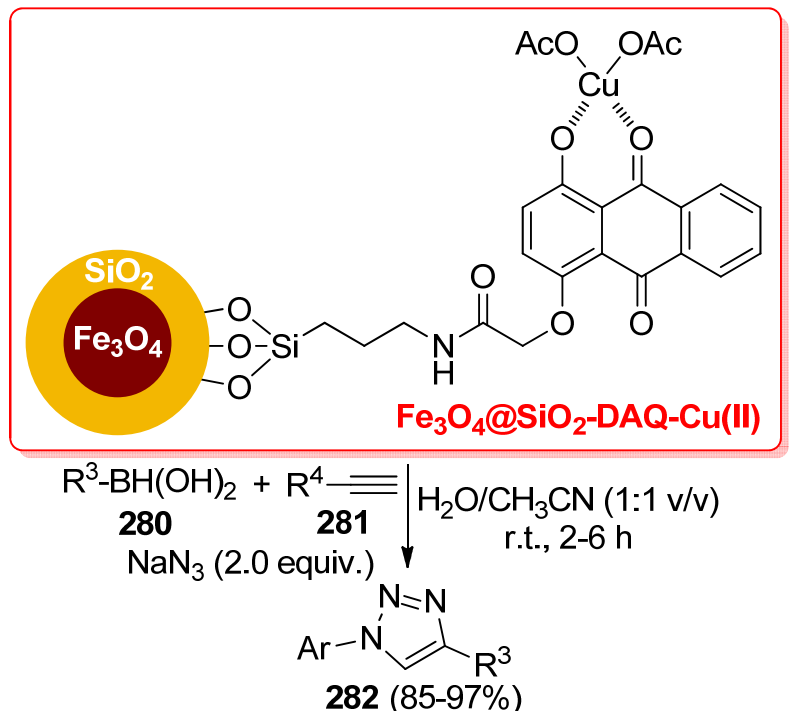

(b)

$$
\begin{gathered}
\mathrm{R}^{1}=\mathrm{H}, 4-\mathrm{Me}, 4-\mathrm{OMe}, 4-\mathrm{Br}, 4-\mathrm{Cl}, 4-\mathrm{NO}_{2}, 4-\mathrm{COMe} \\
\mathrm{R}^{2}=\mathrm{H}, 4-\mathrm{Me}, 4-\mathrm{OMe}, 4-\mathrm{OH}, 4-\mathrm{Br}, 4-\mathrm{Cl}, 4-\mathrm{NO}_{2}, 2-\mathrm{CN}, 4-\mathrm{CN} \\
\mathrm{R}^{3}=\mathrm{Ph}, \mathrm{cyclohexyl} \text {, 1-octyl } \\
\mathrm{R}^{4}=\mathrm{Ph}, \text { 4- } \mathrm{PhO}_{-} \mathrm{C}_{6} \mathrm{H}_{4}, 3-\mathrm{CHO}-\mathrm{C}_{6} \mathrm{H}_{4}, 4-\mathrm{OH}-\mathrm{C}_{6} \mathrm{H}_{4}, 4-\mathrm{MeO}_{6}-\mathrm{C}_{6} \mathrm{H}_{4} \text {, 3-thiophen, 2-naphthyl }
\end{gathered}
$$

Scheme 87. Synthesis of (a) 1-substituted $1 H$-tetrazoles 277; (b) 5-substituted $1 H$-tetrazoles 279; (c) 1-aryl-1,2,3-1H-triazoles 282 catalyzed by $\mathrm{Fe}_{3} \mathrm{O}_{4} @ \mathrm{SiO}_{2}-\mathrm{DAQ}-\mathrm{Cu}(\mathrm{II})$, reported in 2016 by Esmaeilpour and co-workers.

In 2018, the same research group instead proposed the green synthesis of 5-substituted $1 \mathrm{H}$-tetrazoles in excellent yields through the click reaction of aliphatic and aromatic aldehydes with hydroxylamine hydrochloride and sodium azide, performed for $4 \mathrm{~h}$ in refluxing $\mathrm{H}_{2} \mathrm{O}$ in the presence of copper(II) supported on polyvinyl alcohol immobilized on core/shell $\mathrm{Fe}_{3} \mathrm{O}_{4} @ \mathrm{SiO}_{2}$ nanoparticles (named $\mathrm{Fe}_{3} \mathrm{O}_{4} @ \mathrm{SiO}_{2}-\mathrm{PVA}-\mathrm{Cu}^{2+}$ in the manuscript) [200]. In particular, the catalyst showed very good recyclability in these reactions, up to seven times, without any change in catalytic activity, as well as in the morphology of copper nanoparticles verified by SEM analysis. However, the amount of copper leaching into the solution increased from $0.53 \%$ in the first run to 4.41 in the seventh run, thus demonstrating the possibility of metal contamination that could limit the overall sustainability of the synthetic protocol in the frame of Green Chemistry.

Although the inorganic shell of all the above-described nanocomposites was based on functionalized silica materials, other inorganic matrices have also been used in a few cases. In this context, in 2016, Akhlaghinia et al. described the immobilization of copper(II) species on guanidinated epibromohydrin functionalized core/shell $\gamma-\mathrm{Fe}_{2} \mathrm{O}_{3} @ \mathrm{TiO}_{2}$ nanoparticles (named $\gamma-\mathrm{Fe}_{2} \mathrm{O}_{3} @ \mathrm{TiO}_{2}-\mathrm{EG}-\mathrm{Cu}^{\mathrm{II}}$ ) [201]. In particular, after the preparation of titania-coated maghemite nanocomposites, treatment with epibromohydrin (used as both the reagent and solvent) at $60^{\circ} \mathrm{C}$ for $24 \mathrm{~h}$ and subsequent treatment with guanidine hydrochloride and $\mathrm{NaHCO}_{3}$ in toluene afforded the anchored ligand, which was finally used for the 
immobilization of copper(II) species by treatment with $\mathrm{Cu}(\mathrm{OAc})_{2} \cdot \mathrm{H}_{2} \mathrm{O}$ in EtOH at room temperature for $4 \mathrm{~h}$, to give the supported $\gamma-\mathrm{Fe}_{2} \mathrm{O}_{3} @ \mathrm{TiO}_{2}-\mathrm{EG}-\mathrm{Cu}^{\mathrm{II}}$ material. This system was then used as a catalyst for the preparation of 1,4-disubstituted 1,2,3-1H-triazoles 285 by three-component, one-pot azide formation/[3 +2$]$ cycloaddition reactions of bromides 283, terminal alkynes 284 and sodium azide, performed in water at $50{ }^{\circ} \mathrm{C}$ for $5-75 \mathrm{~min}$ (Scheme 88). It is worth emphasizing that the authors proved this system to be a heterogeneous catalyst, and it was easily isolated and reused for six consecutive runs without a loss of activity and negligible metal leaching after the sixth run, as verified by ICP analysis. The heterogeneity of $\gamma-\mathrm{Fe}_{2} \mathrm{O}_{3} @ \mathrm{TiO}_{2}-\mathrm{EG}-\mathrm{Cu}^{\mathrm{II}}$, in combination with the use of $\mathrm{H}_{2} \mathrm{O}$ as an environmentally friendly solvent and with the limited energy requirements due to the rather mild experimental conditions, makes the present protocol of high interest from the point of view of Green Chemistry.

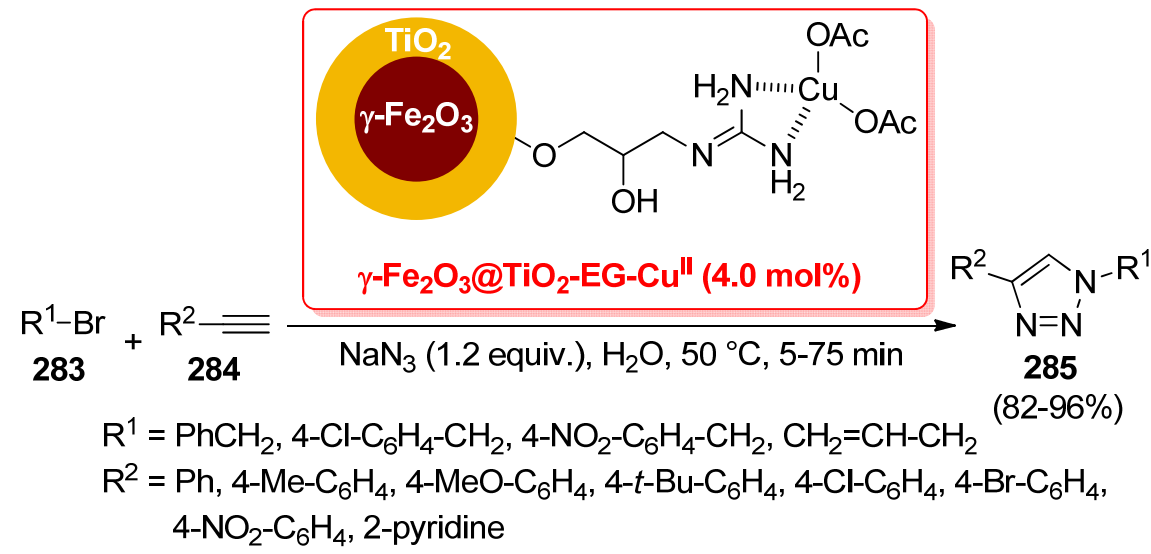

Scheme 88. Synthesis of triazoles 285 by tandem azide formation/[ $3+2$ ] cycloaddition reaction of organic bromides 283 with $\mathrm{NaN}_{3}$ and terminal alkynes 284 catalyzed by $\gamma-\mathrm{Fe}_{2} \mathrm{O}_{3} @ \mathrm{TiO}_{2}-\mathrm{EG}-\mathrm{Cu}^{\mathrm{II}}$, reported in 2016 by Akhlaghinia et al.

For the sake of completeness, we also briefly describe the few examples of applications of metal species coordinated to magnetic core/inorganic shell composite-anchored ligands to the synthesis of $N$-heterocycles. In 2015, Lee, Zhong and co-workers described the immobilization of a Grubbs-Hoveyda-type ruthenium-carbene complex on silica-coated magnetic nanoparticles through an imidazolium salt linker, tested in the ring-closing metathesis of dienes to give several cyclic compounds, including dihydropyrroles, tetrahydropyridines and tetrahydroazepines, performed in $\mathrm{CH}_{2} \mathrm{Cl}_{2}$ at room temperature with low Ru loading (0.85 mol\%) [202].

Salehzadeh et al. reported in 2016 a dioxomolybdenum complex covalently immobilized on core/shell magnetic $\mathrm{Fe}_{3} \mathrm{O}_{4} @ \mathrm{SiO}_{2}$ (named $\mathrm{Fe}_{3} \mathrm{O}_{4} @ \mathrm{Si} @ \mathrm{MoO}_{2}$ ), which was successfully tested in the synthesis of pyrazoles by three-component condensation of aromatic aldehydes with malononitrile and phenylhydrizine, performed at room temperature under solvent-free conditions [203]. Interestingly, the $\mathrm{Fe}_{3} \mathrm{O}_{4} @ \mathrm{Si} @ \mathrm{MoO}_{2}$ catalyst could be recovered in a facile manner from the reaction mixture and recycled for eight runs without a loss of activity. Moreover, the determination of molybdenum in the pyrazole products after the catalyst removal by ICP-AES analysis showed no metal leaching occurring during the process. Zhang et al. proposed the preparation of the $\mathrm{CoFe}_{2} \mathrm{O}_{4} @ \mathrm{SiO}_{2}-\mathrm{PrNH}_{2}-\mathrm{Mo}(\text { acac })_{2}$ catalyst, that is, a molybdenyl acetylacetonate complex covalently anchored, through a 3-aminopropyltriethoxysilane spacer, to silica-coated cobalt ferrite nanoparticles [204]. Such a supported system showed remarkable activity and reusability (five consecutive cycles) in the preparation of various pyrroles in good to excellent yields by a one-pot, fourcomponent reaction of amines, aldehydes, nitromethane and 1,3-dicarbonyl compounds, carried out at $90^{\circ} \mathrm{C}$ under solvent-free conditions. In both protocols, the use of an efficient and recoverable catalyst, working through a merely heterogeneous mechanism, in combi- 
nation with the use of solvent-free conditions, allowed them to respect most of the Twelve Principles of Green Chemistry: Principle 1: Prevention of Waste; Principle 4: Designing Safer Chemicals; Principle 6: Design for Energy Efficiency; Principle 8: Catalysis.

In 2014, Zhang et al. also reported an interesting antimony(III) supported catalyst, named $\mathrm{CoFe}_{2} \mathrm{O}_{4} @ \mathrm{SiO}_{2}-\mathrm{DABCO}-\mathrm{Sb}$, obtained in three steps: (i) treatment of silica-coated cobalt ferrite nanoparticles with 3-chloropropyltrimethoxysilane, to give the corresponding chlorinated material; (ii) a reaction with 1,4-diazabicyclo[2.2.2]octane (DABCO) in refluxing acetone, affording a protonated $\mathrm{DABCO}$ ligand covalently anchored to magnetic nanoparticles; and (iii) a final reaction of $\mathrm{CoFe}_{2} \mathrm{O}_{4} @ \mathrm{SiO}_{2}-\mathrm{DABCO}$ with antimony trichloride in refluxing acetone, affording $\mathrm{CoFe}_{2} \mathrm{O}_{4} @ \mathrm{SiO}_{2}-\mathrm{DABCO}-\mathrm{Sb}$ [205]. Therefore, in this case, antimony(III) was actually connected to the protonated DABCO ligand by an electrostatic interaction with $\mathrm{SbCl}_{4}{ }^{-}$. Such a catalyst was then evaluated in the one-pot, three-component synthesis of multi-substituted pyrroles $\mathbf{2 8 9}$ in the reaction of nitroolefins 286, primary amines 287 and 1,3 diketones 288 (Scheme 89). The $\mathrm{CoFe}_{2} \mathrm{O}_{4} @ \mathrm{SiO}_{2}-\mathrm{DABCO}$ $\mathrm{Sb}$ supported catalyst could be easily recovered using an external magnet and reused up to five times with no loss of catalytic activity. A further $\mathrm{Sb}$ (III) supported catalyst has been developed by the same authors by exploiting a similar electrostatic interaction of $\mathrm{SbCl}_{4}{ }^{-}$ with a cationic $N$-methylimidazole unit anchored to silica-coated maghemite nanoparticles, indicated as $\gamma-\mathrm{Fe}_{2} \mathrm{O}_{3} @ \mathrm{SiO}_{2}-\mathrm{Sb}-\mathrm{IL}$ [206]. Such a catalytic system, which is, in other words, a supported antimony(III)-based ionic liquid, has been successfully evaluated as a recoverable catalyst for the high-yield synthesis of $N$-substituted pyrroles by thee Clauson-Kaas reaction in an aqueous medium. Hot filtration tests revealed no further product formation after catalyst removal, thus confirming the absence of antimony(III) species leached into the solution.

\subsection{Metal Catalysts Supported on Magnetic Core/Organic Shell Composites}

In the previous sections, Sections 2.5 and 4.4, we described a large number of metal catalysts immobilized (directly or through an intermediate organic ligand) on magnetic core/inorganic shell nanocomposites. A less investigated approach is the use, as a support for metal species, of core/shell composites based on a magnetic core of iron oxides coated with a shell of a suitable organic material. The few examples of such systems, which have been used for the preparation of $N$-heterocycles, are listed below.

Oligo/polysaccharide-based materials represent one of the most typical organic shells used for this class of composites. In 2016, Jafarpour and Rezaeifard described the synthesis of a zirconium-Schiff base complex anchored on starch-coated $\gamma$ - $\mathrm{Fe}_{2} \mathrm{O}_{3}$ nanoparticles (named $\mathrm{ZrOL}_{2} @ S M N P$ by the authors), showing remarkable activity in the synthesis of quinoxalines and pyridopyrazines 292 by the condensation of 1,2-diamines 290 with 1,2-diketones 291 [207]. In particular, reactions were carried out in ethanol as a solvent, at $60{ }^{\circ} \mathrm{C}$ for $2-100 \mathrm{~min}$, with a very low quantity of the $\mathrm{ZrOL}_{2} @ S M N P$ catalyst $(0.004 \mathrm{~mol} \%)$, which could be recycled at least four times without a loss of activity (Scheme 90).

Maleki et al. instead proposed the development of silver nanoparticles supported on cellulose-coated maghemite composites (indicated as cellulose $/ \gamma-\mathrm{Fe}_{2} \mathrm{O}_{3} / \mathrm{Ag}$ ). Such a catalyst was successfully used in the synthesis of 2,4,5-trisubstituted- $1 H$-imidazoles by one-pot, three-component condensation of benzaldehydes with benzyl and ammonium acetate, performed at $100^{\circ} \mathrm{C}$ under solvent-free conditions for $10 \mathrm{~min}$ [208]. The catalyst was recycled for five consecutive runs without a loss of activity, but neither studies of silver leaching nor hot filtration tests have been carried out. Therefore, although the authors defined this catalyst as heterogeneous, we cannot actually exclude the occurrence of metal leaching into the solution. 


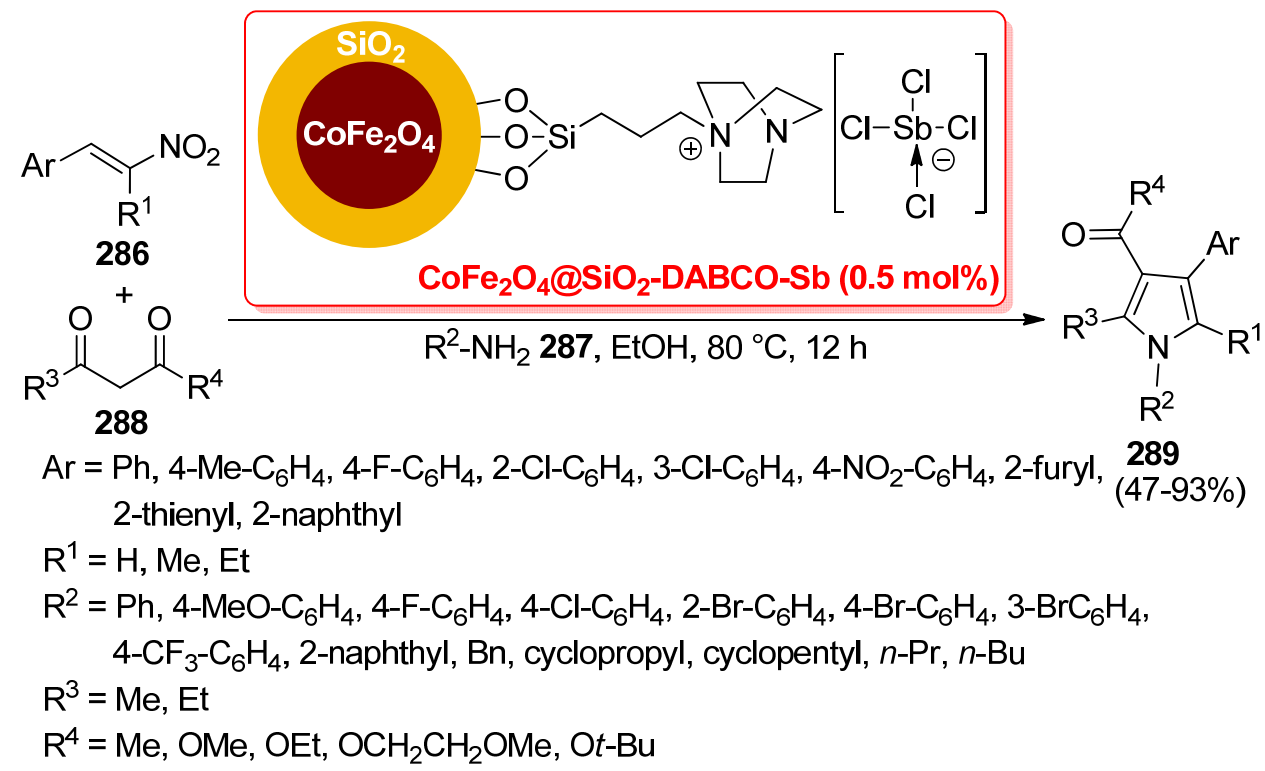

Scheme 89. Synthesis of pyrroles 289 by one-pot three-component reaction of nitroolefins 286, primary amines 287 and 1,3 diketones 288 catalyzed by $\mathrm{CoFe}_{2} \mathrm{O}_{4} @ \mathrm{SiO}_{2}-\mathrm{DABCO}-\mathrm{Sb}$, reported in 2014 by Zhang et al.

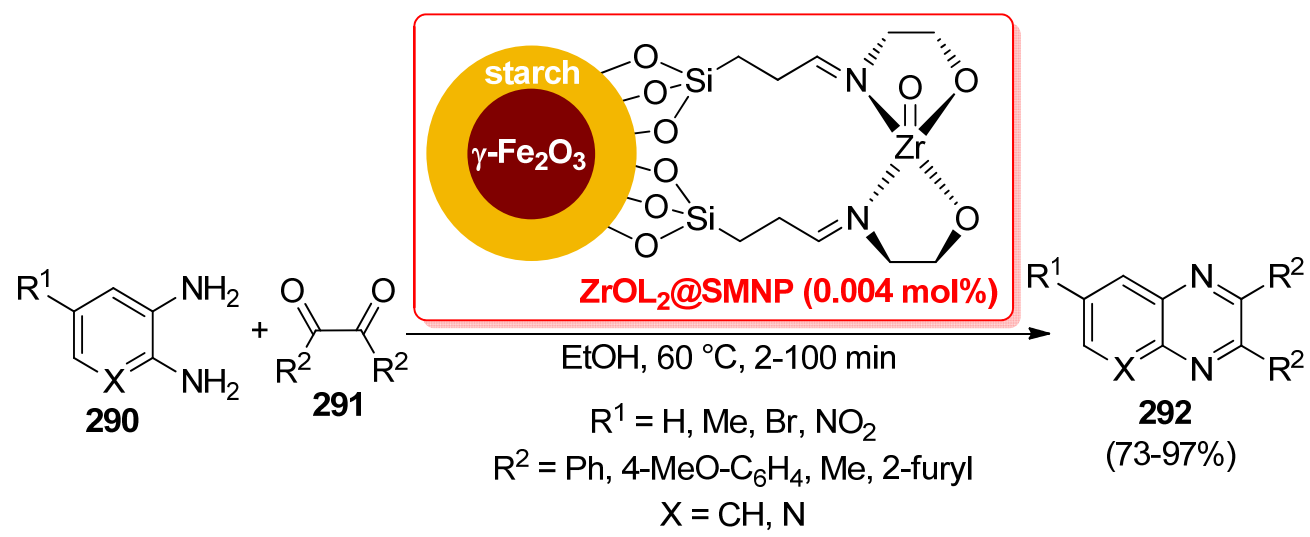

Scheme 90. Synthesis of quinoxalines and pyridopyrazines 292 by the condensation of 1,2-diamines 290 with 1,2-diketones 291 catalyzed by ZrOL $2 @ S M N P$, reported in 2016 by Jafarpour and Rezaeifard.

In 2016, Shafiee and co-workers reported the preparation of $\mathrm{Cu} \beta \beta-\mathrm{CD} @ S P I O N s$, that is, copper(I) species included in the internal cavity of $\beta$-cyclodextrin covalently immobilized to superparamegnetic iron oxide nanoparticles [209]. Such material was successfully tested in the synthesis of 1-benzyl-1H-1,2,3-triazoldibenzodiazepinone derivatives encompassing two consecutive series of condensation, cyclocondensation and click reaction starting from dimedone, $o$-phenylenediamines, 4-propargyloxybenzaldehydes and benzyl azides in a $\mathrm{H}_{2} \mathrm{O} / \mathrm{EtOH}(1: 1 \mathrm{v} / \mathrm{v})$ mixture. Sarma et al. instead reported the development of copper(II) nanoparticles adsorbed on the external surface of chitosan-coated magnetic $\mathrm{Fe}_{3} \mathrm{O}_{4}$ core/shell composites, indicated as $\mathrm{CS}-\mathrm{Fe}_{3} \mathrm{O}_{4}-\mathrm{Cu}$, which was used for the synthesis of 1,4-disubstituted 1,2,3-1H-triazoles 295 by [3 + 2] Huisgen cycloaddition reactions of organic azides 293 and terminal alkynes 294, carried out in $\mathrm{CH}_{2} \mathrm{Cl}_{2}$ at room temperature for $12 \mathrm{~h}$ (Scheme 91) [210]. The $\mathrm{CS}-\mathrm{Fe}_{3} \mathrm{O}_{4}-\mathrm{Cu}$ catalyst showed good recyclability (up to four runs), while hot filtration tests confirmed the occurrence of a merely heterogeneous mechanism. Despite these appealing features, as well as the need for mild experimental conditions, the use of dichloromethane as a solvent represents a major issue in the frame of protocol sustainability. 


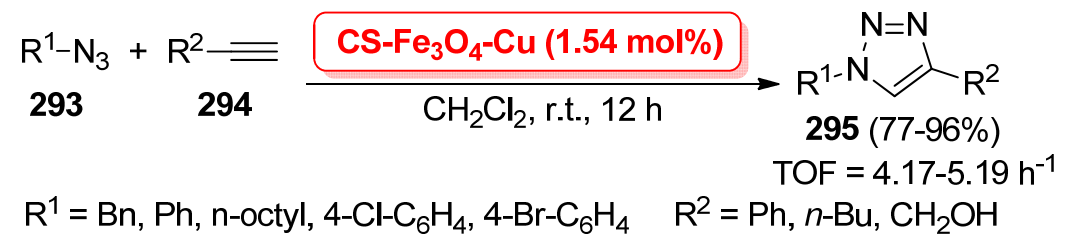

Scheme 91. Synthesis of 1,4-disubstituted 1,2,3-1H-triazoles 295 by [3 + 2] Huisgen cycloaddition reactions of organic azides 293 and terminal alkynes 294 catalyzed by CS- $\mathrm{Fe}_{3} \mathrm{O}_{4}-\mathrm{Cu}$, reported in 2015 by Sarma et al.

Besides oligo/polysaccharide-based materials, other polymers have been rarely reported as shells for the nanocomposite catalysts described in the present section. In the context of the synthesis of $N$-heterocycles, in 2014, Safari et al. reported the preparation of copper nanoparticles supported on polyethylene glycol-coated $\mathrm{Fe}_{3} \mathrm{O}_{4}$ nanocomposites $\left(\mathrm{Fe}_{3} \mathrm{O}_{4}-\mathrm{PEG}-\mathrm{Cu}\right)$. First, polyethylene glycol was functionalized with cyanuric chloride, used for covalent immobilization to $\mathrm{Fe}_{3} \mathrm{O}_{4}$ nanoparticles; then, $\mathrm{Cu}$ nanoparticles were deposited by reducing copper ammonia complexes with hydrazine on the surface of these core/shell composites, giving the final $\mathrm{Fe}_{3} \mathrm{O}_{4}-\mathrm{PEG}-\mathrm{Cu}$ [211]. Such a catalyst was found to be highly active for the synthesis of both 2,4,5-trisubstituted and 1,2,4,5-tetrasubstituted $1 \mathrm{H}$-imidazoles by multi-component reactions of 1,2-diketones, aroatic aldehydes, $\mathrm{NH}_{4} \mathrm{OAc}$ and primary amines, carried out at $110^{\circ} \mathrm{C}$ in solvent-free conditions. In 2016, Zohreh and co-workers described the immobilization of copper(II) species poly(2-dimethyaminoethyl acrylamide)-coated magnetic nanoparticles, named MNP@PDMA-Cu in the manuscript. Such a catalyst was then successfully used in the synthesis of 1,4-disubstituted 1,2,3-1Htriazoles 298 by tandem nucleophilic substitution/[3 + 2] Huisgen cycloaddition reactions of alkyl halides 296, sodium azide and terminal alkynes 297 , carried out with $0.3 \mathrm{~mol} \%$ of copper loading, in the presence of $10 \mathrm{~mol} \%$ of sodium ascorbate, at $50{ }^{\circ} \mathrm{C}$ in water as the solvent (Scheme 92) [212]. All these aspects, in combination with the good recyclability of the MNP@PDMA-Cu catalyst (up to seven times without a significant loss of activity), made this procedure highly economical and convenient from the point of view of Green Chemistry.

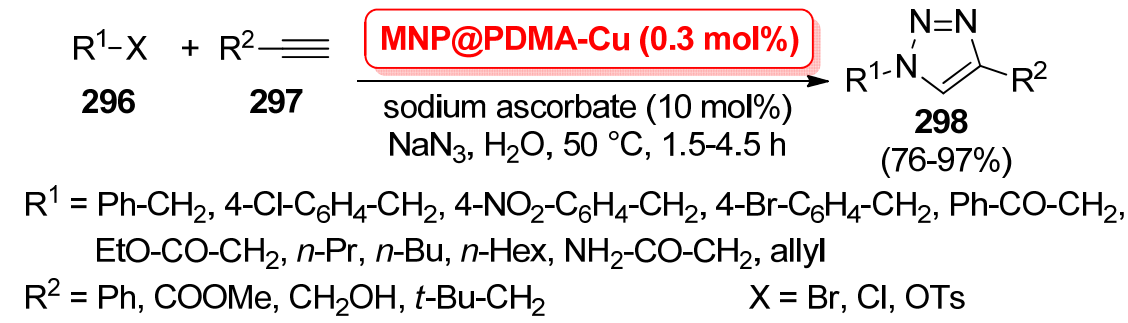

Scheme 92. Synthesis of triazoles 298 by tandem nucleophilic substitution/[3 + 2] Huisgen cycloadditions of alkyl halides 296, sodium azide and terminal alkynes 297 catalyzed by MNP@PDMA-Cu, reported in 2016 by Zohreh and co-workers.

\subsection{Metal Catalysts Supported on Metal Organic Frameworks}

Metal organic frameworks (MOFs) are a class of compounds consisting of metal ions or clusters coordinated to organic ligands to form one-, two- or three-dimensional structures. They possess ultra-high porosity, unusually large pore openings and a wide internal surface area. Therefore, it is not surprising that in the last five years, such materials have started to be successfully applied as a support for recoverable transition metal catalysts. In fact, they can be easily separated from the reaction medium by simple centrifugation and then reused many times.

The first example of the application of metal-organic frameworks as a catalyst for the synthesis of $N$-heterocyclic compounds was reported by Phan et al. in 2018, whereby they prepared $\mathrm{Cu}_{2}(\mathrm{OBA})_{2}(\mathrm{BPY})$-MOF from $\mathrm{Cu}$ (II) nitrate, $4,4^{\prime}$-oxybis(benzoic acid) (OBA) and 
4, $4^{\prime}$-bipyridine (BPY), which was then tested as a catalyst in the synthesis of aroylquinolines 301 by a one-pot domino reaction between 2-aminobenzylalcohols 299 and propiophenones 300 (2.0 equiv.), performed with $10 \mathrm{~mol} \%$ of copper loading, in the presence of pyridine (1.5 equiv.) as a ligand, TEMPO (2.0 equiv.) as an oxidizing agent, in DMF at $120^{\circ} \mathrm{C}$ for $16 \mathrm{~h}$ (Scheme 93) [213]. Unfortunately, the present protocol showed several limitations in terms of sustainability: the need for a rather large amount of the catalyst, the use of DMF as a toxic reaction medium and the presence of a supporting ligand, which could suggest the occurrence of a homogeneous mechanism (despite its rather good recyclability in eight consecutive runs).

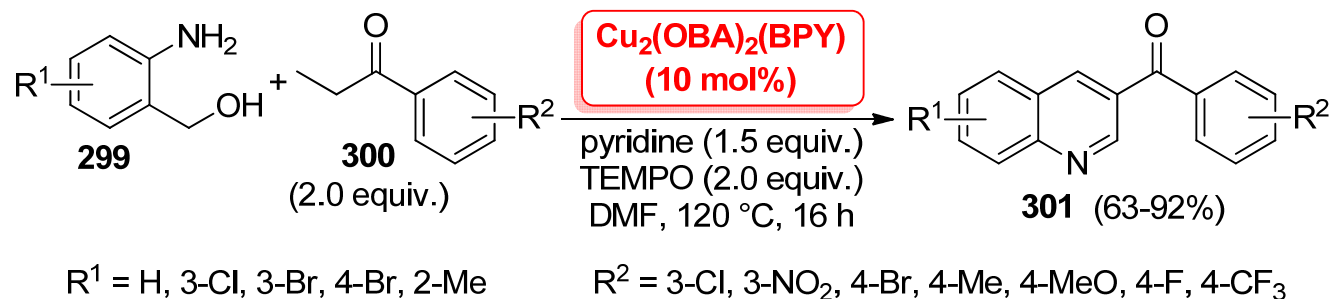

Scheme 93. Synthesis of aroylquinolines 301 by one-pot domino reaction between 2- aminobenzylalcohols 299 and propiophenones 300 catalyzed by $\mathrm{Cu}_{2}(\mathrm{OBA})_{2}(\mathrm{BPY})-\mathrm{MOF}$, reported in 2018 by Phan et al.

In 2019, Curyano and co-workers reported that the simple solid-state mixing and calcination of two different MOFs (that is, H-KUST1 and MOF-5) in the presence of copper or zinc aluminosilicates afforded highly active supported copper or zinc oxide nanoparticles [214]. Such catalysts were able to promote the synthesis of several heterocycles via C-C or $\mathrm{C}-\mathrm{N}$ bond formation, including quinolines and indoles with high turnover frequencies.

Finally, in 2020, Pal et al. applied MIL-101(Cr), which is one of the most common MOFs, to the preparation of 1,5-benzodiazepine derivatives 304 by a cyclocondensation reaction of $o$-phenylenediamines $\mathbf{3 0 2}$ and enolizable ketones 303, performed under solventfree condition at $80{ }^{\circ} \mathrm{C}$ in only $30 \mathrm{~min}$ (Scheme 94) [215]. A broad substrate scope was investigated, with diamines and ketones possessing both electron-donating and electrowithdrawing functional groups. Moreover, the MIL-101(Cr) catalyst was reused in six consecutive runs without any substantial loss in its catalytic efficiency.

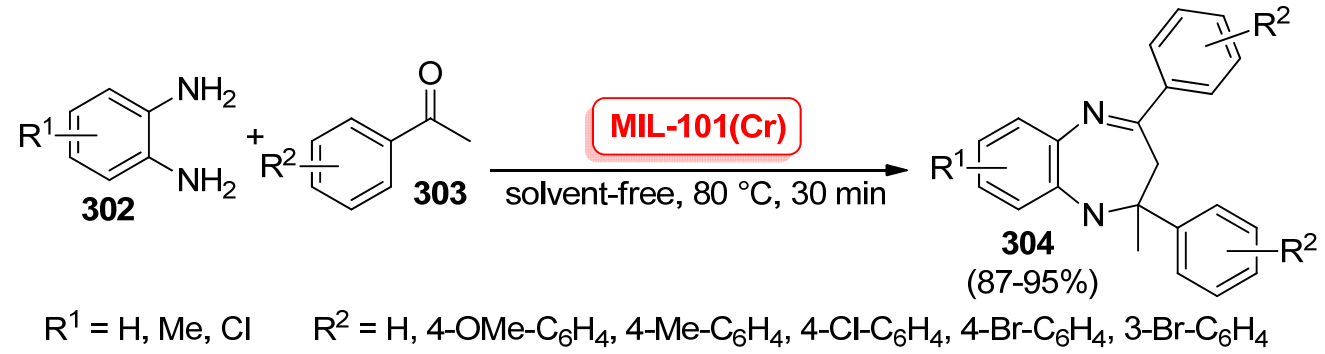

Scheme 94. Synthesis of 1,5-benzodiazepines 304 by cyclocondensation of $o$-phenylenediamines 302 and enolizable ketones 303 catalyzed by MIL-101(Cr), reported in 2020 by Pal et al.

\section{Conclusions}

In this review, we gave the first comprehensive and up-to-date overview of all the synthetic strategies for the preparation of $N$-heterocycles by supported metal catalysts. In particular, we decided to follow a systematic approach depending on the type of support. In the first part, we described metal catalysts supported on inorganic matrices; in addition to charcoal and related carbon materials, silica (including mesostructured derivatives) and metal oxides, we also described several examples of metal catalysts supported on minerals and magnetic core/inorganic shell composites. In the second part, we took into 
account metal catalysts supported on organic matrices: synthetic polymers and polymeranchored ligands on the one hand and natural biopolymers on the other. In the third part, we highlighted metal catalysts supported on hybrid inorganic-organic matrices, whose introduction in heterogeneous catalysis is definitely more recent with respect to the other two previous classes: organic ligands anchored to inorganic matrices (that is, carbon, silica, metal oxides, minerals, magnetic core/inorganic shell composite), magnetic core/organic shell composites and very recently also metal organic frameworks. In this context, a fundamental aspect is given by the reusability of such supported catalysts. Aiming to provide a critical view of this literature, here we tried to give special emphasis to their potential heterogeneity and recyclability, specifying (where possible) which studies were carried out in order to evaluate these aspects.

Although the choice of organizing such a huge amount of literature in a systematic approach depending on the type of support was, in our opinion, the most suitable for the broad community of scientists working in the field of catalysis, we still feel the need to provide with this review a very useful tool for synthetic organic chemists as well. For this reason, all the relevant information on the abovementioned catalytic systems (catalyst name, reaction type, metal loading, product yields, recycling, turnover number and/or frequency) has been organized in Table 1 below, depending on the class of $N$-heterocycle. This allowed for an easier and more immediate comparison of the critical parameters of different supported catalysts used for the synthesis of the same $N$-heterocycle.

We hope that this review will stimulate further research on the application of supported metal catalysts on the synthesis of $\mathrm{N}$-heterocyclic compounds trying to give more and more importance to all aspects in perfect agreement with the Twelve Principles of Green Chemistry.

Table 1. Supported metal catalysts for the synthesis of $N$-heterocyclic compounds: an overview of the literature cited in the previous sections, organized depending on the class of the $N$-heterocycle.

\begin{tabular}{|c|c|c|c|c|c|c|c|}
\hline $\mathrm{N}$-Heterocycle & Catalyst & Reaction Type & $\begin{array}{l}\text { Metal } \\
\text { Loading }\end{array}$ & $\begin{array}{l}\text { Product } \\
\text { Yields }\end{array}$ & Recycling & TON/TOF & Ref. \\
\hline \multirow{9}{*}{$\begin{array}{c}\text { aziridine } \\
1 H \text {-pyrrole }\end{array}$} & $\mathrm{Pd}(0)-\mathrm{AmP}-\mathrm{MCF}$ & asymmetric cascade reaction & $5 \mathrm{~mol} \%$ & $61 \%$ & 8 runs & not calculated & [180] \\
\hline & $\mathrm{Pt} / \mathrm{C}$ & dehydrogenative condensation & $0.1 \mathrm{~mol} \%$ & $63-92 \%$ & 4 runs & $\mathrm{TON}=2767$ & [46] \\
\hline & Co-Nx/C-800-AT & Paal-Knorr condensation & n.a. & $74-100 \%$ & 5 runs & not calculated & {$[56]$} \\
\hline & $\mathrm{Fe}\left(\mathrm{ClO}_{4}\right)_{3} / \mathrm{SiO}_{2}$ & Paal-Knorr condensation & $2 \mathrm{~mol} \%$ & $70-98 \%$ & 5 runs & not calculated & [63] \\
\hline & CAN-SiO 2 & $\begin{array}{l}\text { four component } \\
\text { cyclocondensation }\end{array}$ & $10 \mathrm{~mol} \%$ & $75-96 \%$ & 4 runs & not calculated & {$[66]$} \\
\hline & $\mathrm{NiFe}_{2} \mathrm{O}_{4}$ & four-component reaction & $5.0 \mathrm{~mol} \%$ & $80-96 \%$ & 9 runs & not calculated & [103] \\
\hline & $\begin{array}{c}\mathrm{CoFe}_{2} \mathrm{O}_{4} @ \mathrm{SiO}_{2-} \\
\mathrm{PrNH}_{2}-\mathrm{Mo}(\mathrm{acac})_{2}\end{array}$ & multi-component reaction & $1 \mathrm{~mol} \%$ & $48-91 \%$ & 5 runs & $\mathrm{TON}=48-91$ & [204] \\
\hline & $\begin{array}{c}\mathrm{CoFe}_{2} \mathrm{O}_{4} @ \mathrm{SiO}_{2-} \\
\mathrm{DABCO}-\mathrm{Sb}\end{array}$ & multi-component reaction & $0.5 \mathrm{~mol} \%$ & $47-93 \%$ & 5 runs & not calculated & [205] \\
\hline & $\gamma-\mathrm{Fe}_{2} \mathrm{O}_{3} @ \mathrm{SiO}_{2}-\mathrm{Sb}-\mathrm{IL}$ & Clauson-Kaas reaction & $5 \mathrm{~mol} \%$ & $81-96 \%$ & 6 runs & not calculated & [206] \\
\hline \multirow{9}{*}{$\begin{array}{l}\text { 2,5-dihydro- } 1 H \text { - } \\
\text { pyrrole }\end{array}$} & PIB-BIAN-NHC-Ru & ring closing metathesis & $1.0 \mathrm{~mol} \%$ & $95-99 \%$ & 8 runs & not calculated & [140] \\
\hline & SWNTs-Pyr-Ru & ring closing metathesis & $0.2 \mathrm{~mol} \%$ & $93-99 \%$ & 7 runs & not calculated & [150] \\
\hline & $\begin{array}{l}\text { rGO-Pyr-Ru } \\
\mathrm{SiO}_{2} \text {-supported }\end{array}$ & ring closing metathesis & $1.0 \mathrm{~mol} \%$ & $94 \%$ & 3 runs & not calculated & [151] \\
\hline & $\begin{array}{c}\text { Grubbs-Hoveyda Ru } \\
\text { catalyst }\end{array}$ & ring closing metathesis & $2.5 \mathrm{~mol} \%$ & $98 \%$ & 2 runs & $\mathrm{TON}=169$ & [163] \\
\hline & $\begin{array}{c}\mathrm{SiO}_{2} \text {-supported Grubbs } \\
\text { Ru catalyst }\end{array}$ & ring closing metathesis & $0.4 \mathrm{~mol} \%$ & $84-85 \%$ & n.a. & not calculated & [164] \\
\hline & $\begin{array}{c}\mathrm{SiO}_{2} \text {-supported } \\
\text { Heoveyda-Grubbs Ru } \\
\text { complex }\end{array}$ & ring closing metathesis & $2.0 \mathrm{~mol} \%$ & $33-94 \%$ & 3 runs & not calculated & [165] \\
\hline & $\begin{array}{c}\text { SBA-15-supported } \\
\text { Hoveyda-type Ru catalyst }\end{array}$ & ring closing metathesis & $0.1 \mathrm{~mol} \%$ & $69-89 \%$ & 23 runs & $\mathrm{TON}=3450-17,400$ & [173] \\
\hline & $\begin{array}{c}\mathrm{Pd}(0)-\mathrm{AmP}^{-M C F} \\
\mathrm{Fe}_{3} \mathrm{O}_{4} @ \mathrm{SiO}_{2} \text {-supported }\end{array}$ & asymmetric cascade reaction & $3 \mathrm{~mol} \%$ & $53-84 \%$ & 8 runs & not calculated & [179] \\
\hline & $\begin{array}{c}\text { Grubbs-Hoveyda- type Ru } \\
\text { catalyst }\end{array}$ & ring closing metathesis & $0.85 \mathrm{~mol} \%$ & $91-99 \%$ & 6 runs & not calculated & [202] \\
\hline
\end{tabular}


Table 1. Cont.

\begin{tabular}{|c|c|c|c|c|c|c|c|}
\hline $\mathrm{N}$-Heterocycle & Catalyst & Reaction Type & $\begin{array}{l}\text { Metal } \\
\text { Loading }\end{array}$ & $\begin{array}{l}\text { Product } \\
\text { Yields }\end{array}$ & Recycling & TON/TOF & Ref. \\
\hline \multirow[t]{3}{*}{ 1H-pyrazole } & $\begin{array}{l}\mathrm{Pd} / \mathrm{C}+\mathrm{K}-10 \\
\text { montmorillonite }\end{array}$ & cyclization/dehydrogenation & $10 \mathrm{~mol} \%$ & $80-98 \%$ & n.a. & not calculated & [38] \\
\hline & $\mathrm{CuO} / \mathrm{ZrO}_{2}$ & multicomponent reaction & $0.5 \mathrm{~mol} \%$ & $88-92 \%$ & 5 runs & not calculated & [92] \\
\hline & $\mathrm{Fe}_{3} \mathrm{O}_{4} @ \mathrm{Si} @ \mathrm{MoO}_{2}$ & multi-component reaction & $\begin{array}{c}20 \\
\mathrm{mg} / \mathrm{mmol}\end{array}$ & $85-95 \%$ & 8 runs & not calculated & [203] \\
\hline \multirow[t]{11}{*}{$1 H$-imidazole } & $\mathrm{Bi}_{2} \mathrm{O}_{3}-\mathrm{ZnO} /$ fly ash & condensation/cyclization & 5 wt. $\%$ & $89-98 \%$ & 4 runs & not calculated & [53] \\
\hline & $\mathrm{SbCl}_{3} / \mathrm{SiO}_{2}$ & $\begin{array}{l}\text { multi-component } \\
\text { cyclocondensation }\end{array}$ & $44 \mathrm{~mol} \%$ & $58-95 \%$ & n.a. & not calculated & [64] \\
\hline & $\mathrm{SbCl}_{3} / \mathrm{SiO}_{2}$ & $\begin{array}{l}\text { multi-component } \\
\text { cyclocondensation }\end{array}$ & $44 \mathrm{~mol} \%$ & $76-97 \%$ & 5 runs & not calculated & {$[65]$} \\
\hline & $\mathrm{CuFe}_{2} \mathrm{O}_{4}$ & condensation/cyclization & $10 \mathrm{~mol} \%$ & $84-91 \%$ & 6 runs & not calculated & [100] \\
\hline & $\mathrm{CuFe}_{2} \mathrm{O}_{4}$ & three-component reaction & $\begin{array}{c}50 \\
\mathrm{mg} / \mathrm{mmol}\end{array}$ & $92-96 \%$ & 5 runs & not calculated & [101] \\
\hline & $\mathrm{CoFe}_{2} \mathrm{O}_{4}$ & three-component reaction & $\begin{array}{l}50 \\
\mathrm{mg} / \mathrm{mmol}\end{array}$ & $94-98 \%$ & 5 runs & not calculated & [101] \\
\hline & Ni-bentonite@ferrite & condensation/cyclization & $\begin{array}{l}37 \\
\mathrm{mg} / \mathrm{mmol}\end{array}$ & $81-92 \%$ & 8 runs & not calculated & [117] \\
\hline & $\begin{array}{c}\text { Zr-CAP-SG } \\
\text { SBA-15-DMBA-Cu(I) }\end{array}$ & $\begin{array}{l}\text { condensation } \\
\text { condensation/cylization }\end{array}$ & $\begin{array}{l}10 \mathrm{wt} . \% \\
0.7 \mathrm{~mol} \%\end{array}$ & $\begin{array}{l}71-87 \% \\
88-98 \%\end{array}$ & $\begin{array}{l}5 \text { runs } \\
5 \text { runs }\end{array}$ & $\begin{array}{l}\text { not calculated } \\
\text { not calculated }\end{array}$ & $\begin{array}{l}{[166]} \\
{[167]}\end{array}$ \\
\hline & $\mathrm{Fe}_{3} \mathrm{O}_{4}$-DOPA-Cu & $\begin{array}{l}\text { tandem } \mathrm{S}_{\mathrm{N}} 2 /[3+2] \\
\text { cycloaddition }\end{array}$ & $\begin{array}{l}30 \\
\mathrm{mg} / \mathrm{mmol}\end{array}$ & $90-96 \%$ & 6 runs & not calculated & [184] \\
\hline & cellulose $/ \gamma-\mathrm{Fe}_{2} \mathrm{O}_{3} / \mathrm{Ag}$ & multi-component reaction & $\begin{array}{c}15 \\
\mathrm{mg} / \mathrm{mmol}\end{array}$ & $82-95 \%$ & 5 runs & not calculated & [208] \\
\hline & $\mathrm{Fe}_{3} \mathrm{O}_{4}-\mathrm{PEG}-\mathrm{Cu}$ & multi-component reaction & $10 \mathrm{~mol} \%$ & $88-98 \%$ & 6 runs & not calculated & [211] \\
\hline \multirow[t]{32}{*}{ 1,2,3-1H-triazole } & $\mathrm{Pd}-\mathrm{Cu} / \mathrm{C}$ & $\begin{array}{c}\text { Sonogashira/desilylation/ } \\
{[3+2] \text { cycloaddition }}\end{array}$ & $5.0 \mathrm{~mol} \%$ & $52-76 \%$ & 1 run & not calculated & [40] \\
\hline & $\mathrm{Cu} / \mathrm{C}$ & {$[3+2]$ cycloaddition } & $5.0 \mathrm{~mol} \%$ & $92-99 \%$ & 3 runs & not calculated & [41] \\
\hline & $\mathrm{Cu} / \mathrm{C}$ & $\begin{array}{l}\text { diazo transfer } /[3+2] \\
\text { cycloaddition }\end{array}$ & $12 \mathrm{~mol} \%$ & $35-99 \%$ & 2 runs & not calculated & {$[42]$} \\
\hline & CuNPs/C & $\begin{array}{l}\text { three-component }[3+2] \\
\text { cycloaddition }\end{array}$ & $0.5 \mathrm{~mol} \%$ & $85-99 \%$ & 2 runs & not calculated & [43] \\
\hline & CuNPs/C & $\begin{array}{l}\text { three-component }[3+2] \\
\text { cycloaddition }\end{array}$ & $0.5 \mathrm{~mol} \%$ & $64-99 \%$ & 5 runs & not calculated & {$[44]$} \\
\hline & TRGO/Cu(I) & {$[3+2]$ cycloaddition } & $2 \mathrm{~mol} \%$ & $80-99 \%$ & 4 runs & not calculated & [48] \\
\hline & Cu NPs-MCN & $\begin{array}{l}\text { three-component }[3+2] \\
\text { cycloaddition }\end{array}$ & $0.5 \mathrm{~mol} \%$ & $78-98 \%$ & 8 runs & not calculated & {$[54]$} \\
\hline & CDSCS & {$[3+2]$ cycloaddition } & $0.05 \mathrm{~mol} \%$ & $80-93 \%$ & 5 runs & not calculated & {$[60]$} \\
\hline & CPSi & $\begin{array}{l}\text { three-component reaction } \\
\text { nucleophilic }\end{array}$ & $0.64 \mathrm{~mol} \%$ & $80-95 \%$ & 5 runs & $\mathrm{TON}=38-47.5$ & [72] \\
\hline & $\mathrm{Cu} / \mathrm{Al}_{2} \mathrm{O}_{3}$ & $\begin{array}{l}\text { substitution } /[3+2] \\
\text { cycloaddition }\end{array}$ & $10 \mathrm{~mol} \%$ & $70-96 \%$ & 8 runs & not calculated & [74] \\
\hline & $\mathrm{CuI} / \mathrm{Al}_{2} \mathrm{O}_{3}$ & $\begin{array}{l}\text { azide formation } /[3+2] \\
\text { cycloaddition }\end{array}$ & $3 \mathrm{~mol} \%$ & $70-98 \%$ & 8 runs & $\begin{array}{c}\mathrm{TON}=495 \\
\mathrm{TOF}=1.37 \mathrm{~s}^{-1}\end{array}$ & [75] \\
\hline & $\mathrm{CuO} / \mathrm{ZnO}$ & $\begin{array}{c}\text { azide formation } /[3+2] \\
\text { cycloaddition }\end{array}$ & $22 \mathrm{~mol} \%$ & $89-92 \%$ & 5 runs & not calculated & [96] \\
\hline & $\mathrm{CuFe}_{2} \mathrm{O}_{4}$ & $\begin{array}{l}\text { nucleophilic substitution/ } \\
{[3+2] \text { cycloaddition }}\end{array}$ & $5.0 \mathrm{~mol} \%$ & $74-93 \%$ & 4 runs & not calculated & {$[98]$} \\
\hline & $\mathrm{CuFe}_{2} \mathrm{O}_{4}$ & multicomponent reaction & $10 \mathrm{~mol} \%$ & $74-92 \%$ & 3 runs & not calculated & [99] \\
\hline & clay-Cu(II) & $\begin{array}{l}\text { azidonation } /[3+2] \\
\text { cycloaddition }\end{array}$ & $10 \mathrm{~mol} \%$ & $83-98 \%$ & 5 runs & not calculated & [106] \\
\hline & clay-Cu(II) & {$[3+2]$ cycloaddition } & $5.0 \mathrm{~mol} \%$ & $92-96 \%$ & 5 runs & not calculated & [107] \\
\hline & $\mathrm{CuBr} / \mathrm{GO} @ \mathrm{Fe}_{3} \mathrm{O}_{4}$ & $\begin{array}{l}\text { azide formation } /[3+2] \\
\text { cycloaddition }\end{array}$ & $5.0 \mathrm{~mol} \%$ & $88-98 \%$ & 6 runs & not calculated & [112] \\
\hline & $\mathrm{Cu} / \mathrm{CNT} @ \mathrm{CuFe}_{2} \mathrm{O}_{4}$ & $\begin{array}{l}\text { azide formation } /[3+2] \\
\text { cycloaddition }\end{array}$ & $0.4 \mathrm{~mol} \%$ & $57-92 \%$ & 4 runs & not calculated & [115] \\
\hline & $\mathrm{SMI}-\mathrm{Cu}(\mathrm{I})$ & $\begin{array}{l}\text { azide formation } /[3+2] \\
\text { cycloaddition }\end{array}$ & $35.1 \mathrm{~mol} \%$ & $71-91 \%$ & 5 runs & not calculated & [120] \\
\hline & nano- $\mathrm{Cu}_{2} \mathrm{O}-\mathrm{MFR}$ & {$[3+2]$ cycloaddition } & $\begin{array}{l}0.072 \\
\mathrm{~mol} \%\end{array}$ & $80-93 \%$ & 5 runs & not calculated & [128] \\
\hline & Cu-HMOP & azidation/[3 +2$]$ cycloaddition & 10 wt. $\%$ & $63-99 \%$ & 5 runs & not calculated & [129] \\
\hline & PS-L-Ru & $\begin{array}{l}\text { azide formation } /[3+2] \\
\text { cycloaddition }\end{array}$ & $2.1 \mathrm{~mol} \%$ & $76-100 \%$ & 6 runs & $\mathrm{TON}=804-950$ & [133] \\
\hline & Chitosan-Phen-Cu(I) & {$[3+2]$ cycloaddition } & $0.1 \mathrm{~mol} \%$ & $64-99 \%$ & 4 runs & not calculated & [134] \\
\hline & TG-TBTA-CuPF 6 & {$[3+2]$ cycloaddition } & $1.0 \mathrm{~mol} \%$ & $83-99 \%$ & 10 runs & not calculated & [135] \\
\hline & $\begin{array}{l}\text { PS-TBTA } \cdot \mathrm{CuX}_{1-2} \\
\text { beads and flow }\end{array}$ & {$[3+2]$ cycloaddition } & $\begin{array}{c}0.6-20 \\
\mathrm{~mol} \%\end{array}$ & Up to $99 \%$ & $\begin{array}{l}\text { up to } 8 \\
\text { runs }\end{array}$ & $\mathrm{TON}=188-304$ & [136] \\
\hline & $\mathrm{Chit}_{-\mathrm{CuSO}}$ & {$[3+2]$ cycloaddition } & $0.4 \mathrm{~mol} \%$ & $93-99 \%$ & 5 runs & not calculated & [146] \\
\hline & $\begin{array}{c}\text { AMWCNTs-O-Cu(II)- } \\
\text { PhTPY }\end{array}$ & {$[3+2]$ cycloaddition } & $2 \mathrm{~mol} \%$ & $74-97 \%$ & 5 runs & not calculated & [148] \\
\hline & CRGO-Ima-Cu(I) & {$[3+2]$ cycloaddition } & $2.0 \mathrm{~mol} \%$ & $85-99 \%$ & 10 runs & not calculated & [152] \\
\hline & $\begin{array}{l}\text { SiFK IAd/Cu(I) } \\
\text { SiNPIAd/Cu(I) }\end{array}$ & {$[3+2]$ cycloaddition } & $1.0 \mathrm{~mol} \%$ & $56-68 \%$ & 3 runs & not calculated & [153] \\
\hline & Cu(II)-ID@Silica & $\begin{array}{l}\text { azidation } /[3+2] \text { cycloaddition } \\
\text { four component } \mathrm{C}-\mathrm{H} \text { bond }\end{array}$ & $1.0 \mathrm{~mol} \%$ & $90-98 \%$ & 12 runs & not calculated & [154] \\
\hline & BS-Cu(II)@SiO 2 & $\begin{array}{l}\text { activation } /[3+2] \\
\text { cycloaddition }\end{array}$ & $5.0 \mathrm{~mol} \%$ & $87-94 \%$ & 7 runs & not calculated & [155] \\
\hline & CuNPs-PEI $/ \mathrm{SiO}_{2}$ & {$[3+2]$ cycloaddition } & $0.05 \mathrm{~mol} \%$ & $60-98 \%$ & 3 runs & not calculated & [157] \\
\hline
\end{tabular}


Table 1. Cont.

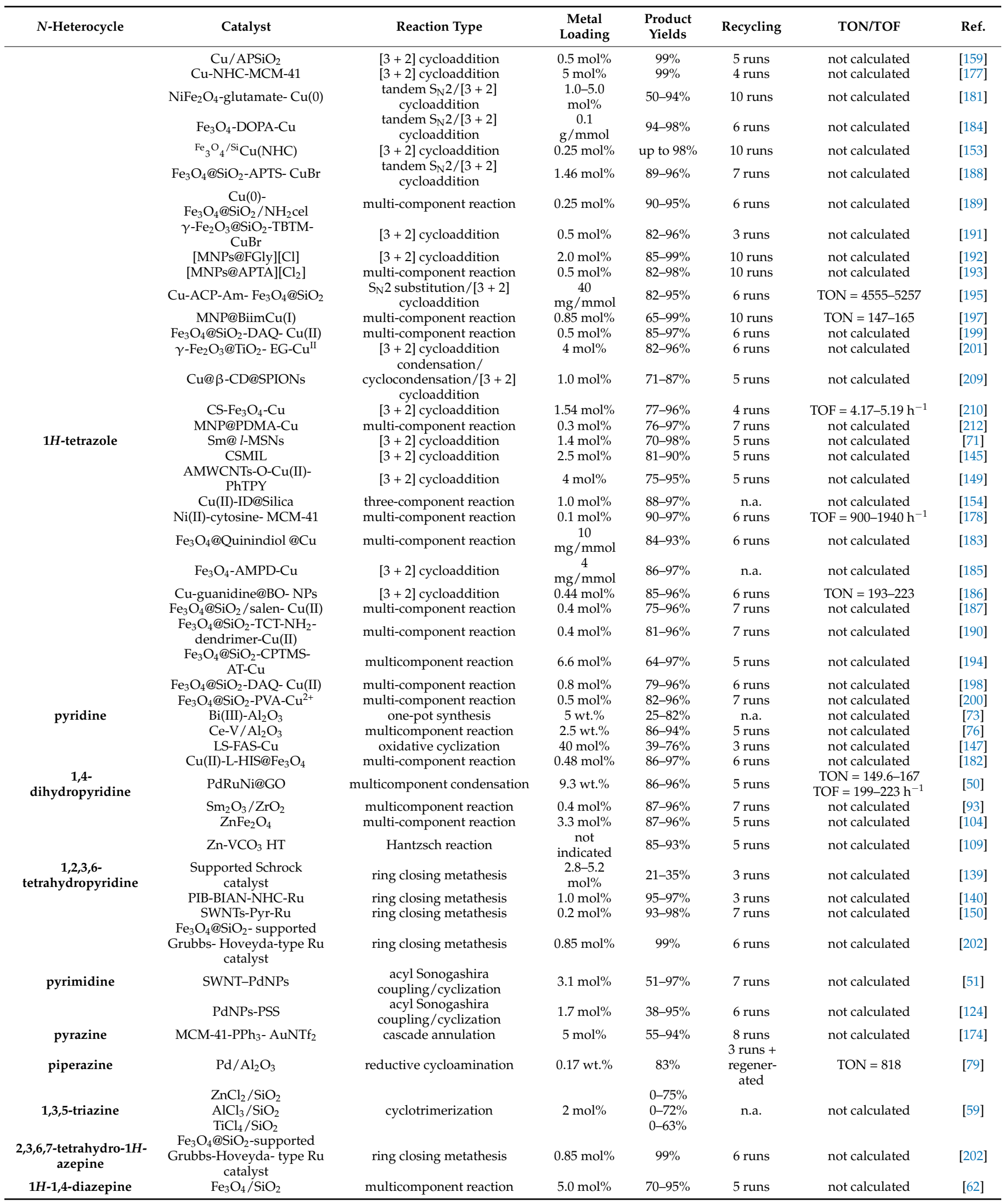


Table 1. Cont.

\begin{tabular}{|c|c|c|c|c|c|c|c|}
\hline $\mathrm{N}$-Heterocycle & Catalyst & Reaction Type & $\begin{array}{l}\text { Metal } \\
\text { Loading }\end{array}$ & $\begin{array}{l}\text { Product } \\
\text { Yields }\end{array}$ & Recycling & TON/TOF & Ref. \\
\hline \multirow[t]{13}{*}{$1 H$-indole } & $\mathrm{Pd} / \mathrm{C}$ & $\begin{array}{c}\text { Sonogashira/intramolecular } \\
\text { heteroannulation }\end{array}$ & $1.0 \mathrm{~mol} \%$ & $72 \%$ & 3 runs & not calculated & [37] \\
\hline & $\mathrm{Pt} / \mathrm{Al}_{2} \mathrm{O}_{3}+\mathrm{ZnO}$ & dehydrogenative condensation & $\begin{array}{c}1.7 \mathrm{~mol}^{\%} \\
\mathrm{Pt} \\
4.5 \mathrm{~mol} \% \\
\mathrm{Zn}\end{array}$ & $30-99 \%$ & n.a. & not calculated & [80] \\
\hline & $\mathrm{Ru} / \mathrm{CeO}_{2}$ & $\begin{array}{l}\text { intramolecular } \\
\text { dehydrogenative } \\
\text { heterocyclization }\end{array}$ & $2.5 \mathrm{~mol} \%$ & $84 \%$ & 2 runs & not calculated & [91] \\
\hline & $\mathrm{Pt} / \mathrm{Nb}_{2} \mathrm{O}_{5}$ & $\begin{array}{c}\text { dehydrogenative } \\
N \text {-heterocyclization }\end{array}$ & $0.2 \mathrm{~mol} \%$ & $76 \%$ & 3 runs & $\mathrm{TON}=380$ & [97] \\
\hline & $\mathrm{Au} / \mathrm{Fe}_{2} \mathrm{O}_{3}$ & hydrogenation/hydroamination & $2.3 \mathrm{~mol} \%$ & $20-94 \%$ & 1 run & not calculated & [102] \\
\hline & CuAl-HT & $\begin{array}{c}\text { intramolecular } \\
\text { dehydrogenative } \\
N \text {-heterocyclization }\end{array}$ & $\begin{array}{c}0.2 \\
\mathrm{~g} / \mathrm{mmol}\end{array}$ & $94-100 \%$ & 6 runs & not calculated & [108] \\
\hline & $\mathrm{Pt} / \mathrm{HBEA}$ & $\begin{array}{c}\text { dehydrogenative } \\
N \text {-heterocyclization }\end{array}$ & $1.0 \mathrm{~mol} \%$ & $68-90 \%$ & 3 runs & $\mathrm{TON}=450$ & [97] \\
\hline & Pd@PS & $\begin{array}{c}\text { domino decarboxylative } \\
\text { coupling/5-exo-dig cyclization }\end{array}$ & $3 \mathrm{~mol} \%$ & $63-76 \%$ & 5 runs & not calculated & [121] \\
\hline & LS-FAS-Cu & multi-component reaction & $20 \mathrm{~mol} \%$ & $37-80 \%$ & 3 runs & not calculated & [147] \\
\hline & $\begin{array}{c}\mathrm{SiO}_{2}-\left[\left(\mathrm{IPr}_{\mathrm{R}}\right) \mathrm{Au}\right] \mathrm{Cl} \\
\mathrm{SiO}_{2}-\left[\left(\mathrm{IAdPr}_{\mathrm{R}}\right) \mathrm{Au}\right] \mathrm{Cl}\end{array}$ & cyclization & $1.0 \mathrm{~mol} \%$ & $99 \%$ & 5 runs & not calculated & [160] \\
\hline & {$\left[\mathrm{PdCl}_{2}(\mathrm{NCPh})_{2}\right]$} & cyclization & $5.0 \mathrm{~mol} \%$ & $89-96 \%$ & 4 runs & not calculated & [161] \\
\hline & SBA-15-anchored $\mathrm{PdCl}_{2} \mathrm{~L}_{2}$ & Larock synthesis & $1.0 \mathrm{~mol} \%$ & $80-85 \%$ & 4 runs & not calculated & [172] \\
\hline & CuOMOFY & multi-component reaction & $0.7 \mathrm{~mol} \%$ & $91-94 \%$ & n.a. & $\mathrm{TOF}=67-104 \mathrm{~h}^{-1}$ & [214] \\
\hline indolizine & CuNPs/C & multicomponent reaction & $0.5 \mathrm{~mol} \%$ & $59-93 \%$ & 1 run & not calculated & {$[45]$} \\
\hline isoindoline & Pd/Smopex ${ }^{\circledR}-234$ & $\begin{array}{l}\text { cyclocarbonylative } \\
\text { Sonogashira coupling }\end{array}$ & $0.4 \mathrm{~mol} \%$ & $75 \%$ & n.a. & not calculated & [125] \\
\hline \multirow[t]{16}{*}{$\begin{array}{l}1 H \text {-benzo }[d] \\
\text { imidazole }\end{array}$} & $\begin{array}{l}\mathrm{Pd} / \mathrm{C}+\mathrm{K}-10 \\
\text { montmorillonite }\end{array}$ & $\begin{array}{l}\text { transfer hydrogenation/ con- } \\
\text { densation/dehydrogenation }\end{array}$ & $5.0 \mathrm{~mol} \%$ & $72-84 \%$ & n.a. & not calculated & {$[39]$} \\
\hline & $\mathrm{Cu} @ \mathrm{U}-\mathrm{g}-\mathrm{C}_{3} \mathrm{~N}_{4}$ & $\mathrm{CO}_{2}$-fixation/cyclization & $1.9 \mathrm{~mol} \%$ & $62-92 \%$ & 5 runs & not calculated & [55] \\
\hline & $\mathrm{Ni}_{2} \mathrm{P} / N, P-\mathrm{C}-800$ & $\begin{array}{c}\text { oxidative cross- } \\
\text { dehydrogenative coupling }\end{array}$ & $7.5 \mathrm{~mol} \%$ & $73-93 \%$ & 5 runs & not calculated & {$[58]$} \\
\hline & $\mathrm{CuO}-\mathrm{np} / \mathrm{SiO}_{2}$ & condensation/dehydrogenation & $10 \mathrm{~mol} \%$ & $76-93 \%$ & 5 runs & not calculated & [61] \\
\hline & Yb@l-MSNs & $\begin{array}{l}\text { condensation/dehydrogenation } \\
\text { isomerization/imine }\end{array}$ & $2.25 \mathrm{~mol} \%$ & $80-92 \%$ & 5 runs & $\mathrm{TON}=27-34$ & [70] \\
\hline & $\mathrm{Pd} / \mathrm{Al}_{2} \mathrm{O}_{3}$ & $\begin{array}{l}\text { formation/ring } \\
\text { closing/dehydrogenation }\end{array}$ & $2.5 \mathrm{~mol} \%$ & $50 \%$ & 4 runs & not calculated & [78] \\
\hline & $\mathrm{Au} / \mathrm{TiO}_{2}$ & $\begin{array}{l}\text { hydrogenation } / \mathrm{CO}_{2-}^{-} \\
\text {fixation/cyclization }\end{array}$ & $1.0 \mathrm{~mol} \%$ & $28-86 \%$ & 1 run & not calculated & [81] \\
\hline & $\mathrm{Au} / \mathrm{TiO}_{2}$ & $\begin{array}{l}\text { two hydrogen- transfer } \\
\text { reaction }\end{array}$ & $2.0 \mathrm{~mol} \%$ & $70-81 \%$ & 7 runs & not calculated & [82] \\
\hline & $\begin{array}{l}\mathrm{Au} / \mathrm{TiO}_{2} \\
\mathrm{Au} / \mathrm{CeO}_{2}\end{array}$ & $\begin{array}{l}\text { condensation/dehydrogenation } \\
\text { one-pot four step protocol }\end{array}$ & $\begin{array}{l}1 \mathrm{~mol} \% \\
0.5 \mathrm{~mol} \%\end{array}$ & $\begin{array}{l}51-99 \% \\
13-91 \%\end{array}$ & $\begin{array}{l}5 \text { runs } \\
2 \text { runs }\end{array}$ & $\begin{array}{l}\text { not calculated } \\
\text { not calculated }\end{array}$ & $\begin{array}{l}{[84]} \\
{[88]}\end{array}$ \\
\hline & $\mathrm{Co} / \mathrm{MnO}$ & condensation/dehydrogenation & $\begin{array}{c}100 \\
\mathrm{mg} / \mathrm{mmol}\end{array}$ & $89-97 \%$ & 4 runs & not calculated & [94] \\
\hline & $\begin{array}{c}\mathrm{FeCl}_{3} / \mathrm{PANI} \\
\text { PANI@Au:CuO }\end{array}$ & $\begin{array}{l}\text { condensation/dehydrogenation } \\
\text { amination/azidation/annulation }\end{array}$ & $\begin{array}{l}19 \mathrm{wt} \% \\
1.0 \mathrm{~mol} \%\end{array}$ & $\begin{array}{l}70-97 \% \\
61-87 \%\end{array}$ & $\begin{array}{l}4 \text { runs } \\
7 \text { runs }\end{array}$ & $\begin{array}{l}\text { not calculated } \\
\text { not calculated }\end{array}$ & $\begin{array}{l}{[122]} \\
{[123]}\end{array}$ \\
\hline & Cu-NPs@COF & $\mathrm{CO}_{2}$-fixation/cyclization & $\begin{array}{c}30 \\
\mathrm{mg} / \mathrm{mmol}\end{array}$ & $71-96 \%$ & 6 runs & not calculated & [130] \\
\hline & $\begin{array}{l}\text { PS-Zn(II)SALTETA } \\
\text { Cu(II)-ID@Silica }\end{array}$ & $\begin{array}{l}\mathrm{CO}_{2} \text {-fixation/cyclization } \\
\text { one pot cyclization }\end{array}$ & $\begin{array}{l}3.0 \mathrm{~mol} \% \\
5.0 \mathrm{~mol} \%\end{array}$ & $\begin{array}{l}68-95 \% \\
70-84 \%\end{array}$ & $\begin{array}{l}7 \text { runs } \\
\text { n.a. }\end{array}$ & $\begin{array}{l}\text { not calculated } \\
\text { not calculated }\end{array}$ & $\begin{array}{l}{[138]} \\
{[154]}\end{array}$ \\
\hline & BS-Cu(II)@SiO 2 & $\begin{array}{l}\text { condensation/cyclization/ } \\
\text { aromatization }\end{array}$ & $5.0 \mathrm{~mol} \%$ & $87-94 \%$ & 5 runs & not calculated & [155] \\
\hline & Cu@QSSi & multi-component reaction & $5.0 \mathrm{~mol} \%$ & $82-91 \%$ & 7 runs & not calculated & [156] \\
\hline & $\mathrm{Cu}(\mathrm{II})-\mathrm{TD} @ \mathrm{nSiO}_{2}$ & condensation/cyclization & $0.3 \mathrm{~mol} \%$ & $88-97 \%$ & 8 runs & $\mathrm{TOF}=178-856 \mathrm{~h}^{-1}$ & [158] \\
\hline \multirow[t]{2}{*}{ benzo $[d]$ oxazole } & $\mathrm{CuO}-\mathrm{np} / \mathrm{SiO}_{2}$ & condensation/dehydrogenation & $10 \mathrm{~mol} \%$ & $72-85 \%$ & 5 runs & not calculated & [61] \\
\hline & $\mathrm{Au} / \mathrm{TiO}_{2}$ & two hydrogen-transfer reaction & $2.0 \mathrm{~mol} \%$ & $55-91 \%$ & 7 runs & not calculated & [82] \\
\hline \multirow{10}{*}{$\begin{array}{c}\text { benzo }[d] \text { thiazole } \\
1 H \text {-pyrrolo }[2,3-b] \\
\text { pyridine } \\
\text { imidazo }[1,2-a] \\
\text { pyridine }\end{array}$} & $\mathrm{CuO}-\mathrm{np} / \mathrm{SiO}_{2}$ & condensation/dehydrogenation & $10 \mathrm{~mol} \%$ & $80-88 \%$ & 5 runs & not calculated & [61] \\
\hline & $\mathrm{Pd}(0)-\mathrm{NaY}$ & heteroannulation & $5.0 \mathrm{~mol} \%$ & $40-71 \%$ & 4 runs & not calculated & [110] \\
\hline & $\mathrm{CuO} / \mathrm{rGO}$ & three-component reaction & $2.8 \mathrm{~mol} \%$ & $86-95 \%$ & 5 runs & $\begin{array}{c}\text { TOF }= \\
29.7-35.7 \mathrm{~h}^{-1}\end{array}$ & [49] \\
\hline & $\mathrm{BF}_{3} / \mathrm{MCM}-41$ & $\begin{array}{l}\text { multi-component } \\
\text { cyclocondensation }\end{array}$ & 20 wt. $\%$ & $75-95 \%$ & 5 runs & $\mathrm{TON}=7.14$ & [69] \\
\hline & $\mathrm{CuCl}_{2} /$ nano- $\mathrm{TiO}_{2}$ & condensation/dehydrogenation & $0.8 \mathrm{~mol} \%$ & $55-92 \%$ & 4 runs & not calculated & [85] \\
\hline & $\mathrm{Cu} / \mathrm{H}-\mathrm{OMS}-2$ & $\begin{array}{l}\text { Michael addition/ oxidative } \\
\text { cyclization }\end{array}$ & $0.7 \mathrm{~mol} \%$ & $39-88 \%$ & 3 runs & not calculated & {$[95]$} \\
\hline & $\mathrm{Cu} / \mathrm{CNT} @ \mathrm{CoFe}_{2} \mathrm{O}_{4}$ & multi-component reaction & $5.0 \mathrm{~mol} \%$ & $80-95 \%$ & 8 runs & not calculated & [114] \\
\hline & $\begin{array}{l}\mathrm{n}-\mathrm{SiO}_{2}-\mathrm{AP}-\mathrm{CC}- \\
\text { bis(Amp)-Pd }\end{array}$ & multi-component reaction & $0.2 \mathrm{~mol} \%$ & $51-89 \%$ & 6 runs & not calculated & [162] \\
\hline & $\mathrm{MCM}-41-\mathrm{PPh}_{3}-\mathrm{AuCl}$ & cascade annulation & $3 \mathrm{~mol} \%$ & $58-89 \%$ & 8 runs & not calculated & [175] \\
\hline & MNP@BiimCu(I) & multi-component reaction & $1.2 \mathrm{~mol} \%$ & $65-95 \%$ & 10 runs & $\mathrm{TON}=54-79$ & [196] \\
\hline
\end{tabular}


Table 1. Cont.

\begin{tabular}{|c|c|c|c|c|c|c|c|}
\hline$N$-Heterocycle & Catalyst & Reaction Type & $\begin{array}{l}\text { Metal } \\
\text { Loading }\end{array}$ & $\begin{array}{l}\text { Product } \\
\text { Yields }\end{array}$ & Recycling & TON/TOF & Ref. \\
\hline $\begin{array}{l}\text { imidazo }[2,1-b] \\
\text { pyridine }\end{array}$ & PS-en-Pd(II) & $\begin{array}{c}\text { Sonogashira } \\
\text { coupling/heteroannulation }\end{array}$ & $3 \mathrm{~mol} \%$ & $62-90 \%$ & 5 runs & not calculated & [132] \\
\hline $\begin{array}{l}\text { imidazo }[1,2-a] \\
\text { pyrimidine }\end{array}$ & $\mathrm{Cu} / \mathrm{H}-\mathrm{OMS}-2$ & $\begin{array}{l}\text { Michael addition/oxidative } \\
\text { cyclization }\end{array}$ & $0.7 \mathrm{~mol} \%$ & $25-81 \%$ & 3 runs & not calculated & [95] \\
\hline $\begin{array}{l}\text { pyrazolo[3,4- } b] \\
\text { pyridine }\end{array}$ & $\mathrm{FeCl}_{3} / \mathrm{Al}_{2} \mathrm{O}_{3}$ & multicomponent reaction & $20 \mathrm{~mol} \%$ & $80-86 \%$ & n.a. & not calculated & [77] \\
\hline $\begin{array}{l}\text { 1,2,3-triazolo } \\
{[1,5-a] \text { pyridine }}\end{array}$ & $\mathrm{MCM}-41-2 \mathrm{~N}-\mathrm{Cu}(\mathrm{OAc})_{2}$ & one-pot reaction & $5 \mathrm{~mol} \%$ & $55-96 \%$ & 7 runs & not calculated & [176] \\
\hline $9 H$-purine & $\mathrm{Ag} / \mathrm{SiO}_{2}$ & condensation/dehydrogenation & $3 \mathrm{~mol} \%$ & $89-94 \%$ & 5 runs & not calculated & [67] \\
\hline \multirow[t]{6}{*}{ quinoline } & $\mathrm{CoO} / \mathrm{MWCNTs}$ & Friedländer condensation & $\begin{array}{c}0.86-4.2 \\
\mathrm{~mol} \%\end{array}$ & $51-86 \%$ & 5 runs & $\mathrm{TOF}=0.36-0.55 \mathrm{~s}^{-1}$ & {$[52]$} \\
\hline & $\mathrm{AuNPs} / \mathrm{SiO}_{2}$ & oxidative cyclization & $\begin{array}{c}5 \mathrm{~mol} \% \\
1.7 \mathrm{~mol} \%\end{array}$ & $17-95 \%$ & 7 runs & not calculated & [68] \\
\hline & $\mathrm{Pt} / \mathrm{Al}_{2} \mathrm{O}_{3}+\mathrm{ZnO}$ & dehydrogenative condensation & $\begin{array}{l}\mathrm{Pt} \\
4.5 \mathrm{~mol} \% \\
\mathrm{Zn}\end{array}$ & $18-62 \%$ & n.a. & not calculated & [80] \\
\hline & $\mathrm{Pd}(\mathrm{II}) / \mathrm{GO} @ \mathrm{Fe}-\mathrm{FeO}$ & one-pot reaction & $10 \mathrm{~mol} \%$ & $60-90 \%$ & 6 runs & not calculated & [113] \\
\hline & $\mathrm{Pd} /$ Kaiser oxime resin & Mizoroki-Heck vinylation & $1.0 \mathrm{~mol} \%$ & $34-44 \%$ & $\begin{array}{c}\text { not } \\
\text { specified }\end{array}$ & not calculated & [137] \\
\hline & $\begin{array}{c}\mathrm{Cu}_{2}(\mathrm{OBA})_{2}(\mathrm{BPY})-\mathrm{MOF} \\
\mathrm{CuOMOFY} \\
\text { LS-FAS-Cu }\end{array}$ & $\begin{array}{l}\text { one-pot domino reaction } \\
\text { multi-component reaction } \\
\text { cyclocondensation }\end{array}$ & $\begin{array}{l}10 \mathrm{~mol} \% \\
0.7 \mathrm{~mol} \% \\
10 \mathrm{~mol} \%\end{array}$ & $\begin{array}{c}63-92 \% \\
91-94 \% \\
85 \%\end{array}$ & $\begin{array}{l}8 \text { runs } \\
\text { n.a. } \\
3 \text { runs }\end{array}$ & $\begin{array}{l}\text { not calculated } \\
\text { TOF }=67-104 \mathrm{~h}^{-1} \\
\text { not calculated }\end{array}$ & $\begin{array}{l}{[213]} \\
{[214]} \\
{[147]}\end{array}$ \\
\hline \multirow[t]{9}{*}{ quinoxaline } & $\mathrm{Au} / \mathrm{CeO}_{2}$ & oxidative coupling & 1.0 wt. $\%$ & $35-91 \%$ & 4 runs & $\begin{array}{c}\text { TOF }= \\
121 \mathrm{~h}^{-1}\end{array}$ & [89] \\
\hline & $\mathrm{Au} / \mathrm{CeO}_{2}$ & oxidative coupling & 1.0 wt. $\%$ & $24-80 \%$ & $\begin{array}{l}3 \text { runs } \\
4 \text { runs }+\end{array}$ & not calculated & [90] \\
\hline & $\mathrm{Au} / \mathrm{HT}$ & oxidative coupling & 0.7 wt. $\%$ & $80-85 \%$ & $\begin{array}{l}\text { calcina- } \\
\text { tion }\end{array}$ & $\mathrm{TOF}=132 \mathrm{~h}^{-1}$ & [89] \\
\hline & $\begin{array}{l}\mathrm{BiCl}_{3} \text {-perlite } \\
\mathrm{Zr}-\mathrm{CAP} \text {-SG }\end{array}$ & $\begin{array}{l}\text { condensation } \\
\text { condensation }\end{array}$ & $\begin{array}{l}5 \text { wt. } \% \\
10 \text { wt. } \%\end{array}$ & $\begin{array}{l}80-98 \% \\
83-95 \%\end{array}$ & $\begin{array}{l}5 \text { runs } \\
5 \text { runs }\end{array}$ & $\begin{array}{l}\text { not calculated } \\
\text { not calculated }\end{array}$ & $\begin{array}{l}{[111]} \\
{[166]}\end{array}$ \\
\hline & Cu(II)-DiAmSar/SBA-15 & condensation & $\begin{array}{l}0.008 \\
\mathrm{~mol} \%\end{array}$ & $88-99 \%$ & 7 runs & not calculated & [168] \\
\hline & Fe-Schiff base/SBA-15 & condensation & $0.14 \mathrm{~mol} \%$ & $98-99 \%$ & 6 runs & not calculated & [169] \\
\hline & $\mathrm{Zr}$ (IV)-Schiff base/SBA-15 & condensation & $0.2 \mathrm{~mol} \%$ & $98-99 \%$ & 7 runs & not calculated & [170] \\
\hline & Pd(II)-Schiff base/SBA-15 & condensation & $0.5 \mathrm{~mol} \%$ & $95-99 \%$ & 8 runs & not calculated & [171] \\
\hline & $\mathrm{ZrOL}_{2} @ S M N P$ & condensation & $\begin{array}{l}0.004 \\
\mathrm{~mol} \%\end{array}$ & $78-97 \%$ & 4 runs & not calculated & [207] \\
\hline \multirow[t]{3}{*}{ quinazoline } & $\mathrm{Fe}-\mathrm{Fe}_{3} \mathrm{C} / \mathrm{CN}-800$ & oxidative coupling & $4 \mathrm{~mol} \%$ & $78-98 \%$ & 6 runs & not calculated & [57] \\
\hline & $\mathrm{Ni}_{2} \mathrm{P} / N, P-\mathrm{C}-800$ & $\begin{array}{c}\text { oxidative cross- } \\
\text { dehydrogenative coupling }\end{array}$ & $7.5 \mathrm{~mol} \%$ & $71-94 \%$ & 5 runs & not calculated & [58] \\
\hline & $\begin{array}{c}\mathrm{Au} / \mathrm{TiO}_{2} \\
\mathrm{CuO} / \text { kaolin }\end{array}$ & $\begin{array}{l}\text { two hydrogen-transfer reaction } \\
\text { not specified }\end{array}$ & $\begin{array}{l}0.8 \mathrm{~mol} \% \\
3.4 \mathrm{~mol} \%\end{array}$ & $\begin{array}{l}31-99 \% \\
45-90 \%\end{array}$ & $\begin{array}{l}8 \text { runs } \\
4 \text { runs }\end{array}$ & $\begin{array}{l}\text { not calculated } \\
\text { not calculated }\end{array}$ & $\begin{array}{c}{[83]} \\
{[105]}\end{array}$ \\
\hline \multirow{6}{*}{$\begin{array}{c}\text { pyrido }[2,3-d] \\
\text { pyrimidine } \\
\text { pyrido }[2,3-b] \\
\text { pyrazine }\end{array}$} & $\mathrm{Cu}-\mathrm{TiO}_{2}$ & not specified & 1.0 wt. $\%$ & $58-94 \%$ & n.a. & not calculated & {$[86]$} \\
\hline & $\mathrm{Cu}(\mathrm{II})-\mathrm{DiAmSar} / \mathrm{SBA}-15$ & condensation & $\begin{array}{l}0.008 \\
\mathrm{~mol} \%\end{array}$ & $74-85 \%$ & 7 runs & not calculated & [168] \\
\hline & Fe-Schiff base/SBA-15 & condensation & $0.14 \mathrm{~mol} \%$ & $95-98 \%$ & 6 runs & not calculated & [169] \\
\hline & $\mathrm{Zr}$ (IV)-Schiff base/SBA-15 & condensation & $0.2 \mathrm{~mol} \%$ & $94-99 \%$ & 7 runs & not calculated & [170] \\
\hline & Pd(II)-Schiff base/SBA-15 & condensation & $0.5 \mathrm{~mol} \%$ & $95-98 \%$ & 8 runs & not calculated & [171] \\
\hline & $\mathrm{ZrOL}_{2} @ \mathrm{SMNP}$ & condensation & $\begin{array}{l}0.004 \\
\mathrm{~mol} \%\end{array}$ & $73-95 \%$ & 4 runs & not calculated & [207] \\
\hline \multirow{4}{*}{$\begin{array}{l}\text { 2,3-dihydro- } 1 H- \\
\text { benzazepine } \\
\text { 2,3-dihydro-1H-1,4- } \\
\text { benzodiazepine }\end{array}$} & Pd/Smopex ${ }^{\circledR}-234$ & $\begin{array}{l}\text { cyclocarbonylative } \\
\text { Sonogashira coupling }\end{array}$ & $0.4 \mathrm{~mol} \%$ & $63 \%$ & n.a. & not calculated & [125] \\
\hline & $\mathrm{Pt} / \mathrm{TiO}_{2}+\mathrm{MCM}-41$ & $\begin{array}{l}\text { hydrogenation/ } \\
\text { cyclocondensation } \\
\text { condensation/ }\end{array}$ & $1.0 \mathrm{wt} . \%$ & $84-95 \%$ & n.a. & $\mathrm{TOF}=25-111 \mathrm{~min}^{-1}$ & [87] \\
\hline & Cu@ß-CD@SPIONs & $\begin{array}{c}\text { cyclocondensation } /[3+2] \\
\text { cycloaddition }\end{array}$ & $1.0 \mathrm{~mol} \%$ & $71-87 \%$ & 5 runs & not calculated & [209] \\
\hline & $\mathrm{CuFe}_{2} \mathrm{O}_{4} @ \mathrm{MIL}-101(\mathrm{Cr})$ & cyclocondensation & $\begin{array}{l}0.005 \\
\mathrm{~mol} \%\end{array}$ & $87-95 \%$ & 6 runs & not calculated & [215] \\
\hline \multirow[t]{3}{*}{$\begin{array}{l}\text { 2,3-dihydro- } 1 H-1,5- \\
\text { benzodiazepine }\end{array}$} & $\mathrm{Zr} /$ Norit RX3Zr/xerogel & cyclocondensation & $100 \mathrm{mg}$ & up to $99 \%$ & n.a. & not calculated & [47] \\
\hline & $\mathrm{Fe}_{3} \mathrm{O}_{4} @ \mathrm{SiO}_{2}-\mathrm{CeCl}_{3}$ & $\begin{array}{l}\text { one-pot three-component } \\
\text { reaction }\end{array}$ & $\begin{array}{c}8 \\
\mathrm{mg} / \mathrm{mmol}\end{array}$ & $71-91 \%$ & 8 runs & not calculated & [116] \\
\hline & $\mathrm{FeCl}_{3} / \mathrm{PVP}$ & condensation/cyclization & $5.0 \mathrm{~mol} \%$ & $85-98 \%$ & 4 runs & not calculated & [119] \\
\hline
\end{tabular}

Author Contributions: Conceptualization, G.A.; data curation, L.A.A. and G.A.; writing-original draft preparation, L.A.A. and G.A.; writing-review and editing, G.A.; supervision, G.A. All authors have read and agreed to the published version of the manuscript.

Funding: This research was funded by the University of Pisa, grant number PRA_2020_21.

Conflicts of Interest: The authors declare no conflict of interest. 


\section{References}

1. Joule, J.A. Chapter Four-Natural Products Containing Nitrogen Heterocycles-Some Highlights 1990-2015. In Advances in Heterocyclic Chemistry; Scriven, E.F.V., Ramsden, C.A., Eds.; Academic Press: Cambridge, UK, 2016; pp. 81-106.

2. Prandi, C.; Occhiato, E.G. From synthetic control to natural products: A focus on N-heterocycles. Pest Manag. Sci. 2019, 75, 2385-2402. [CrossRef]

3. Kumar, D.; Kumar Jain, S. A Comprehensive Review of N-Heterocycles as Cytotoxic Agents. Curr. Med. Chem. 2016, 23, 4338-4394. [CrossRef] [PubMed]

4. Heravi, M.M.; Zadsirjan, V. Prescribed drugs containing nitrogen heterocycles: An overview. RSC Adv. 2020, 10, 44247-44311. [CrossRef]

5. Elgemeie, G.H.; Helal, M.H.; El-Sayed, H.M. Recent trends in synthesis and application of nitrogen heterocyclic azo dyes. Pigm. Resin Technol. 2001, 30, 210-228. [CrossRef]

6. Lipunova, G.N.; Nosova, E.V.; Charushin, V.N.; Chupakhin, O.N. Functionalized Quinazolines and Pyrimidines for Optoelectronic Materials. Curr. Org. Synth. 2018, 15, 793-814. [CrossRef]

7. Albano, G.; Aronica, L.A.; Minotto, A.; Cacialli, F.; Di Bari, L. Chiral Oligothiophenes with Remarkable Circularly Polarized Luminescence and Electroluminescence in Thin Films. Chem. Eur. J. 2020, 26, 16622-16627. [CrossRef] [PubMed]

8. Wang, Z.; Chen, B.; Zhao, J.; Zhang, Q.; Lin, Z.; Weng, J.; Huang, W. Recent progress in 1,4-diazafluorene-cored optoelectronic materials: A review. Dyes Pigm. 2021, 191, 109365. [CrossRef]

9. Albano, G.; Aronica, L.A. Potentiality and Synthesis of O- and N-Heterocycles: Pd-Catalyzed Cyclocarbonylative Sonogashira Coupling as a Valuable Route to Phthalans, Isochromans, and Isoindolines. Eur. J. Org. Chem. 2017, 2017, 7204-7221. [CrossRef]

10. Albano, G.; Aronica, L.A. Cyclization Reactions for the Synthesis of Phthalans and Isoindolines. Synthesis 2018, 50, 1209-1227.

11. Albano, G.; Aronica, L.A. From Alkynes to Heterocycles through Metal-Promoted Silylformylation and Silylcarbocyclization Reactions. Catalysts 2020, 10, 1012. [CrossRef]

12. Albano, G.; Aronica, L.A. Acyl Sonogashira Cross-Coupling: State of the Art and Application to the Synthesis of Heterocyclic Compounds. Catalysts 2020, 10, 25. [CrossRef]

13. Albano, G.; Morelli, M.; Aronica, L.A. Synthesis of Functionalised 3-Isochromanones by Silylcarbocyclisation/Desilylation Reactions. Eur. J. Org. Chem. 2017, 2017, 3473-3480. [CrossRef]

14. Aronica, L.A.; Albano, G.; Giannotti, L.; Meucci, E. Synthesis of N-Heteroaromatic Compounds through Cyclocarbonylative Sonogashira Reactions. Eur. J. Org. Chem. 2017, 2017, 955-963. [CrossRef]

15. Albano, G.; Morelli, M.; Lissia, M.; Aronica, L.A. Synthesis of Functionalised Indoline and Isoquinoline Derivatives through a Silylcarbocyclisation/Desilylation Sequence. ChemistrySelect 2019, 4, 2505-2511. [CrossRef]

16. Albano, G.; Giuntini, S.; Aronica, L.A. Synthesis of 3-Alkylideneisoindolin-1-ones via Sonogashira Cyclocarbonylative Reactions of 2-Ethynylbenzamides. J. Org. Chem. 2020, 85, 10022-10034. [CrossRef]

17. Anastas, P.; Eghbali, N. Green Chemistry: Principles and Practice. Chem. Soc. Rev. 2010, 39, 301-312. [CrossRef]

18. Schlögl, R. Heterogeneous Catalysis. Angew. Chem. Int. Ed. 2015, 54, 3465-3520. [CrossRef]

19. Friend, C.M.; Xu, B. Heterogeneous Catalysis: A Central Science for a Sustainable Future. Acc. Chem. Res. 2017, 50, 517-521. [CrossRef]

20. Astruc, D.; Lu, F.; Aranzaes, J.R. Nanoparticles as Recyclable Catalysts: The Frontier between Homogeneous and Heterogeneous Catalysis. Angew. Chem. Int. Ed. 2005, 44, 7852-7872. [CrossRef]

21. Heravi, M.M.; Alishiri, T. Application of nanomaterials in heterocyclic chemistry. Heterocycles 2012, 85, 545-586. [CrossRef]

22. Elwahy, A.H.M.; Shaaban, M.R. Synthesis of heterocycles and fused heterocycles catalyzed by nanomaterials. RSC Adv. 2015, 5, 75659-75710. [CrossRef]

23. Banerjee, B. Recent developments on nano-ZnO catalyzed synthesis of bioactive heterocycles. J. Nanostruct. Chem. 2017, 7 , 389-413. [CrossRef]

24. Nasrollahzadeh, M. Advances in Magnetic Nanoparticles-Supported Palladium Complexes for Coupling Reactions. Molecules 2018, 23, 2532. [CrossRef]

25. Kaur, G.; Devi, P.; Thakur, S.; Kumar, A.; Chandel, R.; Banerjee, B. Magnetically Separable Transition Metal Ferrites: Versatile Heterogeneous Nano-Catalysts for the Synthesis of Diverse Bioactive Heterocycles. ChemistrySelect 2019, 4, 2181-2199. [CrossRef]

26. Aqeel Ashraf, M.; Liu, Z.; Yang, Y.; Zhang, D. Magnetic nanoparticles supported copper catalysts: Synthesis of heterocyclic scaffolds. Synth. Commun. 2020, 50, 2885-2905. [CrossRef]

27. Huang, W.; Cheng, Q.; Ma, D. Recent reports on magnetic nanoparticles supported metallic catalysts: Synthesis of heterocycles. Synth. Commun. 2021, 51, 1321-1339. [CrossRef]

28. Elwahy, A.H.M.; Shaaban, M.R. Synthesis of heterocycles catalyzed by iron oxide nanoparticles. Heterocycles 2017, 94, 595-655. [CrossRef]

29. Bhaskaruni, S.V.H.S.; Maddila, S.; Gangu, K.K.; Jonnalagadda, S.B. A review on multi-component green synthesis of N-containing heterocycles using mixed oxides as heterogeneous catalysts. Arab. J. Chem. 2020, 13, 1142-1178. [CrossRef]

30. Sharma, A.; Gudala, S.; Ambati, S.R.; Penta, S.; Mahapatra, S.P.; Vedula, R.R.; Pola, S.; Acharya, B. Synthesis of Heterocyclic Compounds Catalyzed by Metal/Metal Oxide-Multiwall Carbon Nanotube Nanocomposites. J. Chin. Chem. Soc. 2017, 64, 589-606. [CrossRef] 
31. Rai, A.; Ranganath, K.V.S. Recyclable catalysts for the synthesis of heterocyclic compounds using carbon materials. J. Heterocycl. Chem. 2021, 58, 1039-1057. [CrossRef]

32. Daştan, A.; Kulkarni, A.; Török, B. Environmentally benign synthesis of heterocyclic compounds by combined microwave-assisted heterogeneous catalytic approaches. Green Chem. 2012, 14, 17-37. [CrossRef]

33. Mandoli, A. Recent Advances in Recoverable Systems for the Copper-Catalyzed Azide-Alkyne Cycloaddition Reaction (CuAAC). Molecules 2016, 21, 1174. [CrossRef]

34. McAteer, C.H.; Murugan, R.; Subba Rao, Y.V. Chapter Six-Heterogeneously Catalyzed Synthesis of Heterocyclic Compounds. In Advances in Heterocyclic Chemistry; Scriven, E.F.V., Ramsden, C.A., Eds.; Academic Press: Cambridge, UK, 2017 ; pp. 173-205.

35. Truong, C.C.; Ngo, H.L. Sustainable synthesis of nitrogen heterocycles from carbon dioxide and aromatic amines over heterogeneous catalysts. J. $\mathrm{CO}_{2}$ Util. 2020, 42, 101325. [CrossRef]

36. Fraile, J.M.; García, J.I.; Mayoral, J.A.; Pires, E. Heterogenization on Inorganic Supports: Methods and Applications. In Heterogenized Homogeneous Catalysts for Fine Chemicals Production: Materials and Processes; Barbaro, P., Liguori, F., Eds.; Springer: Dordrecht, The Netherlands, 2010; pp. 65-121.

37. Gruber, M.; Chouzier, S.; Koehler, K.; Djakovitch, L. Palladium on activated carbon: A valuable heterogeneous catalyst for one-pot multi-step synthesis. Appl. Catal. A 2004, 265, 161-169. [CrossRef]

38. Landge, S.M.; Schmidt, A.; Outerbridge, V.; Török, B. Synthesis of Pyrazoles by a One-Pot Tandem Cyclization-Dehydrogenation Approach on Pd/C/K-10 Catalyst. Synlett 2007, 2007, 1600-1604. [CrossRef]

39. Weires, N.A.; Boster, J.; Magolan, J. Combined Pd/C and Montmorillonite Catalysis for One-Pot Synthesis of Benzimidazoles. Eur. J. Org. Chem. 2012, 2012, 6508-6512. [CrossRef] [PubMed]

40. Rossy, C.; Majimel, J.; Delapierre, M.T.; Fouquet, E.; Felpin, F.-X. Palladium and copper-supported on charcoal: A heterogeneous multi-task catalyst for sequential Sonogashira-Click and Click-Heck reactions. J. Organomet. Chem. 2014, 755, 78-85. [CrossRef]

41. Lipshutz, B.H.; Taft, B.R. Heterogeneous Copper-in-Charcoal-Catalyzed Click Chemistry. Angew. Chem. Int. Ed. 2006, 45, 8235-8238. [CrossRef]

42. Lee, C.-T.; Huang, S.; Lipshutz, B.H. Copper-in-Charcoal-Catalyzed, Tandem One-Pot Diazo Transfer-Click Reactions. Adv. Synth Catal. 2009, 351, 3139-3142. [CrossRef]

43. Alonso, F.; Moglie, Y.; Radivoy, G.; Yus, M. Multicomponent Synthesis of 1,2,3-Triazoles in Water Catalyzed by Copper Nanoparticles on Activated Carbon. Adv. Synth. Catal. 2010, 352, 3208-3214. [CrossRef]

44. Alonso, F.; Moglie, Y.; Radivoy, G.; Yus, M. Click chemistry from organic halides, diazonium salts and anilines in water catalysed by copper nanoparticles on activated carbon. Org. Biomol. Chem. 2011, 9, 6385-6395. [CrossRef] [PubMed]

45. Albaladejo, M.J.; Alonso, F.; Yus, M. Synthesis of Indolizines and Heterocyclic Chalcones Catalyzed by Supported Copper Nanoparticles. Chem. Eur. J. 2013, 19, 5242-5245. [CrossRef] [PubMed]

46. Siddiki, S.M.A.H.; Touchy, A.S.; Chaudhari, C.; Kon, K.; Toyao, T.; Shimizu, K.-i. Synthesis of 2,5-disubstituted pyrroles via dehydrogenative condensation of secondary alcohols and 1,2-amino alcohols by supported platinum catalysts. Org. Chem. Front. 2016, 3, 846-851. [CrossRef]

47. Godino-Ojer, M.; Milla-Diez, L.; Matos, I.; Durán-Valle, C.J.; Bernardo, M.; Fonseca, I.M.; Pérez Mayoral, E. Enhanced Catalytic Properties of Carbon supported Zirconia and Sulfated Zirconia for the Green Synthesis of Benzodiazepines. ChemCatChem 2018, 10, 5215-5223. [CrossRef]

48. Shaygan Nia, A.; Rana, S.; Döhler, D.; Noirfalise, X.; Belfiore, A.; Binder, W.H. Click chemistry promoted by graphene supported copper nanomaterials. Chem. Commun. 2014, 50, 15374-15377. [CrossRef]

49. Hussain, N.; Gogoi, P.; Das, M.R.; Sengupta, P.; Fedorov, V.E.; Asanov, I.P.; Kozlova, M.N.; Artemkina, S.B. Development of novel efficient 2D nanocomposite catalyst towards the three-component coupling reaction for the synthesis of imidazo [1,2-a] pyridines. Appl. Catal. A 2017, 542, 368-379. [CrossRef]

50. Demirci, T.; Çelik, B.; Yıldız, Y.; Eriş, S.; Arslan, M.; Sen, F.; Kilbas, B. One-pot synthesis of Hantzsch dihydropyridines using a highly efficient and stable PdRuNi@GO catalyst. RSC Adv. 2016, 6, 76948-76956. [CrossRef]

51. Santra, S.; Ranjan, P.; Bera, P.; Ghosh, P.; Mandal, S.K. Anchored palladium nanoparticles onto single walled carbon nanotubes: Efficient recyclable catalyst for N-containing heterocycles. RSC Adv. 2012, 2, 7523-7533. [CrossRef]

52. Godino-Ojer, M.; López-Peinado, A.J.; Maldonado-Hódar, F.J.; Bailón-García, E.; Pérez-Mayoral, E. Cobalt oxide-carbon nanocatalysts with highly enhanced catalytic performance for the green synthesis of nitrogen heterocycles through the Friedländer condensation. Dalton Trans. 2019, 48,5637-5648. [CrossRef]

53. Ezhilmathi, P.; Thirumalai, K.; Swaminathan, M.; Krishnasamy, K. One-Pot Synthesis of Tetra Substituted Imidazoles Catalyzed by Fly Ash Supported Bi2O3-ZnO. J. Nanosci. Nanotechnol. 2019, 19, 8163-8171. [CrossRef]

54. Kazemi Movahed, S.; Salari, P.; Kasmaei, M.; Armaghan, M.; Dabiri, M.; Amini, M.M. Copper nanoparticles incorporated on a mesoporous carbon nitride, an excellent catalyst in the Huisgen 1,3-dipolar cycloaddition and N-arylation of N-heterocycles. Appl. Organomet. Chem. 2018, 32, e3914. [CrossRef]

55. Phatake, V.V.; Bhanage, B.M. Cu@U-g- $\mathrm{C}_{3} \mathrm{~N}_{4}$ Catalyzed Cyclization of o-Phenylenediamines for the Synthesis of Benzimidazoles by Using $\mathrm{CO}_{2}$ and Dimethylamine Borane as a Hydrogen Source. Catal. Lett. 2019, 149, 347-359. [CrossRef]

56. Gong, Z.; Lei, Y.; Zhou, P.; Zhang, Z. One-pot synthesis of N-substituted pyrroles from nitro compounds and 2,5-hexadione over a heterogeneous cobalt catalyst. New J. Chem. 2017, 41, 10613-10618. [CrossRef] 
57. Ma, Z.; Song, T.; Yuan, Y.; Yang, Y. Synergistic catalysis on Fe-Nx sites and Fe nanoparticles for efficient synthesis of quinolines and quinazolinones via oxidative coupling of amines and aldehydes. Chem. Sci. 2019, 10, 10283-10289. [CrossRef]

58. Song, T.; Ren, P.; Ma, Z.; Xiao, J.; Yang, Y. Highly Dispersed Single-Phase $\mathrm{Ni}_{2} \mathrm{P}$ Nanoparticles on N,P-Codoped Porous Carbon for Efficient Synthesis of N-Heterocycles. ACS Sustain. Chem. Eng. 2020, 8, 267-277. [CrossRef]

59. Díaz-Ortiz, A.; de la Hoz, A.; Moreno, A.; Sánchez-Migallón, A.; Valiente, G. Synthesis of 1,3,5-triazines in solvent-free conditions catalysed by silica-supported lewis acids. Green Chem. 2002, 4, 339-343. [CrossRef]

60. Soltani Rad, M.N.; Behrouz, S.; Doroodmand, M.M.; Movahediyan, A. Copper-doped silica cuprous sulfate (CDSCS) as a highly efficient and new heterogeneous nano catalyst for [3+2] Huisgen cycloaddition. Tetrahedron 2012, 68, 7812-7821. [CrossRef]

61. Inamdar, S.M.; More, V.K.; Mandal, S.K. CuO nano-particles supported on silica, a new catalyst for facile synthesis of benzimidazoles, benzothiazoles and benzoxazoles. Tetrahedron Lett. 2013, 54, 579-583. [CrossRef]

62. Maleki, A. One-pot multicomponent synthesis of diazepine derivatives using terminal alkynes in the presence of silica-supported superparamagnetic iron oxide nanoparticles. Tetrahedron Lett. 2013, 54, 2055-2059. [CrossRef]

63. Arabpourian, K.; Behbahani, F.K. Synthesis of Pyrrole Derivatives Promoted by $\mathrm{Fe}\left(\mathrm{ClO}_{4}\right)_{3} / \mathrm{SiO}_{2}$ as an Environmentally Friendly Catalyst. Russ. J. Org. Chem. 2019, 55, 682-685. [CrossRef]

64. Safari, J.; Gandomi-Ravandi, S.; Naseh, S. Efficient, green and solvent-free synthesis of tetrasubstituted imidazoles using $\mathrm{SbCl}_{3} / \mathrm{SiO}_{2}$ as heterogeneous catalyst. J. Chem. Sci. 2013, 125, 827-833. [CrossRef]

65. Safari, J.; Naseh, S.; Zarnegar, Z.; Akbari, Z. Applications of microwave technology to rapid synthesis of substituted imidazoles on silica-supported $\mathrm{SbCl}_{3}$ as an efficient heterogeneous catalyst. J. Taibah Univ. Sci. 2014, 8, 323-330. [CrossRef]

66. Atar, A.B.; Kim, J.S.; Lim, K.T.; Jeong, Y.T. Bridging homogeneous and heterogeneous catalysis with $\mathrm{CAN}_{\mathrm{SiO}}$ as a solid catalyst for four-component reactions for the synthesis of tetrasubstituted pyrroles. New J. Chem. 2015, 39, 396-402. [CrossRef]

67. Maddila, S.; Valand, J.; Bandaru, H.; Yalagala, K.; Lavanya, P. Ag Loaded on $\mathrm{SiO}_{2}$ as an Efficient and Recyclable Heterogeneous Catalyst for the Synthesis of Chloro-8-substituted-9H-purines. J. Heterocycl. Chem. 2016, 53, 319-324. [CrossRef]

68. So, M.-H.; Liu, Y.; Ho, C.-M.; Lam, K.-Y.; Che, C.-M. Silica-Supported Gold Nanoparticles Catalyzed One-Pot, Tandem Aerobic Oxidative Cyclization Reaction for Nitrogen-Containing Polyheterocyclic Compounds. ChemCatChem 2011, 3, 386-393. [CrossRef]

69. Abdollahi-Alibeik, M.; Rezaeipoor-Anari, A. BF3/MCM-41 as nano structured solid acid catalyst for the synthesis of 3-iminoarylimidazo [1,2-a] pyridines. Catal. Sci. Technol. 2014, 4, 1151-1159. [CrossRef]

70. Samanta, P.K.; Banerjee, R.; Richards, R.M.; Biswas, P. Mesoporous silica supported ytterbium as catalyst for synthesis of 1,2-disubstituted benzimidazoles and 2-substituted benzimidazoles. Appl. Organomet. Chem. 2018, 32, e4507. [CrossRef]

71. Samanta, P.K.; Biswas, R.; Das, T.; Nandi, M.; Adhikary, B.; Richards, R.M.; Biswas, P. Mesoporous silica supported samarium as recyclable heterogeneous catalyst for synthesis of 5-substituted tetrazole and 2-substituted benzothiazole. J. Porous Mater. 2019, 26, 145-155. [CrossRef]

72. Naeimi, H.; Nejadshafiee, V. Efficient one-pot click synthesis of $\beta$-hydroxy-1,2,3-triazoles catalyzed by copper(i)@phosphorated $\mathrm{SiO}_{2}$ via multicomponent reaction in aqueous media. New J. Chem. 2014, 38, 5429-5435. [CrossRef]

73. Kumar, A.; Koul, S.; Razdan, T.K.; Kapoor, K.K. A new and convenient one-pot solid supported synthesis of 2,4,6-triarylpyridines. Tetrahedron Lett. 2006, 47, 837-842. [CrossRef]

74. Mukherjee, N.; Ahammed, S.; Bhadra, S.; Ranu, B.C. Solvent-free one-pot synthesis of 1,2,3-triazole derivatives by the 'Click' reaction of alkyl halides or aryl boronic acids, sodium azide and terminal alkynes over a $\mathrm{Cu} / \mathrm{Al}_{2} \mathrm{O}_{3}$ surface under ball-milling. Green Chem. 2013, 15, 389-397. [CrossRef]

75. Agalave, S.G.; Pharande, S.G.; Gade, S.M.; Pore, V.S. Alumina-Supported Copper Iodide: An Efficient and Recyclable Catalyst for Microwave-Assisted Synthesis of 1,4-Disubstituted 1,2,3-Triazoles via Three-Component Reaction in Water. Asian J. Org. Chem. 2015, 4, 943-951. [CrossRef]

76. Maddila, S.; Maddila, S.N.; Jonnalagadda, S.B.; Lavanya, P. Reusable Ce-V Loaded Alumina Catalyst for Multicomponent Synthesis of Substituted Pyridines in Green Media. J. Heterocycl. Chem. 2016, 53, 658-664. [CrossRef]

77. Park, J.W.; Song, Y.-H. Synthesis of thienopyrimidine-pyrazolo [3,4-b] pyridine hybrids. Heterocycl. Commun. 2017, 23, 281-285. [CrossRef]

78. Zsolnai, D.; Mayer, P.; Szőri, K.; London, G. Pd/ $\mathrm{Al}_{2} \mathrm{O}_{3}$-catalysed redox isomerisation of allyl alcohol: Application in aldol condensation and oxidative heterocyclization reactions. Catal. Sci. Technol. 2016, 6, 3814-3820. [CrossRef]

79. Mazarío, J.; Raad, Z.; Concepción, P.; Cerdá-Moreno, C.; Domine, M.E. Pd supported on mixed metal oxide as an efficient catalyst for the reductive amination of bio-derived acetol to 2-methylpiperazine. Catal. Sci. Technol. 2020, 10, 8049-8063. [CrossRef]

80. Bellezza, D.; Zaragozá, R.J.; José Aurell, M.; Ballesteros, R.; Ballesteros-Garrido, R. Acceptorless dehydrogenative condensation: Synthesis of indoles and quinolines from diols and anilines. Org. Biomol. Chem. 2021, 19, 677-683. [CrossRef]

81. Hao, L.; Zhao, Y.; Yu, B.; Zhang, H.; Xu, H.; Liu, Z. Au catalyzed synthesis of benzimidazoles from 2-nitroanilines and $\mathrm{CO}_{2} / \mathrm{H}_{2}$. Green Chem. 2014, 16, 3039-3044. [CrossRef]

82. Tang, L.; Guo, X.; Yang, Y.; Zha, Z.; Wang, Z. Gold nanoparticles supported on titanium dioxide: An efficient catalyst for highly selective synthesis of benzoxazoles and benzimidazoles. Chem. Commun. 2014, 50, 6145-6148. [CrossRef]

83. Tang, L.; Yang, Y.; Wen, L.; Zhang, S.; Zha, Z.; Wang, Z. Supported gold-catalyzed and ammonia-promoted selective synthesis of quinazolines in aqueous media. Org. Chem. Front. 2015, 2, 114-118. [CrossRef]

84. Tzani, M.A.; Gabriel, C.; Lykakis, I.N. Selective Synthesis of Benzimidazoles from o-Phenylenediamine and Aldehydes Promoted by Supported Gold Nanoparticles. Nanomaterials 2020, 10, 2405. [CrossRef] [PubMed] 
85. Meng, X.; Wang, Y.; Yu, C.; Zhao, P. Heterogeneously copper-catalyzed oxidative synthesis of imidazo [1,2-a] pyridines using 2-aminopyridines and ketones under ligand-and additive-free conditions. RSC Adv. 2014, 4, 27301-27307. [CrossRef]

86. Geesi, M.H.; Ouerghi, O.; Elsanousi, A.; Kaiba, A.; Riadi, Y. Ultrasound-Assisted Preparation of Cu-Doped $\mathrm{TiO}_{2} \mathrm{Nanoparticles} \mathrm{as}^{-}$ a Nanocatalyst for Sonochemical Synthesis of Pyridopyrimidines. Polycycl. Aromat. Compd. 2020, 42, 80-90. [CrossRef]

87. Climent, M.J.; Corma, A.; Iborra, S.; Santos, L.L. Multisite Solid Catalyst for Cascade Reactions: The Direct Synthesis of Benzodiazepines from Nitro Compounds. Chem. Eur. J. 2009, 15, 8834-8841. [CrossRef]

88. Ruiz, V.R.; Corma, A.; Sabater, M.J. New route for the synthesis of benzimidazoles by a one-pot multistep process with mono and bifunctional solid catalysts. Tetrahedron 2010, 66, 730-735. [CrossRef]

89. Climent, M.J.; Corma, A.; Hernández, J.C.; Hungría, A.B.; Iborra, S.; Martínez-Silvestre, S. Biomass into chemicals: One-pot twoand three-step synthesis of quinoxalines from biomass-derived glycols and 1,2-dinitrobenzene derivatives using supported gold nanoparticles as catalysts. J. Catal. 2012, 292, 118-129. [CrossRef]

90. Climent, M.J.; Corma, A.; Iborra, S.; Martínez-Silvestre, S. Gold Catalysis Opens Up a New Route for the Synthesis of Benzimidazoylquinoxaline Derivatives from Biomass-Derived Products (Glycerol). ChemCatChem 2013, 5, 3866-3874. [CrossRef]

91. Shimura, S.; Miura, H.; Wada, K.; Hosokawa, S.; Yamazoe, S.; Inoue, M. Ceria-supported ruthenium catalysts for the synthesis of indole via dehydrogenative N-heterocyclization. Catal. Sci. Technol. 2011, 1, 1340-1346. [CrossRef]

92. Maddila, S.; Rana, S.; Pagadala, R.; Kankala, S.; Maddila, S.; Jonnalagadda, S.B. Synthesis of pyrazole-4-carbonitrile derivatives in aqueous media with $\mathrm{CuO} / \mathrm{ZrO}_{2}$ as recyclable catalyst. Catal. Commun. 2015, 61, 26-30. [CrossRef]

93. Shabalala, S.; Maddila, S.; van Zyl, W.E.; Jonnalagadda, S.B. A facile, efficacious and reusable $\mathrm{Sm}_{2} \mathrm{O}_{3} / \mathrm{ZrO}_{2}$ catalyst for the novel synthesis of functionalized 1,4-dihydropyridine derivatives. Catal. Commun. 2016, 79, 21-25. [CrossRef]

94. Ahmadian, H.; Veisi, H.; Karami, C.; Sedrpoushan, A.; Nouri, M.; Jamshidi, F.; Alavioon, I. Cobalt manganese oxide nanoparticles as recyclable catalyst for efficient synthesis of 2-aryl-1-arylmethyl-1H-1,3-benzimidazoles under solvent-free conditions. Appl. Organomet. Chem. 2015, 29, 266-269. [CrossRef]

95. Meng, X.; Zhang, J.; Chen, B.; Jing, Z.; Zhao, P. Copper supported on H+-modified manganese oxide octahedral molecular sieves $(\mathrm{Cu} / \mathrm{H}-\mathrm{OMS}-2)$ as a heterogeneous biomimetic catalyst for the synthesis of imidazo [1,2-a]-N-heterocycles. Catal. Sci. Technol. 2016, 6, 890-896. [CrossRef]

96. Albadi, J.; Alihosseinzadeh, A.; Mansournezhad, A. Regioselective synthesis of 1,2,3-triazoles catalyzed over ZnO supported copper oxide nanocatalyst as a new and efficient recyclable catalyst in water. Acta Chim. Slov. 2015, 62, 8. [CrossRef] [PubMed]

97. Moromi, S.K.; Touchy, A.S.; Hakim Siddiki, S.M.A.; Ali, M.A.; Shimizu, K.-i. Synthesis of indoles via dehydrogenative N-heterocyclization by supported platinum catalysts. RSC Adv. 2015, 5, 1059-1062. [CrossRef]

98. Anil Kumar, B.S.P.; Harsha Vardhan Reddy, K.; Madhav, B.; Ramesh, K.; Nageswar, Y.V.D. Magnetically separable CuFe ${ }_{2} \mathrm{O}_{4}$ nano particles catalyzed multicomponent synthesis of 1,4-disubstituted 1,2,3-triazoles in tap water using 'click chemistry'. Tetrahedron Lett. 2012, 53, 4595-4599. [CrossRef]

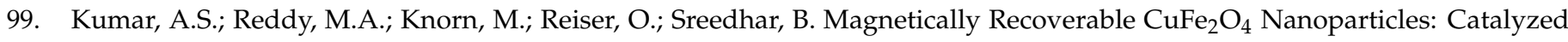
Synthesis of Aryl Azides and 1,4-Diaryl-1,2,3-triazoles from Boronic Acids in Water. Eur. J. Org. Chem. 2013, 2013, 4674-4680. [CrossRef]

100. El-Remaily, M.A.E.A.A.A.; Abu-Dief, A.M. $\mathrm{CuFe}_{2} \mathrm{O}_{4}$ nanoparticles: An efficient heterogeneous magnetically separable catalyst for synthesis of some novel propynyl-1H-imidazoles derivatives. Tetrahedron 2015, 71, 2579-2584. [CrossRef]

101. Sanasi, P.D.; Santhipriya, D.; Ramesh, Y.; Kumar, M.R.; Swathi, B.; Rao, K.J. Nano copper and cobalt ferrites as heterogeneous catalysts for the one-pot synthesis of 2,4,5-tri substituted imidazoles. J. Chem. Sci. 2014, 126, 1715-1720. [CrossRef]

102. Yamane, Y.; Liu, X.; Hamasaki, A.; Ishida, T.; Haruta, M.; Yokoyama, T.; Tokunaga, M. One-Pot Synthesis of Indoles and Aniline Derivatives from Nitroarenes under Hydrogenation Condition with Supported Gold Nanoparticles. Org. Lett. 2009, 11, 5162-5165. [CrossRef]

103. Moghaddam, F.M.; Koushki Foroushani, B.; Rezvani, H.R. Nickel ferrite nanoparticles: An efficient and reusable nanocatalyst for a neat, one-pot and four-component synthesis of pyrroles. RSC Adv. 2015, 5, 18092-18096. [CrossRef]

104. Ravikumar Naik, T.R.; Shivashankar, S.A. Heterogeneous bimetallic $\mathrm{ZnFe}_{2} \mathrm{O}_{4}$ nanopowder catalyzed synthesis of Hantzsch 1,4-dihydropyridines in water. Tetrahedron Lett. 2016, 57, 4046-4049. [CrossRef]

105. Zhang, J.; Yu, C.; Wang, S.; Wan, C.; Wang, Z. A novel and efficient methodology for the construction of quinazolines based on supported copper oxide nanoparticles. Chem. Commun. 2010, 46, 5244-5246. [CrossRef] [PubMed]

106. Mohammed, S.; Padala, A.K.; Dar, B.A.; Singh, B.; Sreedhar, B.; Vishwakarma, R.A.; Bharate, S.B. Recyclable clay supported Cu (II) catalyzed tandem one-pot synthesis of 1-aryl-1,2,3-triazoles. Tetrahedron 2012, 68, 8156-8162. [CrossRef]

107. Dubey, N.; Sharma, P.; Kumar, A. Clay-Supported Cu (II) Catalyst: An Efficient, Heterogeneous, and Recyclable Catalyst for Synthesis of 1,4-Disubstituted 1,2,3-Triazoles from Alloxan-Derived Terminal Alkyne and Substituted Azides Using Click Chemistry. Synth. Commun. 2015, 45, 2608-2626. [CrossRef]

108. Qiao, B.; Zhang, L.; Li, R. A highly active and recyclable catalyst for the synthesis of indole and phenyl ether. RSC Adv. 2015, 5, 93463-93469. [CrossRef]

109. Pagadala, R.; Maddila, S.; Dasireddy, V.D.B.C.; Jonnalagadda, S.B. Zn-VCO3 hydrotalcite: A highly efficient and reusable heterogeneous catalyst for the Hantzsch dihydropyridine reaction. Catal. Commun. 2014, 45, 148-152. [CrossRef]

110. Yum, E.K.; Hong, K.B. Synthesis of pyrrolo-heterocycles via Pd-loaded zeolite catalyzed annulation of o-haloaromatic amine with terminal alkynes. Tetrahedron 2017, 73, 6581-6586. [CrossRef] 
111. Brindha, K.; Amutha, P.; Krishnakumar, B.; do Nascimento Sobral, A.J.F. BiCl3-modified perlite as an effective catalyst for selective organic transformations: A green protocol. Res. Chem. Intermed. 2019, 45, 4367-4381. [CrossRef]

112. Xiong, X.; Chen, H.; Tang, Z.; Jiang, Y. Supported $\mathrm{CuBr}$ on graphene oxide $/ \mathrm{Fe}_{3} \mathrm{O}_{4}$ : A highly efficient, magnetically separable catalyst for the multi-gram scale synthesis of 1,2,3-triazoles. RSC Adv. 2014, 4, 9830-9837. [CrossRef]

113. Verma, S.; Verma, D.; Jain, S.L. Magnetically separable palladium-graphene nanocomposite as heterogeneous catalyst for the synthesis of 2-alkylquinolines via one pot reaction of anilines with alkenyl ethers. Tetrahedron Lett. 2014, 55, 2406-2409. [CrossRef]

114. Zhang, M.; Lu, J.; Zhang, J.-N.; Zhang, Z.-H. Magnetic carbon nanotube supported $\mathrm{Cu}\left(\mathrm{CoFe}_{2} \mathrm{O}_{4} / \mathrm{CNT}-\mathrm{Cu}\right)$ catalyst: A sustainable catalyst for the synthesis of 3-nitro-2-arylimidazo [1,2-a] pyridines. Catal. Commun. 2016, 78, 26-32. [CrossRef]

115. Prakash, P.; Kumar, R.A.; Miserque, F.; Geertsen, V.; Gravel, E.; Doris, E. Carbon nanotube-copper ferrite-catalyzed aqueous 1,3-dipolar cycloaddition of in situ-generated organic azides with alkynes. Chem. Commun. 2018, 54, 3644-3647. [CrossRef]

116. Zhou, L.; Wang, M.; Wang, K.; Wen, T.; Wang, L. $\mathrm{Fe}_{3} \mathrm{O}_{4} @ \mathrm{SiO}_{2}-\mathrm{CeCl}_{3}$ Catalyzed Chemoselective Synthesis of Functionalized 3-Substituted-1,5-Benzodiazepines via One-pot Multicomponent and Domino Reactions. Appl. Organomet. Chem. 2020, 34 , e5707. [CrossRef]

117. Hajizadeh, Z.; Radinekiyan, F.; Eivazzadeh-keihan, R.; Maleki, A. Development of novel and green $\mathrm{NiFe}_{2} \mathrm{O}_{4} /$ geopolymer nanocatalyst based on bentonite for synthesis of imidazole heterocycles by ultrasonic irradiations. Sci. Rep. 2020, $10,11671$. [CrossRef] [PubMed]

118. Bergbreiter, D.E. Organic Polymers as a Catalyst Recovery Vehicle. In Chiral Catalyst Immobilization and Recycling; De Vos, D.E., Vankelecom, I.F.J., Jacobs, P.A., Eds.; Wiley-VCH: Weinheim, Germany, 2000; pp. 43-80.

119. Adharvana Chari, M.; Syamasundar, K. Polymer (PVP) supported ferric chloride: An efficient and recyclable heterogeneous catalyst for high yield synthesis of 1,5-benzodiazepine derivatives under solvent free conditions and microwave irradiation. Catal. Commun. 2005, 6, 67-70. [CrossRef]

120. Hashemi, E.; Beheshtiha, Y.S.; Ahmadi, S.; Heravi, M.M. In situ prepared CuI nanoparticles on modified poly (styrene-co-maleic anhydride): An efficient and recyclable catalyst for the azide-alkyne click reaction in water. Transit. Met. Chem. 2014, 39, 593-601. [CrossRef]

121. Reddy, C.B.; Bharti, R.; Kumar, S.; Das, P. Supported palladium nanoparticles-catalyzed decarboxylative coupling approaches to aryl alkynes, indoles and pyrrolines synthesis. RSC Adv. 2016, 6, 71117-71121. [CrossRef]

122. Abdollahi-Alibeik, M.; Moosavifard, M. FeCl -Doped Polyaniline Nanoparticles as Reusable Heterogeneous Catalyst for the Synthesis of 2-Substituted Benzimidazoles. Synth. Commun. 2010, 40, 2686-2695. [CrossRef]

123. Chopra, R.; Kumar, M.; Neelam, N.; Bhalla, V. Visible light promoted PANI@Au: CuO catalyzed sequential amination, azidation and annulation for the preparation of 2-arylbenzimidazoles. Green Chem. 2019, 21, 3666-3674. [CrossRef]

124. Santra, S.; Dhara, K.; Ranjan, P.; Bera, P.; Dash, J.; Mandal, S.K. A supported palladium nanocatalyst for copper free acyl Sonogashira reactions: One-pot multicomponent synthesis of N-containing heterocycles. Green Chem. 2011, 13, $3238-3247$. [CrossRef]

125. Albano, G.; Evangelisti, C.; Aronica, L.A. Palladium Nanoparticles Supported on Smopex-234 ${ }^{\circledR}$ as Valuable Catalysts for the Synthesis of Heterocycles. Catalysts 2021, 11, 706. [CrossRef]

126. Albano, G.; Evangelisti, C.; Aronica, L.A. Hydrogenolysis of Benzyl Protected Phenols and Aniline Promoted by Supported Palladium Nanoparticles. ChemistrySelect 2017, 2, 384-388. [CrossRef]

127. Albano, G.; Interlandi, S.; Evangelisti, C.; Aronica, L.A. Polyvinylpyridine-Supported Palladium Nanoparticles: A Valuable Catalyst for the Synthesis of Alkynyl Ketones via Acyl Sonogashira Reactions. Catal. Lett. 2020, 150, 652-659. [CrossRef]

128. Rad, M.N.S.; Behrouz, S.; Movahedian, A.; Doroodmand, M.M.; Ghasemi, Y.; Rasoul-Amini, S.; Gandomani, A.-R.A.; Rezaie, R Doped Nano-Sized Copper (I) Oxide $\left(\mathrm{Cu}_{2} \mathrm{O}\right)$ on Melamine-Formaldehyde Resin: A Highly Efficient Heterogeneous Nano Catalyst for 'Click' Synthesis of Some Novel 1H-1,2,3-Triazole Derivatives Having Antibacterial Activity. Helv. Chim. Acta 2013, 96, 688-701. [CrossRef]

129. Elavarasan, S.; Bhaumik, A.; Sasidharan, M. An Efficient Mesoporous Cu-Organic Nanorod for Friedländer Synthesis of Quinoline and Click Reactions. ChemCatChem 2019, 11, 4340-4350. [CrossRef]

130. Khatun, R.; Biswas, S.; Biswas, I.H.; Riyajuddin, S.; Haque, N.; Ghosh, K.; Islam, S.M. Cu-NPs@COF: A potential heterogeneous catalyst for $\mathrm{CO}_{2}$ fixation to produce 2-oxazolidinones as well as benzimidazoles under moderate reaction conditions. J. CO $\mathrm{Ctil}$. 2020, 40, 101180. [CrossRef]

131. Grigg, R.; York, M. Bimetallic catalytic cascade ring closing metathesis-intramolecular Heck reactions using a fluorous biphasic solvent system or a polymer-supported palladium catalyst. Tetrahedron Lett. 2000, 41, 7255-7258. [CrossRef]

132. Bakherad, M.; Bahramian, B.; Nasr-Isfahani, H.; Keivanloo, A.; Doostmohammadi, N. Polystyrene-supported palladium (II) ethylenediamine complex: A highly active and recyclable catalyst for the synthesis of 2-benzylimidazo [2,1-b] pyridines through heteroannulation of acetylenic compounds. J. Heterocycl. Chem. 2009, 46, 100-104. [CrossRef]

133. Molla, R.A.; Roy, A.S.; Ghosh, K.; Salam, N.; Iqubal, M.A.; Tuhina, K.; Islam, S.M. Polymer anchored ruthenium complex: A highly active and recyclable catalyst for one-pot azide-alkyne cycloaddition and transfer-hydrogenation of ketones under mild conditions. J. Organomet. Chem. 2015, 776, 170-179. [CrossRef]

134. Chtchigrovsky, M.; Primo, A.; Gonzalez, P.; Molvinger, K.; Robitzer, M.; Quignard, F.; Taran, F. Functionalized Chitosan as a Green, Recyclable, Biopolymer-Supported Catalyst for the [3+2] Huisgen Cycloaddition. Angew. Chem. Int. Ed. 2009, 48, 5916-5920. [CrossRef] 
135. Chan, T.R.; Fokin, V.V. Polymer-Supported Copper (I) Catalysts for the Experimentally Simplified Azide-Alkyne Cycloaddition. QSAR Comb. Sci. 2007, 26, 1274-1279. [CrossRef]

136. Pucci, A.; Albano, G.; Pollastrini, M.; Lucci, A.; Colalillo, M.; Oliva, F.; Evangelisti, C.; Marelli, M.; Santalucia, D.; Mandoli, A. Supported Tris-Triazole Ligands for Batch and Continuous-Flow Copper-Catalyzed Huisgen 1,3-Dipolar Cycloaddition Reactions. Catalysts 2020, 10, 434. [CrossRef]

137. Alacid, E.; Nájera, C. Arylation of Allyl Alcohols in Organic and Aqueous Media Catalyzed by Oxime-Derived Palladacycles: Synthesis of $\beta$-Arylated Carbonyl Compounds. Adv. Synth. Catal. 2007, 349, 2572-2584. [CrossRef]

138. Biswas, I.H.; Biswas, S.; Islam, M.S.; Riyajuddin, S.; Sarkar, P.; Ghosh, K.; Islam, S.M. Catalytic synthesis of benzimidazoles and organic carbamates using a polymer supported zinc catalyst through $\mathrm{CO}_{2}$ fixation. New J. Chem. 2019, 43, 14643-14652. [CrossRef]

139. Kröll, R.M.; Schuler, N.; Lubbad, S.; Buchmeiser, M.R. A ROMP-derived, polymer-supported chiral Schrock catalyst for enantioselective ring-closing olefin metathesis. Chem. Commun. 2003, 21, 2742-2743. [CrossRef] [PubMed]

140. Hlil, A.R.; Moncho, S.; Tuba, R.; Elsaid, K.; Szarka, G.; Brothers, E.N.; Grubbs, R.H.; Al-Hashimi, M.; Bazzi, H.S. Synthesis and catalytic activity of supported acenaphthoimidazolylidene N-heterocyclic carbene ruthenium complex for ring closing metathesis (RCM) and ring opening metathesis polymerization (ROMP). J. Catal. 2016, 344, 100-107. [CrossRef]

141. Chtchigrovsky, M.; Lin, Y.; Ouchaou, K.; Chaumontet, M.; Robitzer, M.; Quignard, F.; Taran, F. Dramatic Effect of the Gelling Cation on the Catalytic Performances of Alginate-Supported Palladium Nanoparticles for the Suzuki-Miyaura Reaction. Chem. Mater. 2012, 24, 1505-1510. [CrossRef]

142. Xiang, Z.; Chen, Y.; Liu, Q.; Lu, F. A highly recyclable dip-catalyst produced from palladium nanoparticle-embedded bacterial cellulose and plant fibers. Green Chem. 2018, 20, 1085-1094. [CrossRef]

143. Zhu, Y.; Li, Z.; Chen, J. Applications of lignin-derived catalysts for green synthesis. Green Energy Environ. 2019, 4, $210-244$. [CrossRef]

144. Rizzo, G.; Albano, G.; Lo Presti, M.; Milella, A.; Omenetto, F.G.; Farinola, G.M. Palladium Supported on Silk Fibroin for Suzuki-Miyaura Cross-Coupling Reactions. Eur. J. Org. Chem. 2020, 2020, 6992-6996. [CrossRef]

145. Khalafi-Nezhad, A.; Mohammadi, S. Highly efficient synthesis of 1- and 5-substituted 1H-tetrazoles using chitosan derived magnetic ionic liquid as a recyclable biopolymer-supported catalyst. RSC Adv. 2013, 3, 4362-4371. [CrossRef]

146. Baig, R.B.N.; Varma, R.S. Copper on chitosan: A recyclable heterogeneous catalyst for azide-alkyne cycloaddition reactions in water. Green Chem. 2013, 15, 1839-1843. [CrossRef]

147. Lai, B.; Ye, M.; Liu, P.; Li, M.; Bai, R.; Gu, Y. A novel and robust heterogeneous Cu catalyst using modified lignosulfonate as support for the synthesis of nitrogen-containing heterocycles. Beilstein J. Org. Chem. 2020, 16, 2888-2902. [CrossRef] [PubMed]

148. Sharghi, H.; Ebrahimpourmoghaddam, S.; Doroodmand, M.M.; Purkhosrow, A. Synthesis of Vasorelaxaing 1,4-Disubstituted 1,2,3-Triazoles Catalyzed by a $4^{\prime}$-Phenyl-2,2' $: 6^{\prime}, 2^{\prime \prime}$-Terpyridine Copper (II) Complex Immobilized on Activated Multiwalled Carbon Nanotubes. Asian J. Org. Chem. 2012, 1, 377-388. [CrossRef]

149. Sharghi, H.; Ebrahimpourmoghaddam, S.; Doroodmand, M.M. Facile synthesis of 5-substituted-1H-tetrazoles and 1-substituted1 H-tetrazoles catalyzed by recyclable $4^{\prime}$-phenyl-2, $2^{\prime}: 6^{\prime}, 2^{\prime \prime}$-terpyridine copper (II) complex immobilized onto activated multi-walled carbon nanotubes. J. Organomet. Chem. 2013, 738, 41-48. [CrossRef]

150. Liu, G.; Wu, B.; Zhang, J.; Wang, X.; Shao, M.; Wang, J. Controlled Reversible Immobilization of Ru Carbene on Single-Walled Carbon Nanotubes: A New Strategy for Green Catalytic Systems Based on a Solvent Effect on $\pi-\pi$ Interaction. Inorg. Chem. 2009, 48, 2383-2390. [CrossRef] [PubMed]

151. Nasrallah, H.; Germain, S.; Queval, P.; Bouvier, C.; Mauduit, M.; Crévisy, C.; Schulz, E. Non covalent immobilization of pyrenetagged ruthenium complexes onto graphene surfaces for recycling in olefin metathesis reactions. J. Mol. Catal. A Chem. 2016, 425, 136-146. [CrossRef]

152. Shaygan Nia, A.; Rana, S.; Döhler, D.; Jirsa, F.; Meister, A.; Guadagno, L.; Koslowski, E.; Bron, M.; Binder, W.H. Carbon-Supported Copper Nanomaterials: Recyclable Catalysts for Huisgen [3+2] Cycloaddition Reactions. Chem. Eur. J. 2015, 21, 10763-10770. [CrossRef]

153. Collinson, J.-M.; Wilton-Ely, J.D.E.T.; Díez-González, S. Reusable and highly active supported copper (i)-NHC catalysts for Click chemistry. Chem. Commun. 2013, 49, 11358-11360. [CrossRef]

154. Sharghi, H.; Shiri, P.; Aberi, M. Five-membered N-Heterocycles Synthesis Catalyzed by Nano-silica Supported Copper (II)-2imino-1,2-diphenylethan-1-ol Complex. Catal. Lett. 2017, 147, 2844-2862. [CrossRef]

155. Sharghi, H.; Aberi, M.; Shiri, P. Supported benzimidazole-salen Cu (II) complex: An efficient, versatile and highly reusable nanocatalyst for one-pot synthesis of hybrid molecules. Appl. Organomet. Chem. 2018, 32, e4446. [CrossRef]

156. Sharghi, H.; Aberi, M.; Shiri, P. Silica-supported Cu (II)-quinoline complex: Efficient and recyclable nanocatalyst for one-pot synthesis of benzimidazolquinoline derivatives and 2H-indazoles. Appl. Organomet. Chem. 2019, 33, e4974. [CrossRef]

157. Veerakumar, P.; Velayudham, M.; Lu, K.-L.; Rajagopal, S. Highly dispersed silica-supported nanocopper as an efficient heterogeneous catalyst: Application in the synthesis of 1,2,3-triazoles and thioethers. Catal. Sci. Technol. 2011, 1, 1512-1525. [CrossRef]

158. Nasr-Esfahani, M.; Mohammadpoor-Baltork, I.; Khosropour, A.R.; Moghadam, M.; Mirkhani, V.; Tangestaninejad, S. Synthesis and characterization of $\mathrm{Cu}$ (II) containing nanosilica triazine dendrimer: A recyclable nanocomposite material for the synthesis of benzimidazoles, benzothiazoles, bis-benzimidazoles and bis-benzothiazoles. J. Mol. Catal. A Chem. 2013, 379, 243-254. [CrossRef] 
159. Jumde, R.P.; Evangelisti, C.; Mandoli, A.; Scotti, N.; Psaro, R. Aminopropyl-silica-supported Cu nanoparticles: An efficient catalyst for continuous-flow Huisgen azide-alkyne cycloaddition (CuAAC). J. Catal. 2015, 324, 25-31. [CrossRef]

160. Sarmiento, J.T.; Suárez-Pantiga, S.; Olmos, A.; Varea, T.; Asensio, G. Silica-Immobilized NHC-Gold (I) Complexes: Versatile Catalysts for the Functionalization of Alkynes under Batch and Continuous Flow Conditions. ACS Catal. 2017, 7, 7146-7155. [CrossRef]

161. Tyrrell, E.; Whiteman, L.; Williams, N. Sonogashira Cross-Coupling Reactions and Construction of the Indole Ring System Using a Robust, Silica-Supported Palladium Catalyst. Synthesis 2009, 2009, 829-835. [CrossRef]

162. Abarghooei, M.A.; Mohebat, R.; Karimi-Jaberi, Z.; Mosslemin, M.H. Nano-silica supported palladium nanoparticles: A sustainable nanocatalyst for efficient synthesis of 2,3-diarylimidazo [1,2-a] pyridines at low catalyst loading. Catal. Commun. 2018, 105, 59-64. [CrossRef]

163. Michalek, F.; Bannwarth, W. Application of a Grubbs-Hoveyda Metathesis Catalyst Noncovalently Immobilized by FluorousFluorous Interactions. Helv. Chim. Acta 2006, 89, 1030-1037. [CrossRef]

164. Allen, D.P.; Van Wingerden, M.M.; Grubbs, R.H. Well-Defined Silica-Supported Olefin Metathesis Catalysts. Org. Lett. 2009, 11, 1261-1264. [CrossRef]

165. Monge-Marcet, A.; Pleixats, R.; Cattoën, X.; Wong Chi Man, M. Sol-gel immobilized Hoveyda-Grubbs complex through the NHC ligand: A recyclable metathesis catalyst. J. Mol. Catal. A Chem. 2012, 357, 59-66. [CrossRef]

166. Sharma, R.K.; Sharma, C. Zirconium (IV)-modified silica gel: Preparation, characterization and catalytic activity in the synthesis of some biologically important molecules. Catal. Commun. 2011, 12, 327-331. [CrossRef]

167. Hajjami, M.; Ghorbani, F.; Yousofvand, Z. Copper (I) complex of 1,3-DimethylBarbituric acid modified SBA-15 and its catalytic role for the synthesis of 2,3-Dihydroquinazolin-4(1H)-ones and Imidazoles. Appl. Organomet. Chem. 2017, 31, e3843. [CrossRef]

168. Mohammadi, M.; Bardajee, G.R.; Pesyan, N.N. Efficient solvent-free synthesis of pyridopyrazine and quinoxaline derivatives using copper-DiAmSar complex anchored on SBA-15 as a reusable catalyst. Chin. J. Catal. 2015, 36, 1379-1386. [CrossRef]

169. Malakooti, R.; Bardajee, G.R.; Hadizadeh, S.; Atashin, H.; Khanjari, H. An iron Schiff base complex loaded mesoporous silica nanoreactor as a catalyst for the synthesis of pyrazine-based heterocycles. Transit. Met. Chem. 2014, 39, 47-54. [CrossRef]

170. Malakooti, R.; Bardajee, G.R.; Mahmoudi, H.; Kakavand, N. Zirconium Schiff-Base Complex Modified Mesoporous Silica as an Efficient Catalyst for the Synthesis of Nitrogen Containing Pyrazine Based Heterocycles. Catal. Lett. 2013, 143, 853-861. [CrossRef]

171. Bardajee, G.R.; Malakooti, R.; Abtin, I.; Atashin, H. Palladium Schiff-base complex loaded SBA-15 as a novel nanocatalyst for the synthesis of 2,3-disubstituted quinoxalines and pyridopyrazine derivatives. Microporous Mesoporous Mater. 2013, 169, 67-74. [CrossRef]

172. Batail, N.; Bendjeriou, A.; Djakovitch, L.; Dufaud, V. Larock indole synthesis using palladium complexes immobilized onto mesoporous silica. Appl. Catal. A 2010, 388, 179-187. [CrossRef]

173. Skowerski, K.; Pastva, J.; Czarnocki, S.J.; Janoscova, J. Exceptionally Stable and Efficient Solid Supported Hoveyda-Type Catalyst. Org. Process Res. Dev. 2015, 19, 872-877. [CrossRef]

174. Nie, Q.; Yao, F.; Yi, F.; Cai, M. A heterogeneous gold (I)-catalyzed cascade annulation of aldehydes with propargylamine leading to 3-substituted 2,5-dimethylpyrazines. J. Organomet. Chem. 2017, 846, 343-350. [CrossRef]

175. Wei, L.; Yao, F.; Yi, F.; Cai, M. Heterogeneous Gold (I)-Catalysed Annulation between 2-Aminopyridines and Propiolaldehydes Leading to 3-Acylimidazo [1,2-A] Pyridines. J. Chem. Res. 2018, 42, 341-346. [CrossRef]

176. Jiang, G.; Lin, Y.; Cai, M.; Zhao, H. Recyclable Heterogeneous Copper (II)-Catalyzed Oxidative Cyclization of 2-Pyridine Ketone Hydrazones Towards [1,2,3] Triazolo [1,5-a] pyridines. Synthesis 2019, 51, 4487-4497. [CrossRef]

177. Garcés, K.; Fernández-Alvarez, F.J.; García-Orduña, P.; Lahoz, F.J.; Pérez-Torrente, J.J.; Oro, L.A. Grafting of Copper (I)-NHC Species on MCM-41: Homogeneous versus Heterogeneous Catalysis. ChemCatChem 2015, 7, 2501-2507. [CrossRef]

178. Nikoorazm, M.; Tahmasbi, B.; Gholami, S.; Moradi, P. Copper and nickel immobilized on cytosine@MCM-41: As highly efficient, reusable and organic-inorganic hybrid nanocatalysts for the homoselective synthesis of tetrazoles and pyranopyrazoles. Appl. Organomet. Chem. 2020, 34, e5919. [CrossRef]

179. Deiana, L.; Afewerki, S.; Palo-Nieto, C.; Verho, O.; Johnston, E.V.; Córdova, A. Highly Enantioselective Cascade Transformations by Merging Heterogeneous Transition Metal Catalysis with Asymmetric Aminocatalysis. Sci. Rep. 2012, 2, 851. [CrossRef]

180. Deiana, L.; Jiang, Y.; Palo-Nieto, C.; Afewerki, S.; Incerti-Pradillos, C.A.; Verho, O.; Tai, C.-W.; Johnston, E.V.; Córdova, A. Combined Heterogeneous Metal/Chiral Amine: Multiple Relay Catalysis for Versatile Eco-Friendly Synthesis. Angew. Chem. Int. Ed. 2014, 53, 3447-3451. [CrossRef]

181. Lu, J.; Ma, E.-Q.; Liu, Y.-H.; Li, Y.-M.; Mo, L.-P.; Zhang, Z.-H. One-pot three-component synthesis of 1,2,3-triazoles using magnetic NiFe2O4-glutamate-Cu as an efficient heterogeneous catalyst in water. RSC Adv. 2015, 5, 59167-59185. [CrossRef]

182. Norouzi, M.; Ghorbani-Choghamarani, A.; Nikoorazm, M. Heterogeneous Cu (ii)/1-His@Fe3O4 nanocatalyst: A novel, efficient and magnetically-recoverable catalyst for organic transformations in green solvents. RSC Adv. 2016, 6, 92387-92401. [CrossRef]

183. Habibi, D.; Pakravan, N.; Arabi, A.; Kaboudvand, Z. Preparation of $\mathrm{Fe}_{3} \mathrm{O}_{4} @ 5,10$-dihydropyrido [2,3-b] quinoxaline-7,8-diol copper complex: A capable nanocatalyst for the green synthesis of 1-substituted $1 \mathrm{H}$-tetrazoles. Appl. Organomet. Chem. 2018, 32, e3988. [CrossRef]

184. Kumari, M.; Jain, Y.; Yadav, P.; Laddha, H.; Gupta, R. Synthesis of $\mathrm{Fe}_{3} \mathrm{O}_{4}$-DOPA-Cu Magnetically Separable Nanocatalyst: A Versatile and Robust Catalyst for an Array of Sustainable Multicomponent Reactions under Microwave Irradiation. Catal. Lett. 2019, 149, 2180-2194. [CrossRef] 
185. Darabi, M.; Tamoradi, T.; Ghadermazi, M.; Ghorbani-Choghamarani, A. A magnetically retrievable heterogeneous copper nanocatalyst for the synthesis of 5-substituted tetrazoles and oxidation reactions. Transit. Met. Chem. 2017, 42, 703-710. [CrossRef]

186. Jafari, F.; Ghorbani-Choghamarani, A.; Hasanzadeh, N. Guanidine complex of copper supported on boehmite nanoparticles as practical, recyclable, chemo and homoselective organic-inorganic hybrid nanocatalyst for organic reactions. Appl. Organomet. Chem. 2020, 34, e5901. [CrossRef]

187. Dehghani, F.; Sardarian, A.R.; Esmaeilpour, M. Salen complex of $\mathrm{Cu}$ (II) supported on superparamagnetic $\mathrm{Fe}_{3} \mathrm{O}_{4} @ \mathrm{SiO}_{2}$ nanoparticles: An efficient and recyclable catalyst for synthesis of 1- and 5-substituted 1H-tetrazoles. J. Organomet. Chem. 2013, 743, 87-96. [CrossRef]

188. Xiong, X.; Cai, L. Application of magnetic nanoparticle-supported CuBr: A highly efficient and reusable catalyst for the one-pot and scale-up synthesis of 1,2,3-triazoles under microwave-assisted conditions. Catal. Sci. Technol. 2013, 3, 1301-1307. [CrossRef]

189. Bhardwaj, M.; Jamwal, B.; Paul, S. Novel Cu (0)- $\mathrm{Fe}_{3} \mathrm{O}_{4} @ \mathrm{SiO}_{2} / \mathrm{NH}_{2}$ cel as an Efficient and Sustainable Magnetic Catalyst for the Synthesis of 1,4-Disubstituted-1,2,3-triazoles and 2-Substituted-Benzothiazoles via One-Pot Strategy in Aqueous Media. Catal. Lett. 2016, 146, 629-644. [CrossRef]

190. Esmaeilpour, M.; Sardarian, A.R.; Firouzabadi, H. Dendrimer-encapsulated Cu (II) nanoparticles immobilized on superparamagnetic $\mathrm{Fe}_{3} \mathrm{O}_{4} @ \mathrm{SiO}_{2}$ nanoparticles as a novel recyclable catalyst for $\mathrm{N}$-arylation of nitrogen heterocycles and green synthesis of 5-substituted 1H-tetrazoles. Appl. Organomet. Chem. 2018, 32, e4300. [CrossRef]

191. Wang, D.; Etienne, L.; Echeverria, M.; Moya, S.; Astruc, D. A Highly Active and Magnetically Recoverable Tris (triazolyl)-CuI Catalyst for Alkyne-Azide Cycloaddition Reactions. Chem. Eur. J. 2014, 20, 4047-4054. [CrossRef]

192. Moghaddam, F.M.; Ayati, S.E. Copper immobilized onto a triazole functionalized magnetic nanoparticle: A robust magnetically recoverable catalyst for "click" reactions. RSC Adv. 2015, 5, 3894-3902. [CrossRef]

193. Moghaddam, F.M.; Ayati, S.E.; Firouzi, H.R.; Ghorbani, F. Immobilization of copper ions onto $\alpha$-amidotriazole-functionalized magnetic nanoparticles and their application in the synthesis of triazole derivatives in water. Appl. Organomet. Chem. 2016, 30, 488-493. [CrossRef]

194. Ariannezhad, M.; Habibi, D.; Heydari, S. Copper nanoparticles: A capable and versatile catalyst for the synthesis of diverse 1-phenyl-1H-tetrazoles from amino acids. Polyhedron 2019, 160, 170-179. [CrossRef]

195. Vibhute, S.P.; Mhaldar, P.M.; Korade, S.N.; Gaikwad, D.S.; Shejawal, R.V.; Pore, D.M. Synthesis of magnetically separable catalyst $\mathrm{Cu}-\mathrm{ACP}-\mathrm{Am}-\mathrm{Fe}_{3} \mathrm{O}_{4} @ \mathrm{SiO}_{2}$ for Huisgen 1,3-dipolar cycloaddition. Tetrahedron Lett. 2018, 59, 3643-3652. [CrossRef]

196. Tajbakhsh, M.; Farhang, M.; Hosseinzadeh, R.; Sarrafi, Y. Nano $\mathrm{Fe}_{3} \mathrm{O}_{4}$ supported biimidazole Cu (i) complex as a retrievable catalyst for the synthesis of imidazo [1,2-a] pyridines in aqueous medium. RSC Adv. 2014, 4, 23116-23124. [CrossRef]

197. Tajbakhsh, M.; Farhang, M.; Baghbanian, S.M.; Hosseinzadeh, R.; Tajbakhsh, M. Nano magnetite supported metal ions as robust, efficient and recyclable catalysts for green synthesis of propargylamines and 1,4-disubstituted 1,2,3-triazoles in water. New J. Chem. 2015, 39, 1827-1839. [CrossRef]

198. Esmaeilpour, M.; Javidi, J.; Zahmatkesh, S. One-pot synthesis of 1- and 5-substituted 1H-tetrazoles using 1,4-dihydroxyanthraquinonecopper (II) supported on superparamagnetic $\mathrm{Fe}_{3} \mathrm{O}_{4} @ \mathrm{SiO}_{2}$ magnetic porous nanospheres as a recyclable catalyst. Appl. Organomet. Chem. 2016, 30, 897-904. [CrossRef]

199. Zahmatkesh, S.; Esmaeilpour, M.; Javidi, J. 1,4-Dihydroxyanthraquinone-copper (ii) supported on superparamagnetic $\mathrm{Fe}_{3} \mathrm{O}_{4} @ \mathrm{SiO}_{2}$ : An efficient catalyst for $\mathrm{N}$-arylation of nitrogen heterocycles and alkylamines with aryl halides and click synthesis of 1-aryl-1,2,3-triazole derivatives. RSC Adv. 2016, 6, 90154-90164. [CrossRef]

200. Sardarian, A.R.; Eslahi, H.; Esmaeilpour, M. Copper (II) Complex Supported on $\mathrm{Fe}_{3} \mathrm{O}_{4} @ \mathrm{SiO}_{2}$ Coated by Polyvinyl Alcohol as Reusable Nanocatalyst in N-Arylation of Amines and N (H)-Heterocycles and Green Synthesis of 1H-Tetrazoles. ChemistrySelect 2018, 3, 1499-1511. [CrossRef]

201. Jahanshahi, R.; Akhlaghinia, B. CuII immobilized on guanidinated epibromohydrin functionalized $\gamma-\mathrm{Fe}_{2} \mathrm{O}_{3} @ \mathrm{TiO}_{2}\left(\gamma-\mathrm{Fe}_{2} \mathrm{O}_{3} @ \mathrm{TiO}_{2}-\right.$ EG-CuII): A novel magnetically recyclable heterogeneous nanocatalyst for the green one-pot synthesis of 1,4-disubstituted 1,2,3-triazoles through alkyne-azide cycloaddition in water. RSC Adv. 2016, 6, 29210-29219.

202. Chen, S.-W.; Zhang, Z.-C.; Zhai, N.-N.; Zhong, C.-M.; Lee, S.-G. The effect of silica-coating on catalyst recyclability in ionic magnetic nanoparticle-supported Grubbs-Hoveyda catalysts for ring-closing metathesis. Tetrahedron 2015, 71, 648-653. [CrossRef]

203. Rakhtshah, J.; Salehzadeh, S.; Gowdini, E.; Maleki, F.; Baghery, S.; Zolfigol, M.A. Synthesis of pyrazole derivatives in the presence of a dioxomolybdenum complex supported on silica-coated magnetite nanoparticles as an efficient and easily recyclable catalyst. RSC Adv. 2016, 6, 104875-104885. [CrossRef]

204. Li, B.-L.; Zhang, M.; Hu, H.-C.; Du, X.; Zhang, Z.-H. Nano-CoFe $\mathrm{O}_{4}$ supported molybdenum as an efficient and magnetically recoverable catalyst for a one-pot, four-component synthesis of functionalized pyrroles. New J. Chem. 2014, 38, $2435-2442$. [CrossRef]

205. Li, B.-L.; Hu, H.-C.; Mo, L.-P.; Zhang, Z.-H. Nano $\mathrm{CoFe}_{2} \mathrm{O}_{4}$ supported antimony (iii) as an efficient and recyclable catalyst for one-pot three-component synthesis of multisubstituted pyrroles. RSC Adv. 2014, 4, 12929-12943. [CrossRef]

206. Ma, F.-P.; Li, P.-H.; Li, B.-L.; Mo, L.-P.; Liu, N.; Kang, H.-J.; Liu, Y.-N.; Zhang, Z.-H. A recyclable magnetic nanoparticles supported antimony catalyst for the synthesis of N-substituted pyrroles in water. Appl. Catal. A 2013, 457, 34-41. [CrossRef]

207. Jafarpour, M.; Rezaeifard, A. A zirconium Schiff base complex immobilized on starch-coated maghemite nanoparticles catalyzes heterogeneous condensation of 1,2-diamines with 1,2-dicarbonyl compounds. Transit. Met. Chem. 2016, 41, 205-211. [CrossRef] 
208. Maleki, A.; Movahed, H.; Paydar, R. Design and development of a novel cellulose $/ \gamma-\mathrm{Fe}_{2} \mathrm{O}_{3} /$ Ag nanocomposite: A potential green catalyst and antibacterial agent. RSC Adv. 2016, 6, 13657-13665. [CrossRef]

209. Mahdavi, M.; Lijan, H.; Bahadorikhalili, S.; Ma'mani, L.; Rashidi Ranjbar, P.; Shafiee, A. Copper supported $\beta$-cyclodextrin grafted magnetic nanoparticles as an efficient recyclable catalyst for one-pot synthesis of 1-benzyl-1H-1,2,3-triazoldibenzodiazepinone derivatives via click reaction. RSC Adv. 2016, 6, 28838-28843. [CrossRef]

210. Chetia, M.; Ali, A.A.; Bhuyan, D.; Saikia, L.; Sarma, D. Magnetically recoverable chitosan-stabilised copper-iron oxide nanocomposite material as an efficient heterogeneous catalyst for azide-alkyne cycloaddition reactions. New J. Chem. 2015, 39, $5902-5907$. [CrossRef]

211. Zarnegar, Z.; Safari, J. Catalytic activity of $\mathrm{Cu}$ nanoparticles supported on $\mathrm{Fe}_{3} \mathrm{O}_{4}$-polyethylene glycol nanocomposites for the synthesis of substituted imidazoles. New J. Chem. 2014, 38, 4555-4565. [CrossRef]

212. Zohreh, N.; Hosseini, S.H.; Pourjavadi, A.; Bennett, C. Immobilized copper (II) on nitrogen-rich polymer-entrapped Fe $\mathrm{O}_{4}$ nanoparticles: A highly loaded and magnetically recoverable catalyst for aqueous click chemistry. Appl. Organomet. Chem. 2016, 30, 73-80. [CrossRef]

213. Dang, H.V.; Le, H.T.B.; Tran, L.T.B.; Ha, H.Q.; Le, H.V.; Phan, N.T.S. Copper-catalyzed one-pot domino reactions via C-H bond activation: Synthesis of 3-aroylquinolines from 2-aminobenzylalcohols and propiophenones under metal-organic framework catalysis. RSC Adv. 2018, 8, 31455-31464. [CrossRef]

214. Martín, N.; Dusselier, M.; De Vos, D.E.; Cirujano, F.G. Metal-Organic Framework Derived Metal Oxide Clusters in Porous Aluminosilicates: A Catalyst Design for the Synthesis of Bioactive aza-Heterocycles. ACS Catal. 2019, 9, 44-48. [CrossRef]

215. Gupta, A.; Sarkar, F.K.; Sarkar, R.; Jamatia, R.; Lee, C.Y.; Gupta, G.; Pal, A.K. Development of a new catalytic and sustainable methodology for the synthesis of benzodiazepine triazole scaffold using magnetically separable CuFe $\mathrm{O}_{4} @ \mathrm{MIL}-101$ (Cr) nanocatalyst in aqueous medium. Appl. Organomet. Chem. 2020, 34, e5782. [CrossRef] 